

\section{INFORMATION TO USERS}

This manuscript has been reproduced from the microfilm master. UMI films the text directly from the original or copy submitted. Thus, some thesis and dissertation copies are in typewriter face, while others may be from any type of computer printer.

The quality of this reproduction is dependent upon the quality of the copy submitted. Broken or indistinct print, colored or poor quality illustrations and photographs, print bleedthrough, substandard margins, and improper alignment can adversely affect reproduction.

In the unlikely, event that the author did not send UMI a complete manuscript and there are missing pages, these will be noted. Also, if unauthorized copyright material had to be removed, a note will indicate the deletion.

Oversize materials (e.g., maps, drawings, charts) are reproduced by sectioning the original, beginning at the upper left-hand corner and continuing from left to right in equal sections with small overlaps. Each original is also photographed in one exposure and is included in reduced form at the back of the book.

Photographs included in the original manuscript have been reproduced

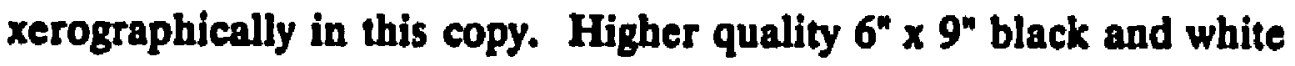
photographic prints are available for any photographs or illustrations appearing in this copy for an additional charge. Contact UMI directly to order.

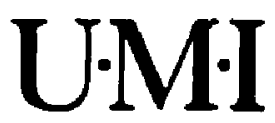

Universily Microfilms Internatıonal

A Bell \& Howell Iniormation Company

300 North Zeeb Road. Ann Arbor. MI 48106.1346 USA

$313 / 761-4700 \quad 800 / 521-0600$ 


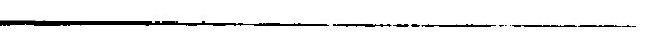


Order Number 9420950

A parallel binary atructured LMS algorithm for transveraal adaptive filters

Eshghi, Mohammad, Ph.D.

The Ohio State University, 1994 


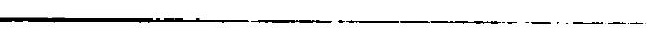




\title{
A PARAllel Binary StRUCtUREd LMS AlgorithM FOR TRANSVERSAL ADAPTIVE FILTERS
}

\author{
DISSERTATION
}

\author{
Presented in Partial Fulfillment of the Requirements for \\ the Degree Doctor of Philosophy in the Graduate \\ School of The Ohio State University
}

By

Mohammad Eshghi, $* * * * *$

The Ohio State University

1994

Dissertation Committee:

Approved by

Prof. Joanne DeGroat

Prof. Fusun Ozguner

Prof. Ashok Krishnamurthy

Prof. Steven Bibyk

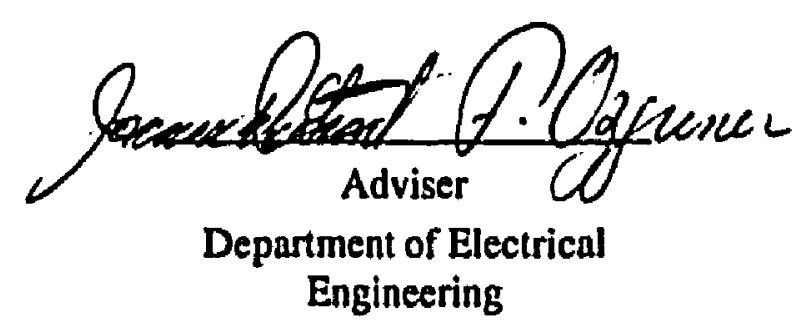


Dedicated To My Parents,

ALI AKBAR \& MALIHE

\section{ESHGHI,}

who taught me the most valuable lessons of the life,

LOVE and ADORATION 


\section{ACKNOWLEDGEMENTS}

I would like to express my decpest gratitude to Prof. Joan DeGroat, my thesis advisor, for her guidance throughout this research. I would like to express my appreciation to Prof. Fusun Ozguner, my academic advisor, for her help and encouragement throughout my Ph.D. program. I also wish to thank the rest of my dissertation committec, Prof. Steven Bibyk and Prof. Ashok Krishnamurthy, for their contribution to this research and critical review of the dissertation.

My deepest thanks are extended to all my friends, in Iran, in Europe, and in the USA, for their companionship and support.

My heartiest appreciation and acknowledgment goes to my parents, Ali Akbar and Malihe, my sisters, Fateme, Sedighe, Susan, Talihe, and Nahid, my brothers, Ahmad, Ali, Hossein, and reza, and my entire family for their encouragement, devotion, love, and endless support of my academic pursuits, without which this accomplishment could not have been possible. 


\begin{abstract}
- VITA
October 31, 1954 Born in Shahroud, Iran

September 1978 Bachelor of Science in Electrical Enginecring, Sharif University Of Technology, Tehran, Iran.

March 1988 Master of Science in Electrical Engineering, Ohio University, Athens, Ohio.
\end{abstract}

\title{
Publications
}

J. A. Starzyk and M. Eshghi, "Highly Parallel Adaptive Filter," Intl. Symp. on Circuits and Systems, Portland, Oregon, May 1989.

\section{Fields of Study}

\author{
Major Field: Electrical Engineering \\ Fields of Interest: Computer Engineering \\ parallel Processing \\ Signal Processing \\ VLSI/ VHDL design
}




\section{TABLE OF CONTENTS}

ACKNOWLEDGEMENTS $\ldots \ldots \ldots \ldots \ldots \ldots \ldots \ldots$ iii

viTA $\ldots \ldots \ldots \ldots \ldots \ldots \ldots \ldots \ldots \ldots \ldots \ldots \ldots$ iv

LIST OF FIGURES $\ldots \ldots \ldots \ldots \ldots \ldots \ldots \ldots \ldots$ viii

LIST OF TABLES $\ldots \ldots \ldots \ldots \ldots \ldots \ldots \ldots \ldots \ldots \ldots \ldots \ldots$

CHAPTER PAGE

I Introduction $\ldots \ldots \ldots \ldots \ldots \ldots \ldots \ldots \ldots \ldots \ldots \ldots$

II Adaptive Filters $\ldots \ldots \ldots \ldots \ldots \ldots \ldots \ldots$

2.1 Definition of Adaptive Fitters . . . . . . . . . . . . 5

2.2 Some Applications For Digital Adaptive Filters . . . . . . . 7

2.2.1 Unknown system identification . . . . . . . . . . 7

2.2.2 Noise cancellation in the data transmission line . . . . . . 9

2.2.3 Signal Prediction . . . . . . . . . . . . 11

2.3 Performance Index Minimization . . . . . . . . . . . . 12

2.4 Adaptive Filter Algorithms . . . . . . . . . . . . 13

2.4 .1 Wiener . . . . . . . . . . . . 13

2.4 .2 Kalman . . . . . . . . . . . . . . . . 15

2.4.3 Least Mean Square (LMS) algorithm . . . . . . . 17

III Adaptive Filters Research $\ldots \ldots \ldots \ldots \ldots$

3.1 Recursive Adaptive Filters . . . . . . . . . . . . . 19

3.2 Parallel Adaptive Filters . . . . . . . . . . . . . . . 21 
IV The Parallel Binary Structured LMS Algorithm . . . . . . . . . . . 26

4.1 Derivation . . . . . . . . . . . . . . . 26

4.2 Example ........................... 35

4.3 Convergence ....................... 37

V Archilectural Tradeoff of Parallel Binary Structured LMS Algorithm Blocks . 44

5.1 Evaluation of the X block: . . . . . . . . . . . . 44

5.2 Evaluation of the $\mathrm{C}$ block: $\ldots \ldots \ldots \ldots \ldots \ldots$

5.3 Evaluation of the $\mathrm{P}$ block: $\ldots \ldots \ldots \ldots \ldots \ldots$

5.4 Evaluation of the $\Delta$ block: $\ldots \ldots \ldots \ldots$. . . . . . . . . . 69

5.5 Evaluation of overall PBS $\perp$ MS performance using different P's, C's, and D's methods: . . . . . . . . . . . . . 83

5.6 Example, implementation of PBS $\_8$ for $s=4$ and $M=8 \ldots \ldots 1$

VI Comparison of Binary Structured LMS algorithm, parallel LMS, and Fast Exact LMS . . . . . . . . . . . . . . . . . . . . 95

6.1 Parallel LMS (PLMS) . . . . . . . . . . . . . . . . . 95

6.2 Fast Exact LMS (FELMS) $\ldots \ldots \ldots \ldots \ldots$

6.3 Comparison of the performance of PBS LMS architectures with P.LMS, and FELMS $\ldots \ldots \ldots \ldots \ldots \ldots \ldots \ldots \ldots$

VII Architectural Implementation of PBS LMS Algorithm Using VHDL . . . . 118

7.1 General Description of the Parallel Adaptive Filer Chip . . . . . . . . 119

7.2 Test Bench for the PAFIC . . . . . . . . . . . . . . . . . . 121

7.3 PBS.2 implementation $\ldots \ldots \ldots \ldots \ldots \ldots \ldots . \ldots \ldots$

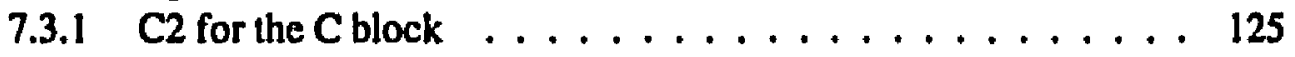

7.3.2 P2 for the P block . . . . . . . . . . . 126

7.3.3 D5 for the $\Delta$ block . . . . . . . . . . . . 127

7.3.4 Timing Diagrams of the PBS_.2 simulation . . . . . . . 129

VIII Conclusion and Future Works $\ldots \ldots \ldots \ldots \ldots \ldots$

APPENDICES 
A Time and area estimation of the Fast Exact LMS algorithm . . . . . . . 139

BIBLIOGRAPHY $\ldots \ldots \ldots \ldots \ldots \ldots \ldots \ldots \ldots \ldots \ldots$ 


\section{LIST OF FIGURES}

FIGURE

PAGE

I Block Diagram of an Adaptive Filter ... . . . . . . . . 6

2 Block Diagram of a Digital Adaptive Filter of Order $M$. . . . . . . . 7

3 Unknown System Identification . . . . . . . . . . . . . 8

4 Block diagram of a noise canceller system . . . . . . . . . . . . 10

5 Block diagram of a signal predictor ................ 11

6 Block Diagram of the Adaptive Filter using Binary algorithm . . . . . . 35

7 Convergence of the PBS $\perp$ MS algorithm for different eigenvalue spread, when gradient step size is constant $\mu=0.005, M=16, s=8 \ldots 39$

8 Convergence of the PBS $\perp$ MS algorithm for different gradient step size, when eigenvalue spread is constant $\Lambda=50, M=16, s=8 \ldots 40$

9 The average of square of the errors of two adaptive filters based on PBS LMS and original LMS algorithms ............. 4I

10 The average of square of the errors of two adaptive filters based on PBS.LMS and original LMS algorithms for the first 200 samples . . . .

11 The average of square of the errors of two adaptive filters based on PBS_LMS and original LMS algorithms for samples over $200 \ldots \ldots$

12 The outputs of two adaptive filters based on PBS.LMS and original LMS algorithms ......................... 43

13 The difference between the outputs of two adaptive filters based on PBS.LMS and original LMS algorithms $\ldots \ldots \ldots \ldots$ 
$14 \mathrm{C}$ block configuration for direct calculation, $\mathrm{Cl}$ method $\ldots \ldots \ldots$. . . 46

$15 \mathrm{C}$ block configuration for recursive calculation $\ldots \ldots \ldots \ldots \ldots$

16 Block configuration for $\mathrm{C} 2$ method $\ldots \ldots \ldots \ldots \ldots$

17 Two configurations for an s-number adder (a) s-number adder without multiplexors (b) s.number adder with multiplexors . . . . . . . 50

18 Block configuration of $\mathrm{C} 3$ method $\ldots \ldots \ldots \ldots \ldots \ldots$

19 Block configuration of $\mathrm{C} 4$ method $\ldots \ldots \ldots \ldots \ldots \ldots$

20 Block configuration of $\mathrm{C5}$ method $\ldots \ldots \ldots \ldots \ldots \ldots$

21 Configuration of one of the diagonal of the $\mathrm{C}$ block . . . . . . . 57

22 Comparison among the different C block's methods. (a) Total time delay for the $\mathrm{Cblock}$ (b) Total area required for the $\mathrm{C}$ block (c) Time area presentation

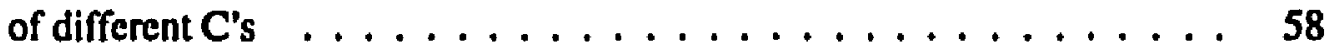

23 Comparison among the different $\mathrm{C}$ block's methods versus order of the filter. (a) Total time delay for the $\mathrm{C}$ block. (b) Total area required for the $\mathrm{C}$ block. (c) Time_area presentation of different C's. . . . . . . . . 60

24 P block configuration . . . . . . . . . . . . . 62

25 P1 block configuration for one of $p(s i)$ 's. . . . . . . . . . . 64

26 P2 block configuration for one of $p(s i)$ 's. . . . . . . . . 65

27 Block configuration of the $\mathrm{P} 3$ method $\ldots \ldots \ldots \ldots 6$

28 Block configuration of the $\mathrm{P} 3$ method $\ldots \ldots \ldots \ldots \ldots$

29 Comparison among the different $P$ block's methods for constant order of the filter, $M=128$. (a) Total time delay for the $\mathrm{P}$ block versus step size s. (b) Total area required for the $P$ block versus step size $s$. (c) Time_area presentation of different P's versus step size $s \ldots \ldots \ldots \ldots \ldots \ldots$

30 Comparison among the different $P$ block's methods for constant step size, $s=8$. (a) Total time delay for the P block versus order of the filter (b) Total area required for the $P$ block versus order of the filter (c) Time_area presentation of different P's versus order of the filter . . . . . . 70 
31 Block diagram of the $D 1$ method $\ldots \ldots \ldots \ldots \ldots \ldots \ldots$

32 Block diagram of the $\mathrm{D} 2$ method $\ldots \ldots \ldots \ldots \ldots \ldots$

33 Block diagram of the $\mathrm{D} 3$ method $\ldots \ldots \ldots \ldots \ldots \ldots \ldots$

34 Block diagram of the $\mathrm{D} 4$ method $\ldots \ldots \ldots \ldots \ldots \ldots$

35 G structure to calculate $g$ (si)'s recursively; (a) A Diagonal Element; (b) A Non_Diagonal Element; (c) Overall Recursive Structure to calculate $g(s i)$ 's

36 Comparison among the different $\Delta$ block's methods for constant order of the filter, $M=128$. (a) Total time delay for the $\Delta$ block versus step size s. (b) Total area required for the $\Delta$ block versus step size s. (c) Time_area presentation of different $D$ 's versus step size $s \ldots \ldots \ldots \ldots \ldots$

37 Comparison among the different $\Delta$ block's methods for constant step size, $s=8$. (a) Total time delay for the $\Delta$ block versus order of the filter, (b) Total area required for the $\Delta$ block versus order of the filter, (c) Time_area presentation of different $D$ 's versus order of the filter . . . . . . .

38 Comparison among the different $C$ and $P$ block's methods for constant order of the filter; (a) Comparison of C's and P's when $M=128$; (b) Comparison of $C$ 's and P's when $M=64 \ldots \ldots \ldots \ldots \ldots$

39 Comparison among the different $C$ and $\mathrm{P}$ block's methods for constant order of the filter; (a) Comparison of C's and P's when $M=128$; (b) Comparison of C's and P's when $M=64 \ldots \ldots \ldots \ldots \ldots \ldots$

40 Comparison among the different PBS LMS methods versus step size for constant filter's order, $M=128$. (a) Total time delay for the PBS $L M S$ methods versus step size; (b) Total area required for the PBS $\perp M S$ versus step size; (c) Time_area presentation of different PBS_LMS versus step size

41 Comparison among the different PBS LMS methods order of the filter, for constant step size, $s=8$. (a) Total time delay for the PBS.LMS methods versus order of the filter; (b) Total area required for the PBS.LMS versus order of the filter; (c) Time_area presentation of different PBS_LMS versus order of the filter . . . . . . . . . . . . . . . .

42 The configuration of the $\mathrm{C}$ block of PBS_8 architecture for an adaptive filter of order 8 and parallel step size $4 \ldots \ldots \ldots \ldots \ldots$ 
43 The configuration of the P block of PBS.8 architecture for an adaptive filter of order 8 and parallel step size $4 \ldots \ldots \ldots \ldots$

44 The systolic structure to calculate $g$ coefficients in the PBS.8 architecture for an adaptive filter of order 8 and parallel step size 4 . . . . . . . .

45 The configuration of the $\triangle$ block of PBS.8 architecture for an adaptive filter of order 8 and parallel step size $4 \ldots \ldots \ldots \ldots \ldots \ldots$

46 Time, area and time.area performance of P LMS algorithm versus order of the filter; (a) Total time delay per sample for PLMS algorithm; (b) Total area required for PLMS algorithm; (c) Time_area performance of PLMS algorithm. . . . . . . . . . . . . . .

47 Comparison between the different methods of implementing the FELMS algorithm versus block size s. (a) Total time delay per sample for FELMS algorithm. (b) Total area required for FELMS algorithm. (c) Time_area presentation of different FELMS methods . . . . . . . . . 100

48 Comparison between the different methods of implementing the FELMS algorithm versus order $M$. (a) Total time delay per sample for FELMS algorithm. (b) Total area required for FELMS algorithm. (c) Time_area presentation of different FELMS methods . . . . . . . . . . 102

49 Comparison between the different methods of implementing the PBS.LMS and P.LMS versus step size s. (a) Total time delay per sample; (b) Total area required; (c) Time_area performance of different methods . . . . . .

50 Comparison between the different methods of implementing the PBS LMS and PLMS versus order of the filter. (a) Total time delay per sample; (b) Total area required; (c) Time_area performance of different methods . . .

51 Comparison between the different methods of implementing the PBS.LMS and FELMS versus step size $s$. (a) Total time delay per sample; (b) Total area required; (c) Time_area performance of different methods . . . . . . 108

52 Comparison between the different methods of implementing the PBS $\perp M S$ and FELMS versus order of the filter; (a) Total time delay per sample; (b) Total area required; (c) Time_area performance of different methods. . . . 110 
53 Comparison between the performance of the PBS 2, PBS.8, FELMS, and P.LMS versus step size s. (a) Total time delay per sample; (b) Total area required; (c) Time_area performance of different methods . . . . . . .

54 Comparison between the performance of the PBS 2, PBS.8, FELMS, and PLMS versus order of the filter. (a) Total time delay per sample; (b) Total area required; (c) Time_area performance of different methods . . . . . . 114

55 Comparison between the speed up of the PBS 2, PBS.8, and FELMS over PLIMS; (a) Speed ups versus step size s; (b) Speed ups versus order of the filter.

56 The average of the errors of two adaptive filters based on PBS_8 and P L LMS algorithms $\ldots \ldots \ldots \ldots \ldots \ldots \ldots \ldots$

57 More delails of the average of the errors of two adaptive filters based on PBS.8 and PLMS algorithms . . . . . . . . . . . 117

58 The over all layout of PAFIC . . . . . . . . . . . . . . . 119

59 Blocks and Signals inside PAFIC . . . . . . . . . . . . . . . 122

60 Blocks of the test bench of PAFIC . . . . . . . . . . . . . . . 124

61 The Configuration of Multipliers in the $C$ Block for circuit with parallel step size equal 8; (a) Multipliers and Registers configuration; (b) One Multiplier. 126

62 Adders, Latches, and Multiplexors required in the s_number adders of the C block for each $c$ coefficient, $s=8, \ldots \ldots \ldots \ldots \ldots \ldots$

63 Circuit to calculate one of $p(s i)$ 's in the P block, using P2 architecture. . . 128

64 The structure of the $\Delta$ block usinf D5 architecture; (a) A Diagonal element of the lower part; (b) A Non_diagonal element of the lower part; (c) Over all recursive structure of the $\Delta$ block, calculating $g(s i)$ 's, $\operatorname{dwv}(s i)$ 's, and $w(n+s)$ 's. . . . . . . . . . . . . . .

65 The circuit which calculates $\operatorname{dwv}(s i), s i=0,1, \ldots s-1$ and $w(n+s)$ in the $\Delta$ block for an adaptive filter of order $M . \ldots \ldots \ldots \ldots 131$

66 Timing diagram of a RESET cycle $\ldots \ldots \ldots \ldots \ldots \ldots \ldots$

67 Timing diagram of a FILTERING cycle $\ldots \ldots \ldots \ldots$. . . . . . . . 133 
68 Timing diagram of a W_WRTTE cycle $\ldots \ldots \ldots \ldots \ldots$

69 Timing diagram of a W.READ cycle $\ldots \ldots \ldots \ldots \ldots \ldots$ 


\section{LIST OF TABLES}

TABLE

PAGE

I The Kalman filter algorithm ................. 17

2 Lcast Mean Square (LMS) algorithm . . . . . . . . . . . . 18

$3 \quad$ Terms of $\Delta w(n+4) \ldots \ldots \ldots 32$

$4 \Delta w(n+4)$ in terms of $\hat{x}, p$, and $c \ldots \ldots . \ldots . \ldots . \ldots$

5 Comparison of the complexity and time delay for the $\mathrm{C}$ block calculation . 55

6 Comparison of the four method to implement the P block . . . . . . 66

7 Required multipliers and adders and number of iterations, $T_{D 4}$, to calculate $\Delta w(n+s)$ using D4 method. . . . . . . . . . . . . . 76

8 Comparison of the five methods to implement the $\Delta$ block . . . . . . . . 78

9 Description of the input and output signals of PAFIC . . . . . . . . 120 


\section{CHAPTER I}

\section{Introduction}

Iterative solutions to the adaptation process are slow and time consuming methods. Each iteration requires a huge amount of calculation. Many attempts have been made to speed up this process. Technology allows many processing elements on a single chip, encouraging a parallel architeclure to solve the adaptation process. However a parallel structure is more complicaled than an itcrative one. Such a complex circuit limits development of a paraliel structure for an adaptive filter.

In this dissertation, a parallel algorithm for adaptive filters suitable for VLSI implementation is presented. Based on a direct relation between the binary presentation of numbers with $s$ digits and the terms needed to calculate the difference vector, a parallel struclure for an adaptive filter is obtained which is an exact representation of the original LMS algorithm. Using this parallel algorithm, the tap weights of an adaptive filter at time $n+s$ are calculated in terms of the tap weights at time $n$, inputs from time $n$ to time $n+s-1$, and desired outputs from time $n$ to time $n+s-1$.

The proposed parallel algorithm is a simple but versatile algorithm for adaptive filters. This algorithm is suitable for implementation on a VLSI chip. Such an implementation cannot be achieved without a regular and modular architecture. The parallel algorithm presented has a regular and modular structure such that it is capable of enhancing the 
number of parallel steps, as well as the order of the filter, without significantly increasing the complexity of the circuit.

This research can be partitioned into three major stages. The first stage is to develop a parallel algorithm for adaptive filters. In doing so, the other parallel algorithms for the adaptive filters which fulfill the objectives mentioned above are researched. This is accomplished through survey and evaluation of algorithms presented in the current literature. The parallel binary structured LMS is developed through out this stage. The area, time, and the area-time product are used as measures of merit in evaluation of the algorithms. Component count, such as adders and multipliers, is used as appropriate to estimate the area required to build the parallel adaptive fitter. Correspondingly, component delay is used to estimate the time. The algorithms are also cvaluated in regards to the ability to use regular and modular structures in a VLSI implementation. The ability to partition the VLSI architecture into major sub-elements is also evaluated.

In the second stage of the research, the impact of the pipelining in implementing the presented parallel algorithm is evaluated. In order to achieve the maximum possible speed, the use of the pipelining in the implementation of the parallel structure is investigated. Pipelining is applied to the whole structure, by pipelining the blocks of the parallel architecture. Pipelining is also applied to the circuit of each block. Since pipelining will increase the design time, as well as the area of the chip, its effect on the design of each block is evaluated.

The third stage of our research is to evaluate the presented parallel binary structured algorithm for a VLSI implementation. The most time and area-time efficient architectures 
are mapped to corresponding VHDL architectures, using IEEE 1076-1987 VHDL (VHSIC (Very High Speed Integrated Circuit) Hardware Description Language). VHDL is used to verify and validate the architectures at a high level of abstraction using a behavioral model. The VHDL description is modified to a structural description employing the regular and modular structures identified in the first stage.

Regularity and modularity, two important factors for VLSI implementation, exist in the presented binary structured algorithm, A custom design adaptive filter, with different degrees of update step size and different orders of the filter, can be designed. The performance of the proposed binary structured LMS algorithm is compared to the performance of the parallel LMS and Fast Exact LMS algorithms. This comparison shows that the PBS_LMS algorithm is inherently more parallel than the parallel LMS and the Fast Exact LMS algorithm.

The dissertation is organized as follows. Chapter two contains an introduction to adaptive filters, their applications, and three of their classical solutions. The third chapter consists of a summary of the relevant literatures on the recursive and parallel algorithms for adaptive filters.

Starting from chapter four, the results of our research on developing a parallel algorithm for adaptive filters is presented. Chapter four contains the derivation and explanation of our parallel binary structured LMS algorithm for adaptive filters, which is called the PBS.LMS algorithm. Chapter five contains the architectural trade-off evaluations for different implementations of the PBS.LMS algorithm. Comparing the different architectures of the $C, P$, and the $\Delta$ block, two optimum architectures for each block are selected. These 
architectures are used to implement eight PBS $L M S$ based adaptive filters. Three measures of merit, the area, time, and time_area product are applied to the eight different architectures of the PBS LMS algorithm for different parallel step sizes when the order of the adaptive filter is constant. The same measures of merit are applied to the eight different architectures of the PBS LMS algorithm when the parallel step size is constant and the order of the adaptive filter is varied.

In chapter six, the performance of the PBS.LMS algorithm is compared to the performance of two other parallel LMS algorithm, P_LMS and FELMS. Chapter seven contains the results of the VHDL simulation of the PBSLMS algorithm. Chapter eight has the conclusion of the dissertation and suggestions for the future work in this line of research. 


\section{CHAPTER II}

\section{Adaptive Filters}

Digital adaptive filters are a class of filters whose characteristic can be changed dynamically. Using specific properties of the output signal, the system changes its characteristic function in such a way that for any input, the oulput will have the desired properties. When the actual output of the filter differs from the expected output, the difference can be used to adapt the characteristic function of the filter to obtain better results, i.c., less error. Figure I shows the block diagram of an adaptive filter.

\subsection{Definition of Adaptive Filters}

An FIR digital filter of order $M$ contains $M$ tap weights, which are usually pre-determined and unchangeable. A corresponding digital adaptive filter, as depicted in Figure 2, has $M$ variable tap weights adjusted by its controller. A new input value is applied to the filter at each time step and the output is determined by the present tap values. The controller compares this output with the desired value for the output. The difference between the desired output and the actual output is the estimation error of the filter. The controller calculates new values for the taps in such a way that a criterion related to the estimation error, expressed through the performance index, approaches its minimum. The purpose of the adaptation process is to minimize this performance index. Many algorithms for this 


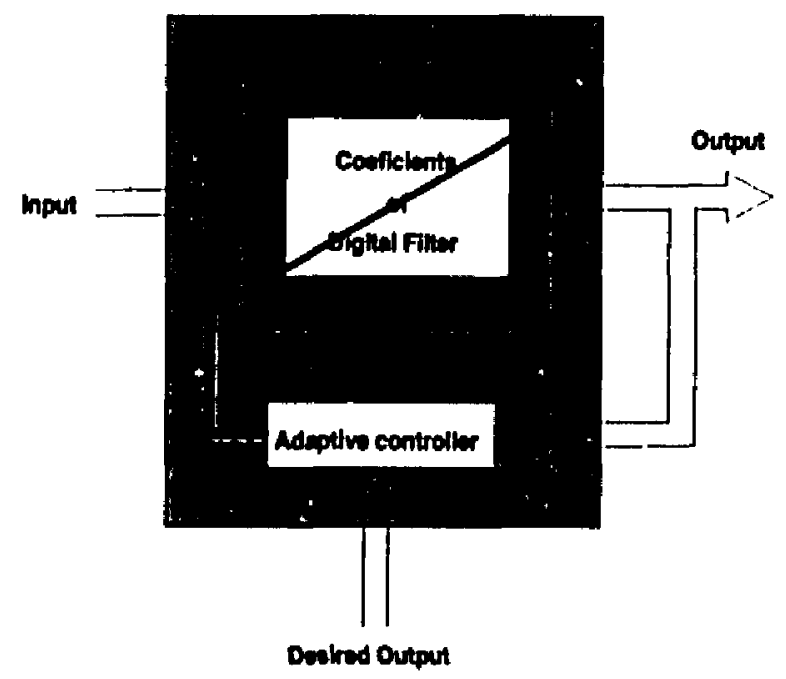

Figure 1: Block Diagram of an Adaptive Filter

minimization have been developed based on different criteria.

Because of their adjustable characteristics, adaptive filters are very powerful and useful devices in many fields of engineering such as control, radar, communication, image processing, seismology, sonar and pattern recognition systems. Using adaptive filters, the characteristics of an unknown digital system can be identified, noise in data transmission lines can be canceled, and future or past inputs to a system can be predicted. 


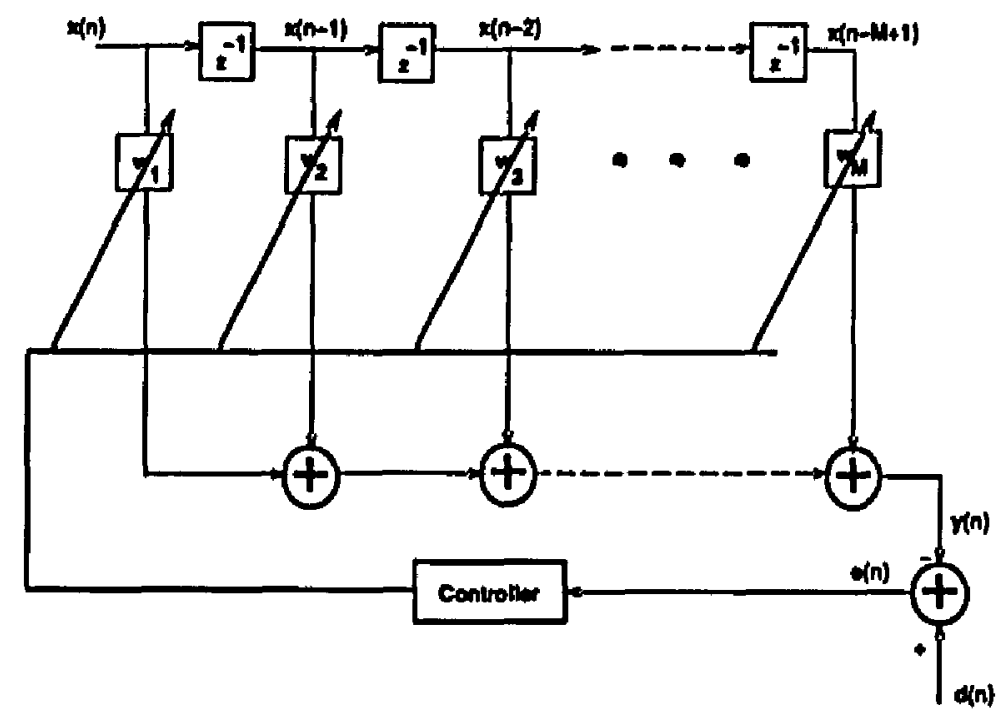

Figure 2: Block Diagram of a Digital Adaptive Filter of Order $M$

\subsection{Some Applications For Digital Adaptive Filters}

As mentioned, adaptive filters are very powerful and useful devices in many fields of engineering such as communication, control, radar, image processing, seismology, sonar and pattern recognition systems. In this section three applications of adaptive filters are briefly described. Each description contains an illustration and a short discussion. These descriptions have been presented to clarify the idea of adaptive filters and their applications.

\subsubsection{Unknown system identification}

We have an unknown system, and we want to construct a model for it. An adaptive filter is very useful device to model an unknown system with a digital filter. Fig. 3 shows a block 


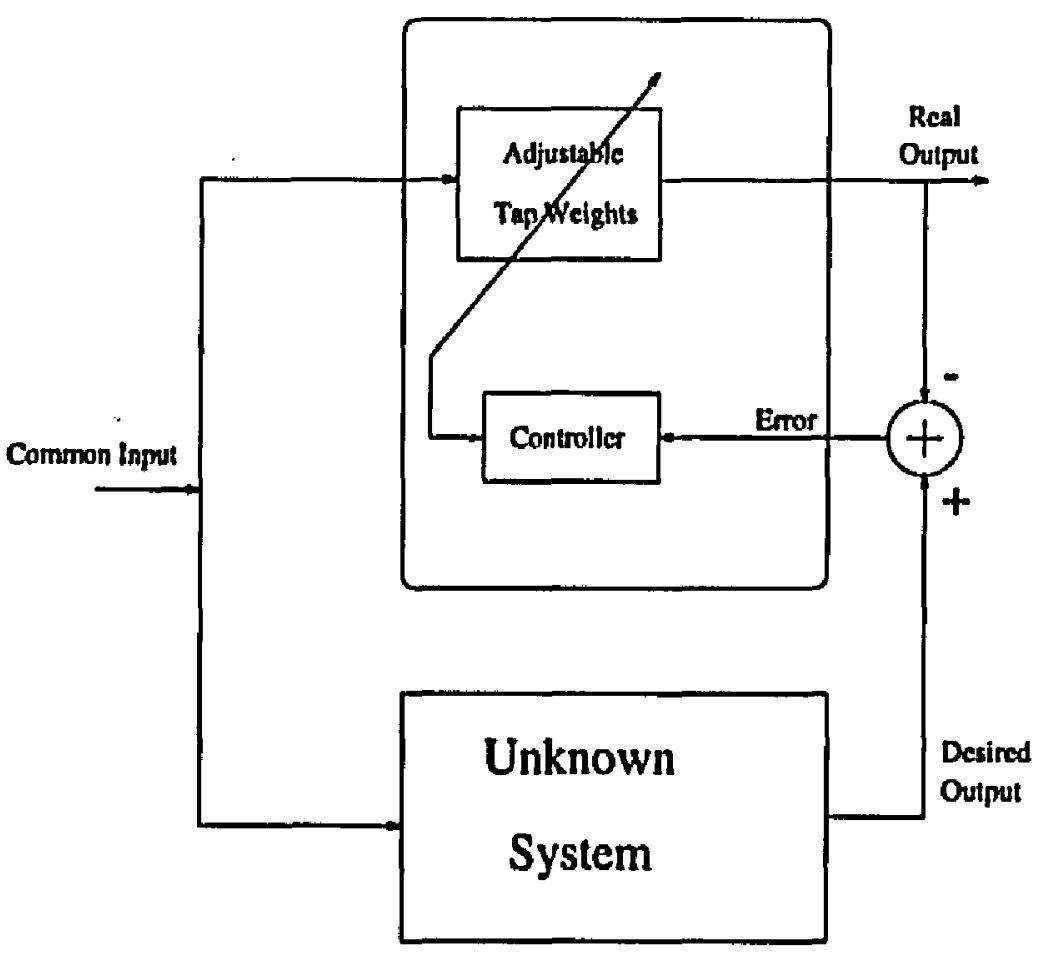

Figure 3: Unknown System Identification

diagram of an unknown system identification.

Consider an adaptive filter of order $\mathrm{M}$. It is desired to adjust the tap weights of this filter in such a way that the unknown system can be approximated by this filter. As a result, the response of the unknown system to an input signal can be predicted by the adaptive filter.

Initially an arbitrary values will be assigned to the tap weights of the filter. Then, in each iteration, a common input is applied to both the unknown system and the adaptive filter. The output of the unknown system is considered as the desired output for the adaptive filter. The difference between this desired output and the real output of the adaptive filter, 
the estimation error, will be considered as a function of filter tap weights. Finally the controller computes the new values for filter's taps to minimize a performance index. The performance index is a function of the estimated error. Continuing this process for some ilerations, the optimum values for the taps weights are obtained. A digital filter with such values in its taps weights is the model of the unknown system.

\subsubsection{Noise cancellation in the data transmission line}

Transmission of digital signals through an electrical channel to a recciver is almost always associated with noise. For detecting and canceling this noise, an adaptive filter is applicable. A block diagram of a noise canceller in a transmission line is illustrated in Fig. 4

Test signals are generated at the transmitter and receiver by two identical test signal generators. Obviously the generator at the receiver considers the delay time caused by the transmitting channel in order to synchronize its signal with that generated by the generator at the transmitter. As a result, at the receiver side a test signal and the transmitted pattern of that signal are available simultaneously. The adaptive filter constructed at the receiver employs these two signals, one as the input and the other as the desired output, to adjust its coefficients in order to minimize the effect of channel noise on the test signal.

The noise canceller operates in two modes, the training mode and the tracking mode. Two synchronous switches at the transmitter and at the receiver control these two states of the system. In the training mode the test signal from the Test Signal Generator 1 is fed to the transmitter to be sent through the channel to the receiver. In the receiver side the Test Signal Generator 2 produces the same signal as the Test Signal Generator 1 has produced. Obviously the input signal to the receiver differs from the original signal because of the 


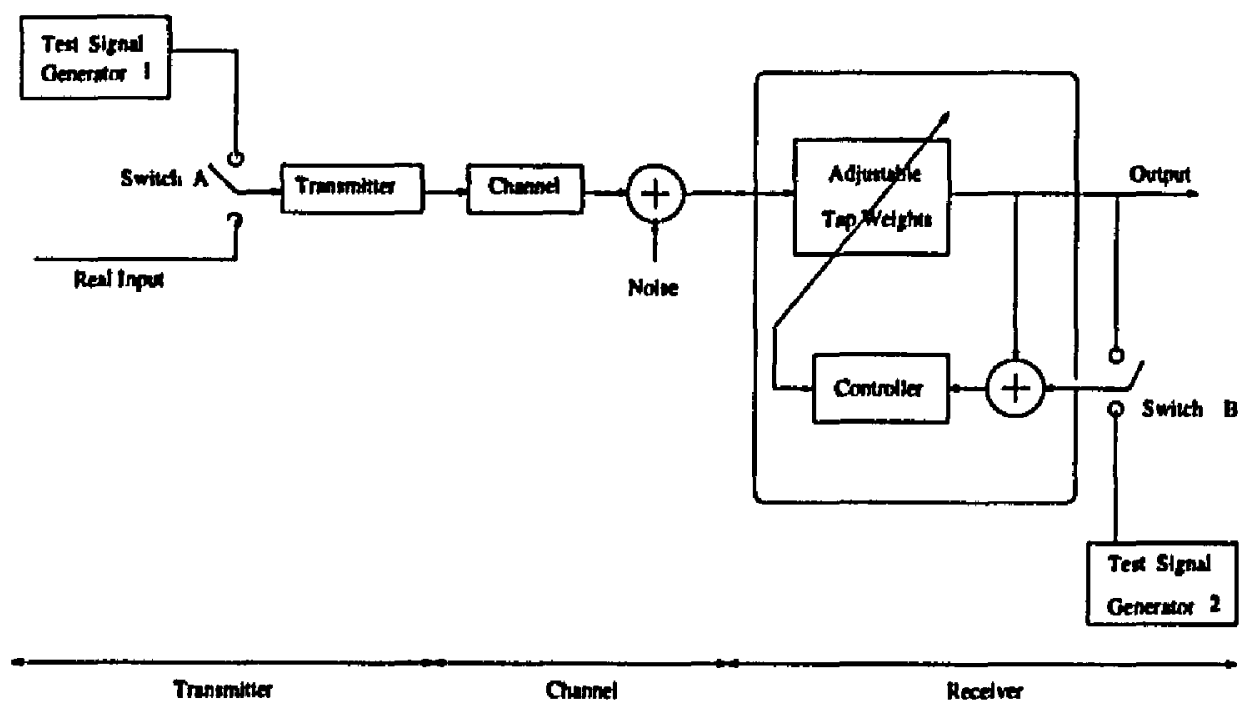

Figure 4: Block diagram of a noise canceller sysiem

noise of the transmission line. The adaptive filter receives the noisy signal from the line as its input, and the original signal from the generator at the receiver side as the desired output. The controller adjusts the taps values of the adaptive filter to climinate the effect of noise. By loading the new values into the taps, the training mode ends. In the tracking mode the real data are transmitted through the channel into the receiver. The adaptive filter corrects the received data by its characteristic function. This correction on the received data is considered as the noise cancellation, based on the assumption that the probability of noise contribution in the two modes are equal. As a result, the adaptation in the training mode is used in the tracking mode to cancel the noise effect on the real signal. Therefore the most accurate data are obtained at the output of receiver. 


\subsubsection{Signal Prediction}

In this application the adjustable filter operates as a predictor. That is the adaptive filter uses previous input signals in order to predict the next input signal. In other words by using $M$ past inputs, the tap gains will be set at the (n-1)th time step in such a way that the input at the time $n$ can be predicted. Afterward, the $(n)$ th input is compared with the predicted signal to determine the estimation error. In turn, this error is used by the controller to adjust the tap weights properly. Fig. 5 shows the block diagram of a forward predictor. The signal predictor is used in many practical applications such as speech digitization and echo cancellation.

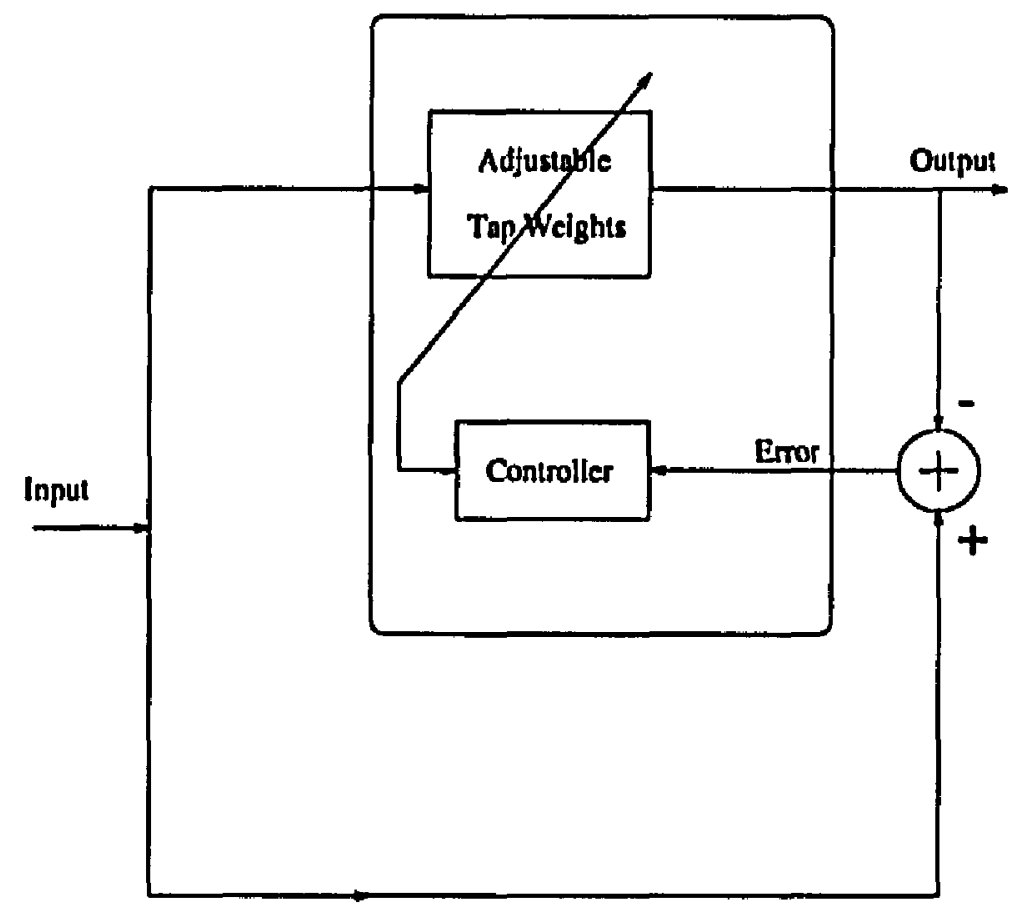

Figure 5: Block diagram of a signal predictor 


\subsection{Performance Index Minimization}

In computing the tap weights, the objective is minimization of the difference between the actual output and the desired output, the performance index. Consider a transversal adaptive filter of order $M$, as depicted previously in Fig. 2. The output of this filter at time $n$ is

$$
y(n)=x^{\prime \prime}(n) w(n)
$$

where $\mathrm{x}(n)$ is an $M$-by-1 input vector at time $n$, i.c.,

$$
x(n)^{T}=[x(n) x(n-1) \ldots x(n-M+1)],
$$

$w(n)$ is the tap weights vector at that time, i.c.,

$$
w(n)^{r}=\left[w_{1}(n) w_{2}(n) \ldots w_{M}(n)\right]
$$

$H$ denotes the Hermitian transpose of a vector. If $d(n)$ denotes the desired output at time $n$, then the error at this time is defined as

$$
c(n)=d(n)-y(n)
$$

Based on the application of the adaptive fitter, different definitions of the performance index are used. One definition of the performance index is the expectation of the square of the error at each time, or

$$
\varepsilon(n)=E\left(|e(n)|^{2}\right)
$$

Another way to define the performance index is the sum of the square of all errors since the beginning of the process, i.e.,

$$
\varepsilon(n)=\sum_{i=1}^{n}|e(n)|^{2}
$$


We also can define a performance index as the sum of squares of the $M$ previous errors, as

$$
\varepsilon(n)=\sum_{i=n-M+1}^{n}|c(n)|^{2}
$$

In the time varying environment, the performance index can be defined as a function of the $M$ previous errors along with a forgetting factor, $\lambda$. This factor magnifies the importance of the new errors and diminishes the effects of the older ones, i.c.,

$$
\varepsilon(n)=\sum_{i=n-M^{\prime}+1}^{n} \lambda^{n-i}|c(n)|^{2} .
$$

The performance index at each time step is a function of the tap weights and can be denoted as $\varepsilon(n, w)$, or $\varepsilon(w)$.

\subsection{Adaptive Filter Algorithms}

The adaptation process has been formalized and many algorithms for adaptive filters, for different types of signals and systems, have been developed. In this part, three classical methods which are frequently used in implementing adaptive filters are described. More solutions for adaptive filters can be found in [1]-[4].

\subsubsection{Wiener}

Wiener theory is based on minimization of the expectation of the Mean Squares of Error (MSE). In the Wiener filter, the performance index is

$$
\varepsilon(w)=E\left(|e(n)|^{2}\right)
$$


where the estimation error $c(n)$ is defined in 2.4 and $E$ is the expectation operator. Using 2.1 and 2.4 the estimation error is

$$
c(n)=d(n)-w^{H}(n) \times(n)
$$

or

$$
c^{*}(n)=d^{*}(n)-x^{\prime \prime}(n) w(n)
$$

where * denotes complex conjugate operation. Sinee the performance index is equal to $E\left(|c(n)|^{2}\right)$, in the complex number notation we can rewrite the performance index as

$$
\varepsilon(w)=E\left(c(n) c^{*}(n)\right)
$$

By expanding the right side of 2.12 , we have

$$
\varepsilon(w)=E\left[\left(d(n)-w^{\prime \prime}(n) x(n)\right)\left(d^{*}(n)-x^{\prime \prime}(n) w(n)\right)\right]
$$

or

$$
\begin{aligned}
\varepsilon(w)= & E\left(d(n) d^{*}(n)\right)-w^{\prime \prime}(n) E\left(d^{*}(n) \times(n)\right)- \\
& E\left(d(n) x^{\prime \prime}(n)\right) w(n)+w^{\prime \prime}(n) E\left(x(n) x^{\prime \prime}(n)\right) w(n) .
\end{aligned}
$$

To obtain the minimum of the performance index, 2.14 is differentialed with respect to $\mathrm{w}(n)$ and set the result equal to zero.

$$
\delta \varepsilon(\mathrm{w}) / \delta \mathrm{w}=-2 E\left(d^{*}(n) \mathrm{x}(n)\right)+2 E\left(\mathrm{x}(n) \mathrm{x}^{\prime \prime}(n)\right) \mathrm{w}(n)=0
$$

Thus

$$
E\left(\mathrm{x}(n) \mathrm{x}^{\prime \prime}(n)\right) \mathrm{w}(n)=E\left(d^{*}(n) \mathrm{x}(n)\right)
$$


We define the autocorrelation matrix of the input vector

$$
\mathbf{R}(n)=E\left(\mathbf{x}(n) \mathbf{x}^{\prime \prime}(n)\right),
$$

and the cross-correlation vector of the input vector and the desired output

$$
p(n)=E\left(d^{*}(n) \times(n)\right),
$$

then 2.16 can be rewrilten as

$$
\mathbf{R}(n) \mathbf{w}(n)=\mathbf{p}(n) .
$$

Equation 2.19 is called the normal equation with a traditional solution for the optimum lap wcights $\hat{w}$,

$$
\hat{\mathrm{w}}=\mathrm{R}^{-1}(n) \mathrm{p}(n) .
$$

That is, finding the tap weight vector at each iteration requires calculation of $\mathbf{R}^{-1}$. Inversion of an $M$ by $M$ matrix is an $O\left(M^{3}\right)$ algorithm. Other methods have been suggested in order to achieve a faster solution to the adaptation process, rather than direct calculation in equation 2.20. For instance the Kalman filter algorithm requires $O\left(M^{2}\right)$ time for each iteration, and Gradient Vector Estimation method requires $O(M)$ time per iteration.

\subsubsection{Kalman}

Kalman filter theory is based on minimization of the least mean square error. In the Kalman filter algorithm, the system measures the difference between the desired output and the actual output of the system without changing the tap weights from the last iteration. At time $n$, the tap weights are still unchanged from time step $n-1$. The actual output of system is

$$
y(n)=w^{\prime \prime}(n-1) x(n) .
$$


The difference between this output and the desired response of the system at this time is called 'the innovation.' The innovation at time $n$, denoted by $\alpha(n)$, is defined as

$$
\alpha(n)=d(n)-y(n)
$$

or

$$
\alpha(n)=d(n)-w^{\prime \prime}(n-1) x(n)
$$

This scalar value, $\alpha(n)$, accompanied with a gain vector, $g(n)$, modifies the tap weights of the previous step $w(n-1)$ to obtain the tap weights of the filter at time $n$,

$$
w(n)=w(n-1)+g(n) \alpha^{*}(n)
$$

The Kalman gain vector, $g(n)$, is defined as

$$
\mathbf{g}(n)=\mathbf{K}(n-1) \mathbf{x}(n)\left(\mathbf{x}^{H}(n) \mathbf{K}(n-1) \mathbf{x}(n)+J_{\min }\right)^{-1}
$$

The $\mathrm{K}$ matrix in 2.25 represents the correlation matrix of the weight-error vector, $w(n)-\hat{w}$, and updates at each iteration as

$$
\mathbf{K}(n+1)=\mathbf{K}(n)+\mathbf{g}(n+1) \mathbf{x}^{\prime \prime}(n+1) \mathbf{K}(n) .
$$

Thus, in each iteration, matrix multiplication of two $M$ by $M$ matrices is performed, an $O\left(M^{2}\right)$ process. $J_{\min }$ in the gain vector equation (2.25) is the minimum mean-squared error and assumes a value between 0.001 to 0.01 times the variance of the desired output.

To start this recursive process an initial value for $w$ and $K$ is considered. $w(0)$ is initiated to the null vector, $w(0)=0$, and $K(0)$ is initiated to

$$
\mathbf{K}(0)=c \mathbf{U}
$$


where $U$ is a unity matrix and $c$ is a small positive constant. Table $I$ shows the summary of the Kalman algorithm for adaptive filters.

Table 1: The Kalman filter algorithm

1.Initialize

$$
\begin{aligned}
& \mathbf{w}(0)=\mathbf{0} \\
& \mathbf{K}(0)=c . U, c>0
\end{aligned}
$$

2. For each time step $n+1$

$$
\begin{aligned}
& \alpha(n)=d(n)-\mathbf{w}^{H}(n-1) \mathbf{x}(n) \\
& \mathbf{g}(n)=\mathbf{K}(n-1) \times(n)\left(\mathbf{x}^{H}(n) \mathbf{K}(n-1) \mathbf{x}(n)+J_{\min }\right)^{-1} \\
& \mathbf{w}(n)=\mathbf{w}(n-1)+\mathbf{g}(n) \alpha^{*}(n) \\
& \mathbf{K}(n+1)=\mathbf{K}(n)+\mathbf{g}(n+1) \mathbf{x}^{\prime \prime}(n+1) \mathbf{K}(n)
\end{aligned}
$$

3. Go to step 2

\subsubsection{Least Mean Square (LMS) algorithm}

Least Mean Square algorithm is based on minimizing the Mean Square of the crrors (MSE). That is, the controller in the adaptive filter minimizes the performance index of equation 2.5 . The LMS algorithm results in the same normal equation as Wiener method does, equation 2.19. However, in this method instead of inverting the matrix $\mathbf{R}$, a recursive procedure is used to achieve the optimum tap weights. This recursive method is called Gradient Vector Estimation. Accordingly at each time step $n+1$, the tap weights vector, $w(n+1)$, is calculated using the estimation error and the input vector at the previous time $n$. Therefore

$$
w(n+1)=w(n)+\mu x(n) e^{*}(n)
$$

The step size, $\mu$, is a positive parameter limited to

$$
0<\mu<2 / \text { (total input power) }
$$


where

$$
\text { total input power }=\operatorname{Mr}(0) \text {. }
$$

$r(0)$ is the variance of input signal at time $n$, i.c.,

$$
r(0)=E\left[x(n) x^{*}(n)\right]
$$

Assuming an initial condition $w(0)=0$, an iterative process can be used to computc the tap weights. A summary of the LMS algorithm is shown in Table 2.

Table 2: Lcast Mean Square (LMS) algorithm

\section{Initialize}

2. For each time step $n+1$

$$
w(0)=0
$$

3. Go to step 2

$$
\begin{aligned}
& c(n)=d(n)-w^{\prime \prime}(n) \times(n) \\
& w(n+1)=w(n)+\mu \times(n) c^{\prime \prime}(n)
\end{aligned}
$$

The two vector multiplications require $O(M)$ time. Direct implementation of equation 2.28 results in a small circuit, however, for a large number of inputs, extensive time is required as the circuit is constrained to perform each iteration. The linear recursive relation in equation 2.28 has the capability to be enhanced by a look-ahead formula for the tap weights calculation, reducing computation time. 


\section{CHAPTER III}

\section{Adaptive Filters Research}

Increasing the applications of the adaptive filters in different fields of engineering leads to more research on this type of digital filter. The first line of research on adaptive filters was focussed on the recursive solutions. Then, when VLSI technology allowed more hardware on chips, parallel algorithms for adaptive filters were developed.

\subsection{Recursive Adaptive Filters}

In the past decade, much research has been done on recursive algorithms for the adaptation process, suitable for the available lechnology and demand.

A single chip VLSI archilecture for an adaptive equalizer is described in [5]. This chip can handle filtering of digital transmission lines at hundreds of Kbits per seconds. They used a $1.5 \mu m$ CMOS technology to develop their circuit which included several digital signal processors such as a filtering processor, an analog to digital converter, a digital phase lock loop processor, and a controller processor. The controller synchronizes the timing of the actions of the other processors, as well as memory management and input/output procedures.

A bidirectional chain VLSI system for recursive adaptive filters has been developed in [6]. In [7] authors introduce a recursive technique for the adaptation process based on the 
Gradient Vector Estimation method. In the original LMS algorithm the gradient step size is constant. This constant is limited such that it guarantees the convergence of the algorithm. In their proposed algorithm, the authors increase the convergence speed of the algorithm by calculating the optimum gradient slep size at each iteration. This algorithm can be applied to either lattice or transversal adaptive filters. Although their original concern is not the VLSI implementation of adaptive fillers, the proposed algorithm can readily be mapped to a VLSI implementation. In each of these papers, the algorithm and implementation is based upon a recursive solution. Another example of recursive solutions may be found in [8]. In this paper, a sequential Least Square (LS) estimation algorithm, applicable in filtering and prediction, is presented.

Since Least Mean Square (LMS) and Least Square (LS) Error algorithms are widely used in different applications of adaptive filters, many researchers have focussed their effort to modify these algorithms and to speed up their convergence. Other research on these two algorithms are directed toward making them suitable for VLSI implementation and/or to implement them on a chip.

The authors in [9] proposed a time varying gradient step size, $\mu$, for gradient method estimation. Their aim is to improve the convergence of the LMS algorithm by assigning a more accurate value to the $\mu$ at each time. In [10], the author presents a through investigation and comparison between LMS and Normalized LMS, NLMS, algorithms. Usually LMS has a constant gradient step size. On the other hand NLMS has a step size which is normalized at each time step by the norm of the input vector. That is

$$
\mu_{k}=\frac{\mu}{\|x(n)\|}
$$


The author shows that the convergence of the NLMS is faster than the original LMS. He also presents that NLMS is not only stable on the average, like LMS, but it is stable at every sample times. Having all these advantages, NLMS is not as popular as adaptive filter algorithm as the original LMS is. The author concludes that the division operation in the normalization of the gradient step size of NLMS is the main reason that the designers use LMS much more often than NLMS algorithm in their DSP applications.

Authors in [11] present an implementation of the LMS algorithm without using a multiplication operation. Instead, they propose to use memory aceessing, adding, shifting, and scaling the numbers by integer power of 2 operations. They implemented their adaptive filter circuit using only these operations. A bidirectional chain structure for adaptive filters suitable for VLSI implementation is presented in [12].

\subsection{Parallel Adaptive Filters}

In recent years, the rapid evolution of VLSI technology has provided a dramatic increase in circuit density and performance. These increases accommodate increased functionality and can be used to implement systems that were previously unrealizable. Implementation of digital adaptive filters on a single chip is common; implementation of parallel algorithms for adaptive filters is possible.

Recursive solutions result in implementations on either a single-chip or a small number of chips. Multi-chip modules, the construction of a module from multiple die mounted on a larger silicon wafer substrate, provide the capability to readily implement parallel algorithms. Several parallel algorithms have been developed. In [13] a block implementation of an adaptive filter was proposed that uses a parallel form of the Wiener method. Another 
parallel structure has been presented in [14]. Here, the least square method is used to build an IIR adaptive filter.

Authors in [15] propose a structure to implement a pipelined and parallel adaptive filter. Their implementation is based on a word-level pipeline and block realization. The block realization provides for look-ahead computation within a typical lattice structure architecture. To obtain a high-speed area-efficient filter, they use extensive pipelining and parallelization of the computations.

A block implementation algorithm to improve the convergence of LMS algorithm is described in [16]. In this new algorithm, the tap weights of the filter are updated $n$ times on each data received to the filter.

In [17] and [18], a look-ahead algorithm has been developed and used to implement a parallel adaptive filter. The authors examined the LMS transversal adaptive filter, in which the performanec index is

$$
\varepsilon(n)=\sum_{i=1}^{n} \lambda^{n-i}|c(n)|^{2}
$$

where $\lambda$ is the forgelting factor, a positive number less than one. The paper shows that the variables of the normal equation at time slep $n+s$ can be calculated using the variables of the normal equation at time $n$, inputs to the system from time $n+1$ to $n+s$, and the desired outputs from time $n+1$ to $n+s$. The normal equation at time $n+s$ is

$$
\Phi(n+s) w(n+s)=0(n+s)
$$

where $\Phi$ is the correlation matrix and $\theta$ is the cross-correlation vector. $\Phi$ and $\theta$ at time 
$n+s$ are formulated as

$$
\Phi(n+s)=\lambda^{\prime} \Phi(n)+\sum_{i=n+1}^{n+s} \lambda^{n-i} \mathbf{x}(i) \mathbf{x}^{\prime \prime}(i),
$$

and

$$
O(n+s)=\lambda^{\prime} O(n)+\sum_{i=n+1}^{n+s} \lambda^{n-i} \mathbf{x}(i) d^{*}(i) .
$$

The authors also present a VLSI arehitecture for implementation of their parallel algorithm.

Other research to develop a parallel structure for LMS and LS algorithms are reported. A five chip VLSI implementation of an echo canceller system is reported in [19]. The system employs an adaptive filter of order $5 / 2$ and works with input signals at $8 \mathrm{KHz}$. The values of the 512 tap weights can be updated in $125 \mu \mathrm{sec}$. This adaptive filter is based on a recursive solution as

$$
w(n+1)=w(n)+K_{n} X(n)
$$

where

$$
K_{n}=\frac{\alpha e(n)}{\sum_{i=0}^{n-1} x_{i}} .
$$

$K_{n}$ at each iteration is computed by a general purpose DSP, outside the chip. Computationally, this adaptive filter chip is not a self-sufficient unit. To implement this unit they used CMOS technology with minimum gate length of $1.5 \mu \mathrm{m}$ and typical gate delay of $1.0 \mathrm{nsec}$.

In [20], the authors present a block-update algorithm for digital adaptive equalizers based on the modified Gauss-Newton algorithm. In the proposed method the performance index is the Least Square of the errors. Since this algorithm is developed for an online equalizer, it updates the tap weights of the adaptive equalizer each every one block size, $L$, 
rather than at every step. The authors compared the performance of their proposed algorithm with one based on the Mean Square error. They show, through a compuler simulation, that their algorithm is less sensitive to the channel distortion than MSE algorithm. By comparing their equalizer with a sequential Least Square lattice equalizer, the authors show that their algorithm is superior in convergence speed and crror performance. The only disadvantage of their algorithm is the computational complexity which increases when the block size $\mathrm{L}$ increases.

The authors of [21] present a parallel architecture for Recursive Least Square adaptive filters. Another parallel architecture for adaptive filters with lattice structure is presented in [22]. In [23], the authors suggest a pipelined VLSI structure in which 125 nsec time delay for multiplication-accumulation operation is considered.

In [24] a parallel structure for the LMS algorithm is proposed. In this algorithm, the authors develop a block implementation of the LMS algorithm by rearranging the data, using matrix manipulation techniques, and deleting some redundancy in updating the tap weights of the adaptive filter. Called fast exact LMS (FELMS), FELMS has the same convergence properties as the original LMS has, with fewer operation per iteration. Another direct parallel implementation of LMS algorithm in adaptive filters is presented in [25]. An adaptive filter of order $M$ uses $M$ multiplier-accumulators plus $M-1$ parallel adders 10 converge its tap weighs to their optimum values. In this way each iteration of the adaptation process is completed in $2 T$ mult $+\left(\log _{2} N\right) T$ add. The last two proposed parallel LMS algorithms, [24] and [25], are described in the more detail in the chapter VI.

In 1991 the first general purpose DSP with parallel structure, TMS320C40, was intro- 
duced by Texas Instruments, Inc. [26]. However the complexity of adaptive filters with high volume of calculation per iteration prevented building a single purpose parallel structured adaptive filter chip. Motorola Company manufactured a totally scrial and sequential general purpose digital filter chip, 56200 [27]. This chip can be used as an adaptive filter but computationally it is not a self-sufficient unit. MOTOROLA 56200 needs a DSP, such as MOTOROLA 56000, to handle its computational tasks.

Our research is to develop a very regular and modular parallel algorithm for adaptive filters, suitable for the VLSI implementation. 


\section{CHAPTER IV}

\section{The Parallel Binary Structured LMS Algorithm}

\subsection{Derivation}

Consider a transversal adaptive filter of order $M$, as depicled in Fig. 2. The output of this filter at time $n$ is

$$
y(n)=x^{\prime \prime}(n) w(n)
$$

where $\mathbf{x}(n)$ is an $M$-by- $I$ input vector at time $n$, i.e.,

$$
\mathrm{x}(n)^{T}=[x(n) x(n-1) \ldots x(n-M+1)]
$$

and $w(n)$ is the tap weights vector at that time, i.e.,

$$
w(n)^{T}=\left[w_{1}(n) w_{2}(n) \ldots w_{M}(n)\right]
$$

In this dissertation, bold face letters represent vector quantities. Scalars are in lower case italics. If $d(n)$ denotes the desired output at time $n$, then the error at this time is defined as

$$
e(n)=d(n)-y(n)
$$

In adjusting the tap wcights, the objective is to minimize the performance index which is defined as the expectation of the square of the error,

$$
\varepsilon(n)=E\left(|e(n)|^{2}\right)
$$


As stated before, the LMS algorithm is a recursive procedure to update the tap weights of the filter. At each time step $n+1$, the tap weights vector, $w(n+1)$, is calculated using the estimation error and the input vector at the previous time $n$. Therefore

$$
w(n+1)=w(n)+\mu x(n) c^{*}(n)
$$

The gradient step size, $\mu$, is a positive parameter limited to

$$
0<\mu<2 / \text { (total input power), }
$$

where

total input power $=\operatorname{Mr}(0)$.

$r(0)$ is the variance of input signal at time $n$, i.e.,

$$
r(0)=E\left[x(n) x^{*}(n)\right]
$$

Assuming an initial condition of $w(0)=0$, an iterative process can be used to compute the tap weights.

The conjugate of the estimated error is

$$
e^{\bullet}(n)=d^{*}(n)-w^{\prime \prime}(n) x(n)=d^{*}(n)-x^{\prime \prime}(n) w(n) .
$$

Substitute 4.4 into 4.3 to obtain

$$
\mathrm{w}(n+1)=\mathrm{w}(n)+\mu \mathrm{X}(n) d^{*}(n)-\mu \mathrm{X}(n) \mathrm{X}^{\prime \prime}(n) \mathrm{w}(n) .
$$

Definition 1: The difference vector for $s$ step update, $\Delta w(n+s)$, is defined as

$$
\Delta \mathrm{w}(n+s)=\mathrm{w}(n+s)-\mathrm{w}(n),
$$


Definition 2: The lookahead crror $p$ is defined as

$$
p(n+k)=\mathrm{x}^{\prime \prime}(n+k) \mathrm{w}(n)-d^{*}(n+k), \text { for } k \geq 0
$$

Definition 3: The weighted input vector $\hat{\mathbf{x}}(n+k)$ is defined as

$$
\hat{\mathbf{x}}(n+k)=-\mu \mathbf{x}(n+k) \text { for } k \geq 0 .
$$

Using 4.6, the difference vector for one step, $\Delta w(n+1)$, is

$$
\Delta w(n+1)=w(n+1)-w(n)
$$

Using equation 4.5 and the definitions of the weighted input vector and the lookahead error, we have

$$
\Delta w(n+1)=\hat{x}(n) p(n)
$$

Using 4.3 and 4.4 , and replacing $n$ with $n+1$ gives the weights for time $n+2$,

$$
\begin{aligned}
w(n+2) & =\mathrm{w}(n+1)+\mu \mathrm{x}(n+1) e^{*}(n+1), \\
& =\mathrm{w}(n+1)+\mu \mathrm{x}(n+1) d^{*}(n+1)-\mu \mathrm{x}(n+1) \mathrm{x}^{\prime \prime}(n+1) \mathrm{w}(n+1) .
\end{aligned}
$$

The difference vector for two step update, $\Delta w(n+2)$, is

$$
\Delta w(n+2)=w(n+2)-w(n) .
$$

Considering the definitions of $p$ and $\bar{x}, \Delta w(n+2)$ can be rewritten in terms of $x^{\prime} s, \hat{x}^{\prime} s$, and $p$ 's as

$$
\begin{aligned}
\Delta \mathrm{w}(n+2)= & \hat{\mathrm{x}}(n) p(n) \\
& +\hat{\mathrm{x}}(n+1) p(n+1) \\
& +\hat{\mathrm{x}}(n+1) \mathrm{x}^{\prime \prime}(n+1) \hat{\mathrm{x}}(n) p(n) .
\end{aligned}
$$


By continuing in the same manner, the general form of the difference vector for $s$ steps, $\Delta w(n+s)$, can be determined in terms of $\mathbf{x}$ 's, $\hat{x}$ 's, and $p$ 's. These terms are obtained from the following theorem.

Deflnition 4: $B_{r}$ is an ordered set of integer numbers which indicate the position(s) of 1 's in the binary presentation of any positive number $r$. Each $r$ is represented with $s$ bits and $2^{\prime}>r>0$. Elements of $B_{r}$ are ordered in descending value from left to right. For example $B_{4}=\{2\}, B_{6}=\{2,1\}, B_{8}=\{3\}, B_{11}=\{3,1,0\}$, and $B_{14}=\{3,2,1\} . B_{r}$ has at least one element, and at most $s$ elements. Then

$$
r=\sum_{i \in B_{r}} 2^{i}
$$

Definition 5: The least significant bit of a binary number with $s$ bits is the $0^{\text {th }}$ position and the right most bit.

Definition 6: The most significant bit of a binary number with $s$ bits is the $s-l^{a t}$ position and the left most bit.

$\checkmark$ Theorem: Interpreting all binary numbers with $s$ bits according to the following algorithm, generates all terms whose summation produces the value for the difference vector for $s$ slep update, $\Delta w(n+s)$.

Let $a_{r}=\min \left(B_{r}\right)$, the smallest member of $B_{r}$. Then $\Delta w(n+s)$ is generated as follows:

1. For all numbers, $r$, with $s$ bits:

(a) For the right most 1 of $r$, at $a_{r}^{\text {th }}$ position, assign $\bar{x}\left(n+a_{r}\right) p\left(n+a_{r}\right)$.

(b) For each other 1 at a $j^{\text {th }}$ position, $\left(j \in B_{r}-\left\{a_{r}\right\}\right)$, assign $\widehat{x}(n+j) \mathrm{x}^{H}(n+j)$. 
(c) Multiply all the assigned terms interpreted from 1's in the binary presentation

$$
\begin{aligned}
& \text { of } r \text { to produce a vector } v(r), \\
& v(r)= \begin{cases}\hat{\mathbf{x}}\left(n+a_{r}\right) p\left(n+a_{r}\right) & \text { if } r=2^{a_{r}} \\
{\left[\prod_{j \in B_{r}-\left\{a_{r}\right)} \hat{x}(n+j) x^{H}(n+j)\right] \hat{\mathbf{x}}\left(n+a_{r}\right) p\left(n+a_{r}\right)} & \text { otherwise }\end{cases}
\end{aligned}
$$

2. Add up all vectors, $v(r)$ 's, to calculate the value of difference vector for $s$ step update, $\Delta w(n+s)$,

$$
\Delta w(n+s)=\sum_{r=1}^{20-1} v(r)
$$

\section{PROOF:}

The proof is by induction.

For $s=1$ we have

$$
\begin{aligned}
\Delta w(n+1) & =\sum_{r=1}^{2^{\prime}-1} v(r) \\
& =v(1) \\
& =\hat{\mathbf{x}}(n) p(n),
\end{aligned}
$$

Which is directly derived in equation 4.9.

We show that if

$$
\Delta w(n+k)=\sum_{r=1}^{2^{k}-1} v(r),
$$

then

$$
\Delta w(n+k+1)=\sum_{r=1}^{2^{k+1}-1} v(r) .
$$

Using 4.3 we have

$$
\mathrm{w}(n+k+1)=\mathrm{w}(n+k)+\mu \mathrm{x}(n+k) e^{*}(n+k)
$$


where

$$
c(n+k)=d(n+k)-\mathbf{x}^{H}(n+k) \mathbf{w}(n+k)
$$

Therefore

$$
\mathrm{w}(n+k+1)=\mathrm{w}(n+k)+\mu \mathrm{x}(n+k)\left(d^{*}(n+k)-\mathrm{x}^{\prime \prime}(n+k) \mathrm{w}(n+k)\right)
$$

The difference vector is defined as

$$
\Delta \mathrm{w}(n+k+1)=\mathrm{w}(n+k+1)-\mathrm{w}(n)
$$

Using 4.10 and 4.11 and definition of lookahend crrors, p's, we write

$$
\begin{aligned}
\Delta w(n+k+1) & =\Delta w(n+k)+\hat{x}(n+k) p(n+k)+\hat{x}(n+k) x^{\prime \prime}(n+k) \Delta w(n+k) \\
& =\sum_{r=1}^{2^{k}-1} v(r)+v\left(2^{k}\right)+\hat{x}(n+k) x^{\prime \prime}(n+k) \sum_{r=1}^{2^{k}-1} v(r) \\
& =\sum_{r=1}^{2^{k}-1} v(r)+v\left(2^{k}\right)+\hat{x}(n+k) x^{\prime \prime}(n+k) \\
& =\sum_{r=1}^{2^{k}-1} v(r)+\sum_{r=1}^{2^{k}}\left(\prod_{j \in B_{r}-a_{r}} \hat{\mathbf{x}}(n+j) \mathbf{x}^{\prime \prime}(n+j)\right) \hat{x}\left(n+a_{r}\right) p\left(n+a_{r}\right) \\
& \left.\hat{\mathbf{x}}_{(n+j)}(n) x^{\prime \prime}(n+j)\right) \hat{x}\left(n+a_{r}\right) p\left(n+a_{r}\right) \\
& =\sum_{r=1}^{2^{k}} v(r)+\sum_{r=2^{k}+1}^{2^{k+1}-1} v(r) \\
& =\sum_{r=1}^{2^{k+1}-1} v(r) .
\end{aligned}
$$

8

As an example, the terms of the difference vector for up to $s=4$ are derived from the bit string representation as shown in Table 3. This figure shows how each 1 of binary 
representation of number $r$, with $s$ bits, at the $r^{\text {th }}$ row is translated into a proper term in order to form the vector $v(r)$. Each $v(r)$ is expressed in terms of the input vectors, $x$ 's, the weighted input vectors, $\hat{\mathbf{x}}$ 's, the lookahead errors, $p$ 's, all from time $n$ up to time $n+s-1$, and tap weights at time $n, w(n)$.

Table 3: Terms of $\Delta w(n+4)$

\begin{tabular}{|c|c|c|c|c|c|c|c|c|c|}
\hline$\frac{R_{m}}{r}$ & $\frac{\mathrm{Bim}}{3}$ & $\frac{x^{2}}{2}$ & $\frac{x+m}{1}$ & & $\mathrm{~m}+3$ & 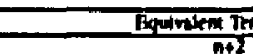 & n4 & & thent \\
\hline 1 & 0 & 0 & $n$ & 1 & & & & $\overline{\mathbf{X}}_{(n) n(n)}$ & $\mathbf{v}(1)$ \\
\hline 2 & 0 & $n$ & 1 & $n$ & & & $\hat{\mathbf{x}}(n+1) m(n+1)$ & & $V(z)$ \\
\hline 3 & 0 & $n$ & 1 & $\mathbf{I}$ & & & $\overline{\mathbf{x}}(n+1) \mathbf{X}^{\prime \prime}(n+1)$ & $\hat{X}(n) M(n)$ & $v_{(3)}$ \\
\hline 4 & 0 & I & 0 & $n$ & & $\overline{\mathbf{x}}(n+2) n(n+2)$ & & & $V(0)$ \\
\hline 3 & " & 1 & " & 1 & & $\hat{x}(n+2) X^{\prime \prime}(n+2)$ & & $\bar{X}(n) \mu(n)$ & $V(s)$ \\
\hline 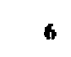 & 0 & I & t & o & & $\hat{\mathbf{X}}(n+2) \mathbf{X}^{\mu}(n+2)$ & $\hat{\mathbf{X}}_{(n+1) n(n+1)}$ & & $V(n)$ \\
\hline 7 & n & 1 & 1 & 1 & & $\hat{\mathbf{x}}(n+2) X^{\prime \prime}(n+2)$ & $\hat{x}_{(n+1)} X^{\prime \prime}(n+1)$ & $\hat{\mathbf{x}}(n)+(n)$ & $v(\eta)$ \\
\hline 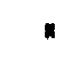 & 1 & " & n & 0 & $\hat{\mathbf{x}}(n+3) R^{(n+3)}$ & & & & $V(1)$ \\
\hline 9 & 1 & " & " & 1 & $\hat{X}_{(n+3)} X^{\prime \prime}(n+3)$ & & & $\hat{X}(n) n(n)$ & $V(0)$ \\
\hline 10 & 1 & 0 & 1 & o & $\hat{\mathbf{X}}_{(n+3)} \mathbf{X}^{\prime \prime}(n+3)$ & & $\hat{x}_{(n+1) n(n+1)}$ & & $\mathbf{V}(10)$ \\
\hline 11 & 1 & $n$ & 1 & 1 & $\hat{\mathbf{x}}(n+3) X^{\prime \prime}(n+3)$ & & $\hat{\mathbf{X}}_{(n+1)} \mathbf{X}^{n}(n+1)$ & $\hat{\mathbf{X}}_{(n) r(n)}$ & $V_{(11)}$ \\
\hline 12 & 1 & 1 & " & n & $\hat{\mathbf{X}}_{(n+3) X^{\prime \prime}(n+3)}$ & $\overline{\mathbf{X}}(n+2) p(n+2)$ & & & $v(12)$ \\
\hline 13 & 1 & 1 & n & 1 & $\hat{x}_{(n+3) x^{\prime \prime}(n+3)}$ & $\hat{\mathbf{x}}_{(n+2)} \mathbf{X}^{\prime \prime}(n+2)$ & & $\hat{\mathbf{x}}_{(n) n(n)}$ & $v_{(13)}$ \\
\hline 14 & 1 & 1 & 1 & 0 & $\overline{\mathbf{X}}_{(n+3)} \mathbf{X}_{(n+3)}$ & $\hat{x}_{(n+2)} X^{\prime \prime}(n+2)$ & $\overline{\mathbf{x}}(n+1) n(n+1)$ & & $v(14)$ \\
\hline is & 1 & 1 & 1 & 1 & $\hat{\mathbf{X}}_{(n+3)} \mathbf{X}^{\prime \prime}(n+3)$ & $\overline{\mathbf{X}}_{(n+2)} X^{\prime \prime}(n+2)$ & $\bar{x}(n+1) X^{\prime \prime}(n+1)$ & $\hat{X}(n) N(n)$ & $V_{(19)}$ \\
\hline
\end{tabular}

Notice that for each row $r$, whose binary presentation has " 1 " in $m$ positions, $v(r)$ is the product of $m-1$ autocorrelation matrices, $\hat{x}(i) x^{H}(i)$, multiplied by the most right vector, $\hat{\mathbf{x}}(k) p(k)$. Also, notice that, each $\mathbf{x}^{\prime \prime}(i) \hat{\mathbf{x}}(j), i \neq j$, term is the inner product of two vectors, resulting a scalar value. Therefore, $v(r)$ is the product of $m+1$ scalars multiplied by the most left vector $\hat{x}(j)$. This partitioning of the terms in each row leads to the following definitions and algorithm. 
Definition 7: The scalar $c$ is defined as

$$
c(i, j)=\mathrm{x}^{\prime \prime}(n+i) \hat{\mathbf{x}}(n+j) ; \text { for } i=1, \ldots, s-1, \text { and } j=0,1, \ldots, i-1 \text {. }
$$

Definition 8: For any positive number $r, C_{r}$ is the set of all element pairs formed from all adjacent elements of $B_{r}$ when $B_{r}$ has more than one element, $\left\|B_{r}\right\|>1 . C_{r}=\phi$ if $B_{r}$ has only one clement, $\left\|B_{\mathrm{r}}\right\|=1$. For example $C_{6}=\{(2,1)\}, C_{8}=\phi, C_{11}=\{(3,1),(1,0)\}$, and $C_{14}=\{(3,2),(2,1)\}$.

These definitions allow simplification of the terms of $\Delta w(n+s)$. The following algorithm expresses the difference vector for $s$ step update, $\Delta w(n+s)$, using $\hat{x} ' s, p$ 's, and c's:

Algorithm: Let $a_{\mathrm{r}}$ and $b_{\mathrm{r}}$ be the smallest and the largest elements of the $B_{\mathrm{r}}$, i.c., $a_{\mathrm{r}}=$ $\min \left(B_{r}\right)$ and $b_{r}=\max \left(B_{r}\right)$. Then

1. For all numbers $r$ with $s$ bits:

(a) Assign $p\left(n+a_{r}\right)$ for the right most 1 in the number at $a_{r}^{\text {th }}$ position.

(b) Assign $\hat{x}\left(n+b_{r}\right)$ for the left most 1 in the number at $b_{r}^{\text {th }}$ position.

(c) If $C_{\mathrm{r}} \neq \phi$ then multiply $\hat{\mathbf{x}}\left(n+b_{r}\right), p\left(n+a_{\mathrm{r}}\right)$, and all $c(i, j)$ terms for each element of $C_{r}$ to calculate the vector $v(r)$. If $C_{r}=\phi$ then $\operatorname{set} v(r)=\hat{\mathbf{x}}\left(n+a_{r}\right) p\left(n+a_{r}\right)$. That is

$$
v(r)= \begin{cases}\hat{x}\left(n+a_{r}\right) p\left(n+a_{r}\right) & \text { if } r=2^{a_{r}} \\ \hat{x}\left(n+b_{r}\right)\left[\prod_{(i, j) \in C_{r}} c(i, j)\right] p\left(n+a_{r}\right) & \text { otherwise }\end{cases}
$$

2. Add all $v(r)$ for $r=1$ to $r=2^{4}-1$ to obtain the difference vector $\Delta w(n+s)$,

$$
\Delta w(n+s)=\sum_{r=1}^{20-1} v(r) .
$$


Table 4: $\Delta w(n+4)$ in terms of $\hat{\mathbf{x}}, p$, and $c$

\begin{tabular}{|c|c|c|c|c|c|}
\hline Row & \multicolumn{3}{|c|}{ Binary Presentation } & \multirow[t]{2}{*}{ Equivalent Terms } & \multirow[b]{2}{*}{ Vectors } \\
\hline $\mathbf{r}$ & 32 & 1 & $\overline{0}$ & & \\
\hline 1 & 00 & 0 & 1 & $\hat{\mathbf{x}}(n) p(n)$ & $v(1)$ \\
\hline 2 & 00 & 1 & 0 & $\hat{\mathbf{x}}(n+1) p(n+1)$ & $v(2)$ \\
\hline 3 & 00 & 1 & 1 & $\hat{\mathbf{x}}(n+1) c(1,0) p(n)$ & $v(3)$ \\
\hline 4 & 01 & 0 & 0 & $\hat{\mathbf{x}}(n+2) p(n+2)$ & $v(4)$ \\
\hline 5 & 01 & 0 & 1 & $\hat{\mathbf{x}}(n+2) c(2,0) p(n)$ & $v(5)$ \\
\hline 6 & 01 & 1 & 0 & $\hat{\mathbf{x}}(n+2) c(2,1) p(n+1)$ & $v(6)$ \\
\hline 7 & 01 & 1 & 1 & $\hat{\mathbf{x}}(n+2) c(2,1) c(1,0) p(n)$ & $v(7)$ \\
\hline 8 & 10 & 0 & 0 & $\overline{\mathbf{x}}(n+3) p(n+3)$ & $v(8)$ \\
\hline 9 & 10 & 0 & 1 & $\hat{\mathbf{x}}(n+3) c(3,0) p(n)$ & $v(9)$ \\
\hline 10 & 10 & 1 & 0 & $\hat{\mathbf{x}}(n+3) c(3,1) p(n+1)$ & $v(10)$ \\
\hline 11 & 10 & 1 & 1 & $\hat{\mathbf{x}}(n+3) c(3,1) c(1,0) p(n)$ & $v(11)$ \\
\hline 12 & 11 & 0 & 0 & $\hat{\mathbf{x}}(n+3) c(3,2) p(n+2)$ & $v(12)$ \\
\hline 13 & 1 & 0 & 1 & $\hat{\mathbf{x}}(n+3) c(3,2) c(2,0) p(n)$ & $v(13)$ \\
\hline 14 & 11 & 1 & 0 & $\hat{\mathbf{x}}(n+3) c(3,2) c(2,1) p(n+1)$ & $v(14)$ \\
\hline 15 & 11 & 1 & 1 & $\hat{\mathbf{x}}(n+3) c(3,2) c(2,1) c(1,0) p(n)$ & $v(15)$ \\
\hline
\end{tabular}

Table 4 shows the terms of row vectors of the difference vector for 4 step update, $\Delta w(n+4)$, in terms of $\hat{x}^{\prime} s, c^{\prime} s$, and $p$ 's. Figure 6 presents the block diagram of an architecture to support calculation of the new weights. To achicve minimum time in updating the tap weights of the adaptive filter, the blocks of Figure 6 are implemented using parallel techniques.

In the $X$ block, $s$ new $\hat{x}$ 's are calculated as

$$
\widehat{x}(n+i)=-\mu x(n+i) ; \text { for } i=0,1, \ldots, s-1 \text {. }
$$




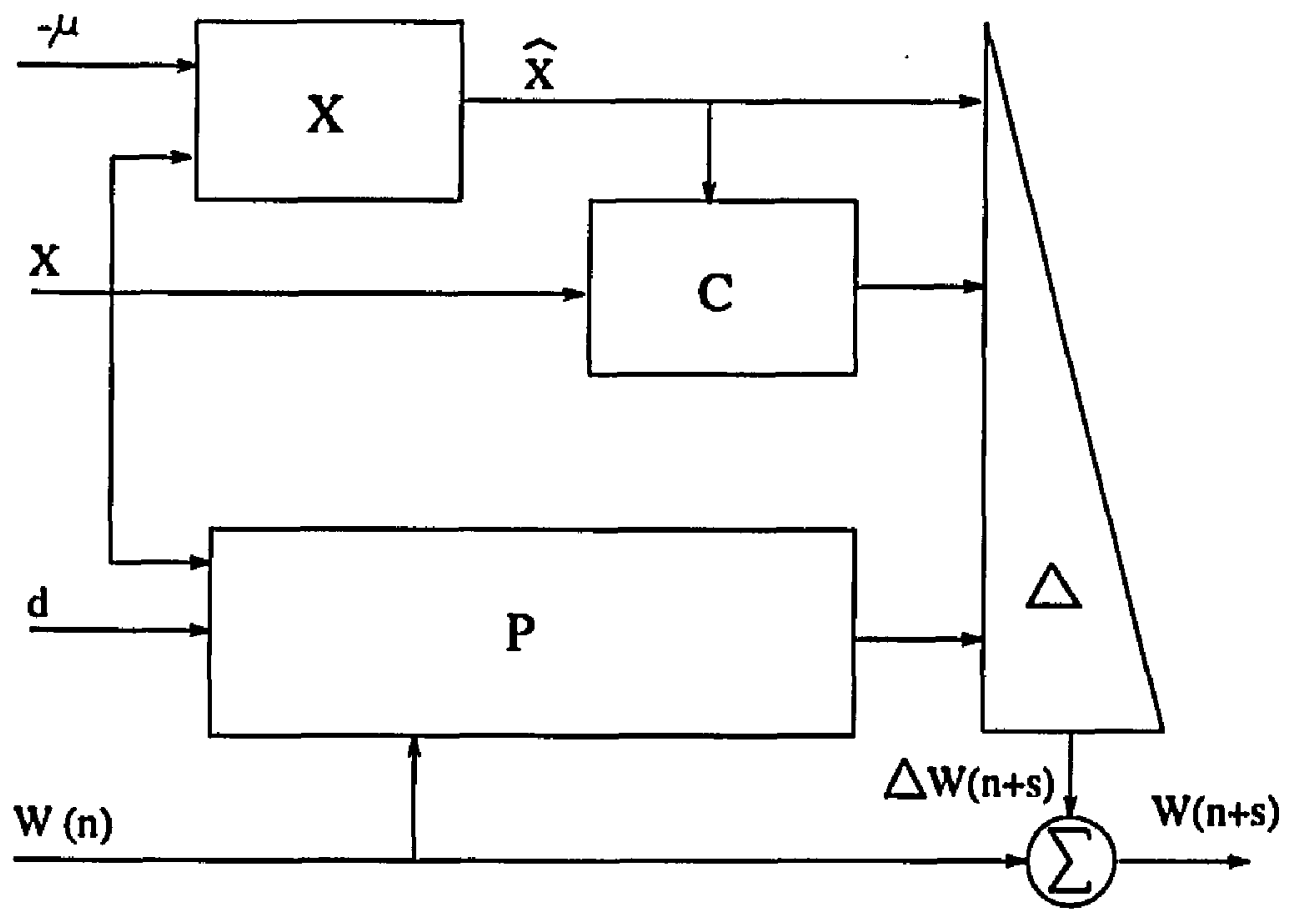

Figure 6: Block Diagram of the Adaptive Filter using Binary algorithm

In the $C$ block, the $\frac{(0-1)}{2} c$ 's are computed as mentioned in equation 4.12 . In the $P$ block, $s$ new $p$ 's are calculated as

$$
p(n+k)=\mathrm{x}^{\prime \prime}(n+k) \mathrm{w}(n)-d^{\prime \prime}(n+k), \text { for } k=0,1, \ldots, s-1 .
$$

In the $\Delta$ block, $x$ 's, $c$ 's, and $p$ 's are used to calculate the difference vector $\Delta w(n+s)$. Adding $\Delta w(n+s)$ to $w(n)$ in the last stage produces the tap weights of time $n+s, w(n+s)$. The following example clarifies the function of each block for an adaptive filter.

\subsection{Example}

As an example, consider an adaptive filter of order $8, M=8$. It is desired to construct a circuit for four step update, $s=4$. 
In the $X$ block the $\widehat{x}(n+i)$ terms are calculated. For this example, the $X$ block computes

$$
\widehat{x}(n+i)=-\mu x(n+i) ; \text { for } i=0,1,2,3 .
$$

$M-1$ old $x$ 's, i.e., $\hat{x}(n+i)$ for $i=-7,-6,-5,-4,-3,-2,-1$, are used from the previous block step.

In the $C$ block, the six $c$ coefficients are computed. They are:

$$
\begin{aligned}
& c(3,2)=x^{\prime \prime}(n+3) \hat{x}(n+2) \\
& c(3,1)=x^{\prime \prime}(n+3) \hat{x}(n+1) \\
& c(3,0)=x^{\prime \prime}(n+3) \hat{x}(n) \\
& c(2,1)=x^{\prime \prime}(n+2) \hat{x}(n+1) \\
& c(2,0)=x^{\prime \prime}(n+2) \hat{x}(n) \\
& c(1,0)=x^{\prime \prime}(n+1) \hat{x}(n)
\end{aligned}
$$

In the $P$ block four $p$ 's are determined as

$$
\begin{aligned}
& p(n+3)=\mathrm{x}^{\prime \prime}(n+3) \mathrm{w}(n)-d^{*}(n+3) \\
& p(n+2)=\mathrm{x}^{\prime \prime}(n+2) \mathrm{w}(n)-d^{*}(n+2) \\
& p(n+1)=\mathrm{x}^{\prime \prime}(n+1) \mathrm{w}(n)-d^{*}(n+1) \\
& p(n)=\mathrm{x}^{\prime \prime}(n) \mathrm{w}(n)-d^{*}(n)
\end{aligned}
$$

Within the $\Delta$ block the outputs of the $X, C$ and $P$ blocks are multiplied and summed to produce $\Delta w(n+s)$. The exact multiplications and additions to be performed are presented in table 4 . 


\subsection{Convergence}

In order to test the convergence of the Parallel Binary Structure LMS algorithm, an unknown system identification is considered. The unknown system under consideration is a FIR filter of order 20. An adaptive filter of order $M=16$ estimates the unknown system.

The convergence of PBS LMS is evaluated in the two cases. In the first case, the gradient step size is constant and the eigenvalue spread of the input correlation matrix is variable. In the second case, the gradient step size is variable and the eigenvalue spread is constant.

In order to test the Parallel Binary Structure LMS a random process with variable eigenvalue spread is needed. To produce such a random process, a random variable $u(k)$ is applied to an Auto Regressive (AR) filter. $u(k)$ is a Gqussian white noise random variable with zero mean and variance of $\sigma_{\mathrm{u}}$. The AR filter has a transfer function in the form of

$$
H(z)=\frac{1}{1+a z^{-1}+b z^{-2}}
$$

The output of the AR filter is a zero mean random process (RP), $x(k)$. The variance of the random process, $\sigma_{x}$, is

$$
\sigma_{x}=\rho \sigma_{u}
$$

where

$$
\rho=\left((1+b)^{2}-a^{2}\right) \frac{1+b}{1-b} .
$$

In order to have a zero mean, unity variance RP, the variance of the white noise $u(k)$ is selected to be

$$
\sigma_{u}=\frac{1}{\rho} \text {. }
$$


The $2 \times 2$ correlation matrix of the above filter has two cigenvalues. These cigenvalues are

$$
\lambda_{\max }=\left(1+\frac{a}{1+b}\right) \sigma_{x}^{2}
$$

and

$$
\lambda_{\min }=\left(1-\frac{a}{1+b}\right) \sigma_{x}^{2}
$$

The eigenvalue spread of the correlation matrix is defined as

$$
\Lambda=\frac{\lambda_{\max }}{\lambda_{\min }}
$$

which is a function of filter variables $a$ and $b$. Therefore, by controlling the values of $a$ and $b$ in the above filter, different eigenvalue spreads for random processes are obtained.

In the first case, the gradient step size is kept constant, $\mu=0.005$. With constant value for $b$ coefficient in the AR filter, three values for $a$ are calculated in order to have three different and distinguished values for eigenvalue spread, $\Lambda=100,50$, and 5 .

Then at each iteration, a Gaussian white noise with variance of $\sigma_{u}=\frac{1}{\rho}$ is applied to the AR filter. $\rho$ is calculated using Eq. 4.14. The outputs of the AR filter, in turn, are applied to the unknown system. The outputs of the unknown system are the desired outputs for the adaptive filter. The outputs of the AR filter are the inputs of the adaptive filter. The adaptive filter uses the PBS LMS algorithm to adjust its tap weights in such a way that the square of the error between the real output and the desired output is minimized.

Repeating the above procedure for 40 times, the average of the square of the errors for each time instance is calculated. 
The effect of the different eigenvalue spreads on the convergence of the PBS LMS is shown in Fig. 7. To obtain each curve of this figure, an adaptive filter of order $M=16$ is considered to estimate the unknown system. The unknown system is an FIR filter of order 20. In the parallel algorithm the parallel step size is kept constant $s=8$. Fig. 7 shows the PBS $\perp M S$ algorithm has perfect LMS convergence.

In the second part the eigenvalues spread of the correlation matrix is constant and equal to 50. Then the effect of three gradient step sizes $\mu=0.005,0.001$, and 0.0005 are investigated. This simulation, on an adaptive filter of order 16 and the parallel step size 8 , is repeated for 40 iterations. The result of these simulations are shown in Fig. 8.

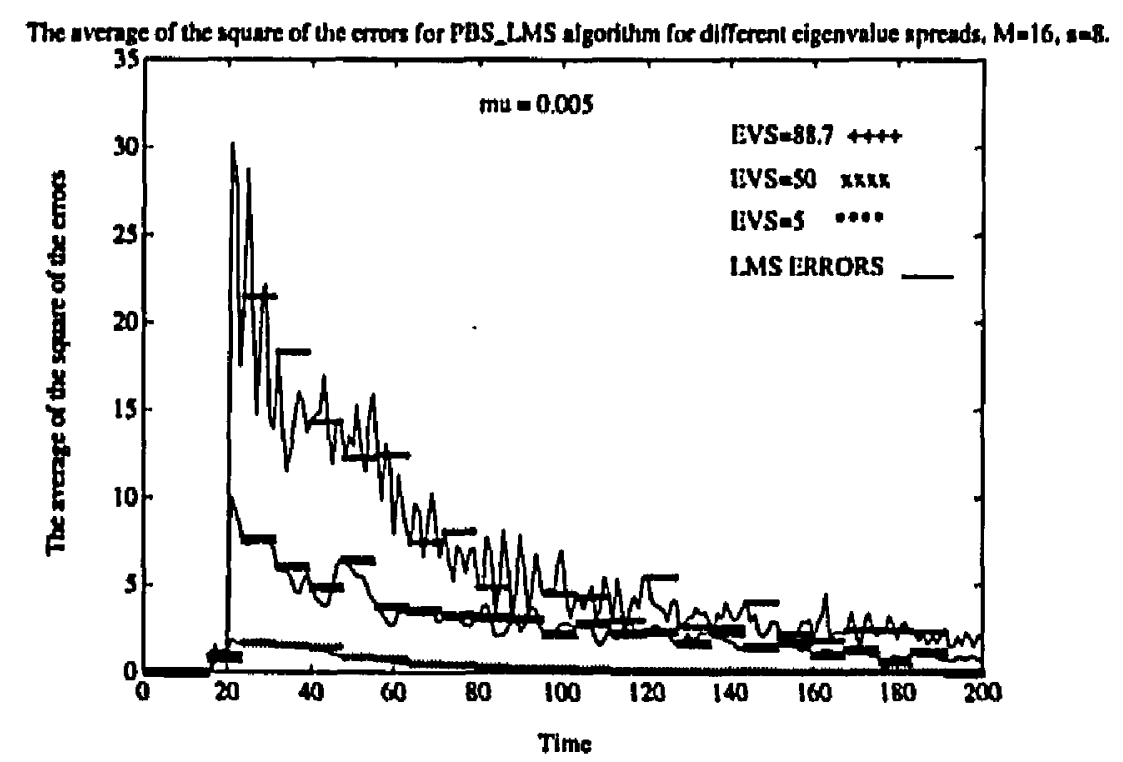

Figure 7: Convergence of the PBS $\perp M S$ algorithm for different eigenvalue spread, when gradient step size is constant $\mu=0.005, M=16, s=8$

In the Fig. 7 and Fig. 8 the average of the square of the errors of an adaptive filter based on the original LMS is also depicted. These figures show that the MSE curves of 


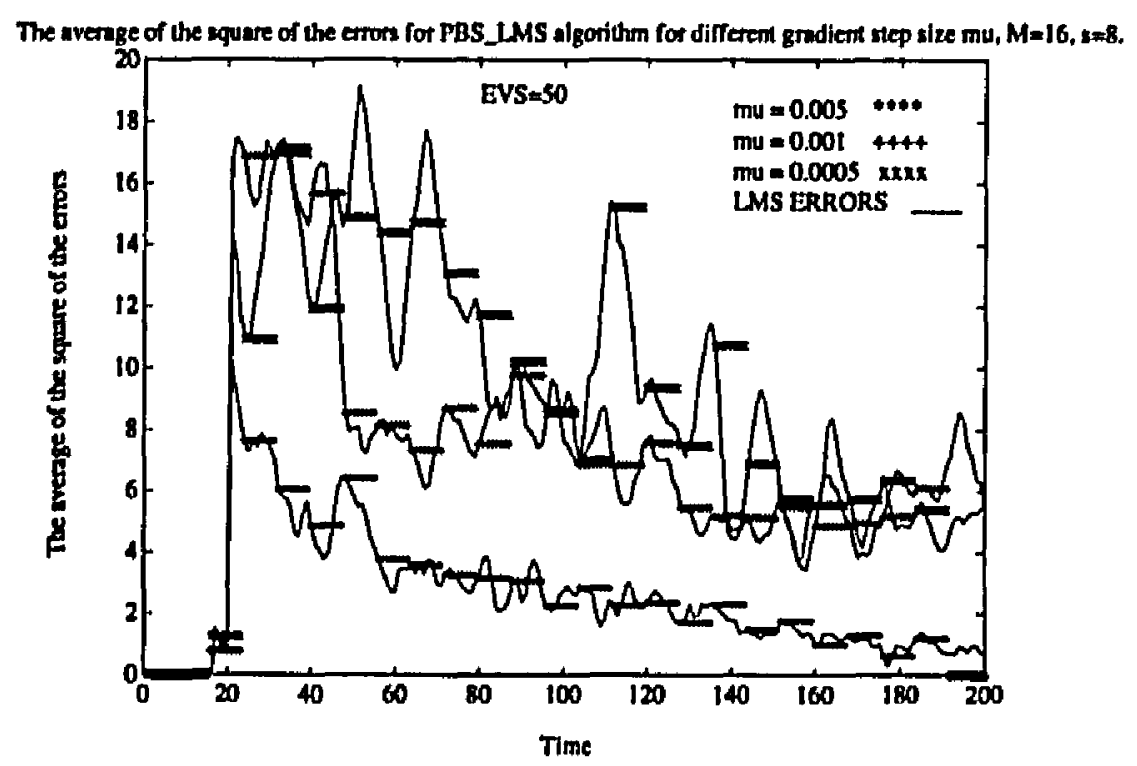

Figure 8: Convergence of the PBS $\perp M S$ algorithm for different gradient step size, when cigenvalue spread is constant $\Lambda=50, M=16, s=8$

the PBS_LMS algorithm, with $s$ step jumps, traces exactly the MSE curve of the original LMS algorithm. This similarity of PBS.LMS and LMS algorithms is a big advantage for the PBS_LMS algorithm. Based on this exact performance, one can apply all the proposed methods for improving the convergence speed of the sequential LMS to the PBS LMS.

The result of another simulation of two adaptive filters, one based on PBS LMS and the other based on the original LMS algorithms, is shown in Fig. 9. In this simulation the gradient step size and the eigenvalue spread of the random processes are constant, $\mu=0.005, \Lambda=50$. This simulation is run for 500 samples and the square of the errors were averaged over 50 trials. Fig. 9 shows the average of the square of the errors of two adaptive filters for all samples. As Fig. 9 shows, the average of the errors of both filters after first 200 samples are identical. For better distinction, Fig. 10 depicts the average of 
the square of the errors for the first 200 samples. The average of the errors of the filters for samples after 200 is shown in Fig. 11.

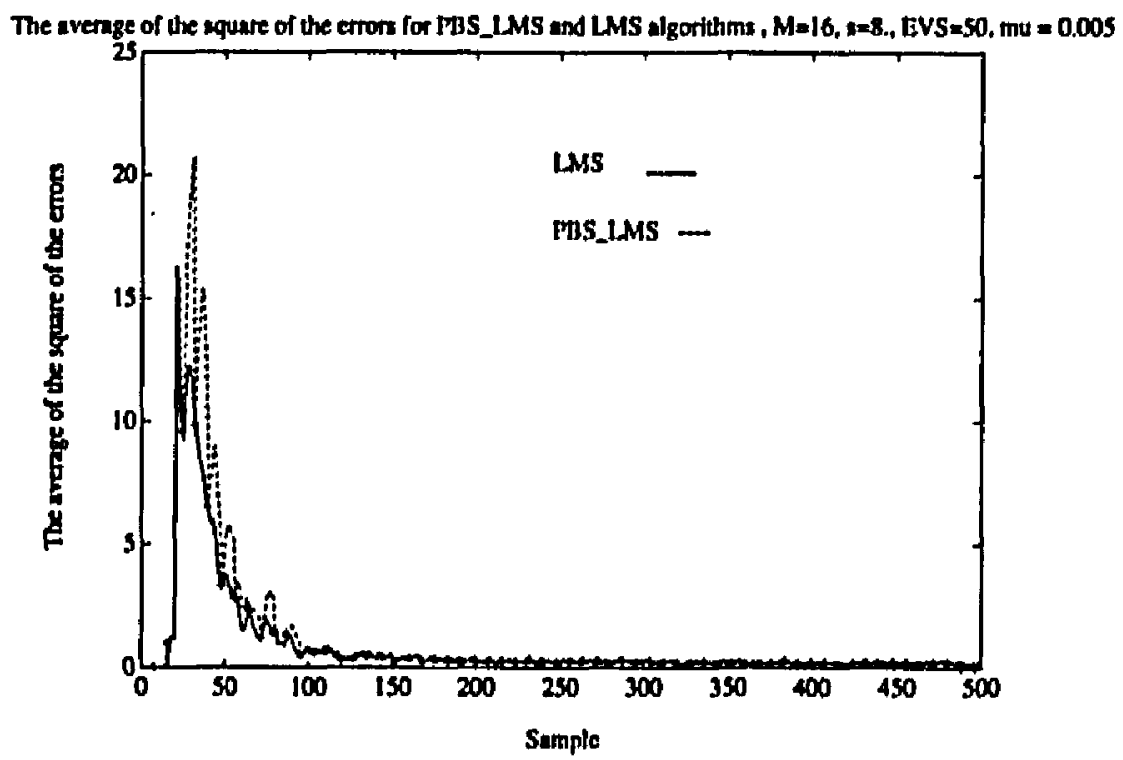

Figure 9: The average of square of the errors of two adaptive filters based on PBS.LMS and original LMS algorithms

The outputs of the adaptive filters using PBS.LMS and original LMS algorithms for a constant gradient step size, $\mu=0.005$, and constant eigenvalue spread, $\Lambda=50$, is shown in Fig. 12. The normalized difference between the outputs of the PBS.LMS and the original LMS based adaptive filters, $\frac{\text { output(LMS)-output(PBS LMS) }}{\text { oulput(LMS) }}$, is depicted in Fig. 13. These two figures, Fig. 12 and Fig. 13, are based on an experiment receiving 500 samples and show that both filters have identical outputs after convergence. The average of the difference between the outputs of the two filters is less than $0.1 \%$. 


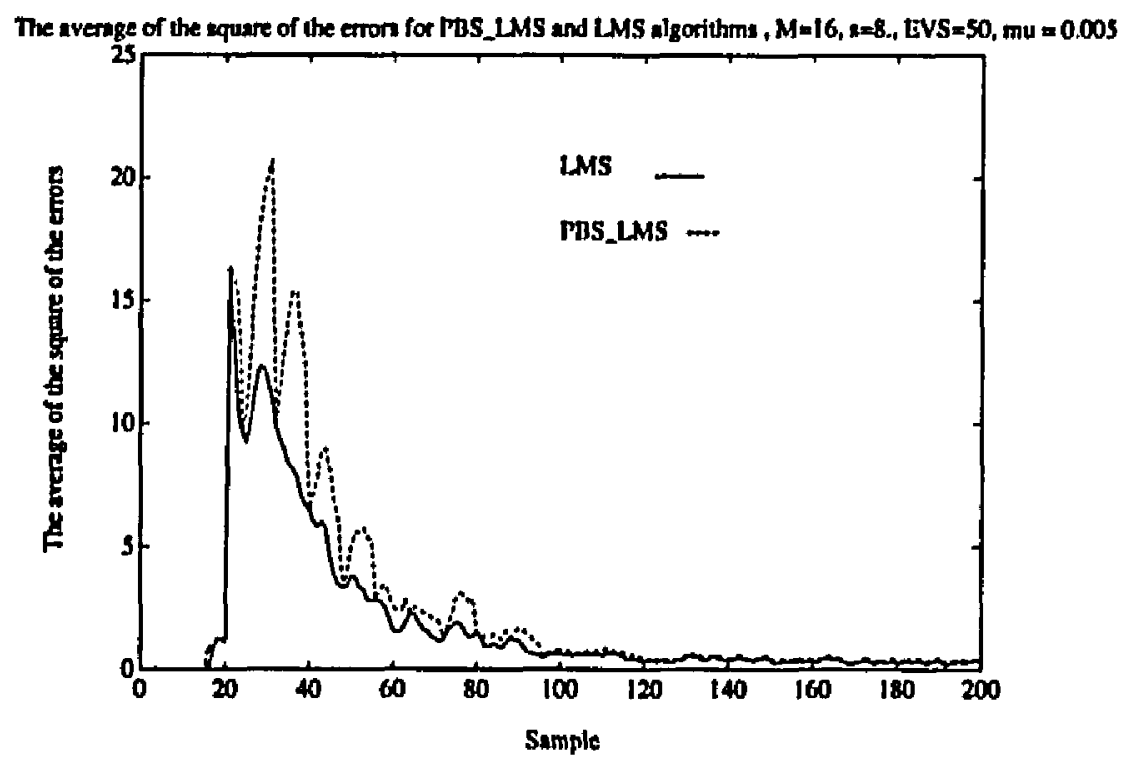

Figure 10: The average of square of the errors of two adaptive filters based on PBS LMS and original LMS algorithms for the first 200 samples

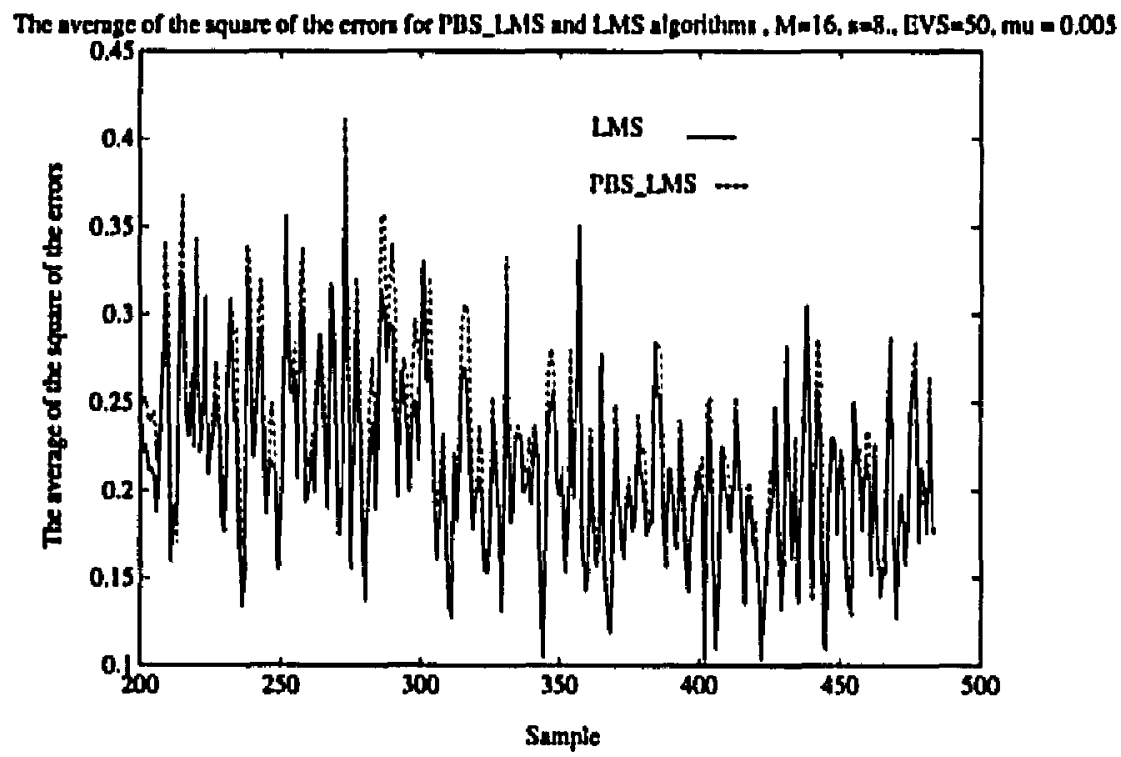

Figure 11: The average of square of the errors of two adaptive filters based on PBS_LMS and original LMS algorithms for samples over 200 


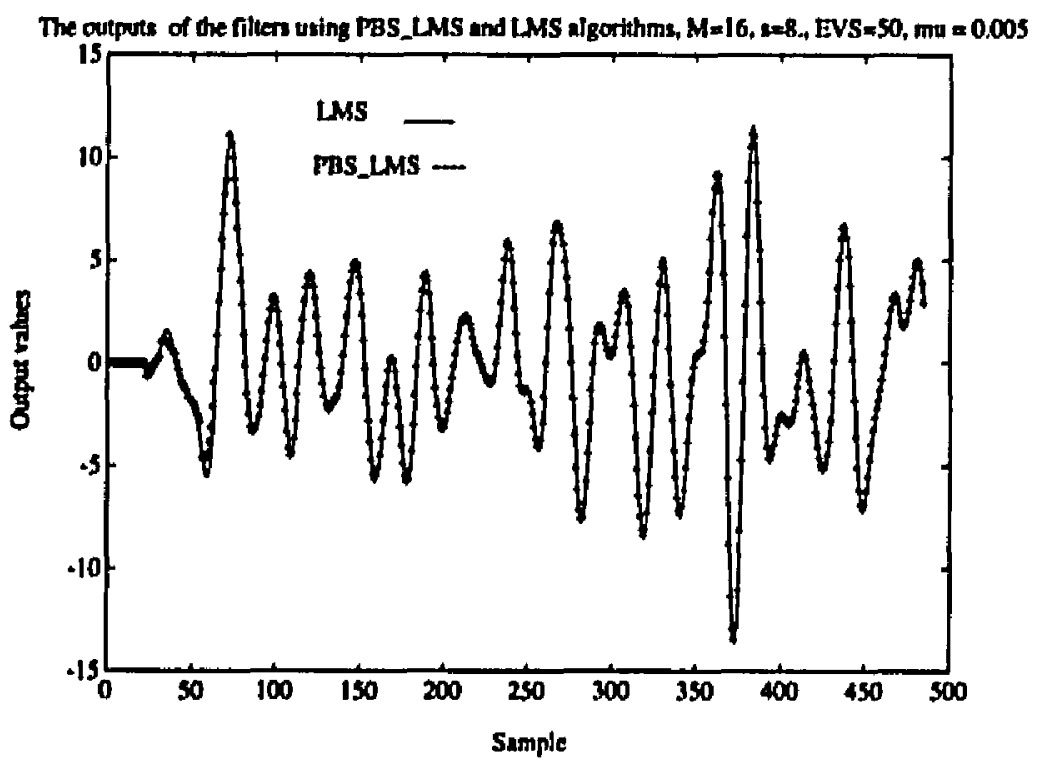

Figure 12: The outputs of two adaptive fillers based on PBS LMS and original LMS algorithms

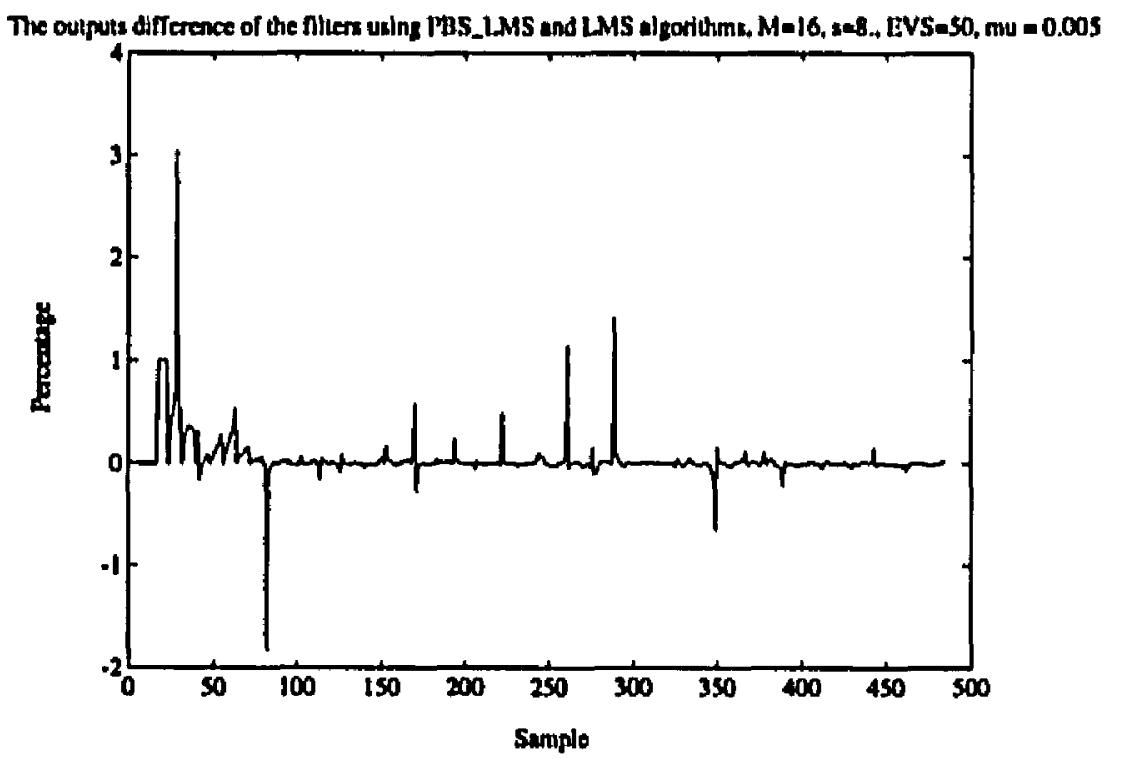

Figure 13: The difference between the outputs of two adaptive filters based on PBS.LMS and original L.MS algorithms 


\section{CHAPTER V}

\section{Architectural Tradeoff of Parallel Binary Structured LMS Algorithm Blocks}

The binary algorithm presented is both regular and modular. The $\mathrm{X}, \mathrm{C}$, and $\mathrm{P}$ blocks have simple operations to perform. They prepare $\hat{x}, c$, and $p$ variables to be used in the $\Delta$ block. The $\Delta$ block has a binary numbered structure and can be built for any values of $M$ and $s$.

The computations within each block can be performed sequentially or in parallel. Additionally the computation of $\mathrm{X}$ and $\mathrm{C}$ can be performed in parallel with the computation of $P$. The maximum speedup occurs when the operations are performed with the maximum degree of parallelism. However, using the maximum degree of parallelism increases the number of computational units required. In this section the number of computational units required as well as the time delay of each block are evaluated.

\subsection{Evaluation of the $\mathrm{X}$ block:}

In the first stage, the product of $(-\mu)$ and $x$ gives the weighted values of of the input vectors, $\hat{\mathbf{x}}_{,}$as stated in equation 4.8. These vectors contain inputs from $x(n)$ to $x(n+s-1)$. If $\mu$ is a negative power of 2, i.e., $\mu=2^{-\nu}$ for a positive integer $\nu$, then the $X$ block performs only a shift operation, instead of a multiplication. 


\subsection{Evaluation of the $\mathbf{C}$ block:}

In the $C$ block, the scalar values of the $c$ 's are computed as given in equation 4.12. There arc $\frac{s(s-1)}{2} c$ coefficients required to form $\Delta w(n+s)$. Each $c$ coefficient is the result of a dot product.

One approach is to calculate the $c$ coefficients direclly from the input values, without using any of the values of the $c$ 's from the previous input block. The terms that need to be computed are shown in figure 14. In this figure, cach circle represents a multiplication, and each parallelogram outlines the products that need to be summed for each $c(i, j)$. There are a total of $\frac{(2 M+a-2)(s-1)}{2}$ multiplications. These multiplications are independent and may be done in parallel. Each of the $c$ coefficients then requires summation of $M$ terms requiring $M-1$ additions for a total of $\frac{s(s-1)(M-1)}{2}$ additions. Performing all multiplications in parallel and using parallel adder trees, the minimum time of execution for the $\mathbf{C}$ block is

$$
T_{C 1}=T_{\text {mult }}+\left[\log _{2} M\right\rceil T_{\text {add }}
$$

where $T_{\text {mult }}$ is the time to complete a multiplication operation and $T_{\text {add }}$ is the time to add two numbers. This maximally parallel architecture is refered to as $\mathrm{Cl}$.

When $M$ is sufficiently larger than $s$, which is the case in the most practical applications, then the $c$ 's can be calculated using the values of c's from the previous input block. Based on this approach, the $\mathrm{C}$ block can be built using different architectures. Figure 15 shows the relationship of the $c$ 's needed for two succesive calculations of $\Delta w(n)$.

One approach, we call it $\mathrm{C2}$, is to add-to and subtract-from each $c$ of the previous input 


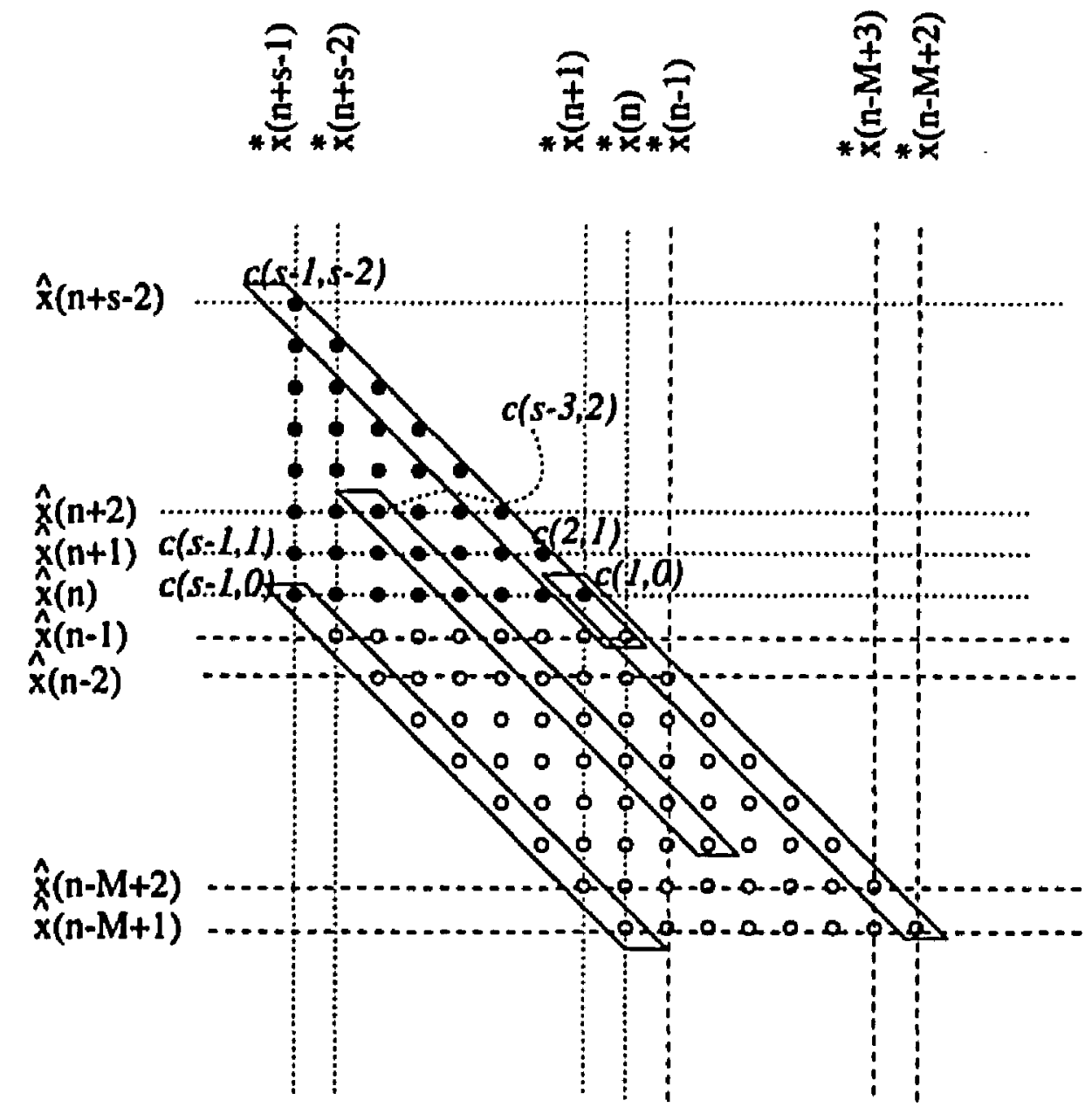

Figure 14: $\mathrm{C}$ block configuration for direct calculation, $\mathrm{Cl}$ method

blocks to obtain the new value for that $c$ coefficient. That is

$$
\begin{aligned}
c_{\text {new }}(i, j)= & c_{\text {old }}(i, j)+\sum_{k=0}^{-1} x^{*}(n+i-k) \hat{x}(n+j-k)- \\
& \sum_{k=0}^{-1} x^{*}(n+i-k-M) \hat{x}(n+j-k-M) \\
& \text { for } i=1, \ldots, s-1, \text { and } j=0,1, \cdots, i-1 .
\end{aligned}
$$

Assume

$$
x-h i s t o r y(i, j)=\sum_{k=0}^{a-1} x^{*}(n+i-k-M) \hat{x}(n+j-k-M),
$$



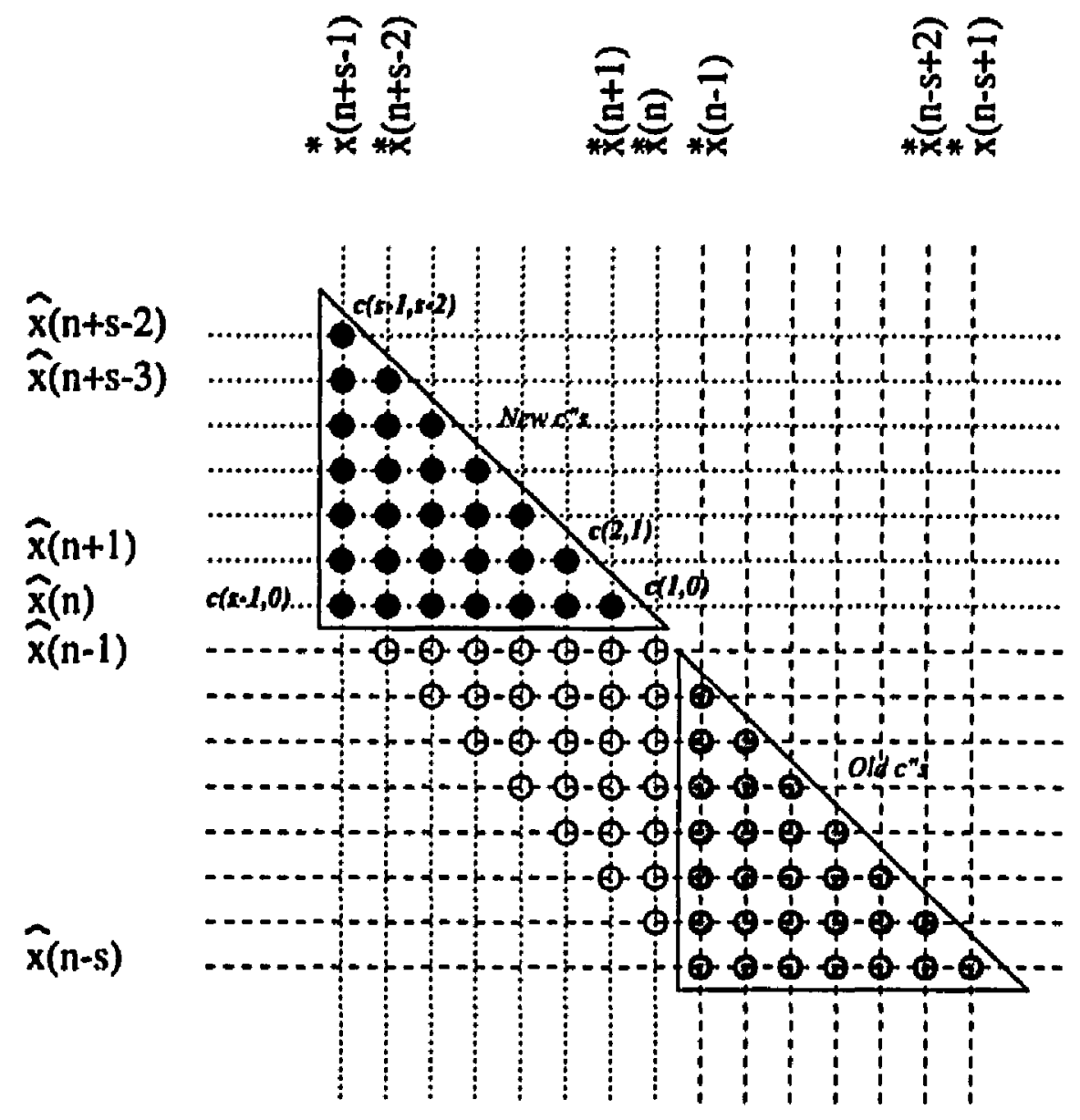

Figure 15: C block configuration for recursive calculation

then 5.1 can be written as

$$
\begin{aligned}
c_{\text {new }}(i, j)= & c_{\text {old }}(i, j)+\sum_{k=0}^{p-1} x^{*}(n+i-k) \hat{x}(n+j-k)-x_{-} \text {history }(i, j) . \\
& \text { for } i=1, \ldots, s-1, \text { and } j=0,1, \cdots, i-1 .
\end{aligned}
$$

Figure 16 shows the block diagram of $\mathrm{C} 2$ architecture for the $\mathrm{C}$ block. In this method, the results of the multipliers on the upper triangle is saved in the registers of the lower triangle. The products of the new inputs, calculated in the multipliers, are the inputs to the s_number adders. The results of these s_number adders are saved in the $x$ _history shift registers. 
These summations also are added to the corresponding $C_{\text {old }}-x$ Jistory to obtain the new values of the $c$ 's, as stated in Eq. 5.3. Note that the output of the $x$.history are the outputs of the s.number adders, which are calculated $\frac{M}{m}$ blocks prior. This architecture requires $s(s-1)$ multiplications and $s^{2}(s-1)$ additions. The minimum time of calculating new $c$ 's is

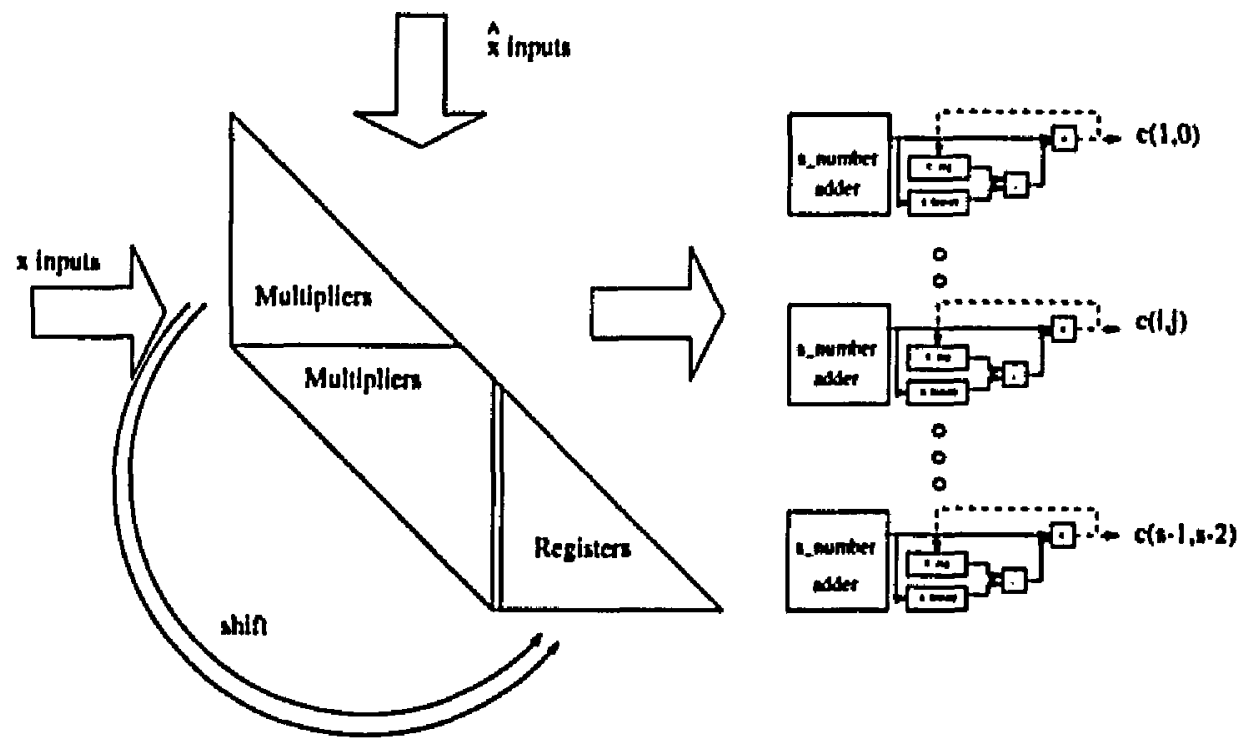

Figure 16: Block configuration for C2 method

$$
T_{C 2}=T_{m u l t}+\left(1+\left[\log _{2} s\right]\right) T_{\text {odd }}
$$

when $s(s-1)$ multipliers are used. In order to add $s$ numbers two parallel configurations can be used. The first configuration is to use a tree of adders, as shown in Fig 17(a). In this case $s+1$ adders are needed for each $c(i, j)$, for a total of $\frac{\Delta(-1)(a+1)}{2}$ adders in the $C$ block. In the second configuration, as shown in Fig 17(b), multiplexors are used to correctly route . the inputs of the $\frac{1}{2}$ adders to sum the $s$ inputs. In this method, for each $c$ coefficient $\frac{1}{2}+2$ adders are required. Total number of adders needed in the $C$ block is $\frac{s(a-1)}{2}\left(\frac{t}{2}+2\right)$. Note 
that the second configuration has the same time delay as the first configuration, with almost half number of the adders.

Another way to calculate the $c$ coefficients is to calculate the bottom row, $c(i, 0)$ for $i=1,2, . . s-1$, or the left column, $c(s-1, j)$ for $j=0,1, . . s-2$, of the $c$ 's triangle, using the values of the corresponding c's from the previous input block. Then the other c's may be obtained by adjusting the value of the c's of that row or column. That is, for the c's on the bottom row

$$
\begin{aligned}
c_{\text {new }}(i, 0)= & c_{\text {old }}(i, 0)+\sum_{k=0}^{-1} x^{*}(n+i-k) \hat{x}(n-k)- \\
& \sum_{k=0}^{k-1} x^{*}(n+i-k-M) \hat{x}(n-k-M) ; \text { for } i=1, \ldots, s-1,
\end{aligned}
$$

and

$$
\begin{aligned}
c_{\text {new }}(k+1, l+1)= & c_{\text {new }}(k, l)+\hat{x}(k+1) x(l+1)-\hat{x}(k-M) x(l-M) \\
& \text { for } k=1, \ldots, s-1, \text { and } l=0,1, \ldots, k-1 .
\end{aligned}
$$

Or for the c's on the left column

$$
\begin{aligned}
c_{\text {new }}(s-1, j)= & c_{\text {old }}(s-1, j)+\sum_{k=0}^{-1} x^{*}(n+s-k) \hat{x}(n+j-k)- \\
& \sum_{k=0}^{\infty-1} x^{*}(n+s-k-M) \hat{x}(n+j-k-M) \\
& \text { for } j=0,1, \ldots, s-2,
\end{aligned}
$$

and

$$
\begin{aligned}
c_{\text {new }}(k-1, l-1)= & c_{\text {new }}(k, l)-x^{*}(k) \hat{x}(l)+x^{*}(k-M) \hat{x}(l-M) \\
& \text { for } k=1, \ldots, s-1, \text { and } l=0,1, \ldots, k-1 .
\end{aligned}
$$




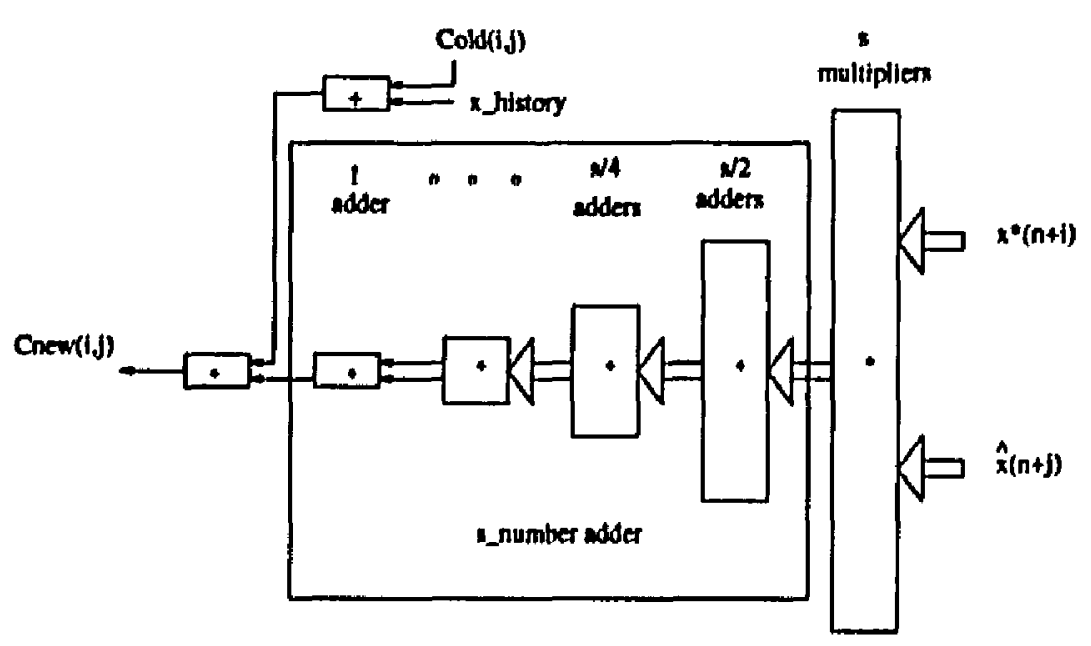

Fig. 17(a)

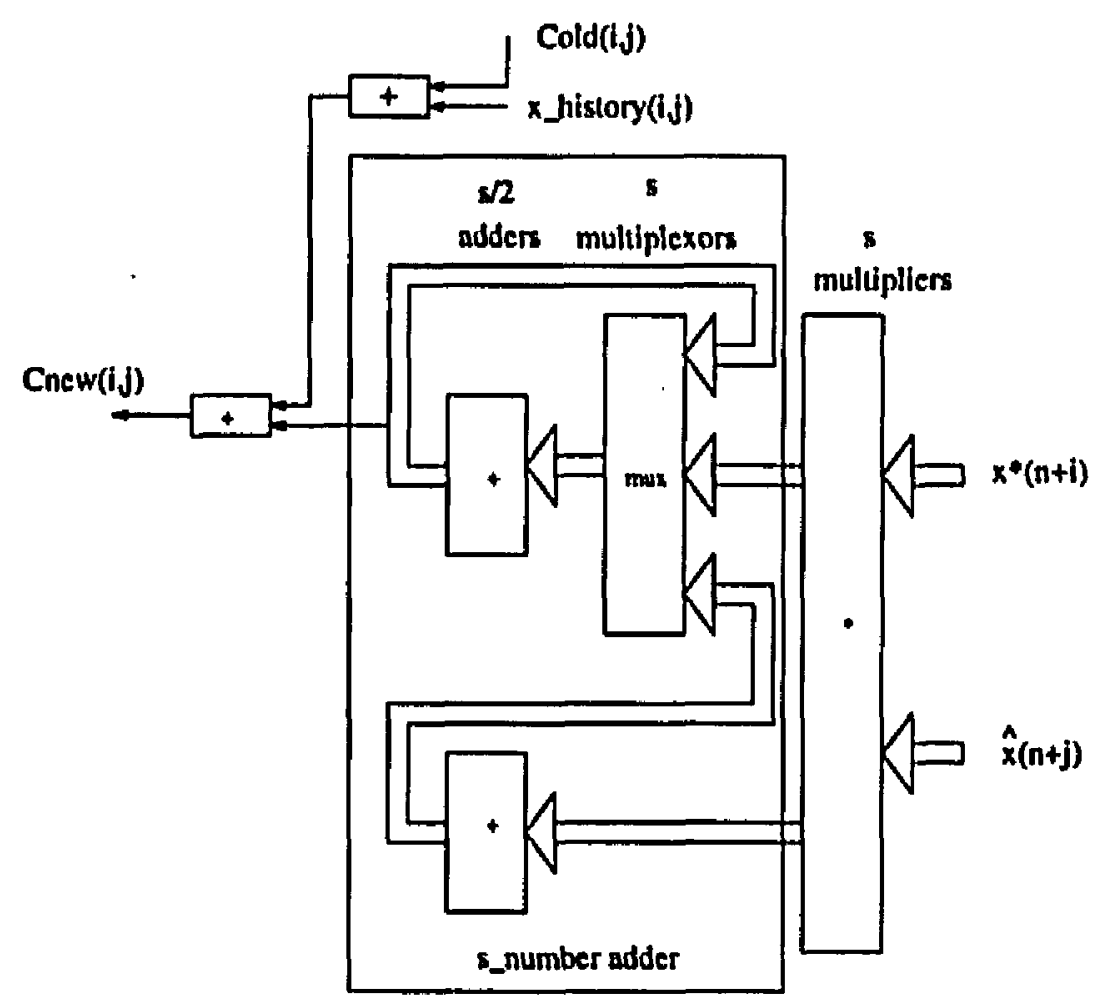

Fig. 17(b)

Figure 17: Two configurations for an $s . n u m b e r$ adder (a) $s . n u m b e r$ adder without multiplexors (b) s_number adder with multiplexors 
An architecture for these equations, call it $\mathrm{C} 3$, would require $s(s-1)$ multiplications and $\frac{3 s(x-1)}{2}$ additions. Time to calculate all $c$ 's using $s(s-1)$ multipliers and $s-l$ adders is

$$
T_{C 3}=T_{\text {mult }}+4(s-1) T_{a d d} .
$$

Figure 18 depicts the block diagram of calculating one of the $c(i, j)$ in $\mathrm{C}$ block using the C3 archilecture.

In this method, the $c$ values on each diagonal are calculated using the values of the prior c's located on the same diagonal on the bottom row of the lower triangle. To calculates the new values of the c's on the bottom row of the upper triangie, Eq. 5.6, first, add the products of the multipliers on each diagonal to the $C_{\text {old }}$. Then, subtract the values of $\frac{M}{8}$ multiplication prior. The other $c$ 's on cach diagonal are obtained from the values of the $c$ 's on the bottom row of the upper triangle, Eq. 5.7.

Another recursive way to calculate the $c$ coefficients, call it C4, is to use the $c$ 's on the left column of the previous block and compute the $c$ 's on the bottom row , i.e.,

$$
\begin{aligned}
c_{\text {new }}(i, 0)= & c_{\text {old }}(s-1, s-i-1)+\sum_{k=0}^{i} x^{*}(n+i-k) \hat{x}(n-k)- \\
& \sum_{k=0}^{i} x^{*}(n+i-k-M) \hat{x}(n-k-M) ; \text { for } i=1, \ldots, s-1,
\end{aligned}
$$

The other $c$ 's in each diagonal can then be calculated as in equation 5.5. Figure 19 shows the configuration of the $\mathrm{C}$ block, using $\mathrm{C} 4$ method, In this method, the values of $\mathrm{c}$ 's on each diagonal are calculated using the values of the prior $c$ 's on the same diagonal on the left column of the lower triangle. Adding the results of the multipliers on each diagonal and subtracting the values of prior multiplications calculates the new values of the $c$ 's, Eq. 5.8. In this method $s(s-1)$ multiplications and $(s+1)(s-1)$ additions are performed. Using $s(s-1)$ multipliers and $s-1$ adders, the delay time of this method is 


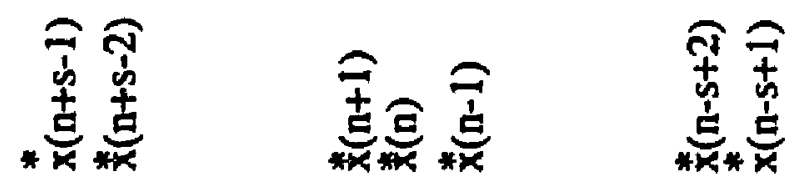

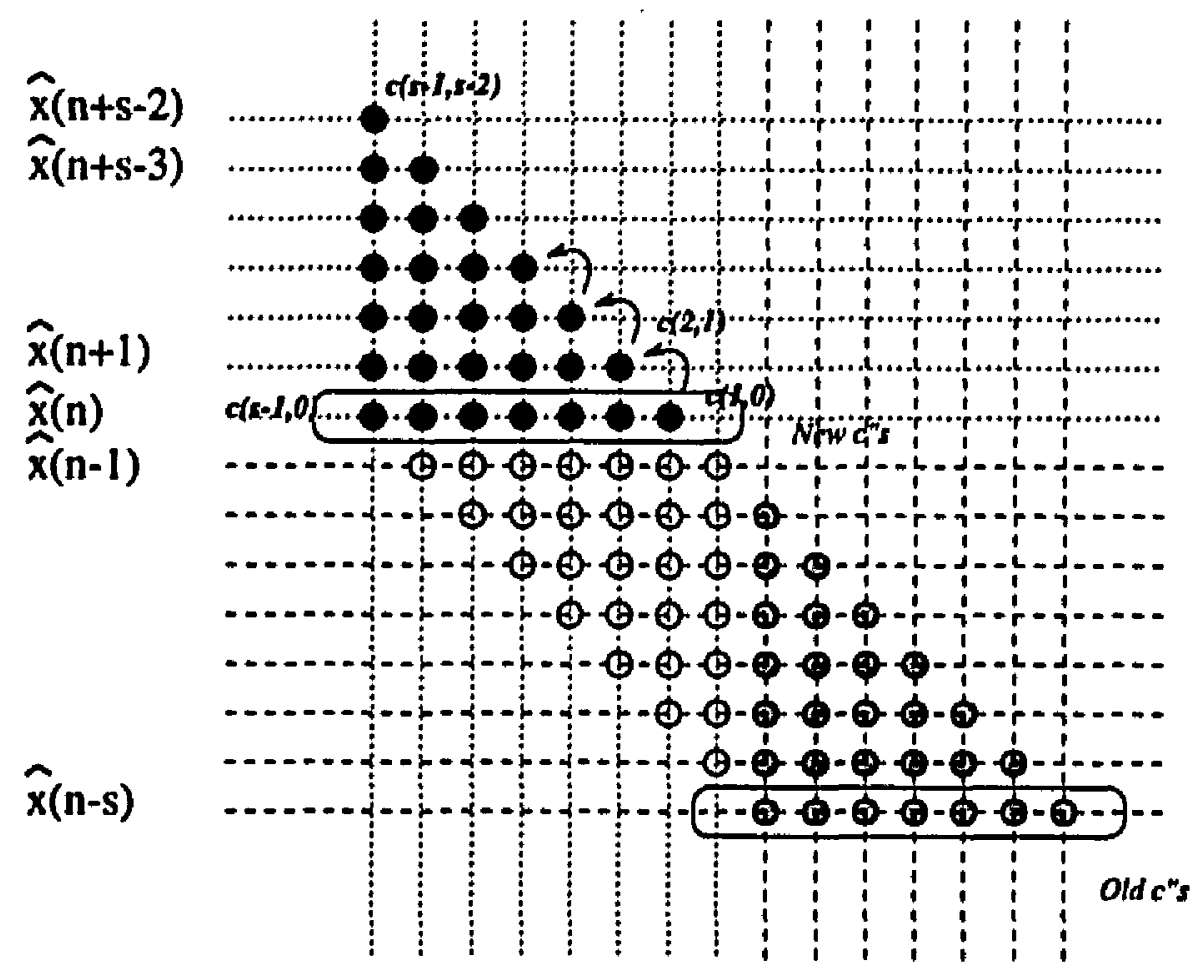

Figure 18: Block configuration of $\mathrm{C} 3$ method

$T_{C 4}=T_{\text {mult }}+2 s T_{\text {add }}$

In the fifth architecture for the $\mathrm{C}$ block, $\mathrm{C}$, all new $c$ coefficients are calculated, using the value of the c's on the left column of the previous block, Fig. 20.

$$
\begin{aligned}
c_{n e w}(i, j)= & c_{\text {old }}(s-1, s-i-1)+\sum_{k=0}^{i} x^{*}(n+i-k) \hat{x}(n+j-k)- \\
& \sum_{k=0}^{i} x^{*}(n+i-k-M) \hat{x}(n+j k-M) ; \text { for } i=1, \ldots, s-1,
\end{aligned}
$$

Each diagonal of Fig. 20 has a structure shown in Fig. 21. The propare values of 

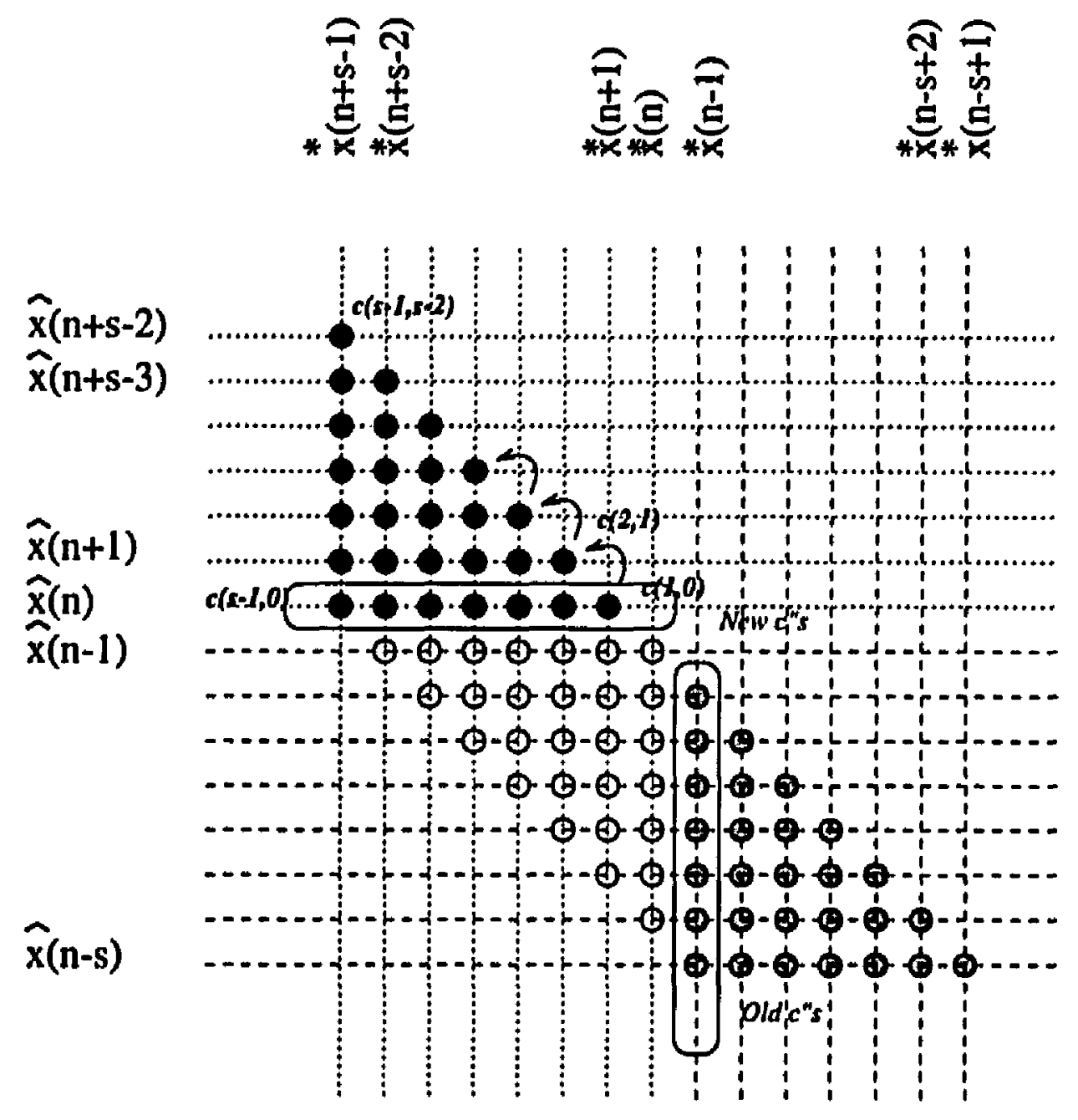

Figure 19: Block configuration of $\mathrm{C} 4$ method

$x^{*}(i)$ and $\bar{x}(j)$ are set up on the inputs of the multipliers. The result of the multiplication is shifted into the shift register. The values on top of the shift register is accumulated in the $+\Sigma$ adder. The values on the bottom of shift register is accumulated in the $-\Sigma$ adder. These adders keep the values of the summations in Eq. 5.9. On each diagonal, the values of the $c$ 's on the left column of the previous block are used to calculate the values of the other c's on that diagonal. Thus, the values of the old $c$ 's on the left column is latched from the top of CSR shift register. The new value of the c's, outputs of the subtractors, are saved into 

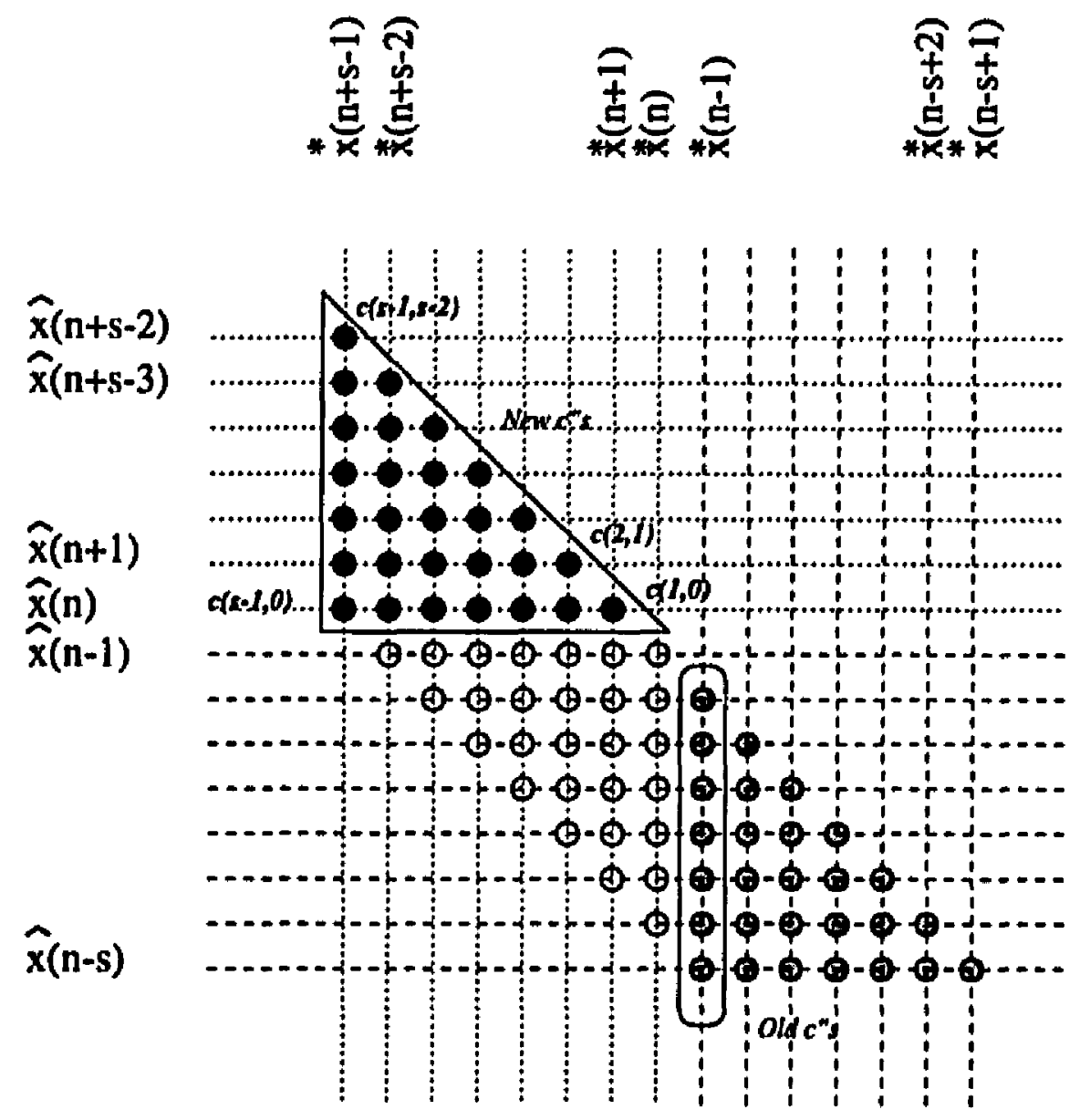

Figure 20: Block configuration of C5 method

the CSR shift registers.

This architecture of the $C$ block needs $s(s-1)$ multiplications and $\frac{(s-1)\left(2 s^{2}-7 c-6\right)}{6}$ additions. This configuration of the $C$ block requires $s-1$ multipliers and $4(s-1)$ adders. The minimum time delay of the $\mathrm{C}$ block, using this architecture, is

$T_{C S}=s^{\prime} T_{m u l t}+3 T_{\text {add }}$

Table 5 depicts a comparison among five architectures for the $\mathrm{C}$ block.

Figure 22 show a comparison among these five methods of implementing the $\mathrm{C}$ block. In this figure, it is assumed that for floating point numbers a multiplication time is equal an 
Table 5: Comparison of the complexity and time delay for the $\mathrm{C}$ block calculation

\begin{tabular}{|c|c|c|c|c|c|c|}
\hline $\begin{array}{l}\text { Nethat's } \\
\text { name }\end{array}$ & $\begin{array}{l}\text { Tiquastion } \\
\text { mimber(u) }\end{array}$ & \multicolumn{2}{|c|}{ 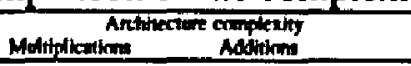 } & \multicolumn{3}{|c|}{ 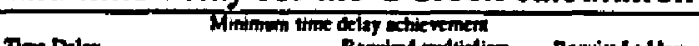 } \\
\hline CI & 4.12 & $\frac{(1-1)(2 M+1-2)}{2}$ & $\frac{(u-1)(M-1)}{2}$ & $T_{m u l l}+(\ln z, M) T_{\text {add }}$ & $\frac{(t-1)(2 M t+-2)}{2}$ & $\frac{(1-1)(M-1)}{2}$ \\
\hline $\mathbf{C 2}$ & 3.1 & $\cdot(\cdot-1)$ &.$^{2}(x-1)$ & $T_{m \text { malt }}+\left(1+\operatorname{Rnn_{2}} d\right) T_{\text {odd }}$ & $2(0-1)$ & $\frac{(e-1)(e+4)}{4}$ \\
\hline $\mathbf{C o}$ & s.4ss & . & & & & \\
\hline & $n 9 k 3.7$ & $(\cdot(\cdot-1)$ & $\frac{3(k-1)}{2}$ & $T_{\text {mull }}+4(-1) T_{\text {add }}$ & $\cdot(\cdot-1)$ &.-1 \\
\hline ca & s.w3s & $(a-1)$ & $(0+1)(0-1)$ & $T_{\text {mult }}+2 o T_{a d d}$ & $\cdot(\cdot-1)$ &.-1 \\
\hline $\mathrm{cs}$ & $\$ .9$ & $\cdot(0-1)$ & $\frac{(-1)\left(2^{2}-21-6\right)}{6}$ & $-T_{m u l t}+3 T_{a d d}$ &.-1 & $4(1-1)$ \\
\hline
\end{tabular}

addition time. This time is called $T_{\text {unit }}$,

$$
T_{u n i t}=T_{\text {mult }}=T_{\text {add }}
$$

It is also assumed that the area of a multiplier for two floating point numbers is approximatcly the same as the area of an adder for two floating point numbers. This area is called $A_{\text {unit. }}$.

$$
A_{\text {unit }}=A_{\text {mult }}=A_{\text {add }}
$$

Time_area unit is defined as the product of a unit time and the unit area,

$$
T A_{\text {unit }}=T_{\text {unit }} * A_{\text {unit }} \text {. }
$$

The curves shown in Fig. 22 are for a filter with a constant order of $128, M=128$. Fig. 22(a) shows the time delay of the $\mathrm{C}$ block's methods versus different step sizes $s$. Fig. 22(b) shows the total number of units, number of multipliers plus number of adders, required to build the $\mathrm{C}$ block versus step sizes $s$. Fig. 22(c) depicts the time_area performance of different $C$ block's methods with respect to step sizes $s$.

With the same assumptions, Eq. 5.10, 5.11, and 5.12, the effect of the order of the filter, $M$, for different methods of block $\mathrm{C}$ is shown in Fig. 23. In this figure the step size 
is constant and it is equal to $8, s=8$.

As figures 22 and 23 show $\mathrm{Cl}$ and $\mathrm{C} 2$ are the fastest methods to calculate coefficients. However $\mathrm{C5}$ is the smallest configuration for the $\mathrm{C}$ block. Time.Area criteria shows that $\mathrm{C} 2$ and $\mathrm{C5}$ are the best in terms of time area among these five methods. The over all performance of PBS LMS using C2 and C5 will be investigaled in the section 5.5 . 


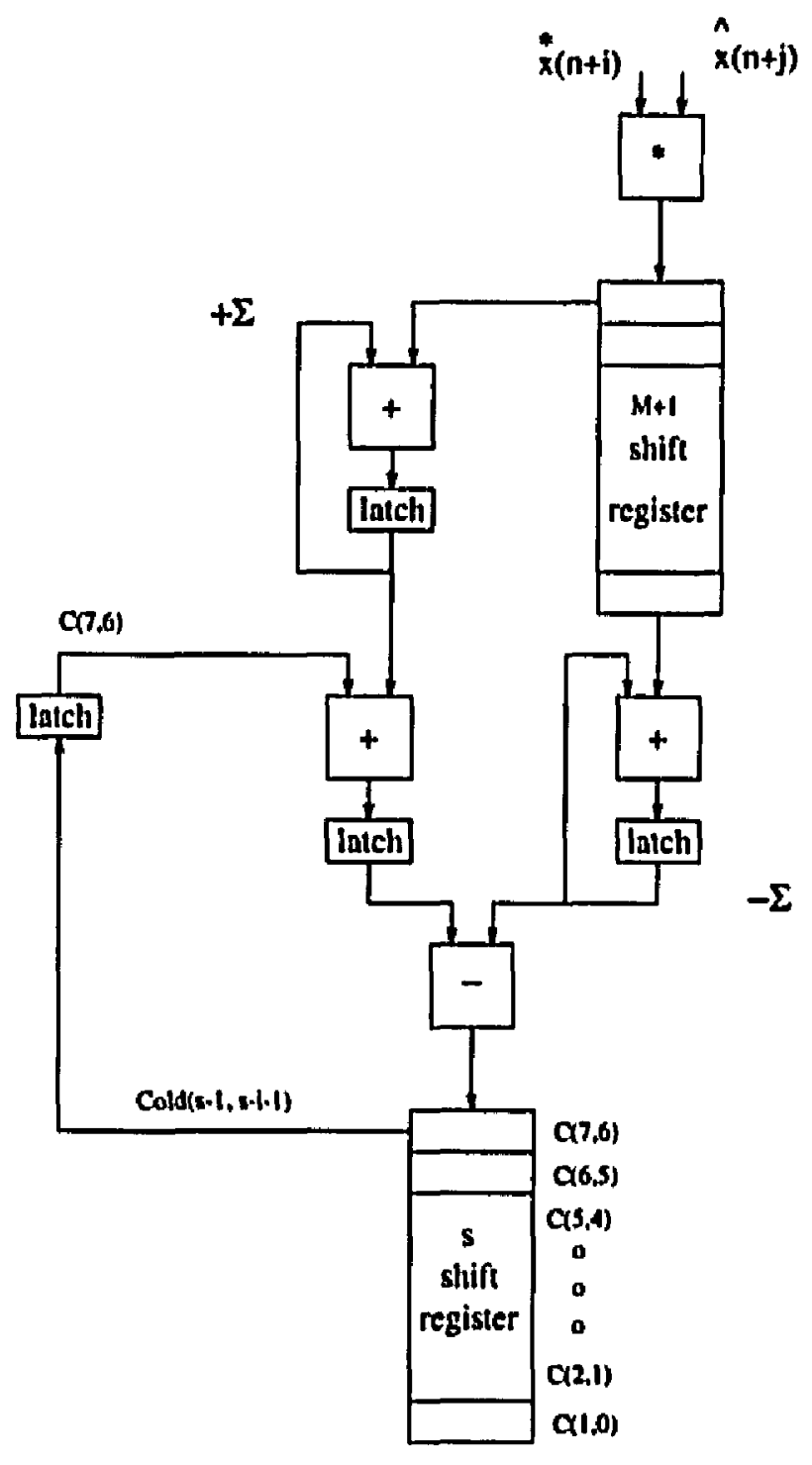

Figure 21: Configuration of one of the diagonal of the $\mathrm{C}$ block 


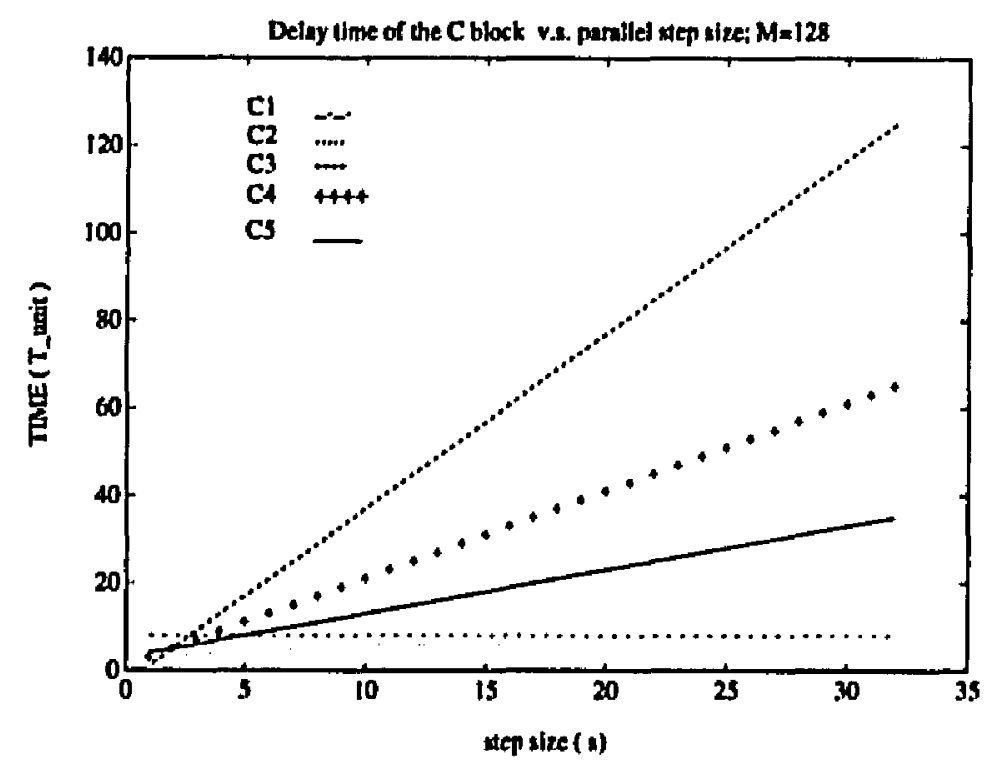

(a)

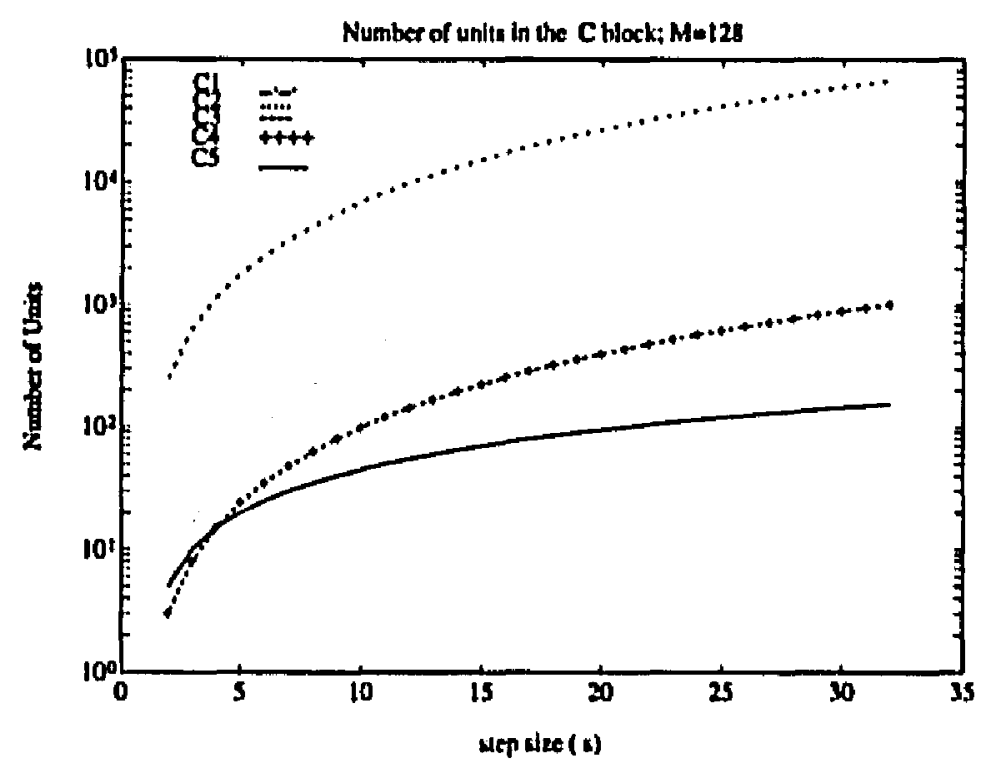

(b)

Figure 22: Comparison among the different C block's methods. (a) Total time delay for the $\mathrm{C}$ block (b) Total area required for the $\mathrm{C}$ block (c) Time_area presentation of different $\mathrm{C}$ 's 
Fig. 22 (continued)

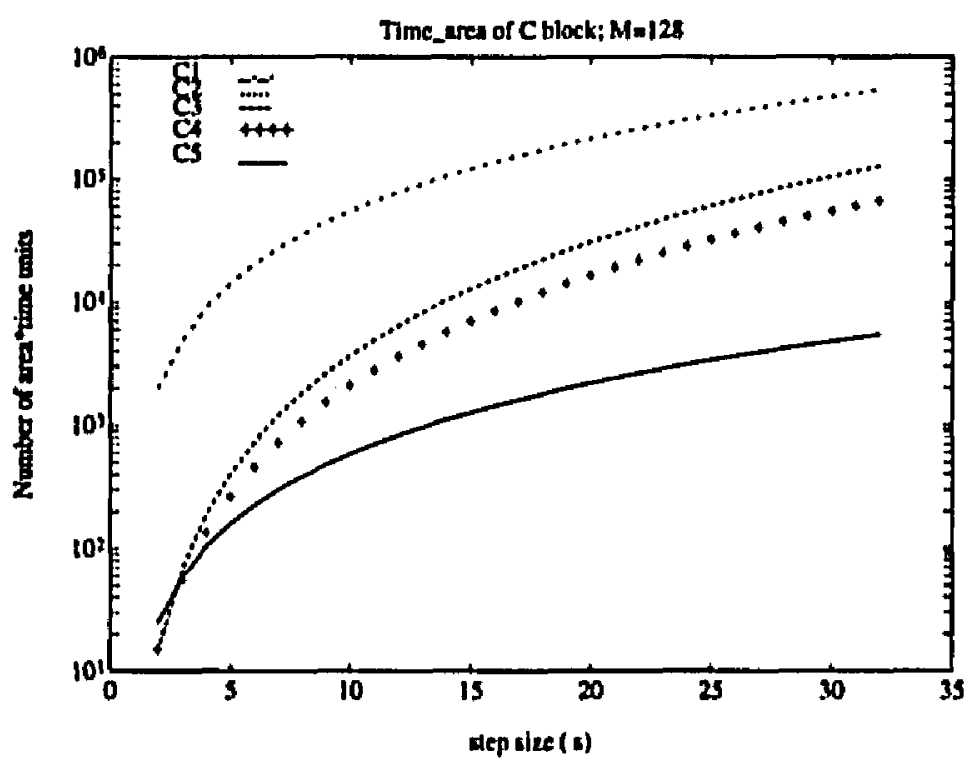

(c) 


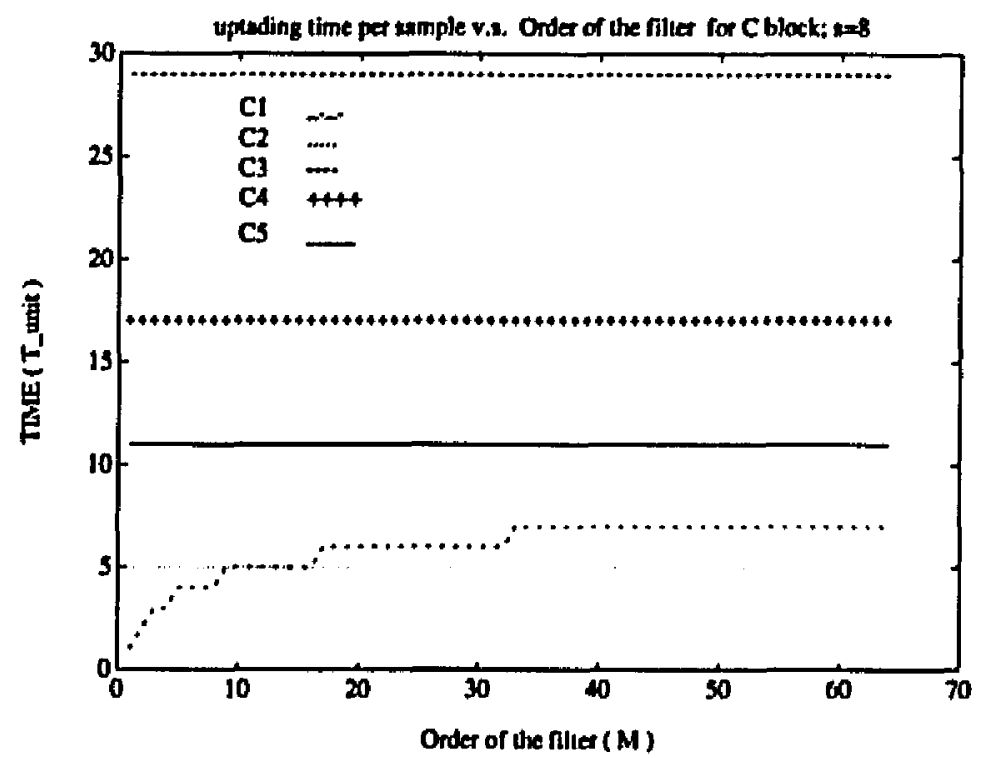

(a)

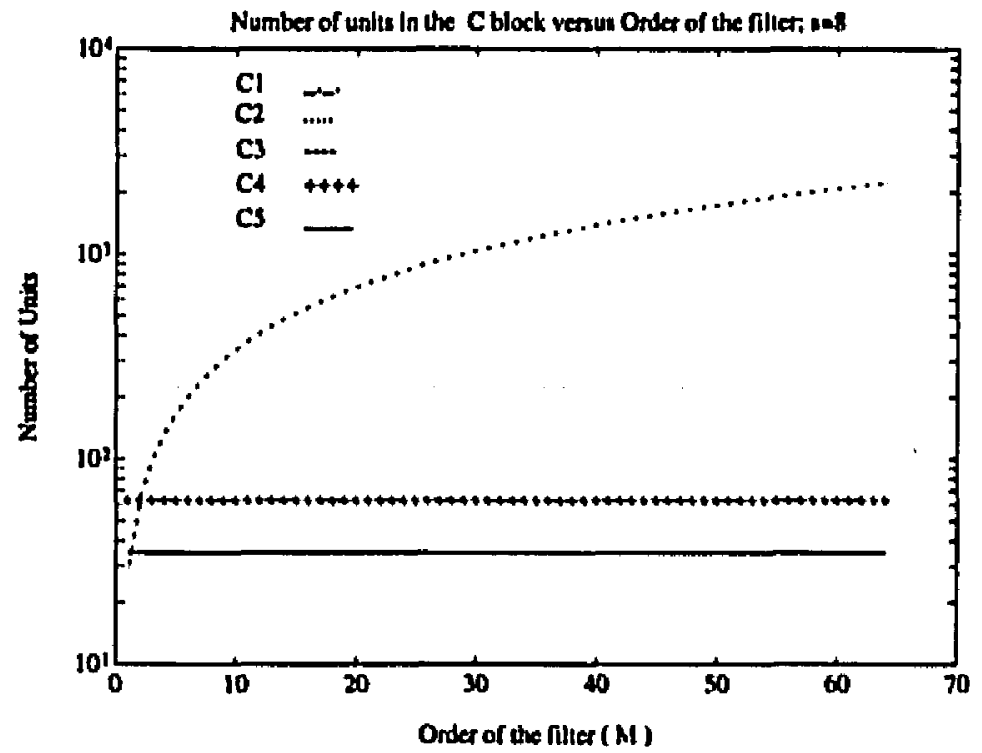

(b)

Figure 23: Comparison among the different $\mathrm{C}$ block's methods versus order of the filter. (a) Total time delay for the $\mathrm{C}$ block. (b) Total area required for the $\mathrm{C}$ block. (c) Time_area presentation of different C's. 
Fig. 23 (continued)

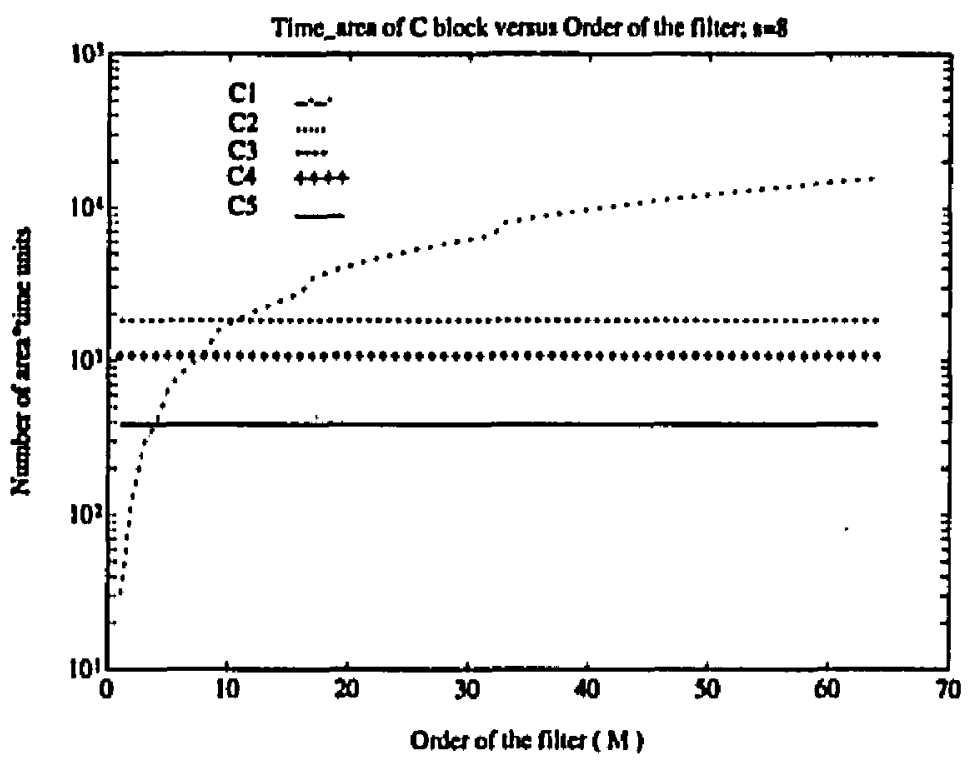

(c) 


\subsection{Evaluation of the $P$ block:}

The scalar values of $p$ have been defined in 4.7, and are calculated in block P of figure 6 . For $s$ step update, there are $s$ coefficients of $p$ as shown in figure 24, each requiring $M$ multiplications and $M$ additions.

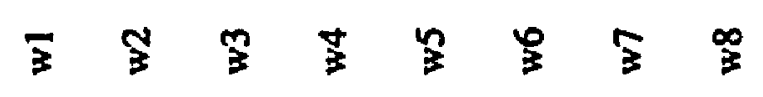

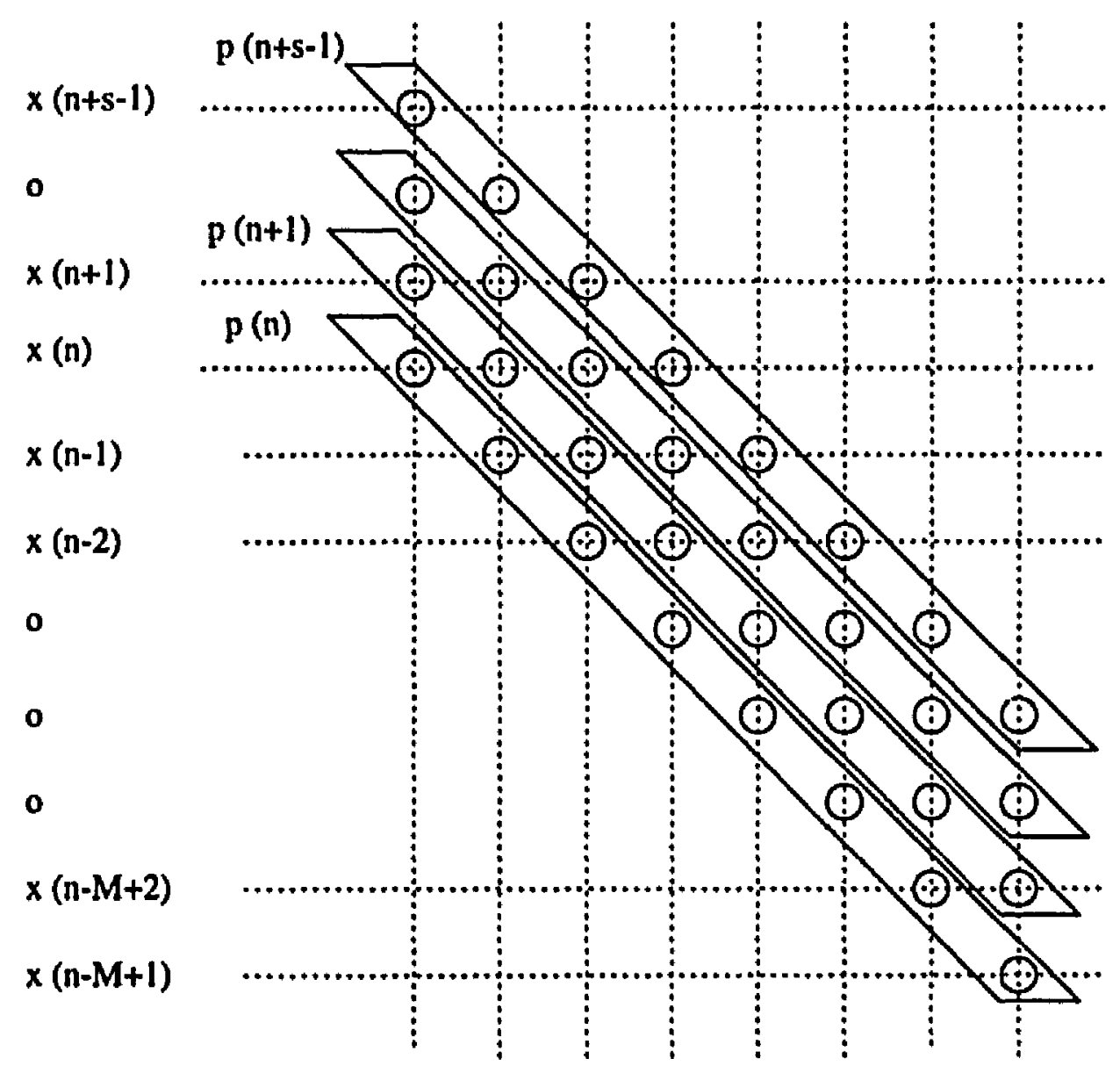

Figure 24: $\mathrm{P}$ block configuration

The first approach to calculate the $p$ 's is a direct method using $M$ multipliers and 
$M$ adders for each $p$ for a total of . $s M$ multipliers and $s M$ adders. That is, for each $p(s i), s i=0,1, \ldots, s-1$, use $M$ multipliers to obtain $M$ values of $p x(s i, k), k=1,2, . ., M$, simultancously,

$$
p x(s i, k)=w_{k}(n) x(n+s i+1-k) \text { for } k=1,2, . ., M .
$$

Then use a tree of adders to calculate the summation of $p x(s i, k)$ 's to obtain $p y(s i)$,

$$
p y(s i)=\sum_{k=1}^{M} p x(s i, k)
$$

Each of these trees of adders contains $M-1$ adders. A single adder at the end is needed to subtract $p y(s i)$ from $d(s i)$ to produce $p(s i)$,

$$
p(s i)=p y(s i)-d(s i)
$$

Equation 5.13 requires $M$ multipliers and has a delay time equal to $T_{\text {mult }}$. Equation 5.14 needs $M-1$ adders with $\left\lceil\log _{2} M\right\rceil T_{\text {add }}$ delay time. Equation 5.15 needs one adder with one $T_{\text {tudd }}$ delay time. Therefore, each $p($ si $)$ needs $M$ multipliers plus $M$ adders. Since in this method all $p(s i)$ 's are calculated concurrently, the $P$ block requires $s M$ multipliers and $s M$ adders.

The total time delay of block $P$ is equal to the time of one multiplication plus the time of adding $M$ numbers plus one subtraction,

$$
T_{P I}=T_{\text {mull }}+\left(1+\left[\log _{2} M\right\rceil\right) T_{\text {add }}
$$

Figure 25 shows the direct implementation of the P block for one of $s$ values of $p(s i)$ 's.

In the second approach the number of adders is reduced by half, $\frac{\mu l}{2}$ adders for each $p(s i)$, by multiplexing the inputs to the adders. This approach requires total of $s\left(\frac{M}{2}+1\right)$ 


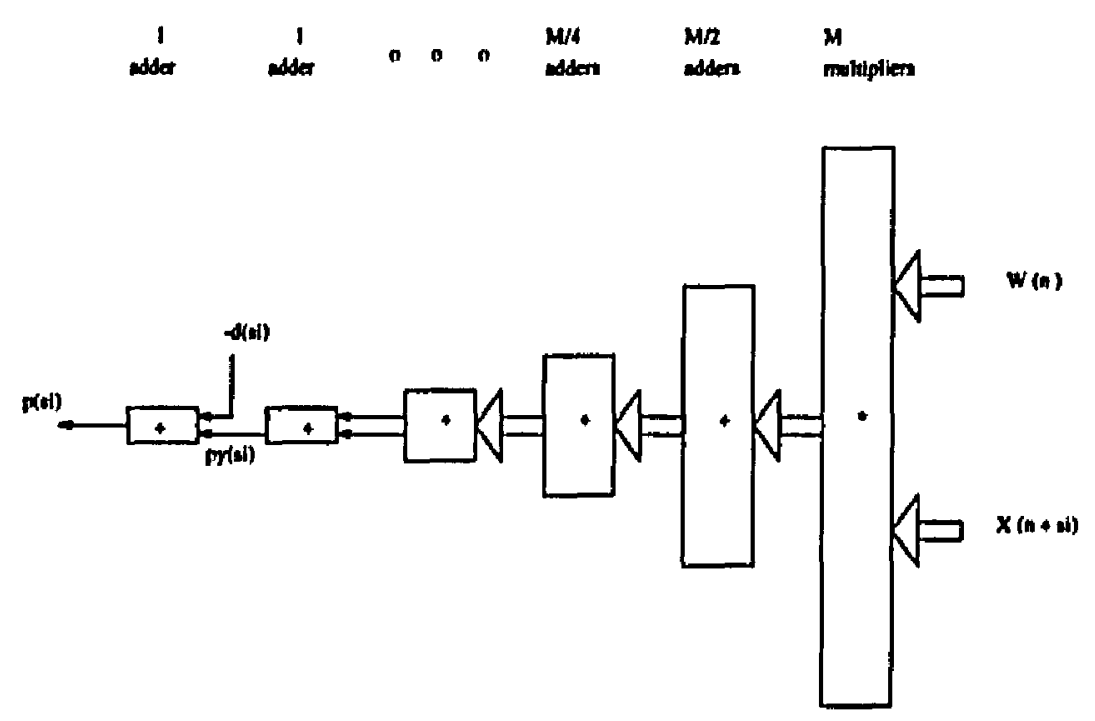

Figure 25: P1 block configuration for one of $p(s i)$ 's.

adders and $s M$ multipliers. Figure 26 depicts the schematic diagram for one of $p(s i)$ 's. The delay time of this method is the same as the first approach,

$$
T_{P 2}=T_{\text {mult }}+\left(1+\left\lceil\log _{2} M\right\rceil\right) T_{\text {add }} .
$$

The third and fourth methods are two recursive solutions for the $\mathrm{P}$ block. $p$ coefficients can be calculated recursively by using only one multiplication and addition structure, instead of the $s$ copies, of Fig. 25 or 26. In this way the number of hardware units is reduced.

The configuration of $\mathrm{P3}$, a pipelined method to implement the $\mathrm{P}$ block, is shown in Fig. 27. In this case the $\mathrm{P}$ block consists of $M$ multipliers and $M$ adders with latches between each stage. Using this method, the time delay of the $P$ block to calculate the last $p, p(s-1)$, is

$$
T_{P 3}=s T_{\text {mult }}+\left(1+\left\lceil\log _{2} M\right\rceil\right) T_{\text {add }}
$$

Pjpelining the $\mathrm{P}$ architecture of Fig. 26 renders a new structure for the $\mathrm{P}$ block, P4, 


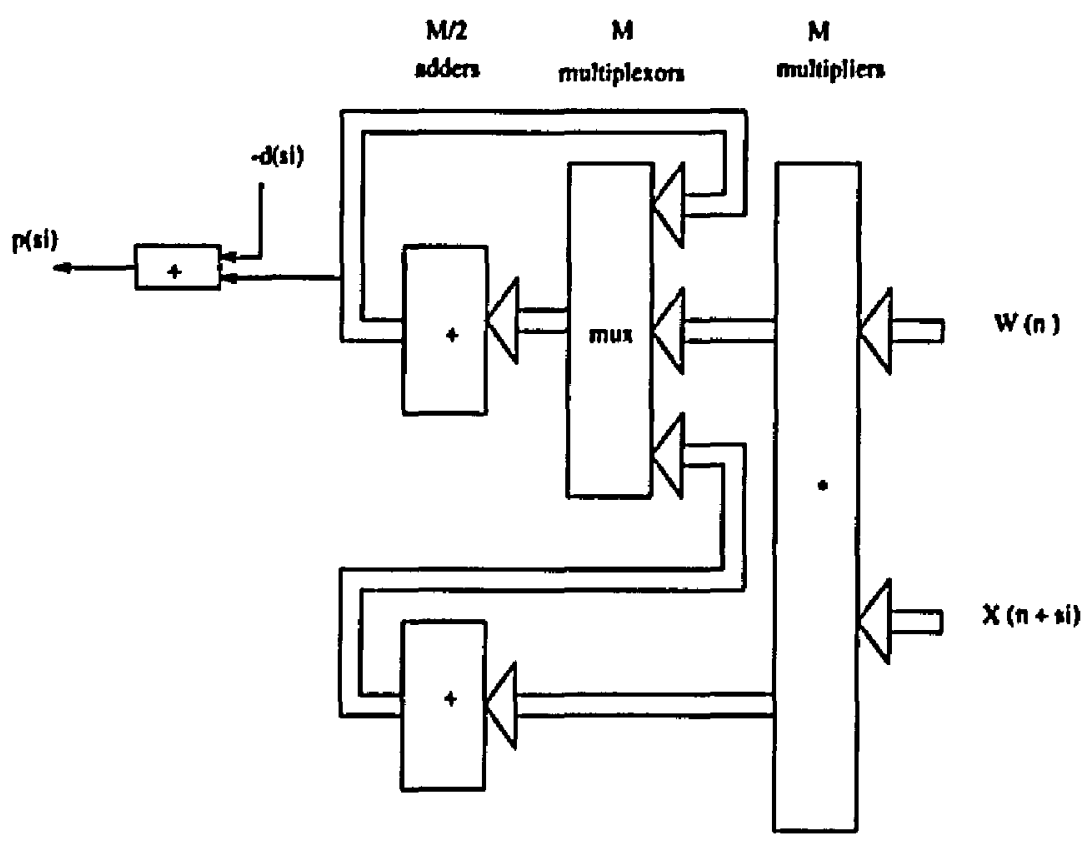

Figure 26: P2 block configuration for one of $p(s i)$ 's.

which is shown in Fig. 28. In this architecture, inputs are latched, multiplexed, and then recursively summed. While summing the first inputs, the next inputs are latclied and multiplexed. When the first sum is computed, it is latched into the final adder, At the same time, the summation of the result of the second multiplication starts. Then the third inputs are latched into the multipliers. This process continues for the $s$ inputs to be latched in, multiplied, summed, and latched out. This structure needs total of $M$ multipliers, plus $\left(\frac{M}{2}+1\right)$ adders and $\frac{M}{2}$ multiplexors. It has a time delay of

$$
T_{P 4}=T_{\text {mult }}+\left(1+s\left[\log _{2} M\right]\right) T_{\text {add }}
$$

Table 6 shows a summary of four methods to calculate p's. Considering the same assumptions made in presentation of the $C$ blocks, 5.10, 5.11, and 5.12, Fig. 29 shows the delay time, required area, and time-area performance of the different methods of 


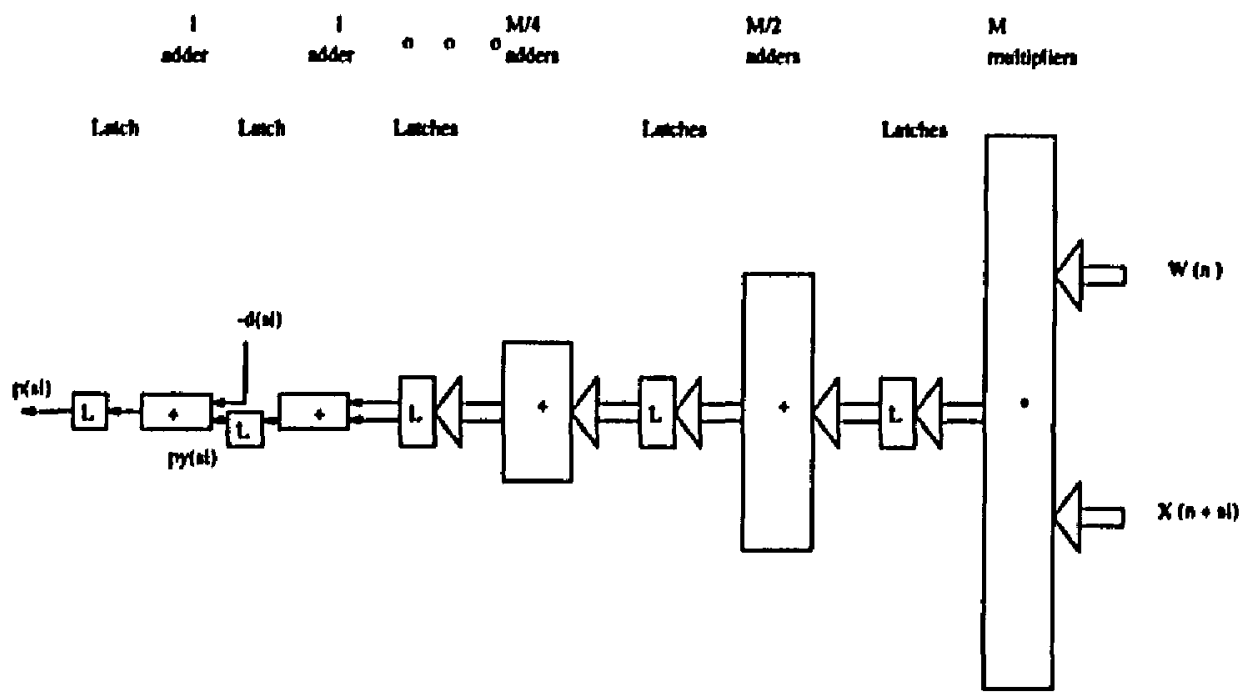

Figure 27: Block configuration of the P3 method

implementing the $\mathrm{P}$ block versus the step size $s$. In this figure the order of the filter is kept constant and equal to 128.

Table 6: Comparison of the four method to implement the $P$ block

\begin{tabular}{|c|c|c|c|c|c|c|c|}
\hline $\begin{array}{l}\text { Therinds } \\
\text { mene }\end{array}$ & Thetrint: & 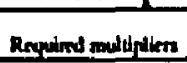 & 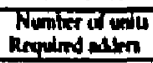 & Rnguind muldirinearm & Mulupticalikin & 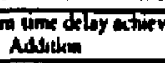 & mulifineting \\
\hline $\mathbf{m}$ & Mi. 25 & $A M$ & - $A$ & o & 1 & $1+\left|N_{w_{2}} M\right|$ & o \\
\hline$m$ & Mres & on & $\left(\left[\frac{4}{x}\right]+1\right)$ & -「팔| & t & $1+\left|n_{\psi_{2}} \mu\right|$ & $\left.\ln _{10} x_{1}\right)$ \\
\hline p) & $\begin{array}{l}\text { Piprelined of } \\
\text { Ple. } 25\end{array}$ & $\boldsymbol{M}$ & $\mu$ & 0 & - & $\left.1+\ln _{2}{ }^{\prime} \mid 1\right)$ & 0 \\
\hline M & $\begin{array}{l}\text { Pijprlined of } \\
\text { Pit. } 26\end{array}$ & $\boldsymbol{M}$ & $\left.r^{4}{ }^{4}\right]+1$ & 141 & 1 & $\left.1+\cdot \mathrm{Au}_{1}, \mathrm{~N}\right)$ & $\cdot\left|N_{v_{2}} N\right|$ \\
\hline
\end{tabular}

Fig. 30 depicts the time, area, and time_area performance of four $\mathbf{P}$ methods with respect to order of the filter for constant step size $s=8$.

Comparing the performance of all the architectures of the P block in Fig. 29 and 29 shows that P1 and P2 have the shortest time delay, P1 and P2 have the same delay 


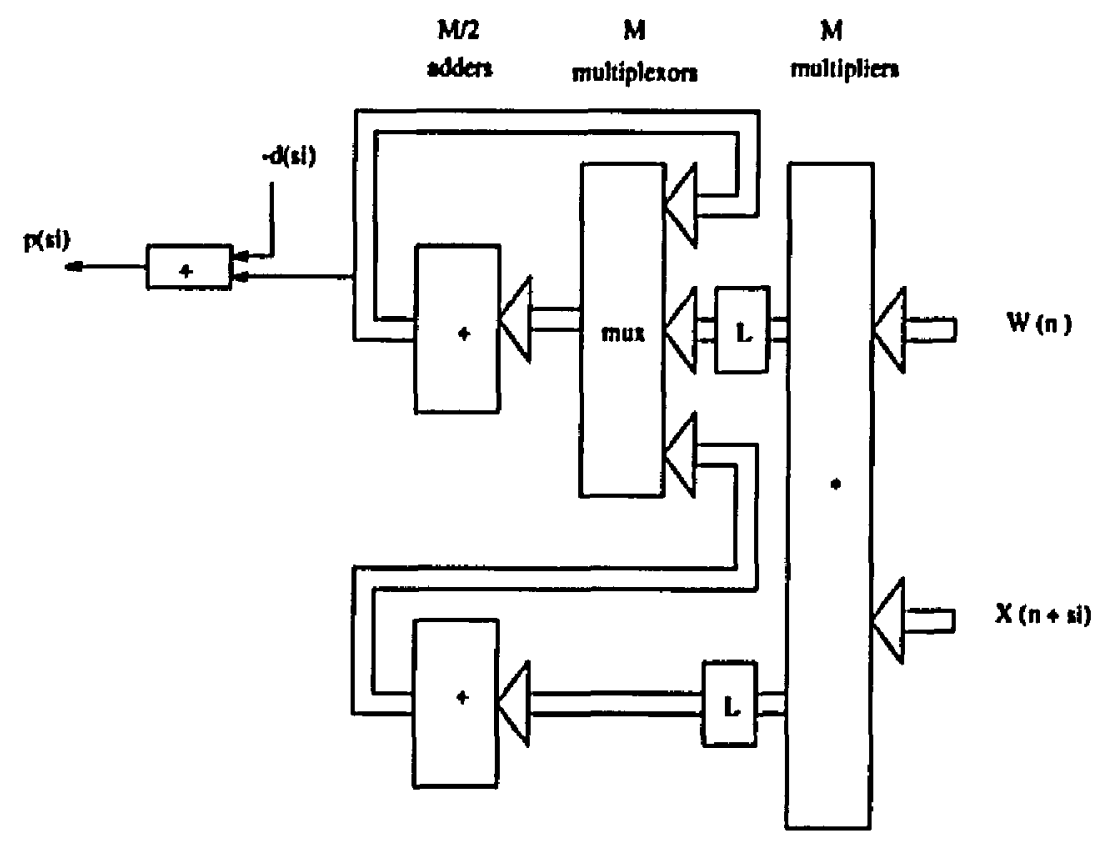

Figure 28: Block configuration of the P3 method

time. The most time consuming methods are P3 and P4. Comparison between two fastest architectures, P1 and P2, shows that P2 needs fewer units than P1. From the delay time point of view $P 2$, is the best architecture for the $P$ block, and is the first choice for the $P$ block.

P3 and P4 architectures are slower as expected. However, P3 and P4 architectures are superior in area and time_area criteria. Fig 29(a) shows that in the delay time comparison, P3 has a better performance than P4. Thus, P3 architecture is selected as the second choice for the P block. Therefore P2, the best fastest architecture, and P3, the best smallest architecture, are two best candidates for the $\mathbf{P}$ block. These two architectures will be used in PBS LMMS implementations. The over all performances of the PBS LMS using either of these methods are investigated in the section 5.5. 


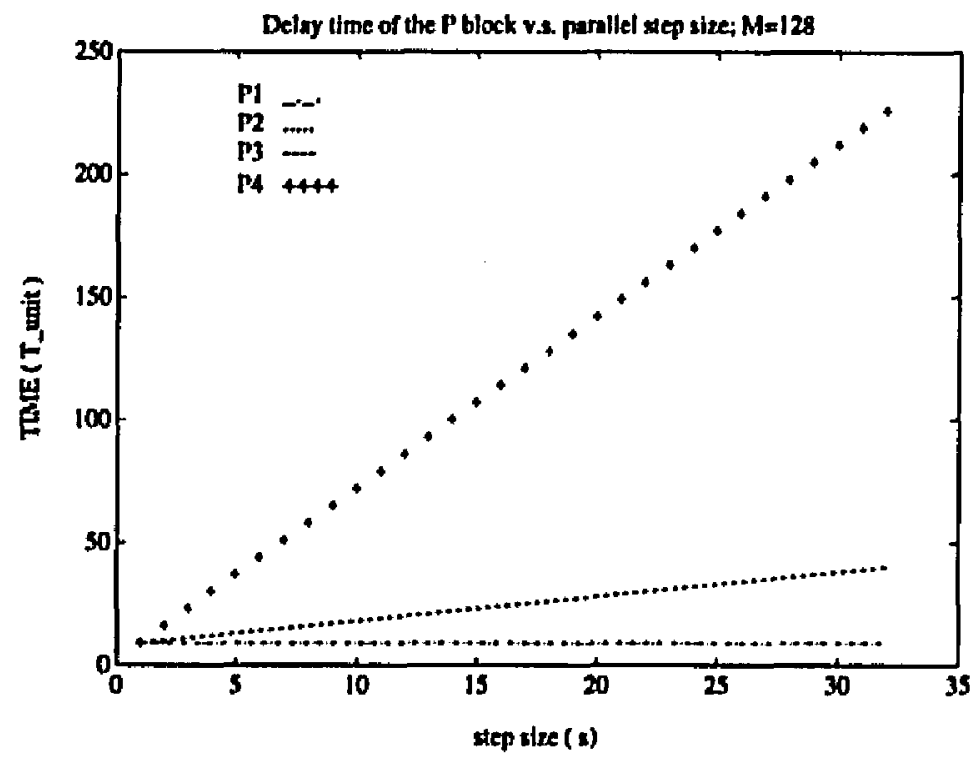

(a)

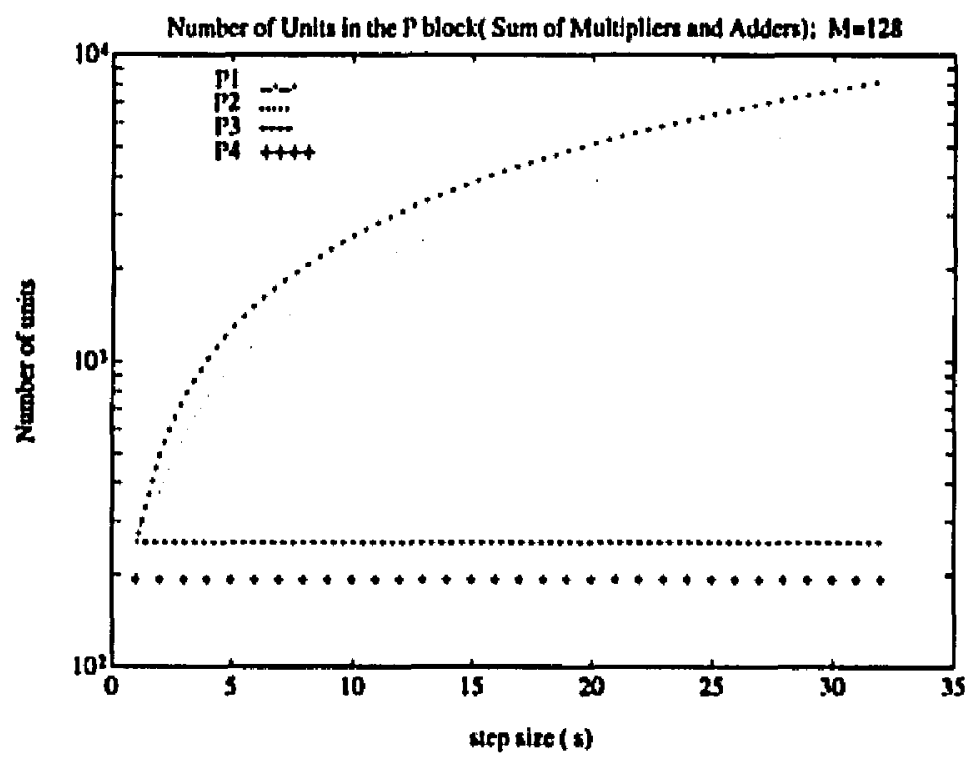

(b)

Figure 29: Comparison among the different P block's methods for constant order of the filter, $M=128$. (a) Total time delay for the $P$ block versus step size $s$. (b) Total area required for the P block versus step size $s$. (c) Time_area presentation of different $P$ 's versus step size $s$ 
Fig. 29 (continued)

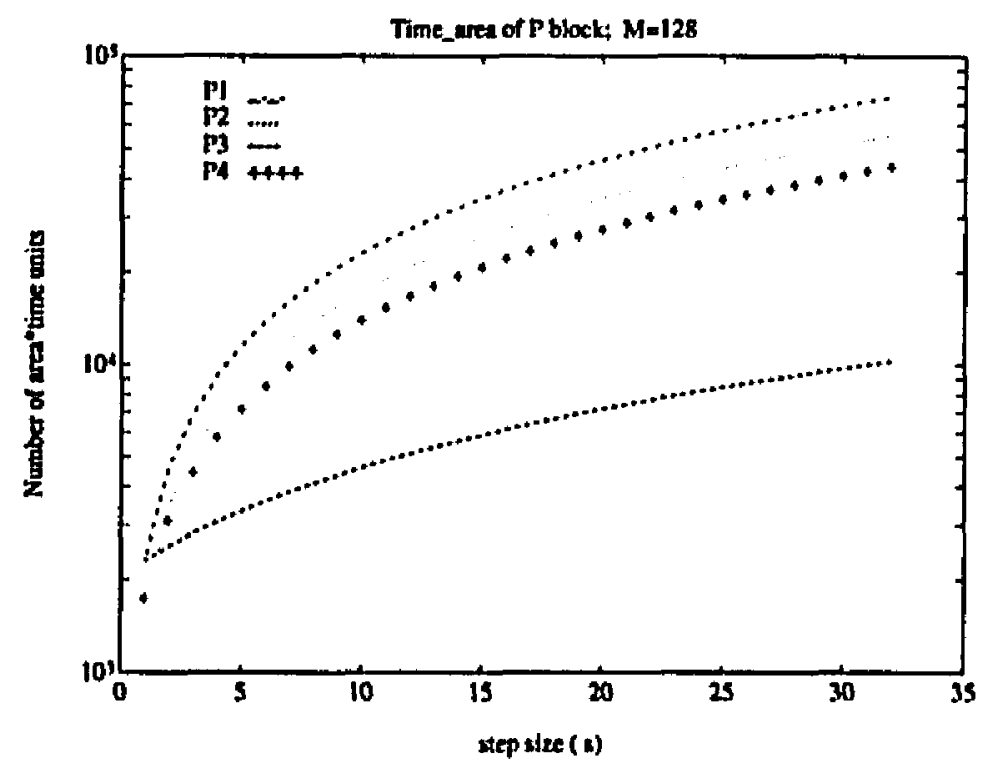

(c)

\subsection{Evaluation of the $\Delta$ block:}

There are different approaches to calculate $\Delta w(n+s)$ in the $\Delta$ block, using the values of $c ' s, p$ 's and x's.

The first approach is to assign one multiplier for each row of $\Delta$ block to calculate $f(r)$, the product of the $c$ and $p$ terms in that row. Refer to the notation defined in the algorithm 2, for all $r$ 's, $0<r<2$

$$
f(r)=\left\{\begin{array}{lc}
p\left(n+a_{r}\right) & \text { if } r=2^{a_{r}} \\
{\left[\prod_{(i, j) \in C_{r}} c(i, j)\right] p\left(n+a_{r}\right)} & \text { otherwise }
\end{array}\right.
$$




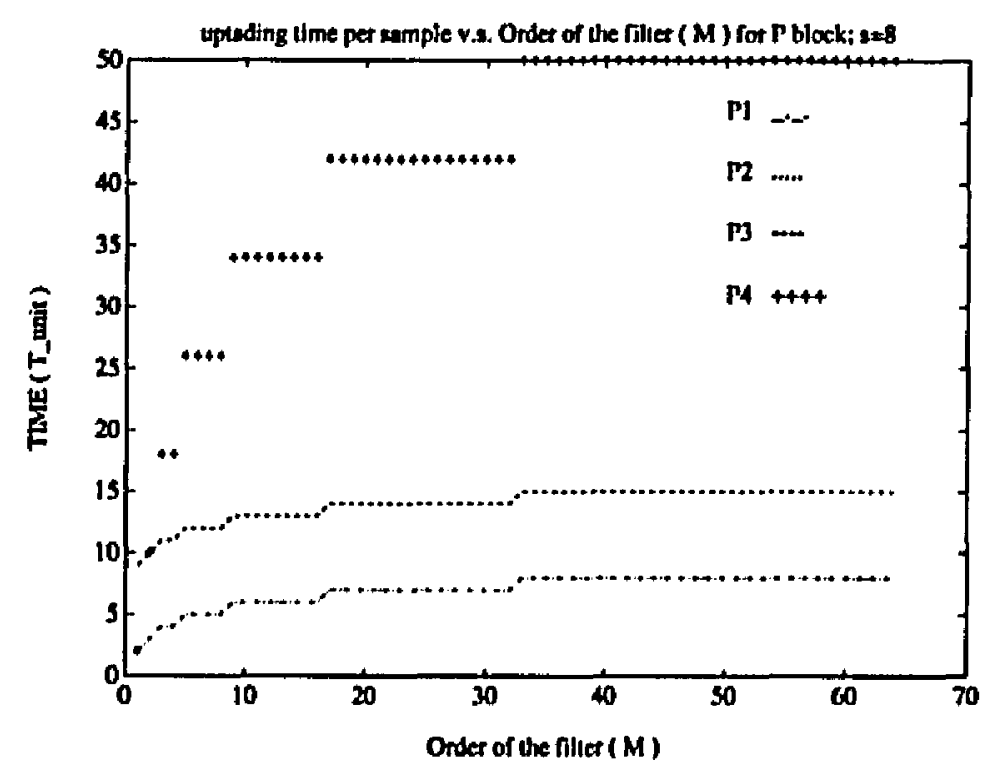

(a)

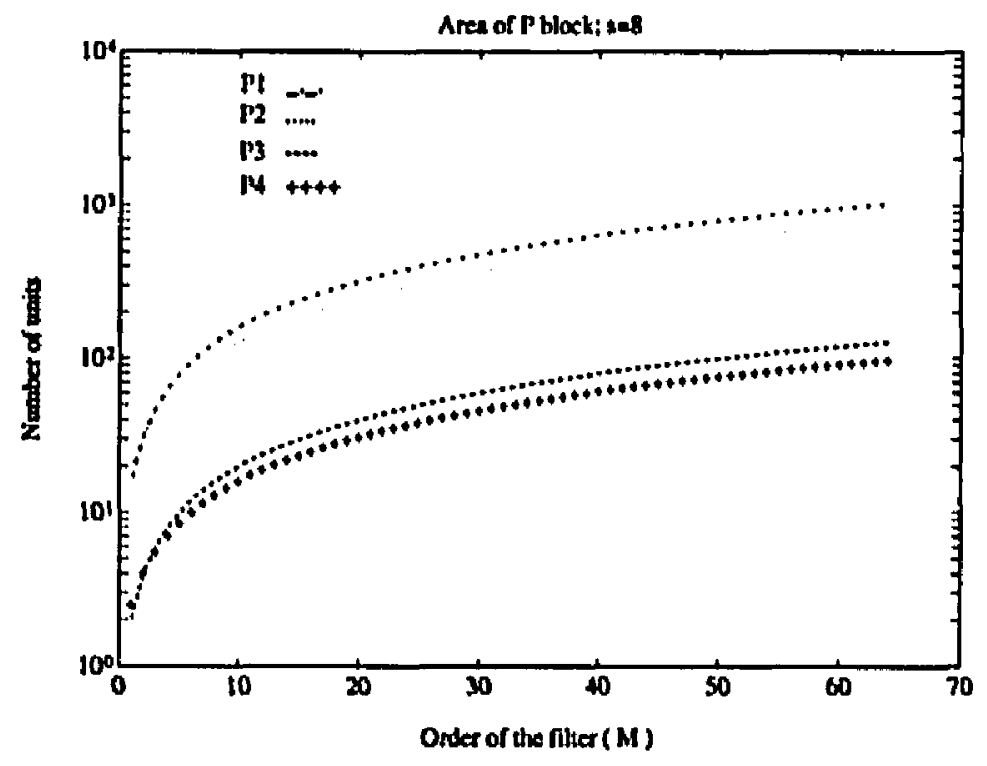

(b)

Figure 30: Comparison among the different $P$ block's methods for constant step size, $s=8$. (a) Total time delay for the P block versus order of the filter (b) Total area required for the P block versus order of the filter (c) Time.area presentation of different P's versus order of the filter 
Fig. 30 (continued)

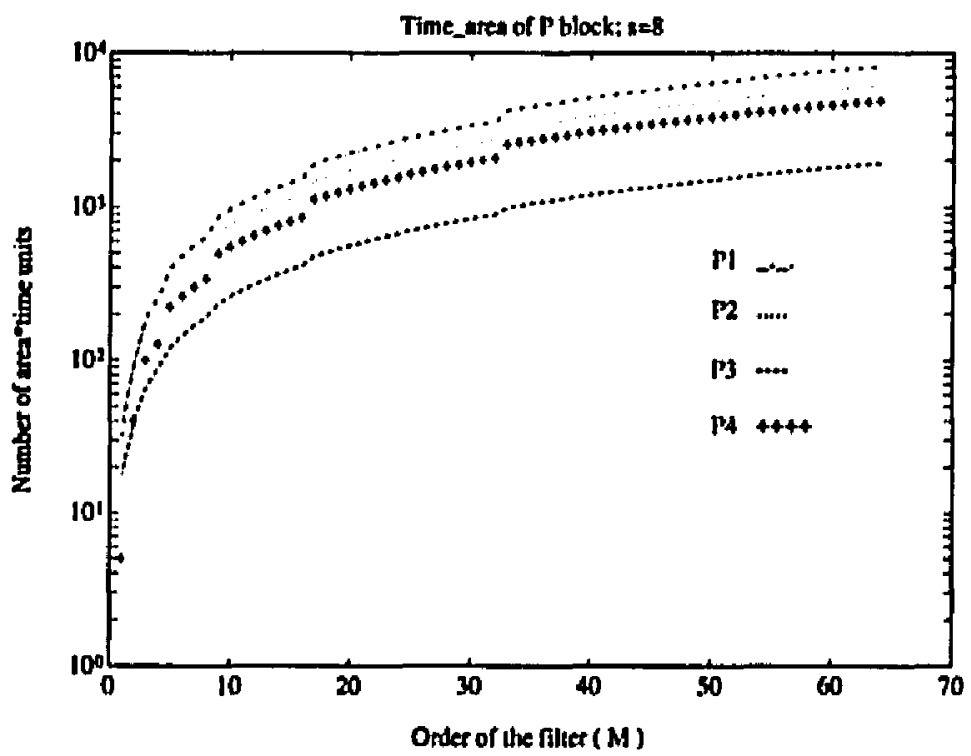

(c)

Assign $M$ multipliers to each row to calculate $q(r)$ 's as

$$
q(r)=f(r) * \hat{x}(n+s i) \text { where } s i=\left\lfloor\log _{2} r\right\rfloor
$$

Then, $\Delta w(n+s)$ can be obtained from $q(r)$ 's

$$
\Delta w(n+s)=\sum_{r=1}^{2 n-1} q(r)
$$

To implement Eq. 5.17 total of $2^{4}-s-1$ multipliers are needed with $\left[\log _{2} s\right\rceil T_{\text {mult }}$ delay time. Equation 5.18 needs $M\left(2^{\circ}-1\right)$ multipliers with $T_{\text {mult }}$ delay and Eq. 5.19 requires $M\left(2^{\prime}-1\right)$ adders with $s T_{\text {add }}$ delay. Total time required to complete the above tasks is 


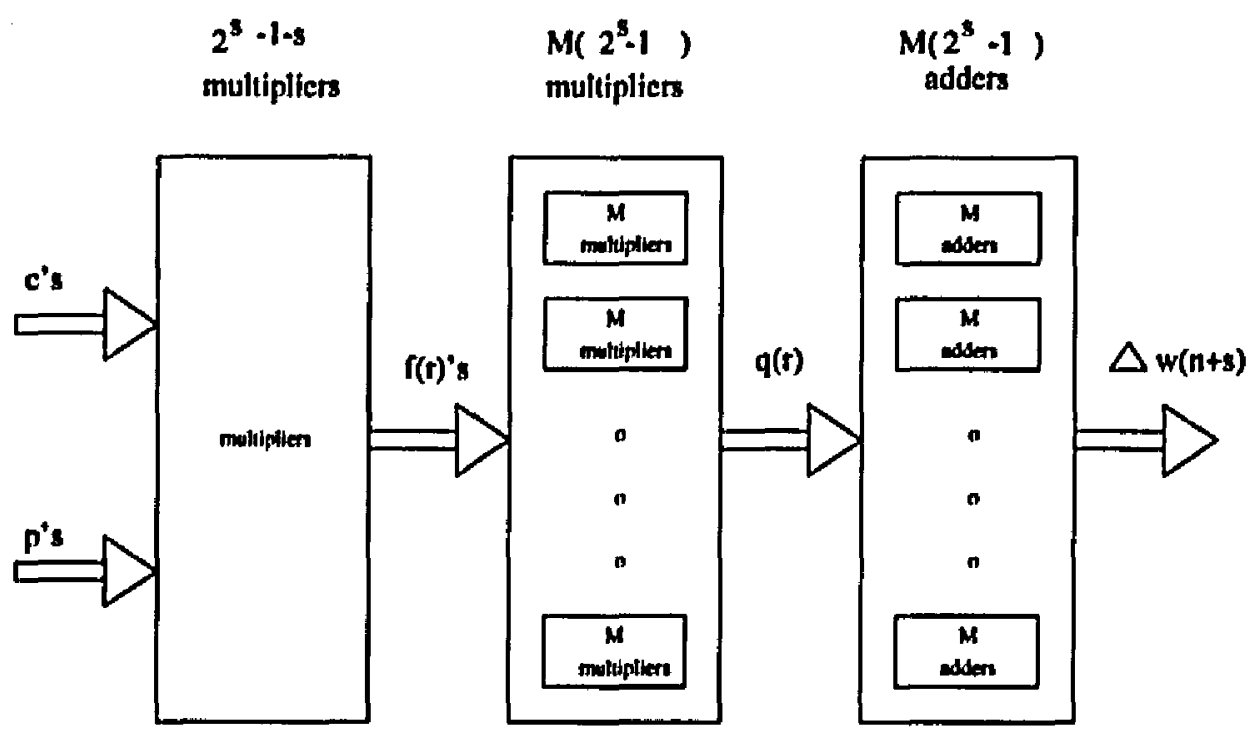

Figure 31: Block diagram of the D1 method

$$
T_{D 1}=\left(\left\lceil\log _{2} s\right\rceil+1\right) T_{\text {mule }}+s T_{\text {add }}
$$

Fig. 31 shows the block diagram to implement D1 method.

The second approach to calculate $\Delta w(n+s)$ is to compute all $f(r)$ 's , Eq. 5.17. Then add each group of $d(r)$ 's to obtain $g(s i)$, the scalar factor for each $\hat{\mathbf{x}}(n+s i)$ vector

$$
g(s i)=\sum_{r=2^{+i}}^{2^{\prime+1}+1-1} f(r) \text { for } s i=0,1, \ldots, s-1 .
$$

Then multiply $g(s i)$ to its corresponding vector, $\hat{x}(n+s i)$, to get $\mathrm{dwv}(s i)$ vector

$$
\mathrm{dwv}(s i)=g(s i) \hat{\mathbf{x}}(n+s i)
$$

Finally the summation of all $\operatorname{dwv}(s i), s i=0,1, \ldots s-1$, vectors gives $\Delta w(n+s)$

$$
\Delta w(n+s)=\sum_{s i=0}^{-1} \mathrm{dwv}(s i)
$$




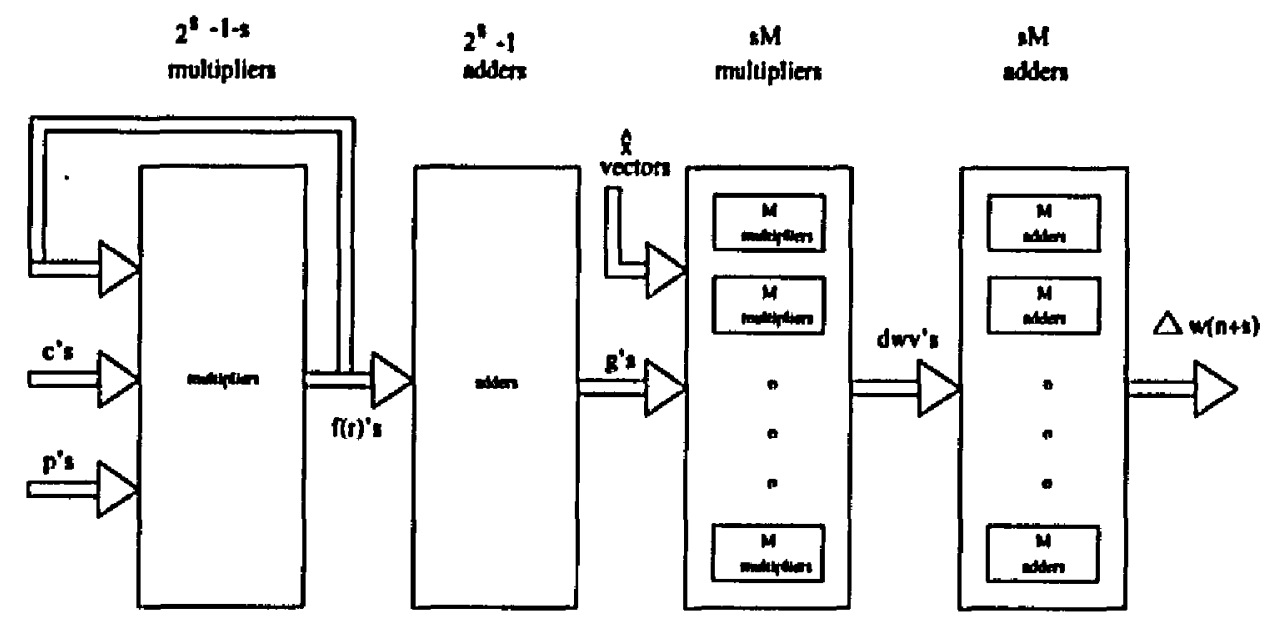

Figure 32: Block diagram of the D2 method

Fig. 32 shows the block diagram for D1 method.

Eq. 5.20 needs $2^{a-1}$ adders and it takes $(s-1) T_{\text {add }}$ time to calculate $g(s-1)$, the most time consuming $g$. Eq. 5.21 requires $s M$ multipliers with $T_{\text {mult }}$ delay time. The last stage of this method, Eq. 5.22 needs $s M$ adders and consumes $\left\lceil\log _{2} s\right\rceil T_{\text {add }}$ to calculate $\Delta w(n+s)$. Total time delay in this method is

$$
T_{D 2}=\left(\left\lceil\log _{2} s\right\rceil+1\right) T_{\text {mult }}+\left(s-1+\left\lceil\log _{2} s\right\rceil\right) T_{\text {add }}
$$

Using multiplexors to route inputs to adders and multipliers, one can reuse these units in different iterations of calculating $\Delta w(n+s)$. This is the third method for $\Delta$ block, D3, which is similar to the second method. However in the D3 method, the circuit is implemented with less adders and multipliers. Fig. 33 depicts the block diagram to implement D3 method.

$2^{2-1}$ multipliers are needed to execute Eq. 5.17 with the time delay of $\left(\left[\log _{2} s\right]\right)\left(T_{\text {mult }}+\right.$ $\left.T_{\text {murs }}\right)$, where $T_{\text {mur }}$ is the delay time of a 1-out-of-s multiplexor. Eq. 5.20 is imple- 


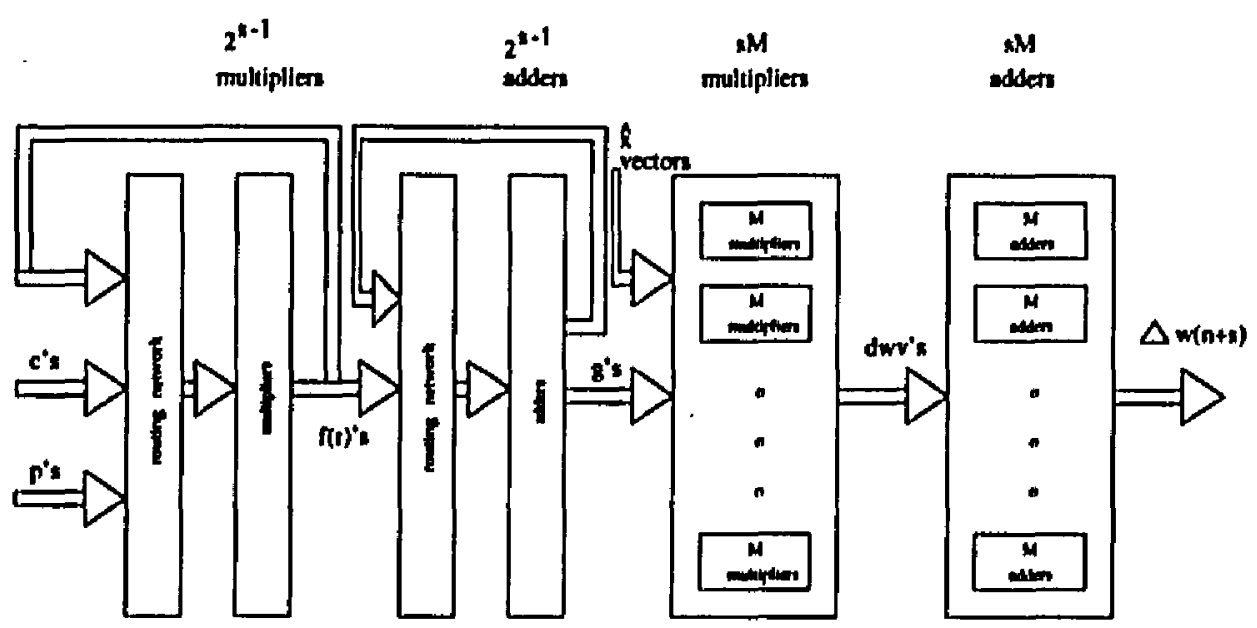

Figure 33: Block diagram of the D3 method

mented using $2^{s-1}$ adders with a time delay of $(s-1)\left(T_{a d d}+T_{\text {mur- }}\right)$. The last two steps of the third method are the same as the last two steps of the second method, Eq's 5.21 and 5.22. Therefore the total time of this method is

$$
T_{b_{3}}=\left(\left[\log _{2} s\right\rceil+1\right) T_{\text {mult }}+\left(s-1+\left\lceil\log _{2} s\right\rceil\right) T_{\text {add }}+\left(s-1+\left\lceil\log _{2} s\right\rceil\right) T_{\text {mux }} \text {. }
$$

In the next architecture of the D block, D4, one can use the overlapping time in the exccution of four steps of Eq's 5.17, 5.20, 5.21, and 5.22 to reduce the total delay time. In this method, after each multiplication in Eq. 5.17 some of $f(r)$ 's are ready to be fed into the adders of Eq. 5.20. In the same way when a group of adders for any si, si $=0,1, \ldots s-1$, finishes the calculation of any $g(s i)$, it can pass this $g(s i)$ value to the multipliers of Eq. 5.21. And when any $d w v(s i)$ is ready, it can be added to $\Delta w(n+s)$ accumulator without waiting for other dwv(si)'s. Fig. 34 shows the block diagram for D4 method.

Table 7 shows the number of multipliers and adders needed at each iteration and total number of iterations required to complete calculating $\Delta w(n+s)$. In this table, it is assumed 


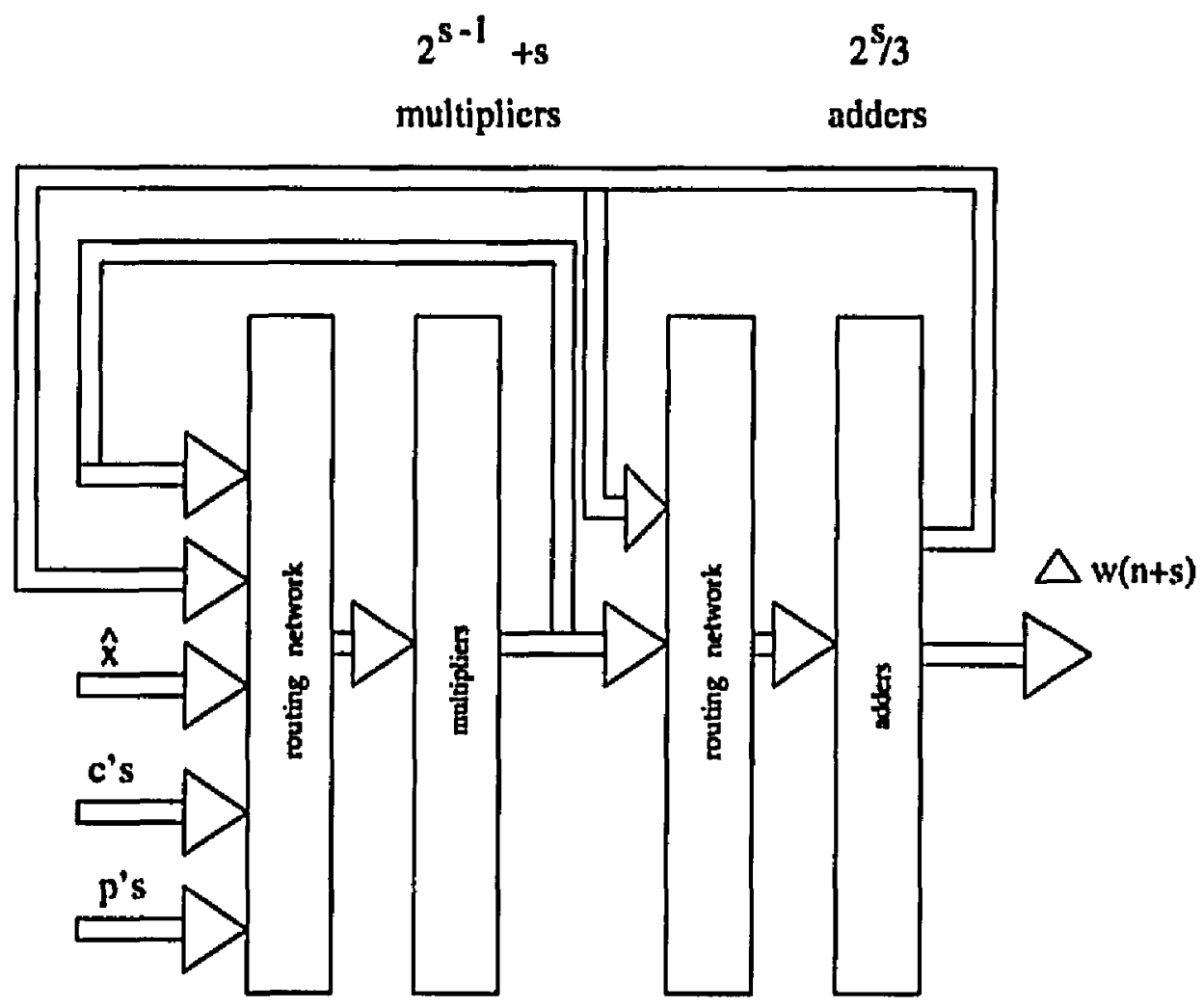

Figure 34: Block diagram of the D4 method

that $T_{\text {unit }}=T_{\text {mull }}=T_{\text {add }} \gg T_{\text {mux_s }}$ and time of an iteration is the same as $T_{\text {unit }}$.

Considering the regularity of $\Delta$ block another method to implement this block, called D5, is visible. In this method $g(s i)$ 's, the scalar factor for each $\hat{\mathbf{x}}(n+s i)$ vector, can be obtained recursively. In this way a huge saving in the number of units can be achieved, with some impact on the delay time of the filter. In D5 method, first set $g(0)=p(0)$. Then the rest of $g(s i)$ 's are

$$
g(s i)=p(s i)+\sum_{k=0}^{s i-1} c(s i, k) g(k) \quad \text { for } \quad s i=1, \ldots, s-1 .
$$

A systolic structured as shown in figure 35 calculates the $g(s i)$ for $s i=1,2, . . s-1$. 
Table 7: Required multipliers and adders and number of iterations, $T_{D 4}$, to calculate $\Delta \mathrm{w}(n+s)$ using $\mathrm{D} 4$ method.

\begin{tabular}{|c|c|c|c|c|c|c|c|c|c|c|c|c|c|}
\hline \multirow{2}{*}{$\begin{array}{c}\text { Siep } \\
\text { size } \\
\text {. }\end{array}$} & \multirow[b]{2}{*}{ Unit } & \multirow[b]{2}{*}{1} & \multirow[b]{2}{*}{2} & \multirow[b]{2}{*}{3} & \multicolumn{4}{|c|}{ Iterations $\left(T_{D 4}\right)$} & \multirow[b]{2}{*}{8} & \multirow[b]{2}{*}{9} & \multirow[b]{2}{*}{10} & \multirow[b]{2}{*}{ it } & \multirow[b]{2}{*}{12} \\
\hline & & & & & 4 & & & 7 & & & & & \\
\hline $\begin{array}{l}2 \\
2\end{array}$ & $\begin{array}{l}\text { Mult } \\
\text { Add }\end{array}$ & $\begin{array}{l}1 \\
0\end{array}$ & 1 & & & & & & & & & & \\
\hline 3 & Mult & 3 & 1 & & & & & & & & & & \\
\hline 3 & Add & 0 & 2 & $\mathbf{I}$ & 1 & & & & & & & & \\
\hline $\begin{array}{l}4 \\
4\end{array}$ & $\begin{array}{l}\text { Mult } \\
\text { Add }\end{array}$ & $\begin{array}{l}7 \\
0\end{array}$ & 5 & 4 & 2 & 1 & & & & & & & \\
\hline 5 & Mult & 15 & 15 & 1 & & & & & & & & & \\
\hline 5 & Add & 0 & 6 & 10 & 6 & 3 & $\mathbf{I}$ & & & & & & \\
\hline $\begin{array}{l}6 \\
6\end{array}$ & $\begin{array}{l}\text { Mult } \\
\text { Add }\end{array}$ & $\begin{array}{r}31 \\
0\end{array}$ & $\begin{array}{r}35 \\
9\end{array}$ & $\begin{array}{r}7 \\
21\end{array}$ & 14 & 8 & 3 & I & & & & & \\
\hline $\begin{array}{l}7 \\
7\end{array}$ & $\begin{array}{l}\text { Mult } \\
\text { Add }\end{array}$ & $\begin{array}{r}63 \\
0\end{array}$ & $\begin{array}{l}71 \\
12\end{array}$ & $\begin{array}{l}29 \\
40\end{array}$ & 35 & 18 & B & 4 & 2 & 1 & & & \\
\hline $\begin{array}{l}8 \\
8\end{array}$ & Mult & 127 & $\begin{array}{r}135 \\
6\end{array}$ & 93 & 87 & 42 & 19 & 10 & 5 & 2 & 1 & & \\
\hline $\begin{array}{l}9 \\
9\end{array}$ & $\begin{array}{l}\text { Mult } \\
\text { Add }\end{array}$ & $\begin{array}{r}255 \\
0\end{array}$ & $\begin{array}{r}255 \\
20\end{array}$ & $\begin{array}{l}235 \\
114\end{array}$ & $\begin{array}{r}1 \\
186\end{array}$ & 92 & 46 & 23 & 12 & $\mathbf{5}$ & 3 & I & \\
\hline $\begin{array}{l}10 \\
10 \\
\end{array}$ & $\begin{array}{l}\text { Mult } \\
\text { Add }\end{array}$ & $\begin{array}{r}511 \\
0\end{array}$ & $\begin{array}{r}495 \\
25\end{array}$ & $\begin{array}{l}627 \\
176 \\
\end{array}$ & $\begin{array}{r}11 \\
403\end{array}$ & 206 & 103 & 52 & 25 & 12 & 7 & 3 & 1 \\
\hline
\end{tabular}

Each element of this structure, Fig. 35(a) and 35(b), needs one multiplier and one adder with a total time delay of $T_{\text {mult }}+T_{\text {add }}=2 T_{\text {unit }}$.

Since in this method each $g(s i)$ is evaluated every two $T_{\text {unit }}$ Eq's 5.21 can be implemented using $M$ multipliers instend of $s M$ multipliers as was proposed in D2 and D3. In the same way, Eq. 5.22 can be implemented using $M$ adders instead of $s M$ adders. Total time for $\Delta$ block using this approach is

$$
T_{D s}=(2 s+1)\left(T_{m u l t}+T_{\text {add }}\right) \text {. }
$$

Table 8 shows the area and time required to implement all five methods for the $\Delta$ block. 


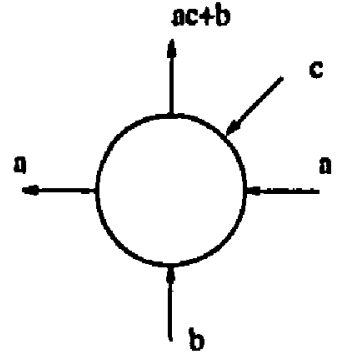

(b)

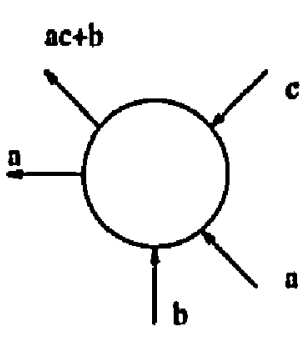

(a) Diagond Dement

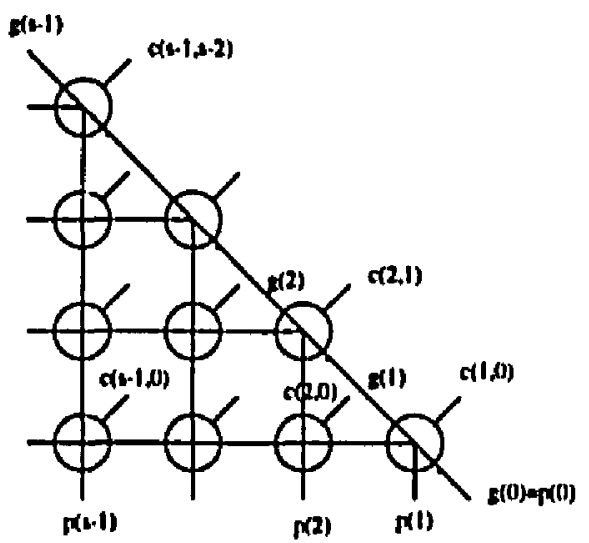

(c) Syulk Amy

Figure 35: G structure to calculate $g($ si)'s recursively; (a) A Diagonal Element; (b) A Non_Diagonal Element; (c) Overall Recursive Structure to calculate $g(s i)$ 's 
Table 8: Comparison of the five methods to implement the $\Delta$ block

\begin{tabular}{|c|c|c|c|c|c|c|}
\hline $\begin{array}{l}\text { Methed" } \\
\text { neme }\end{array}$ & Requitrod mulupilikn & $\begin{array}{l}\text { Denter of tritu } \\
\text { Requited edent }\end{array}$ & 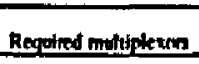 & Mulliplicattkm & 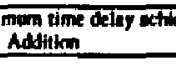 & metilistexint: \\
\hline DI & $2^{2}-1-1+M\left(2^{\prime-1}\right)$ & $M\left(2^{-t}\right)$ & o & $\mid \ln 12 \cdot 1+1$ & $1-1$ & 0 \\
\hline D2 & $2 x=-1+x$ & $2^{2-1}+n$ & 0 & $1+\log _{2}+1$ & $-1+\mid \ln _{12} \cdot 1$ & n \\
\hline D3 & $2^{\prime-1}+0 M$ & $2^{1-1}+N$ & $2^{x-1}+o M$ & $\operatorname{Ant2} 1$ & $\cdot-1+\ln _{32} \cdot 1$ & $-1+\left\{n_{x_{2}}+1\right]$ \\
\hline D4 & $2^{x-1}+c$ & 4 & 24 & $\left\lfloor\log _{2}(\cdot+1)\right]$ & $0-1$ & - \\
\hline Ds & $\frac{n(e-1)}{2}+M$ & $\frac{(0-1)}{2}+M$ & $1+M$ & $=+1$ & $\cdot+1$ & 1 \\
\hline
\end{tabular}

Consider the same assumptions made in presentation of the C block methods, 5.10, 5.11, and 5.12. Fig. 36 shows the delay time, required area, and timearea performance of the different methods of implementing the $\Delta$ block versus the step size $s$. In this figure the order of the filter is constant and equal 128.

Fig. 37 depicts the time, area, and time_area performance of five $\Delta$ methods with respect to order of the filter for constant step size $s=8$.

As Fig. 36(a) and 37(a) show DI and D4 have the best time performance, with respect to either parallel step size or order of the filier. However the area of D4 for small $s$ and D5 for large $s$ leads the others, Fig. 36(b) and 37(b). In the time_area performance, Fig. 36(c) and 37(c), the competition is between D4 and D5. Therefore these two methods are used in investigation of the over all performance of the PBS_LMS algorithm.

$$
\begin{aligned}
& \text { Adding } \Delta w(n+s) \text { to } w(n) \text { produces } w(n+s) \\
& w(n+s)=\Delta w(n+s)+w(n)
\end{aligned}
$$

This summation needs $M$ adders with $T_{\text {add }}$ time delay. 


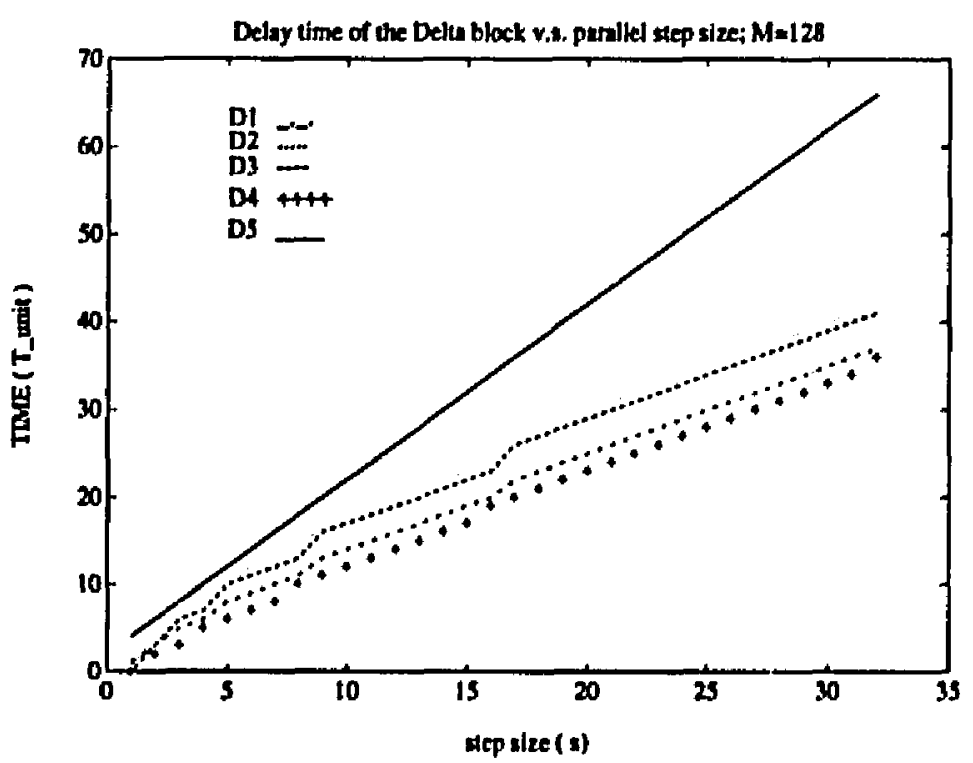

(a)

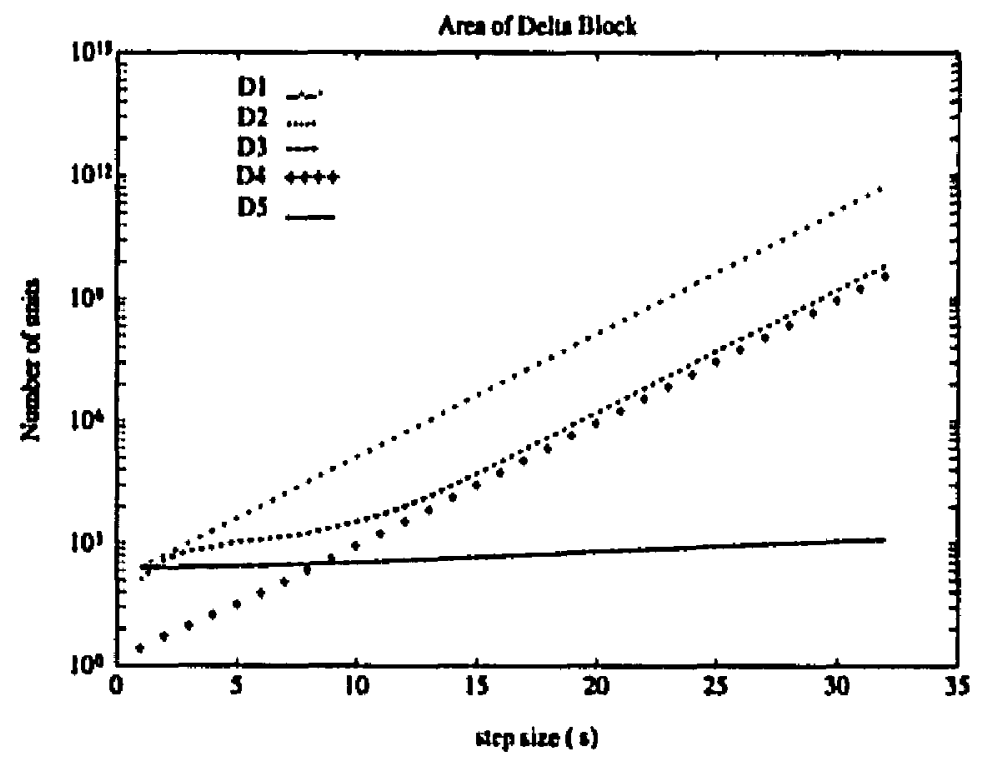

(b)

Figure 36: Comparison among the different $\Delta$ block's methods for constant order of the filter, $M=128$. (a) Total time delay for the $\Delta$ block versus step size $s$. (b) Total area required for the $\Delta$ block versus step size $s$. (c) Time area presentation of different D's versus step size $s$ 
Fig. 36 (continued)

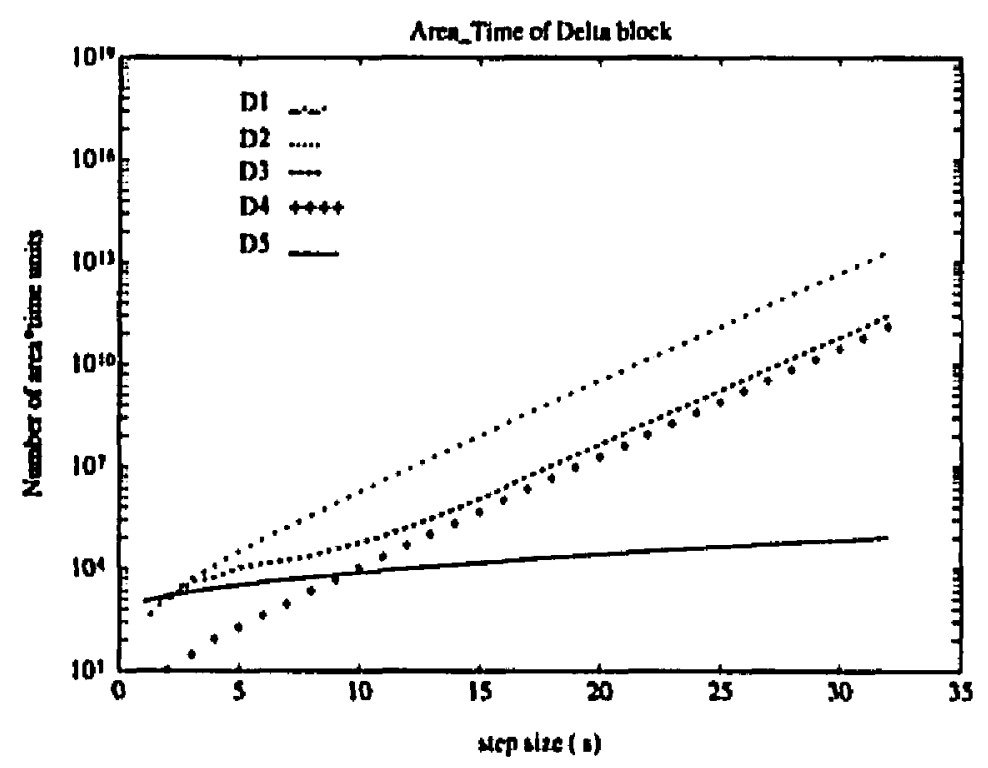

(c) 


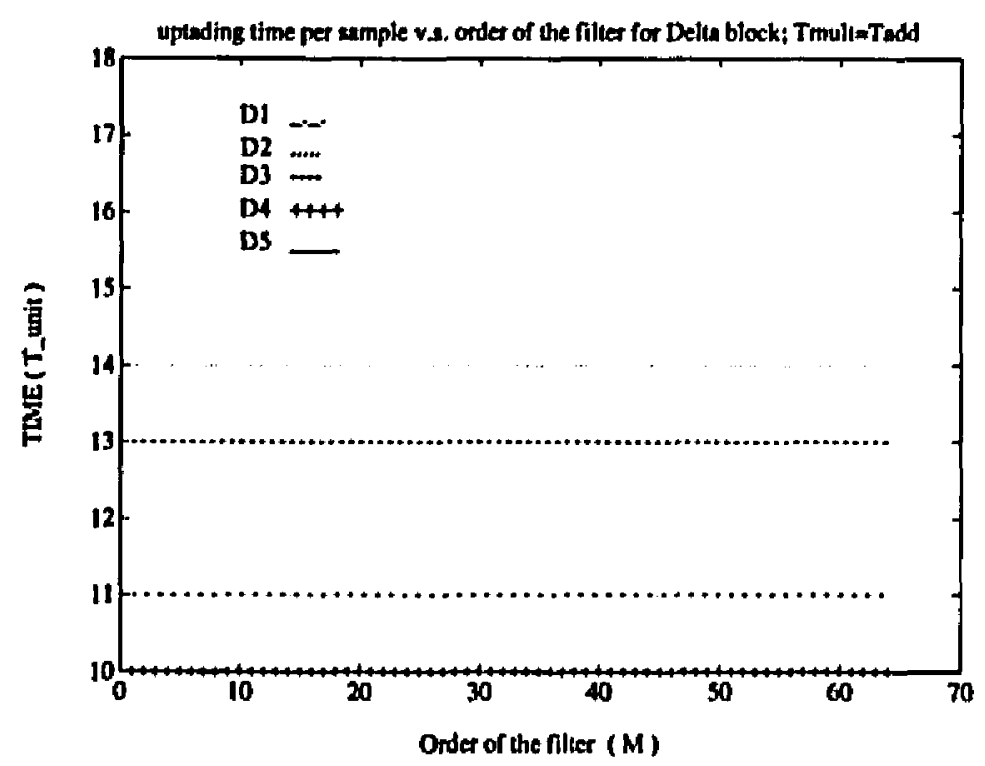

(a)

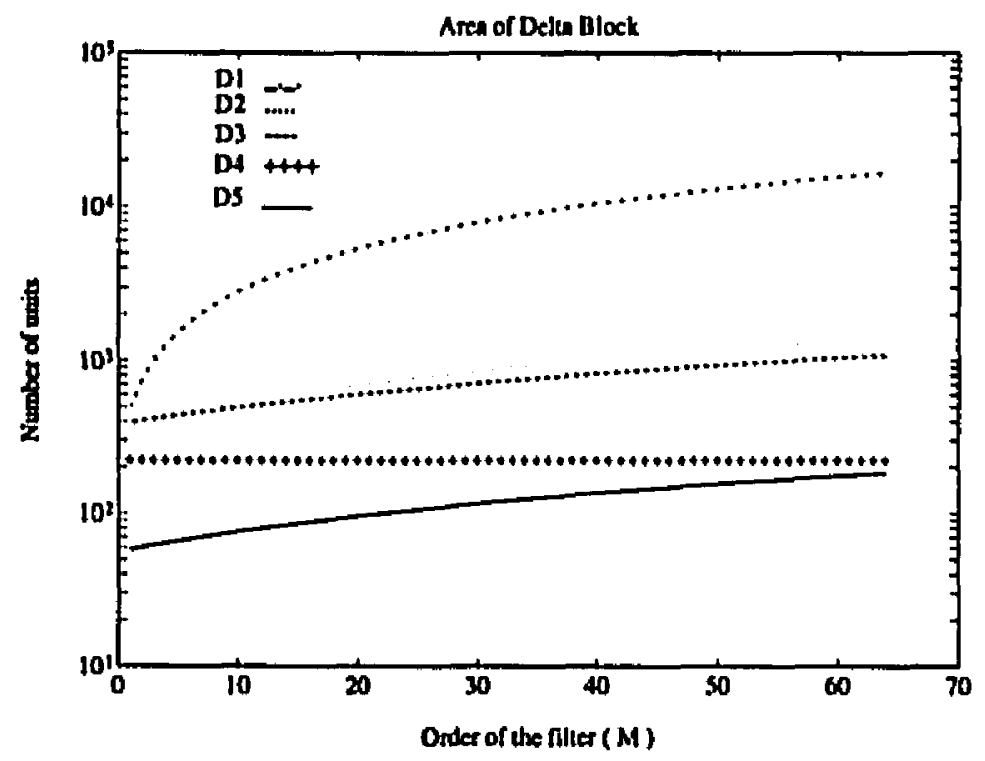

(b)

Figure 37: Comparison among the different $\Delta$ block's methods for constant step size, $s=8$. (a) Total time delay for the $\Delta$ block versus order of the filter, (b) Total area required for the $\Delta$ block versus order of the filter, (c) Time_area presentation of different D's versus order of the filter 
Fig. 37 (continued)

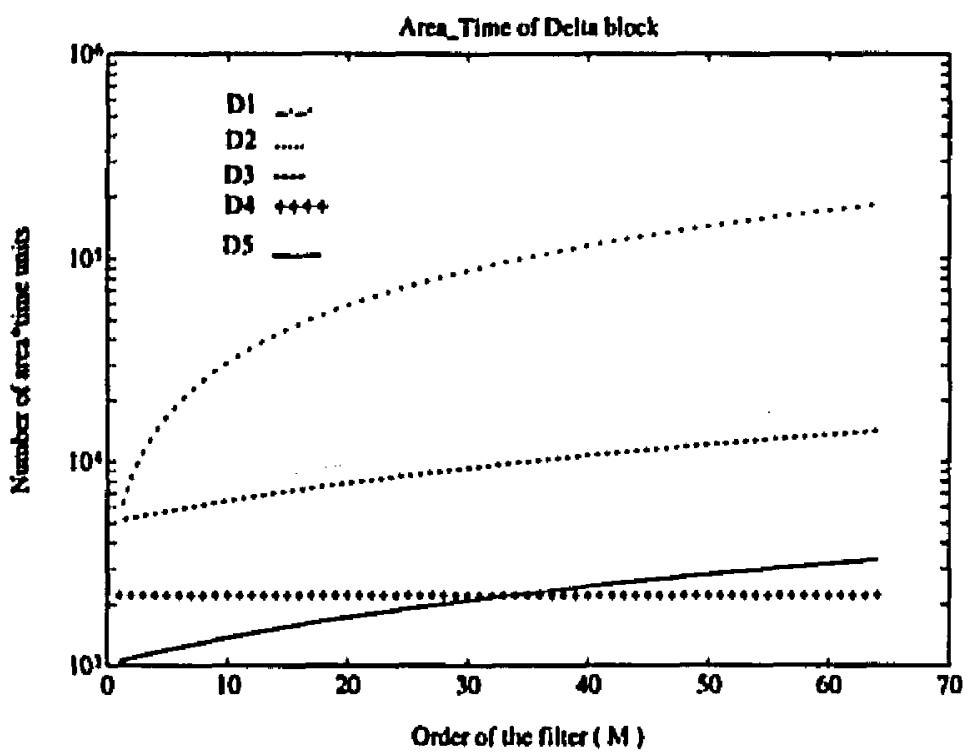

Fig. 37(c) 


\subsection{Evaluation of overall PBS.LMS performance using different P's, C's, and D's methods:}

As mentioned, the calculation of the $\mathrm{C}$ and $\mathrm{P}$ blocks is independent and can occur in parallel. Therefore, the time required to calculate the tap weights with $s$ step update is the maximum of $T_{C}$ and $T_{P}$ plus $T_{\Delta}$ plus $T_{\Sigma} . T_{\Sigma}$ is the time to add $\Delta w(n+s)$ to $w(n)$ to obtain $w(n+s)$. Thus

$$
T_{\text {total-PBSLMS }}=\max \left(T_{C}, T_{P}\right)+T_{\Delta}+T_{\Sigma}
$$

The average time delay per each input sample for parallel binary structured LMS, $T_{P B S} \perp M S$, is

$$
T_{P B S \perp M S}=\frac{T_{\text {total-PBS.LMIS }}}{s}
$$

Consider eight possible structures for PBS.LMS using all combinations of C2 or C4, P2 or P3, and D4 or D5. Followings are the definitions of these cight combinations, called PBS_k, for $k=1,2, \ldots$ 8. For example PBS_l is a PBS $\perp M S$ adaptive filter in which $C 2$ is implemented in the $\mathrm{C}$ block, $\mathrm{P} 2$ is implemented in the P block, and D4 is implemented in the $\Delta$ block. The architectures are

$$
\begin{aligned}
& \text { PBS.I } \Longleftarrow\{C 2, \text { P2, D4 }\} \\
& \text { PBS_2 } \Longleftarrow\{C 2, \text { P2, D5 }\} \\
& \text { PBS_3 }=\{C 2, \text { P3, D4 }\} \\
& \text { PBS_4 } \Longleftarrow\{\text { C2, P3, D5 }\} \\
& \text { PBS } 5 \Longleftarrow\{\text { C5, P2, D4 }\} \\
& \text { PBS.6 } \Longleftarrow\{\text { C5, P2, D5 }\}
\end{aligned}
$$




\section{PBS_7 $\Longleftarrow\{C 5$, P3, D4 $\}$ \\ PBS.8 $\Longleftarrow\{$ C5, P3, D5 $\}$}

The delay of PBS LMS implementations are related to the time delay of the $\mathrm{C}$ block and the $\mathrm{P}$ block. Figure 38 shows the delay times of the $\mathrm{C}$ methods and the $\mathrm{P}$ methods. Fig. 38(a) and 38(b) show these delays versus parallel step size, when $M=128$ and $M=64$, respectively. Fig. 39 shows the delay times for $\mathrm{C}$ block and $\mathrm{P}$ block for different order of the filter. Figure 39(a) depicts the delays when parallel step size is $8, s=8$. Fig. 39(b) shows these delays when $s=16$.

As the figures show, in the working range of $M>7$

$$
\begin{aligned}
& T_{c 2}<T_{P 2} \\
& T_{c 2}<T_{P 3}
\end{aligned}
$$

and also

$$
\begin{aligned}
& T_{c s}>T_{P 2} \\
& T_{c s}<T_{P 3}
\end{aligned}
$$

Therefore, the average time for the PBS $k$ 's are as follows.

$$
\begin{aligned}
& T_{P B S-1}=\frac{T_{P 2}+T_{D 4}+1}{s} \\
& T_{P B S-2}=\frac{T_{P 2}+T_{D S}+1}{s} \\
& T_{P B S-3}=\frac{T_{P 3}+T_{D 4}+1}{s} \\
& T_{P B S A}=\frac{T_{P 3}+T_{D S}+1}{s} \\
& T_{P B S S}=\frac{T_{C S}+T_{D 4}+1}{s}
\end{aligned}
$$




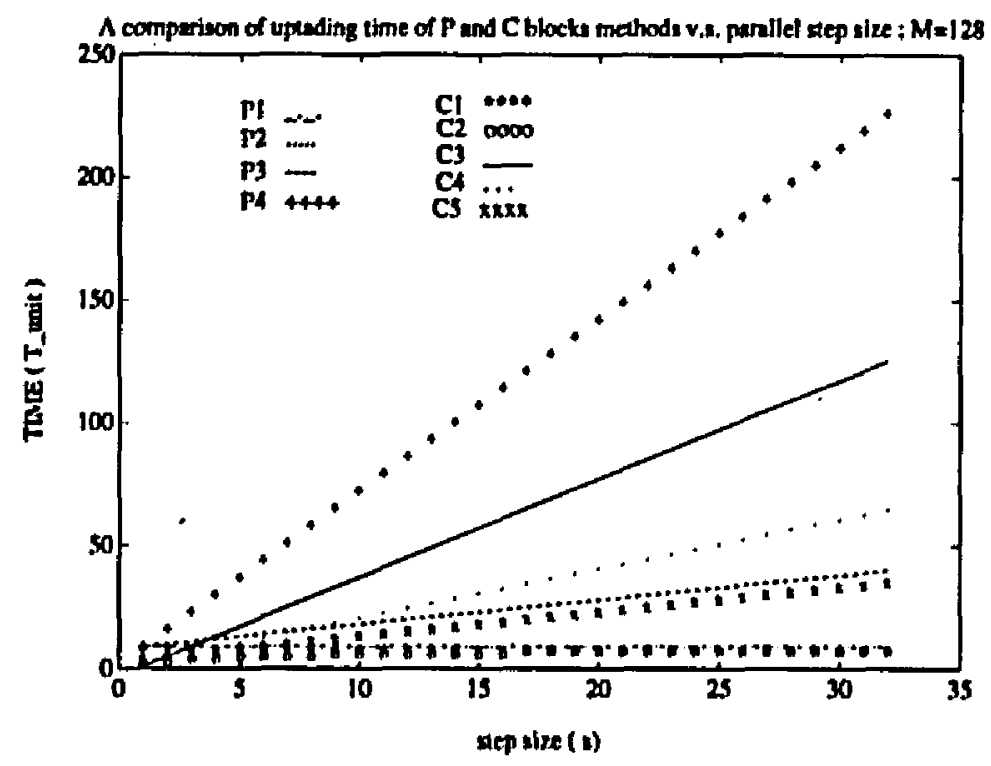

Fig. 38(a)

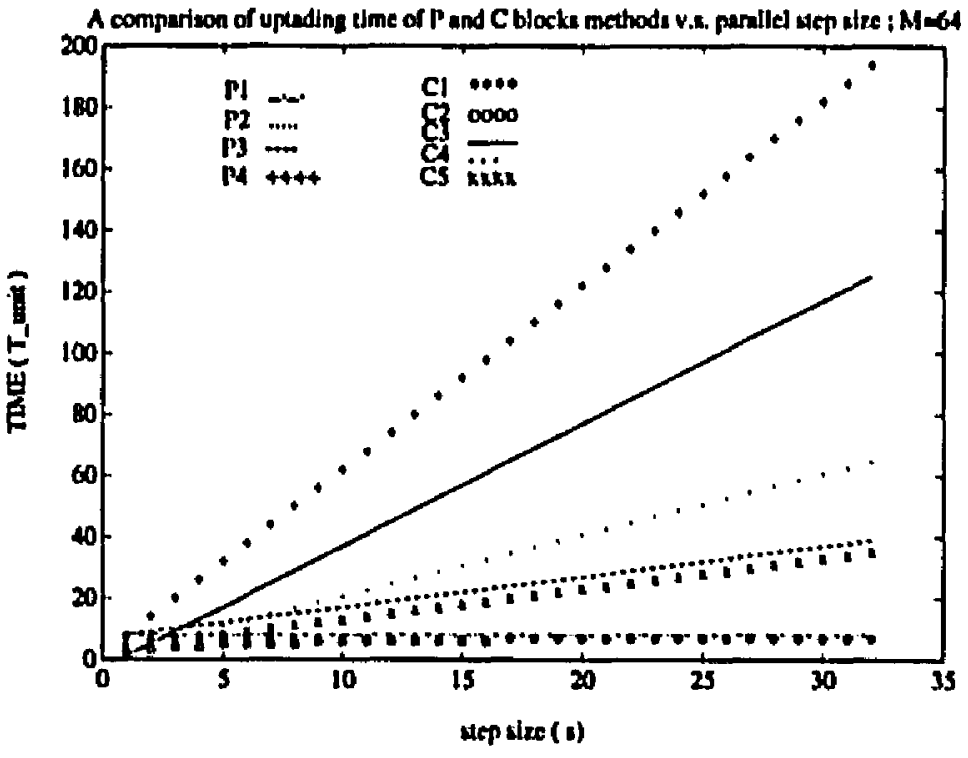

Fig. 38(b)

Figure 38: Comparison among the different $C$ and $P$ block's methods for constant order of the filter; (a) Comparison of C's and P's when $M=128$; (b) Comparison of C's and P's when $M=64$ 


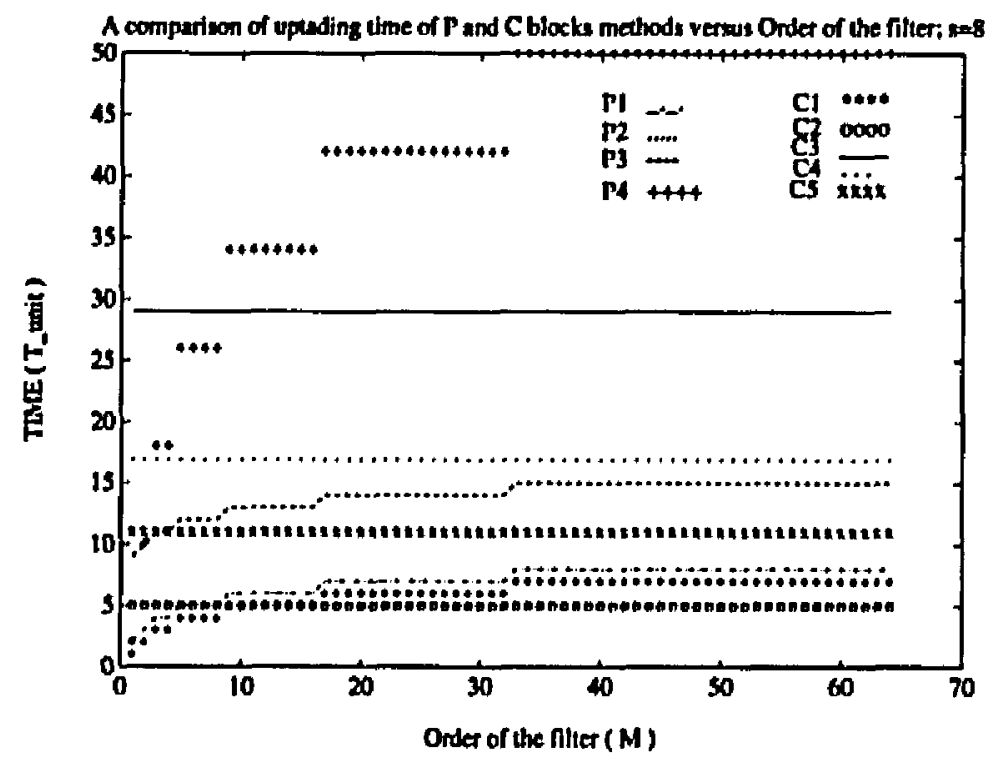

Fig. 39(a)

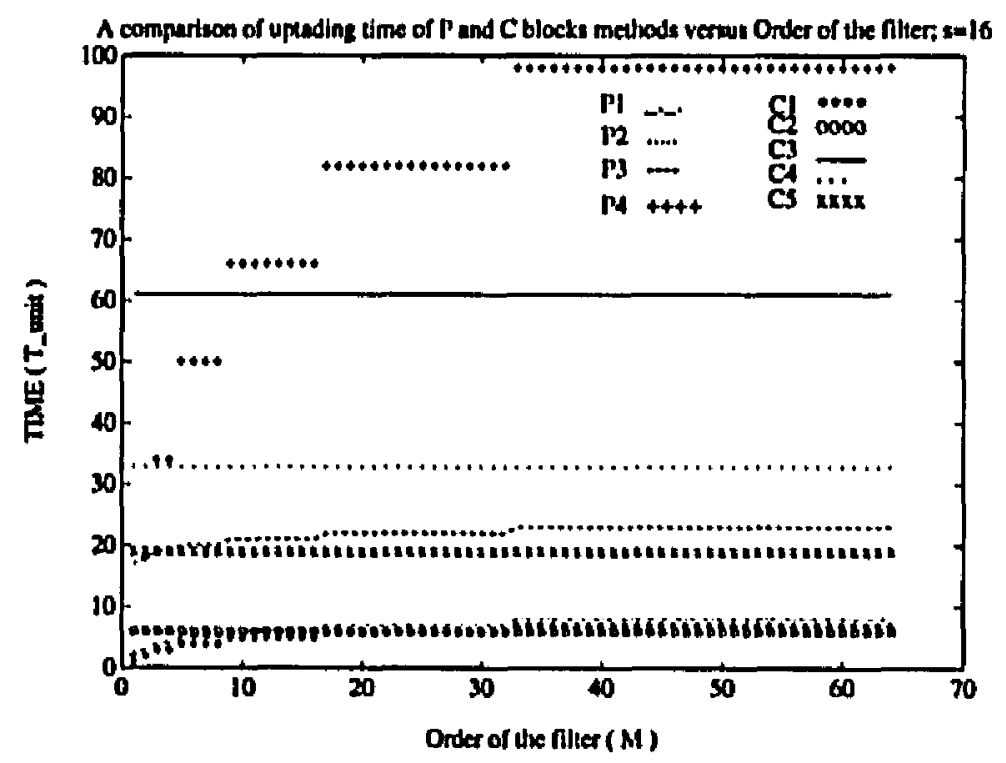

Fig. 39(b)

Figure 39: Comparison among the different $C$ and $P$ block's methods for constant order of the filter; (a) Comparison of C's and P's when $M=128$; (b) Comparison of C's and P's when $M=64$ 


$$
\begin{aligned}
& T_{P B S-G}=\frac{T_{C S}+T_{D S}+1}{s} \\
& T_{P B S-7}=\frac{T_{P 3}+T_{D A}+1}{s} \\
& T_{P B S S}=\frac{T_{P 3}+T_{D S}+1}{s}
\end{aligned}
$$

The area required to implement each of PBS $\_$methods is the sum of the area, or total number of the units, of all the blocks used in that method, plus $M$ adders for the last summation. Thus, the area of eight proposed structures are

$$
\begin{aligned}
& A_{P B S-1}=A_{P 2}+A_{D 4}+A_{C 2}+M \\
& A_{P B S-2}=A_{P 2}+A_{D S}+A_{C 2}+M \\
& A_{P B S-3}=A_{P 3}+A_{D 4}+A_{C 2}+M \\
& A_{P B S-4}=A_{P 3}+A_{D S}+A_{C 2}+M \\
& A_{P B S-S}=A_{P 2}+A_{D 4}+A_{C S}+M \\
& A_{P B S-1}=A_{P 2}+A_{D S}+A_{C S}+M \\
& A_{P B S-7}=A_{P 3}+A_{D 4}+A_{C S}+M \\
& A_{P B S A}=A_{P 3}+A_{D S}+A_{C S}+M
\end{aligned}
$$

The Time_Area performance of ench PBS_k is the product of its delay time to its area,

$$
T A_{P B S \perp}=T_{P B S \perp} * A_{P B S \perp}
$$

Figure 40 shows the performance of eight methods of PBS.LMS's versus parallel step size, for constant filter size, $M=128$. 


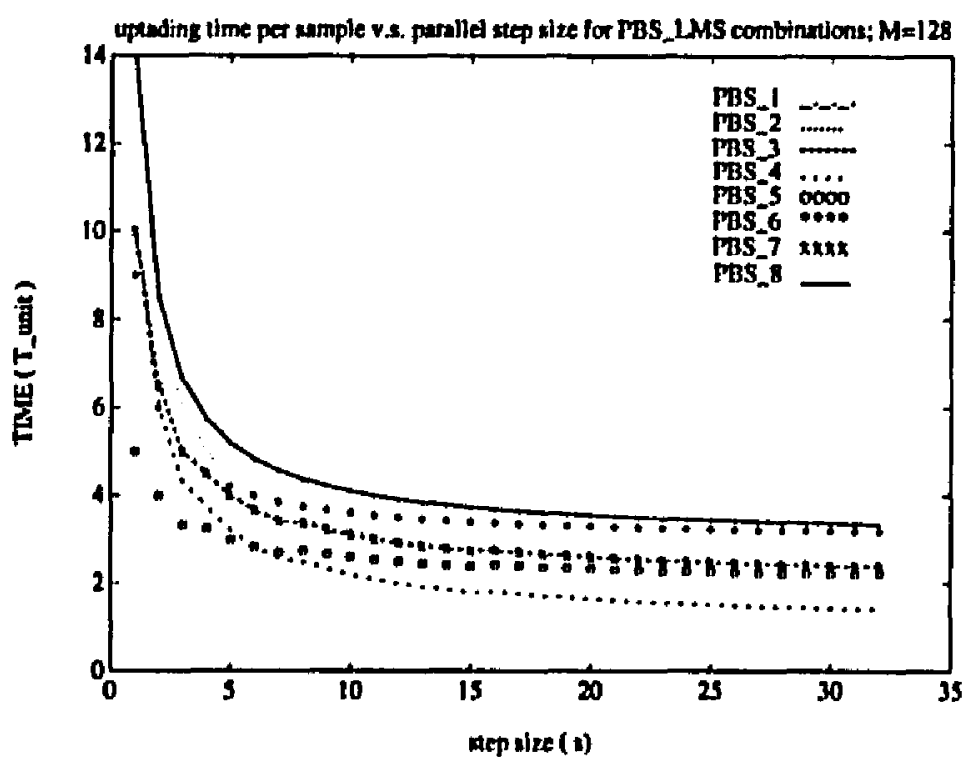

(a)

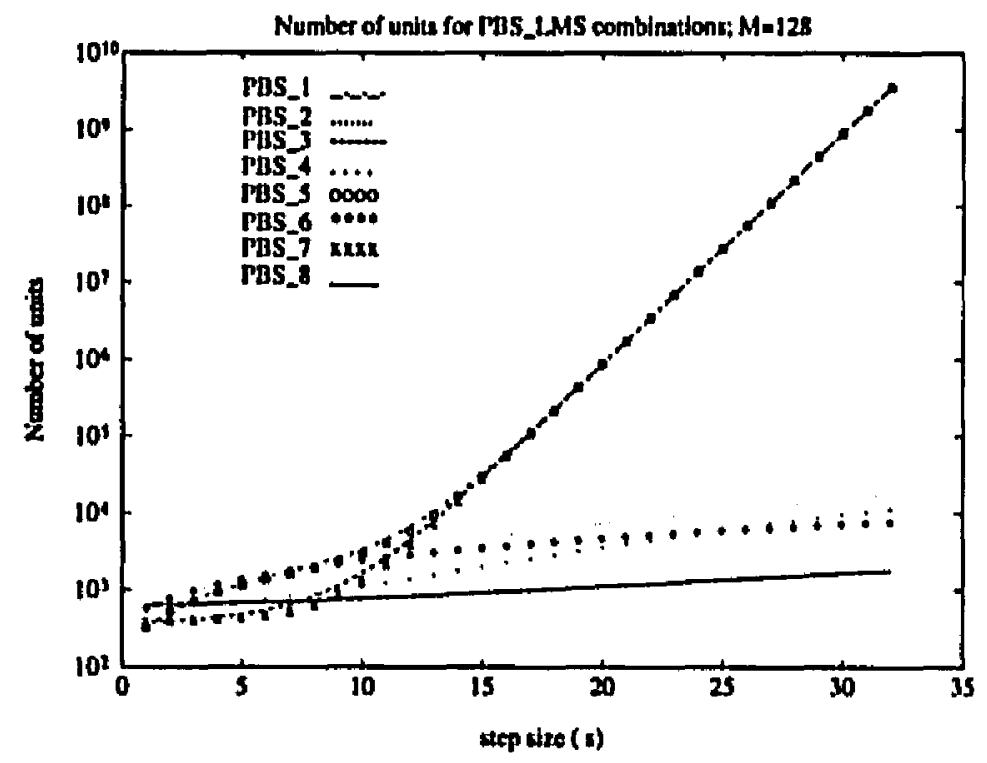

(b)

Figure 40: Comparison among the different PBS.LMS methods versus step size for constant filter's order, $M=128$. (a) Total time delay for the PBS.LMS methods versus step size; (b) Total area required for the PBS $\perp$ MS versus step size; (c) Time_area presentation of different PBS.LMS versus step size 
Fig. 40 (continued)

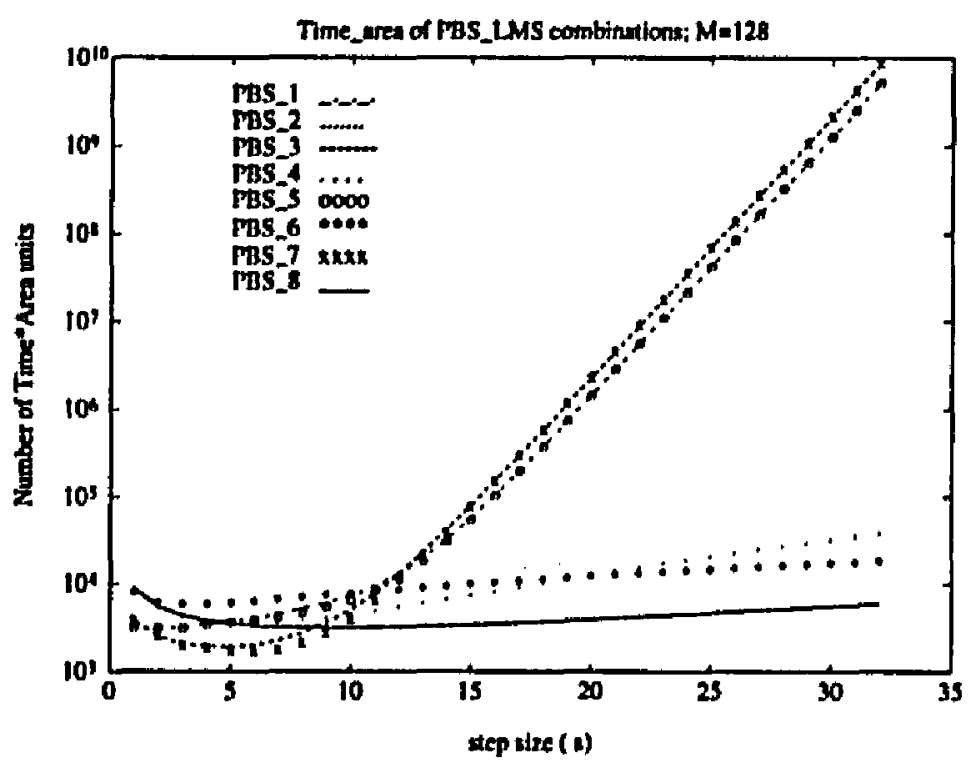

(c)

Figure 41 shows the performance of eight methods of PBS.LMS's versus order of the filter, for constant step size, $s=8$.

Fig. 40 and Fig. 41 show that for small step size $s$ PBS. 1 and PBS 5 are the two fastest architectures for PBS.LMS algorithm. However, these architectures are weak in the time_area performance. Other architectures such as PBS_7, PBS_3, and PBS_.8 are the best in the time.area performance, when step size, $s$, is less than 10. For step size $s>10$ PBS_8, PBS_4, and PBS_6 have the best time_area products. 


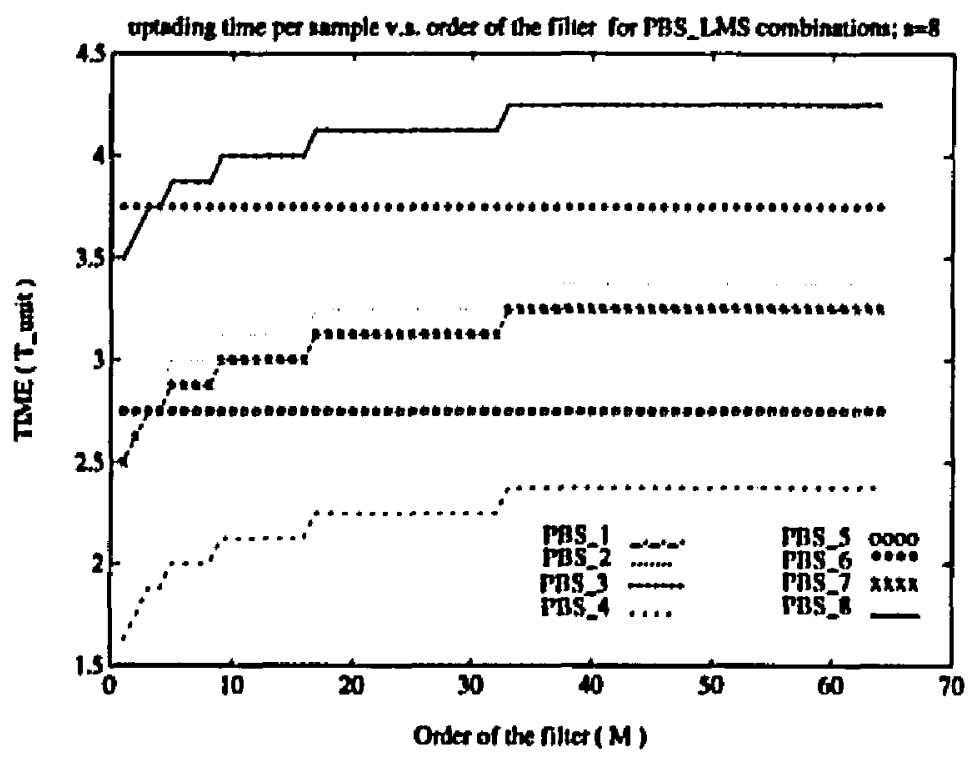

(a)

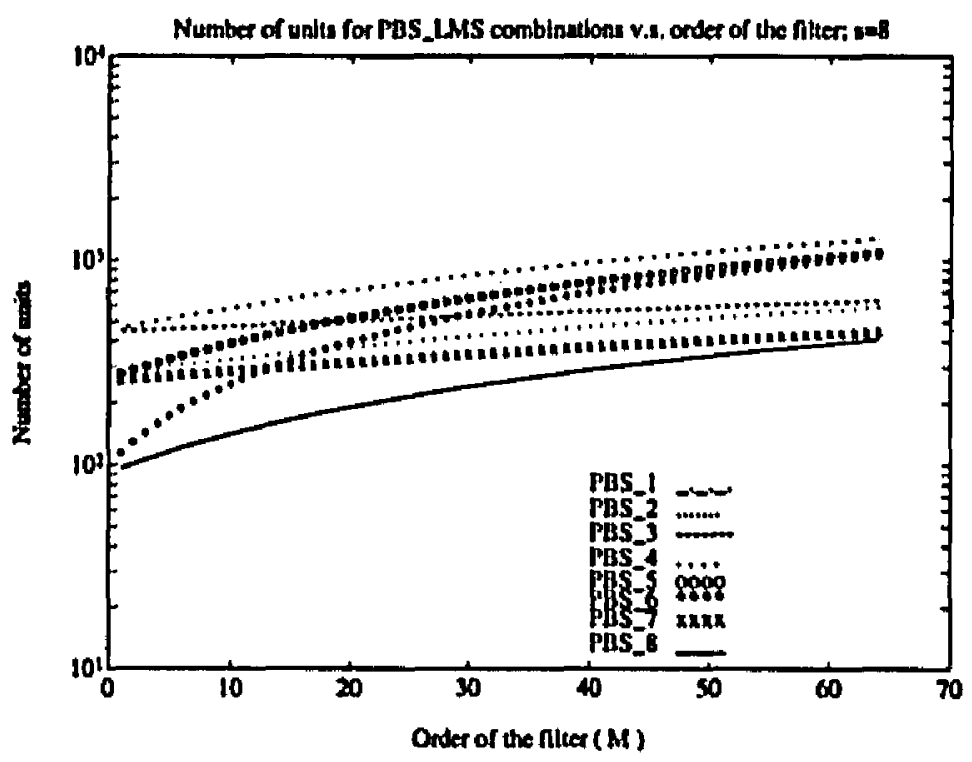

(b)

Figure 41: Comparison among the different PBSLMS methods order of the filter, for constant step size, $s=8$, (a) Total time delay for the PBS $L M S$ methods versus order of the filter; (b) Total area required for the PBS_LMS versus order of the filter; (c) Time_area presentation of different PBS.LMS versus order of the filter 
Fig. 41 (continued)

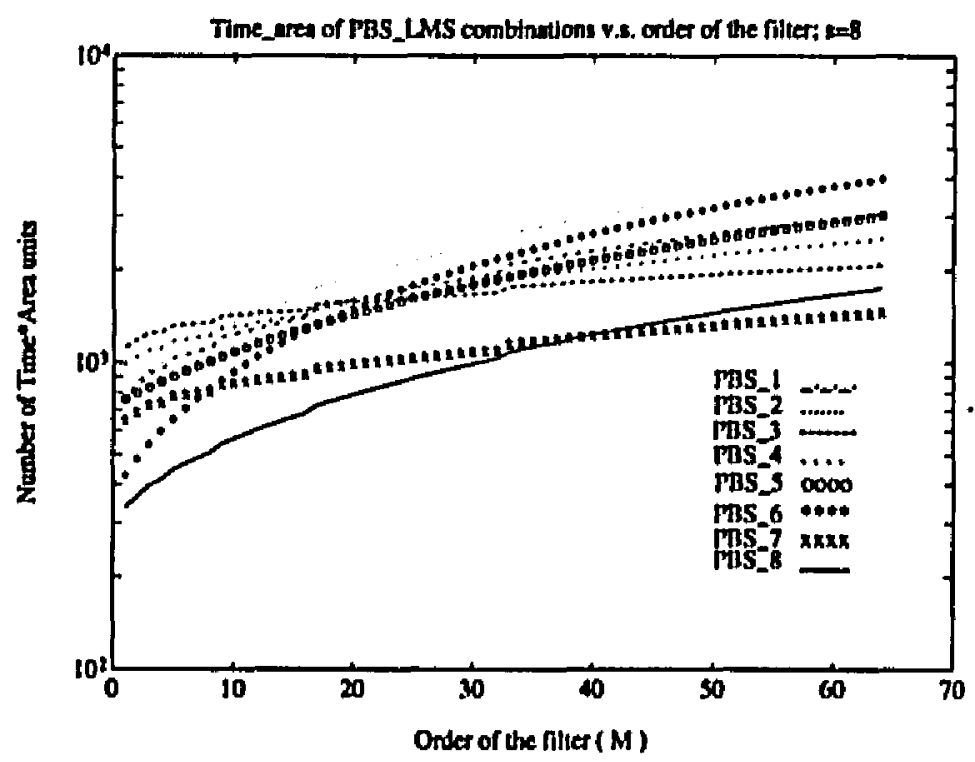

(c)

\subsection{Example, implementation of PBS_8 for $s=4$ and $M=8$}

As an example, consider a parallel binary structured adaptive filter of order $8, M=8$, with 4 step lookahead, $s=4$. To implement PBS_8 architecture, C5 architecture is implemented in the $\mathrm{C}$ block, P3 architecture is implemented in the P block, and DS architecture is implemented in the $\Delta$ block.

Fig. 42 shows the detail architecture of the $\mathrm{C}$ block in this example. The configuration of the P block is depicted in the Fig. 43. Fig. 44 shows the systolic structure to calculate the $g$ coefficients in the $\Delta$ block. The calculation of $\mathrm{dwv}(n+s)$ and the last accumulator to calculate the $w(n+s)$ is shown in Fig. 45 . 
To implement such an adaptive filter 68 arithmetic units are required. An arithmetic unit is a floating point adder or multiplier. Assume the area of each floating point adder or multiplier occupies $1750 \lambda \times 1500 \lambda$ of a die, where $\lambda$ is the scale factor and equal to one half of the technology factor size. Also, consider a total of $50 \%$ of the area is needed for routing. arithmetic units. Then, the total area of a chip, with $\lambda=0.5 \mu m$, to built this filter is

$$
\begin{aligned}
A R E A & =68(1750 \times 1500)\left(0.5 \times 10^{-6}\right)^{2} \times 2 \\
& =89.25 \mathrm{~mm}^{2}
\end{aligned}
$$

That is, this adaptive filter would require a die $9.4 \mathrm{~mm} \times 9.4 \mathrm{~mm}$.

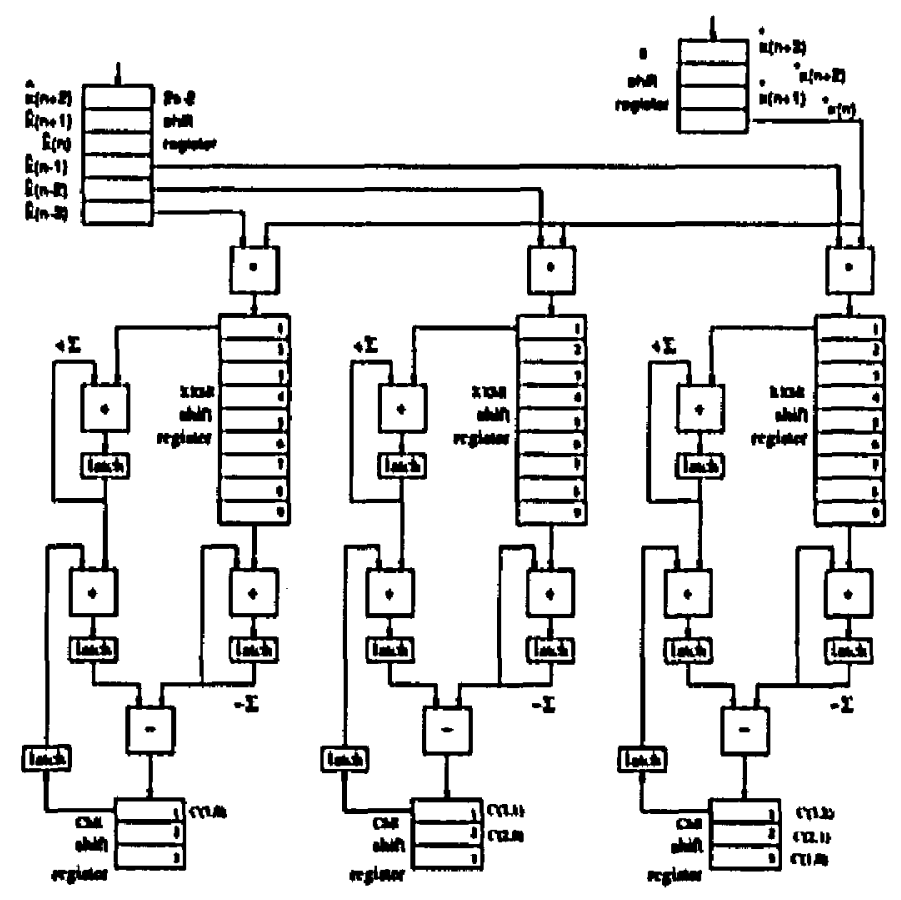

Figure 42: The configuration of the $\mathrm{C}$ block of PBS .8 architecture for an adaptive filter of order 8 and parallel step size 4. 


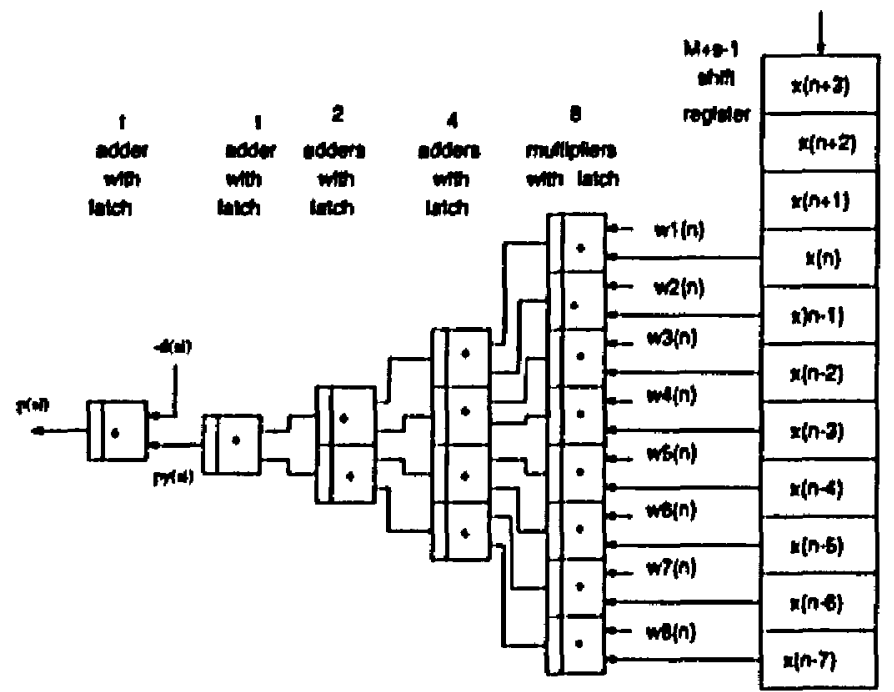

Figure 43: The configuration of the P block of PBS.8 architecture for an adaptive filter of order 8 and parallel step size 4.

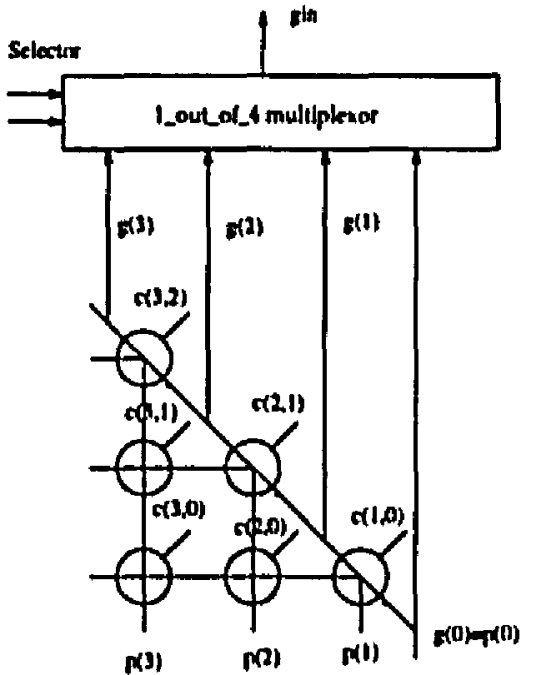

(c) Symadic Nroy

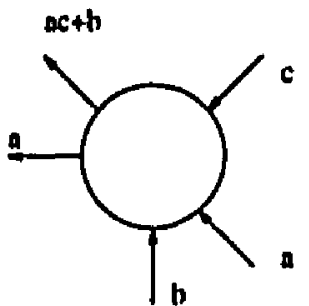

(a) Diagneal Exemens

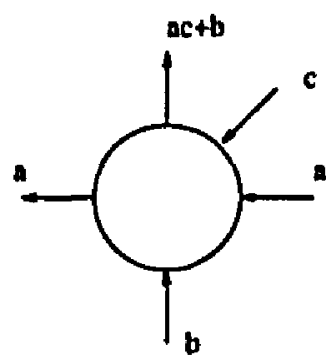

(b) Nim Diasunu Pement

Figure 44: The systolic structure to calculate $g$ coefficients in the PBS.8 architecture for an adaptive filter of order 8 and parallel step size 4. 


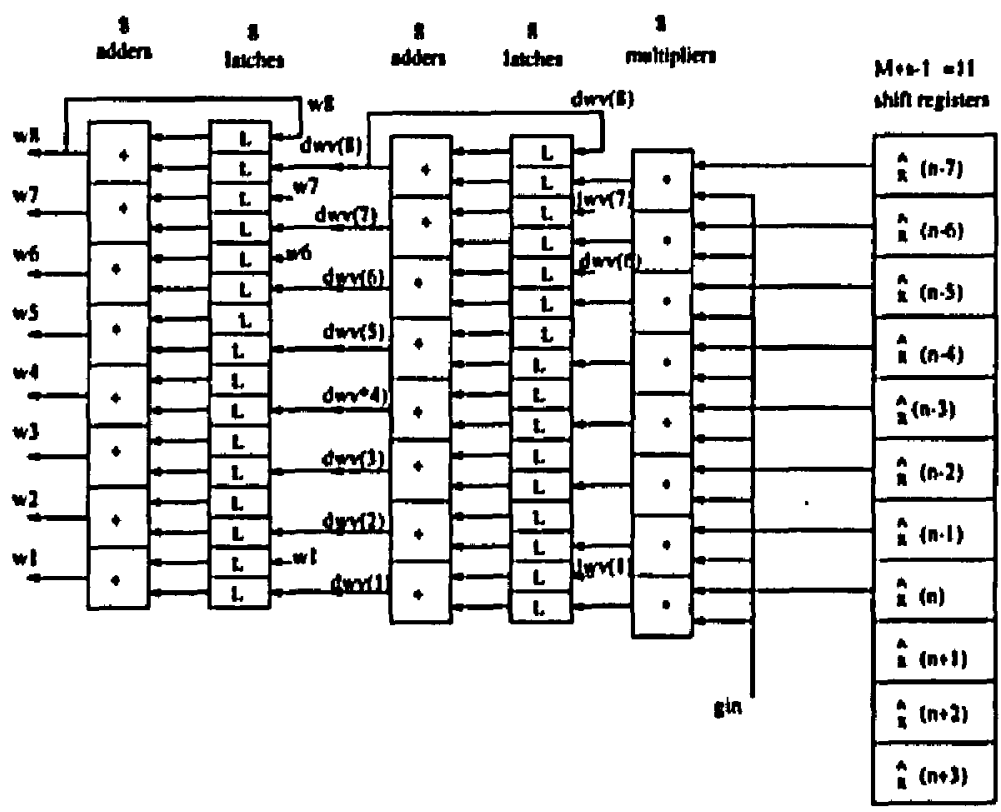

Figure 45: The configuration of the $\triangle$ block of PBS.8 architecture for an adaptive filter of order 8 and parallel step size 4 . 


\section{CHAPTER VI}

\section{Comparison of Binary Structured LMS algorithm, parallel LMS, and Fast Exact LMS}

The sequential LMS algorithm, as stated in equations $2.1,2.4$, and 4.3, has a complexity of $2 M$ multiplications and $2 M$ additions. Using single arithmetic unit, multiplier and adder, the time delay per iteration for LMS algorithm to update the tap weight vector is

$$
T_{L M I S}=2 M T_{\text {mult }}+2 M T_{a d d}
$$

This method is used in the MOTOROLA 56200 which uses a general purpose signal processor to perform the calculation part of an adaptive filter. In the recent years, with the ability to put many processors on a single chip, many parallel structures for LMS algorithm have been proposed. In the next two sections, two of these structures are described and their performance is evaluated. In the last section of this chapter, a comparison among these parallel algorithms and the Parallel Binary Structured LMS is performed.

\subsection{Parallel LMS ( P.LMS)}

The author in [25] present a simple parallel architecture for the LMS algorithm, called it Parallel LMS or P_LMS. The author proposes to use $M$ multipliers/accumulators plus $M$ adders to construct a parallel structure for an LMS based adaptive filter. $M$ is the order of 
the filter. The proposed architecture updates the tap weights after two multiplication and $\left\lceil\log _{2} M\right\rceil$ addition times.

The author suggests the structure for a filter of order 16. The time, area, and time_area performance of P.LMS with respect to the order of the filter is shown in Fig. 46(a), (b), and (c), respectively.

\subsection{Fast Exact LMS (FELMS)}

Authors in [24] present an algorithm, called Fast Exact LMS or FELMS, which obtains the results of the LMS algorithm in a parallel manner. The total number of multiplications and additions per iteration in their proposed algorithm is less than the total number of multiplications and additions requires in the sequential LMS algorithm.

In the FELMS algorithm, the scalar values of $s_{i}$ 's are calculated, recursively. Each $s_{i}$ is an inner product of two input vectors. $s_{i}$ 's are the elements of $N-1$ lower triangular $N \times N$ matrices, called $G_{i}$ 's. The product of these $N-1$ matrices is another $N \times N$ lower triangular matrix which is called $G(n)$. The elements of the error vector, $\varrho(n)$, is the result of multiplying $G(n)$ matrix to a vector. This vector is the differenee between desired output vector and the actual output vector. The tap weight vector, $H(n+1)$, is modified using the error vector and the values of the new inputs to the filter.

To implement different parts of the FELMS algorithm, different architectures are examined. The area and time estimation for each part of the FELMS algorithm is described in Appendix $A$.

Consider two methods of implementing the FELMS algorithm. The first one is the fastest method, from time delay point of view, and the other is the smallest method, from 


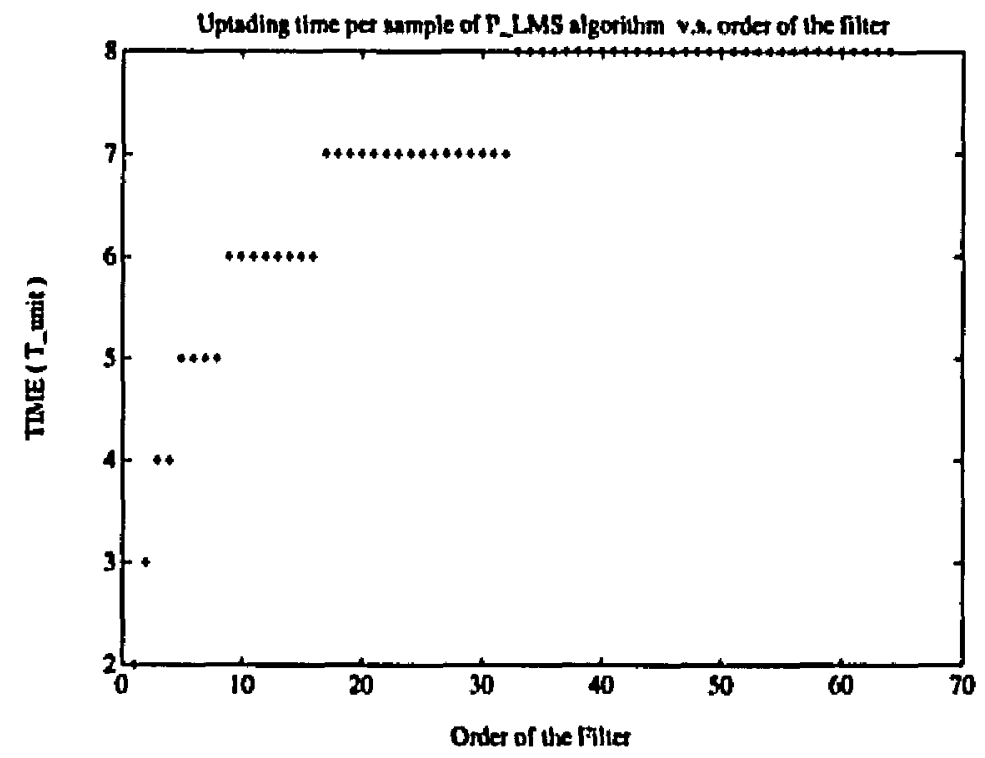

(a)

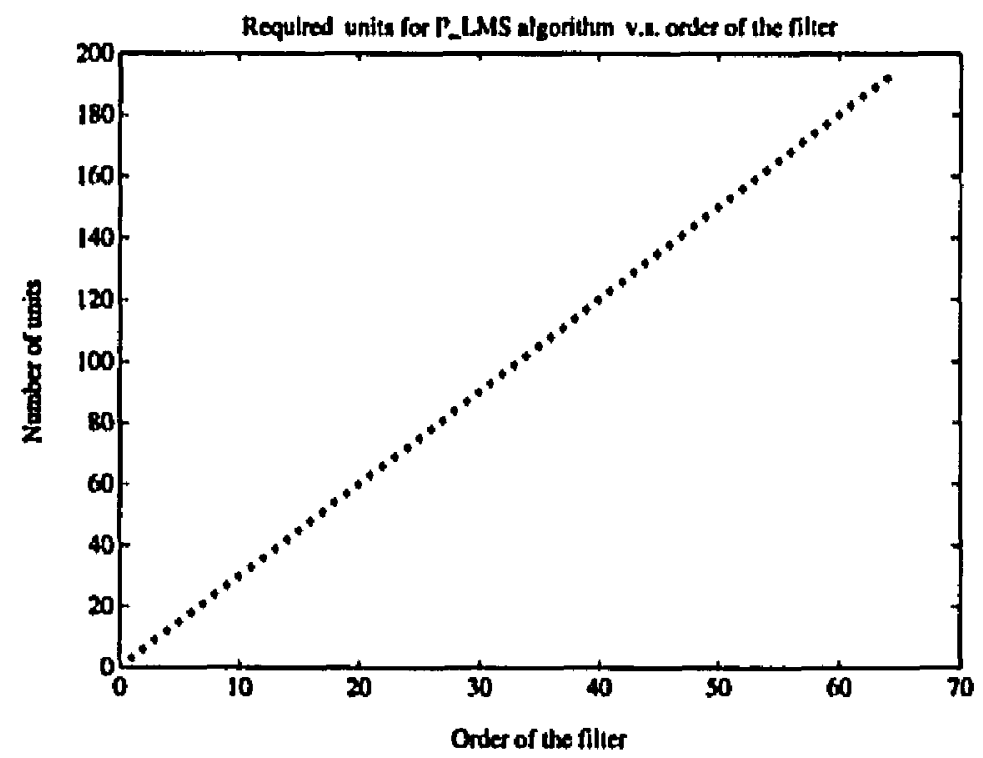

(b)

Figure 46: Time, area and time_area performance of P LMS algorithm versus order of the filter; (a) Total time delay per sample for PLMS algorithm; (b) Total area required for P.LMS algorithm; (c) Time_area performance of P_LMS algorithm. 
Fig. 46(continued)

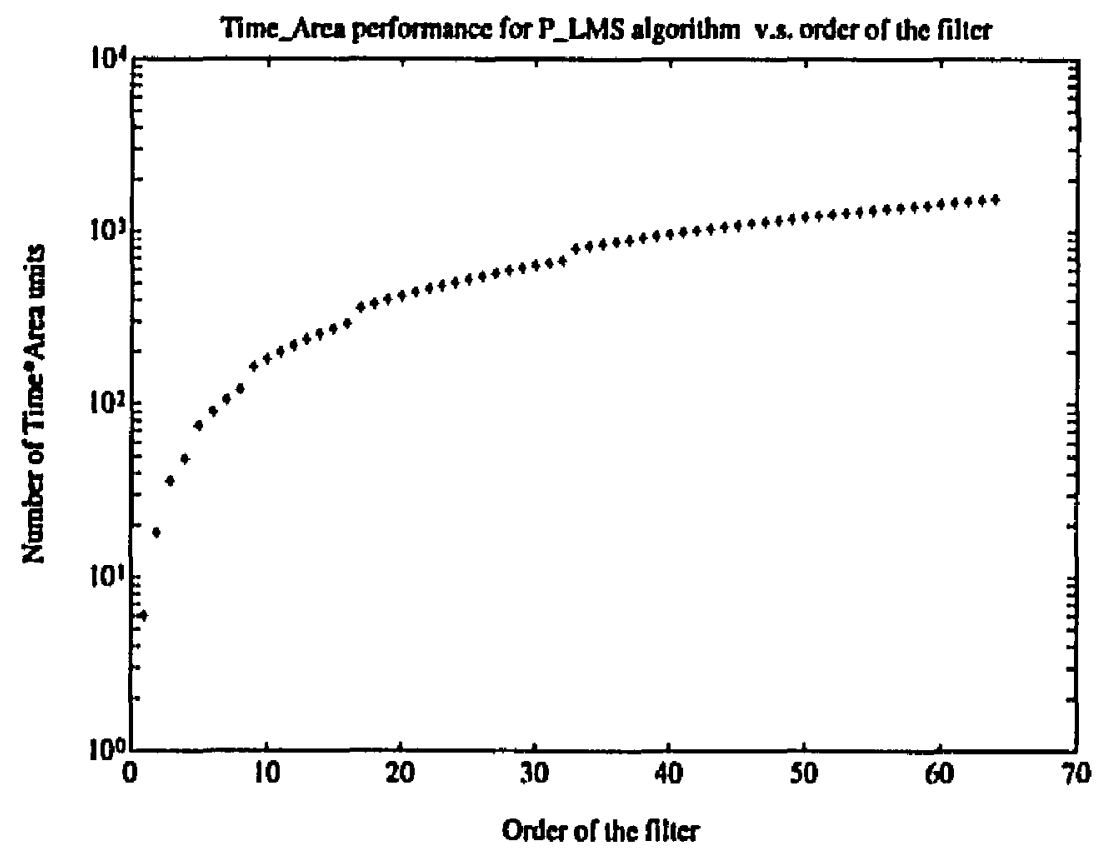

Fig. 46(c)

the number of units and area point of view. The fastest method, call it FELMS_I, has total time delay of

$$
\begin{aligned}
T_{\text {total-feldis-1 }}= & (N+2) T_{\text {mult }}+ \\
& \left(Z+2 N+\left[\log _{2}(L)\right\rceil+4\left\lceil\log _{2}(N)\right\rceil+1\right) T_{\text {add }}
\end{aligned}
$$

where

$$
Z=\sum_{k=1}^{N-2}\left[\log _{2}(k)\right]
$$


The total number of unit of FELMS_l is

$$
\begin{aligned}
A_{\text {total-FELMS }-1}= & \left(Z+\frac{N^{3}}{2}+\frac{3 N^{2}}{2}-\frac{3 N}{2}+(N-1) L\right) A_{m u l l}+ \\
& \left(Z+\frac{5 N^{2}}{2}+\frac{5 N}{2}-\frac{2 L}{N}+N L+5 L\right) A_{\text {add }}
\end{aligned}
$$

The smallest circuit for FELMS, FELMS_2, has time delay of

$$
\begin{aligned}
T_{\text {total-FELMS } 2=} & \left(\frac{9 N^{2}}{2}-3 N+1\right) T_{\text {mult }}+ \\
& \left(4 N^{2}+2 N+2 N\left\lceil\log _{2}(N)\right]+\left[\log _{2}(L)\right]+\left[\log _{2}(N)\right\rceil-3\right) T_{\text {add }}
\end{aligned}
$$

with total number of unit of

$$
\begin{aligned}
A_{\text {total-FELMSS2 }}= & \left(2 N^{2}+N L+N-L-1\right) A_{\text {mult }}+ \\
& \left(3 N^{2}+3 N-\frac{L}{N}+N L+3 L-1\right) A_{\text {add }}
\end{aligned}
$$

The average time delay per iteration for each FELMS is

$$
T_{\text {FELMS }}=\frac{T_{\text {total-FELMS }}}{s}
$$

With the same assumption made in the previous chapter, Eq. 5.10, 5.11, and 5.12, Fig. 47 and 48 depict the time, area, and time_area performance of two FELMS methods with respect to the step size, $s$, and order of the filter, $M$, respectively. FELMS_I has the best performance in the time and time_area evaluations, Fig. 47(a), 47(c), 48(a), and 48(c). Therefore FELMS_ 1 is chosen to represent FELMS algorithm in the next section where the performances of the three parallel algorithms for LMS are compared. 


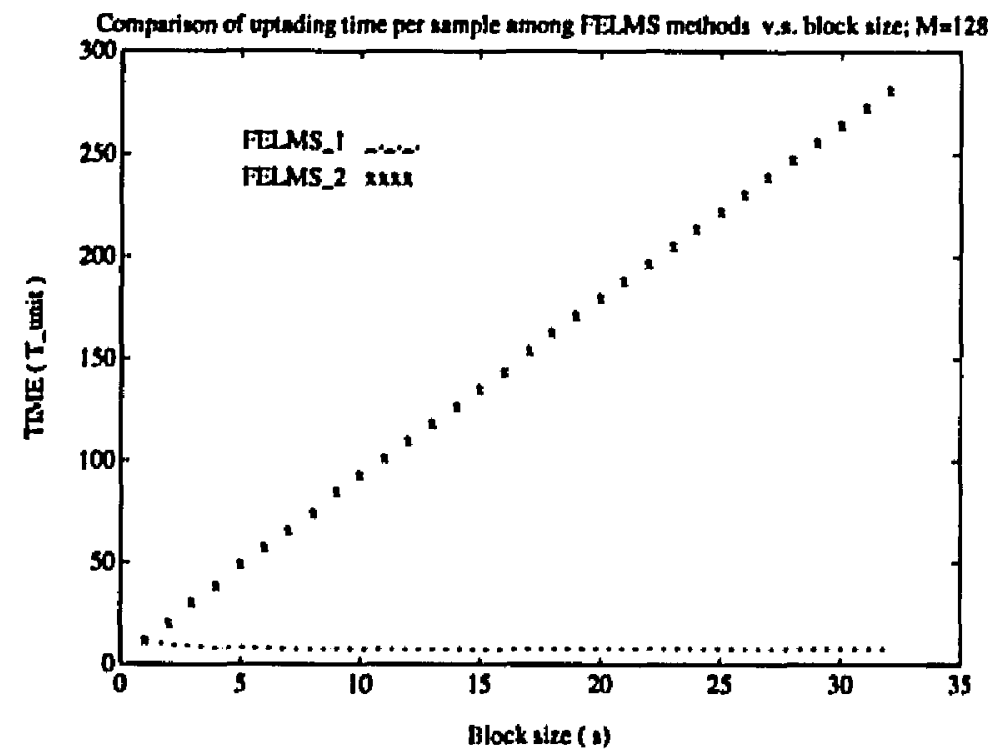

(a)

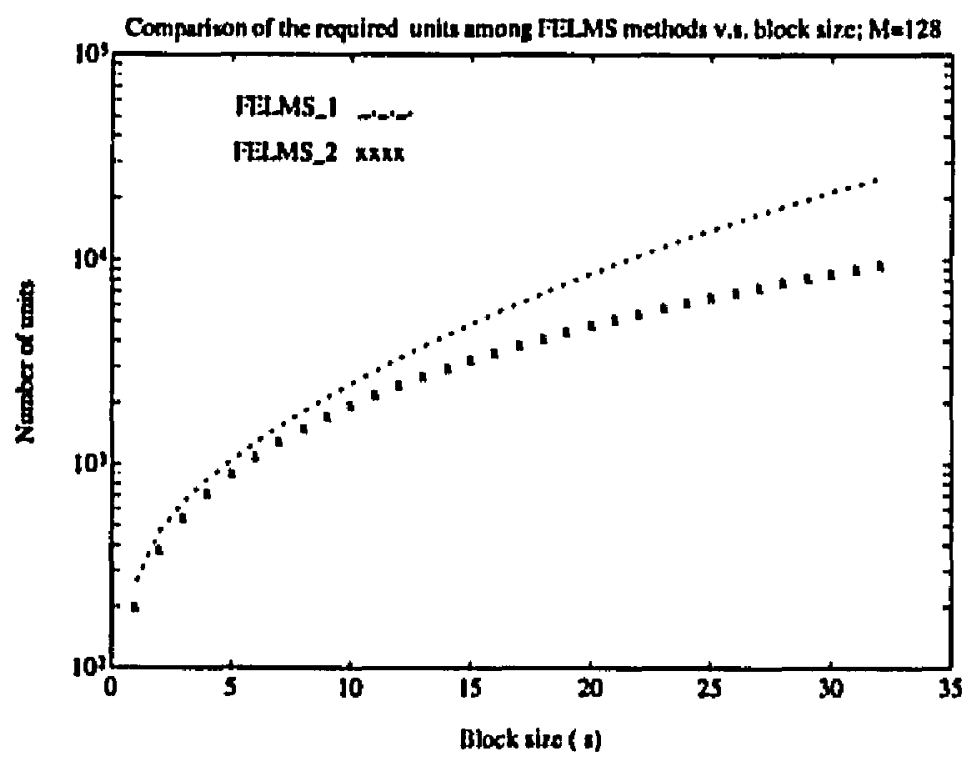

(b)

Figure 47: Comparison between the different methods of implementing the FELMS algorithm versus block size $s$. (a) Total time delay per sample for FELMS algorithm. (b) Total area required for FELMS algorithm. (c) Time area presentation of different FELMS methods 
Fig. 47(continued)

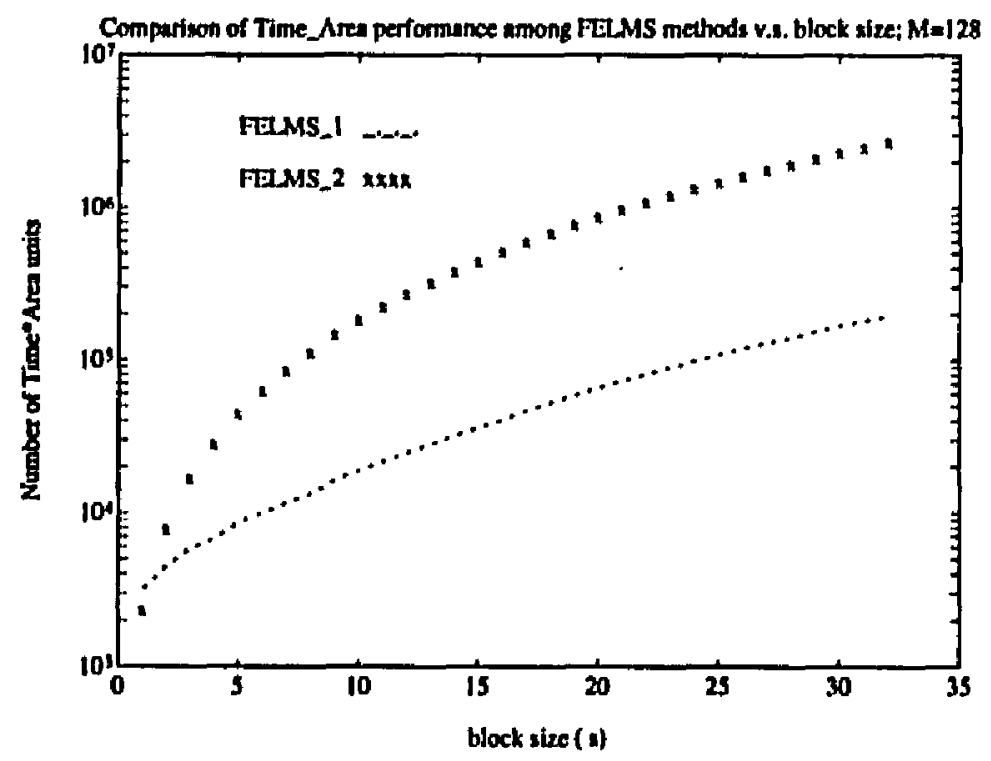

(c)

\subsection{Comparison of the performance of PBSLMS architectures with PLMS, and FELMS}

Eight possible architectures of PBS.LMS algorithm are evaluated in the previous chapters. Figure 49 shows a comparison between those eight PBS_k's and P_LMS for different step size $s$, when order of the filter is constant, $M=128$. Figure 49(a) shows the delay times of the architectures, Fig. 49(b) shows a comparison between the total number of units required in each architecture, and Fig. 49(c) is the time.area criterion of the architeclures. The effect of the order of the filter on the three performance criteria, time, area, and time_area, between PBS's and PLMS is depicted in 50(a), (b), and (c), respectively. 


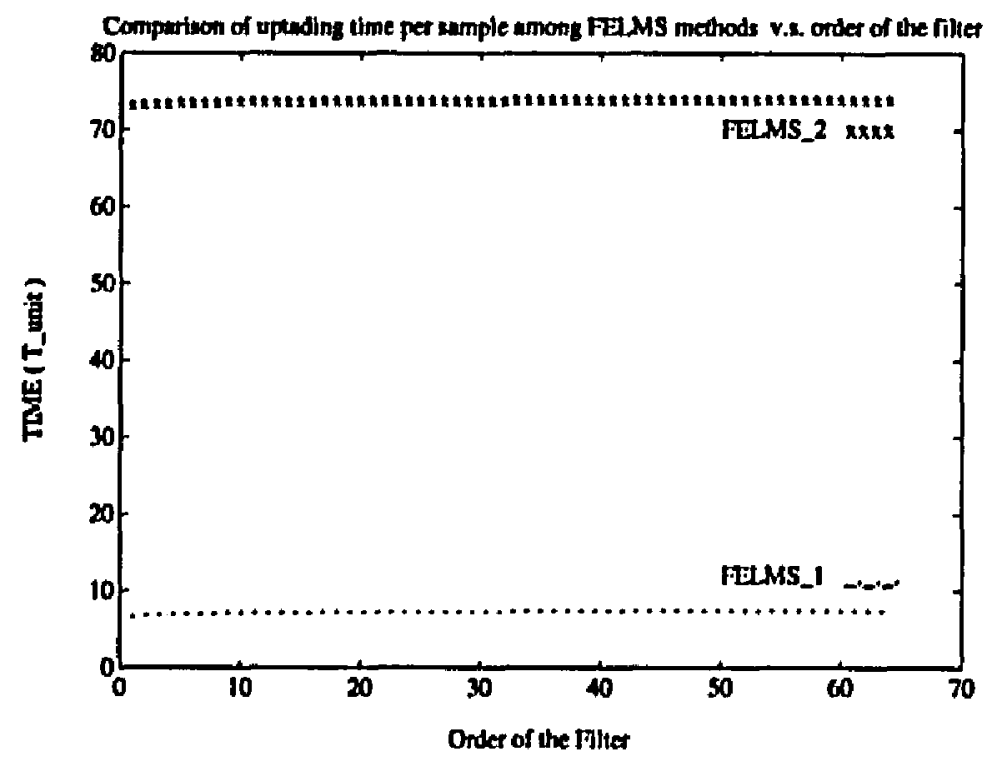

(a)

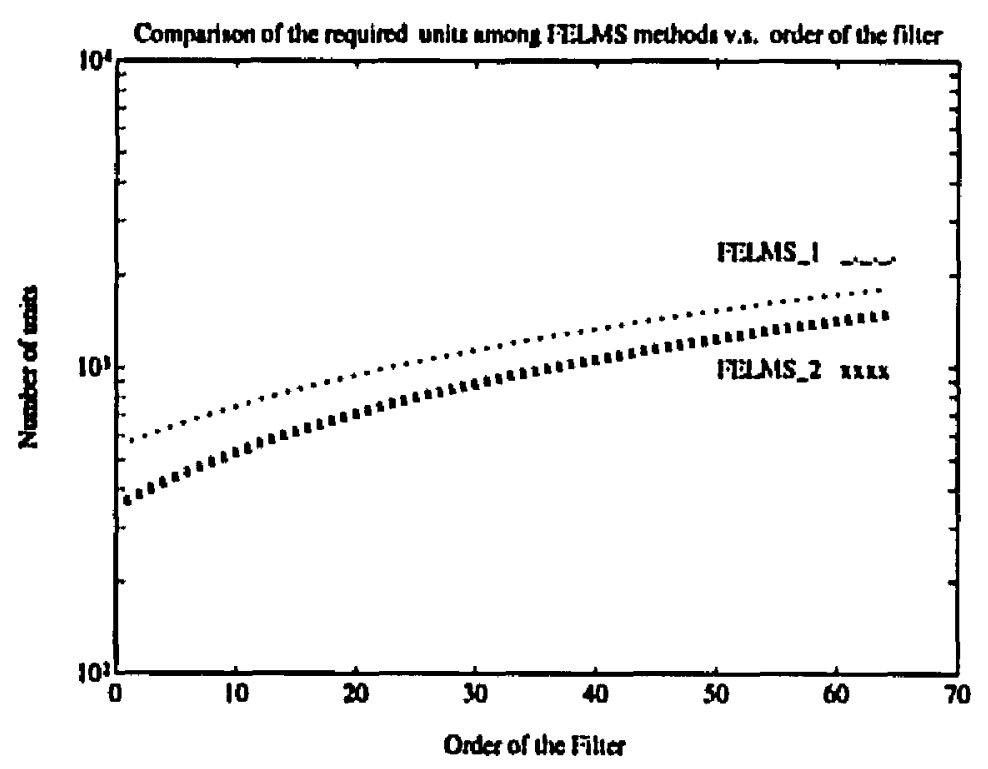

(b)

Figure 48: Comparison between the different methods of implementing the FELMS algorithm versus order $M$. (a) Total time delay per sample for FELMS algorithm. (b) Total area required for FELMS algorithm. (c) Time_area presentation of different FELMS methods 
Fig. 48(continued)

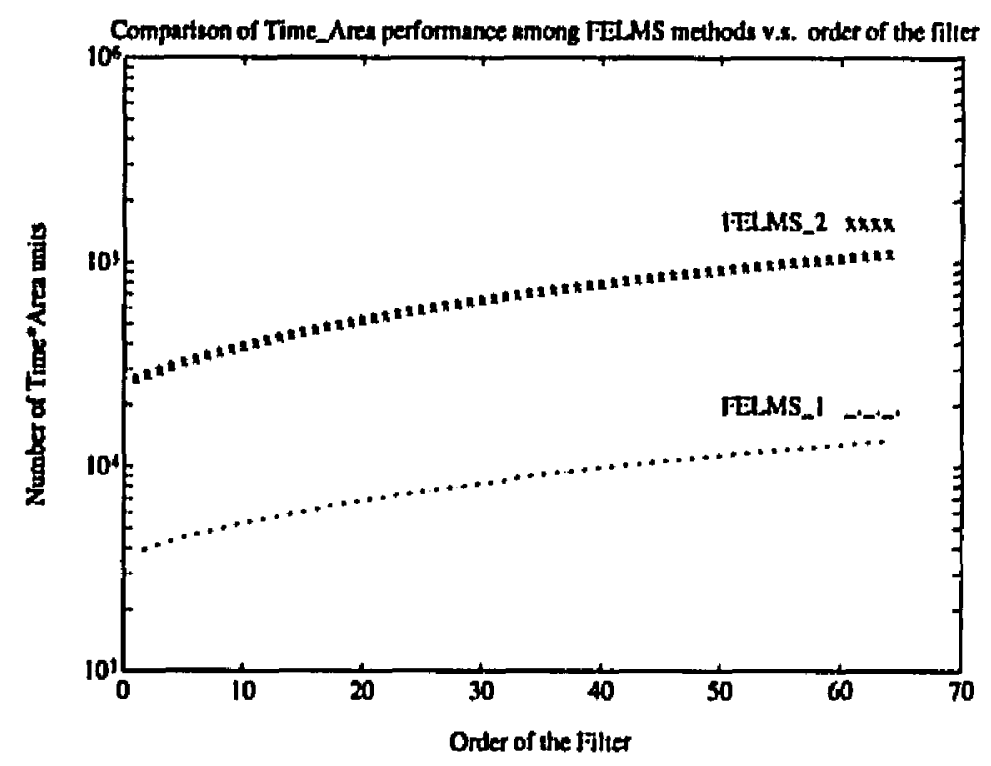

(c)

The same comparisons of time, area, and time_area performance between PBS_LMS architectures and the best architecture of FELMS, FELMS_1, is depicted in Fig. 51 and 52. Figure 51 shows these comparison with respect to the step size s. Fig. 52 shows the effect of the order of the filter on the performances of the proposed parallel architectures.

As it is clear from these figures, two of PBS.LMS architectures are the best among the others. PBS 2 is not the fastest method, since PBS.1 is the fastest approach for the adaptation process. However in the area and time_area performance, the best choice between PBS_1 and PBS_2 is PBS_2. In the other hand PBS_8 is the smallest structure for PBS_LMS algorithms. Even though in the time delay performance PBS_8 is the slowest 


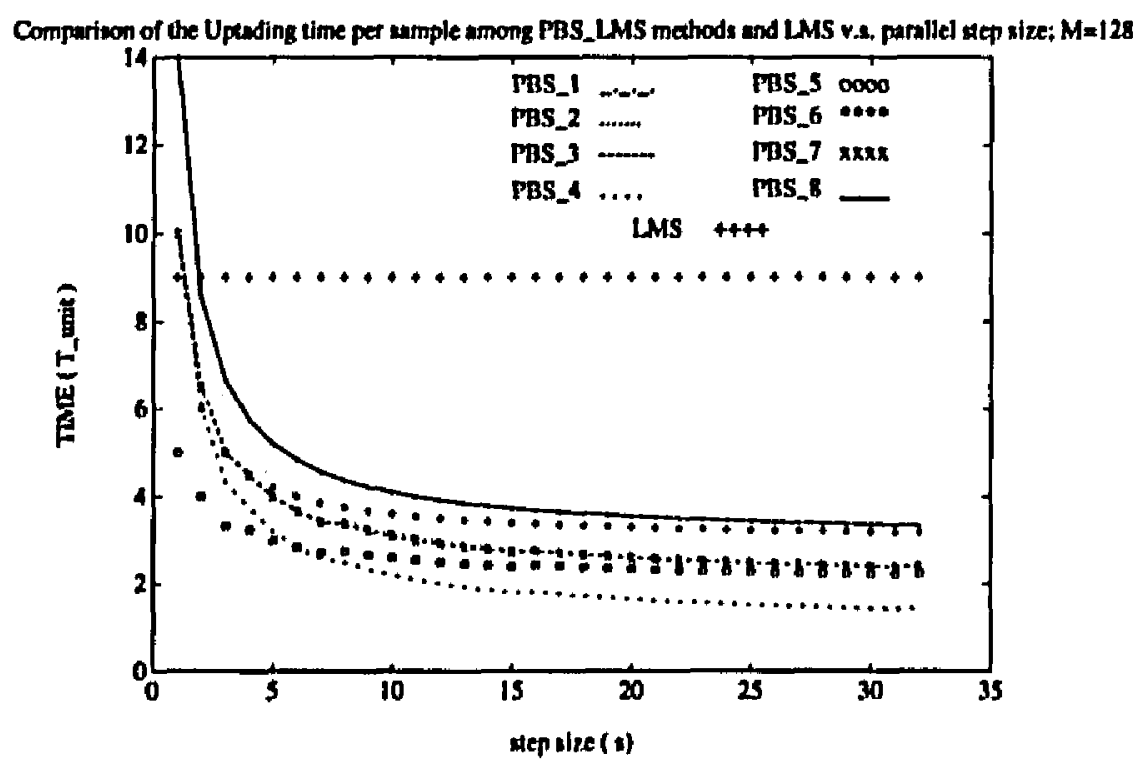

(a)

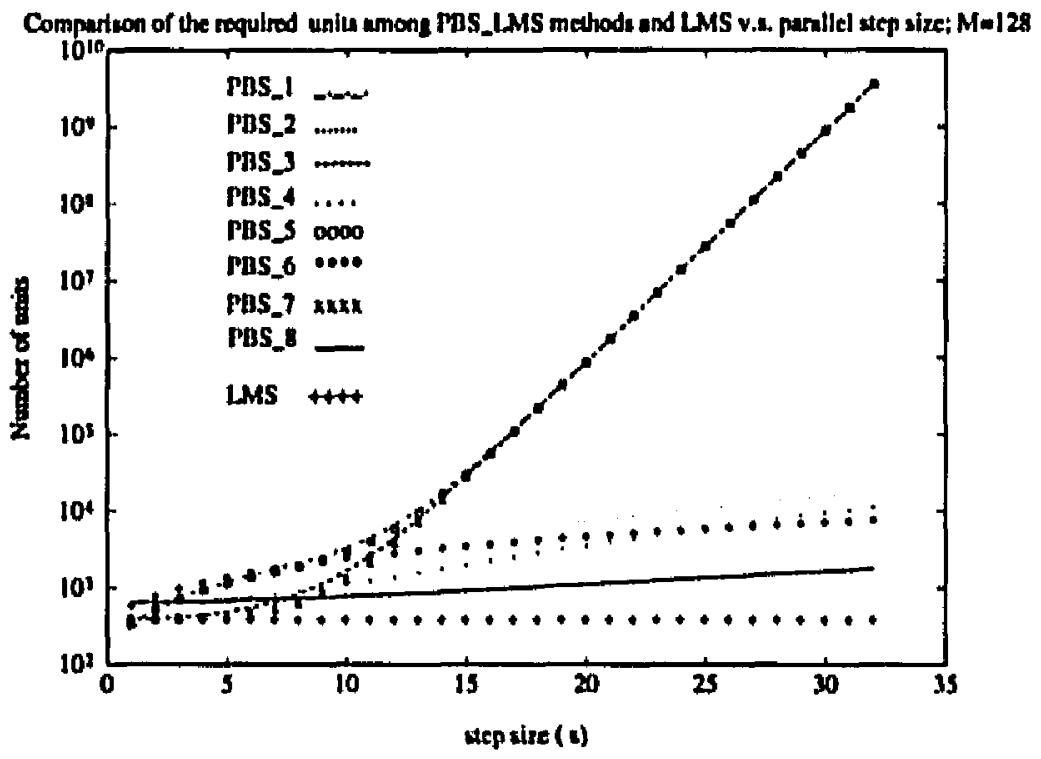

(b)

Figure 49: Comparison between the different methods of implementing the PBS.LMS and P.LMS versus step size $s$. (a) Total time delay per sample; (b) Total area required; (c) Time_area performance of different methods 
Fig. 49(continued)

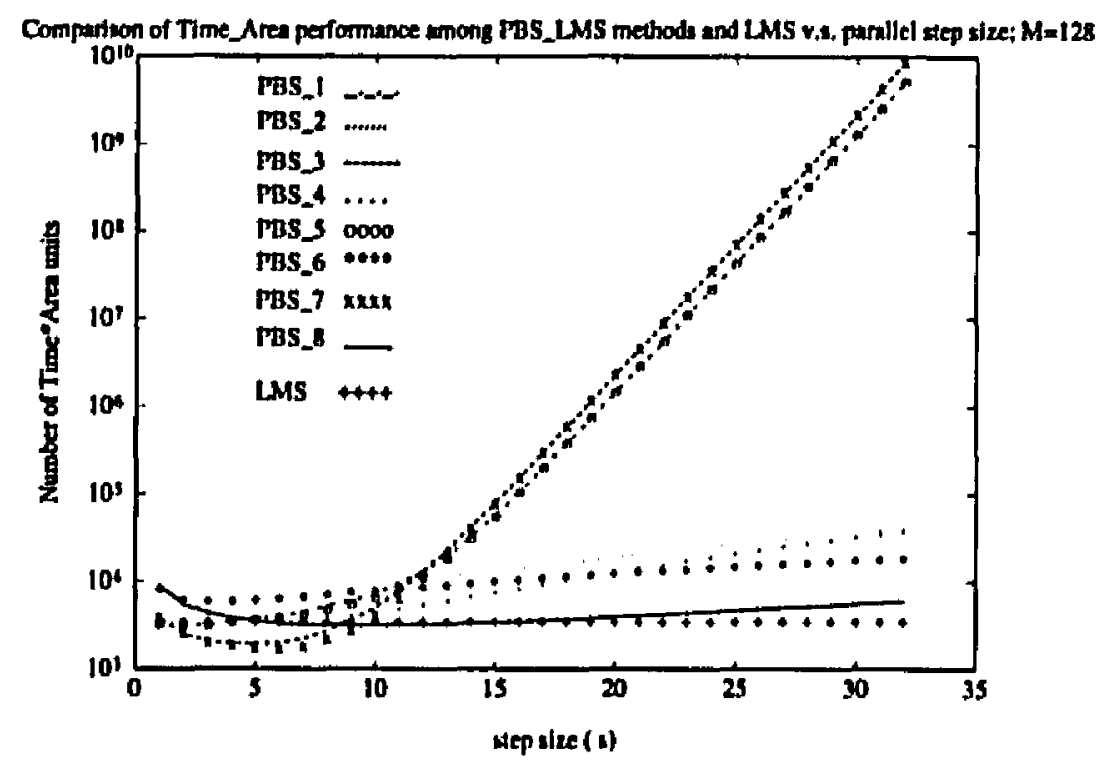

(c)

circuit. Thus, PBS 2 and PBS .8 , the optimum fastest and smallest structures of PBS_LMS, are chosen to be evaluated in the more details. In this part, the performance of PBS_2 and PBS_8 are compared with FELMS 1 and PLMS. In the next chapter the result of VHDL simulations of these two PBS LMS algorithms are presented.

Fig. 53 and Fig. 54 show the a comparison among the performance of PBS_2, PBS_8, FELMS.I, and P.LMS with respect to step size and order of the filter, respectively. The speed up two PBS 2 and PBS_8 and FELMS_I with respect to P_LMS are shown in Fig. 55. Fig. 55(a) shows the speed ups versus step size, when the order of the filter is constant, $M=128$. Fig. 55(b) shows the speed ups versus order of the filter for a constant $s=8$. 


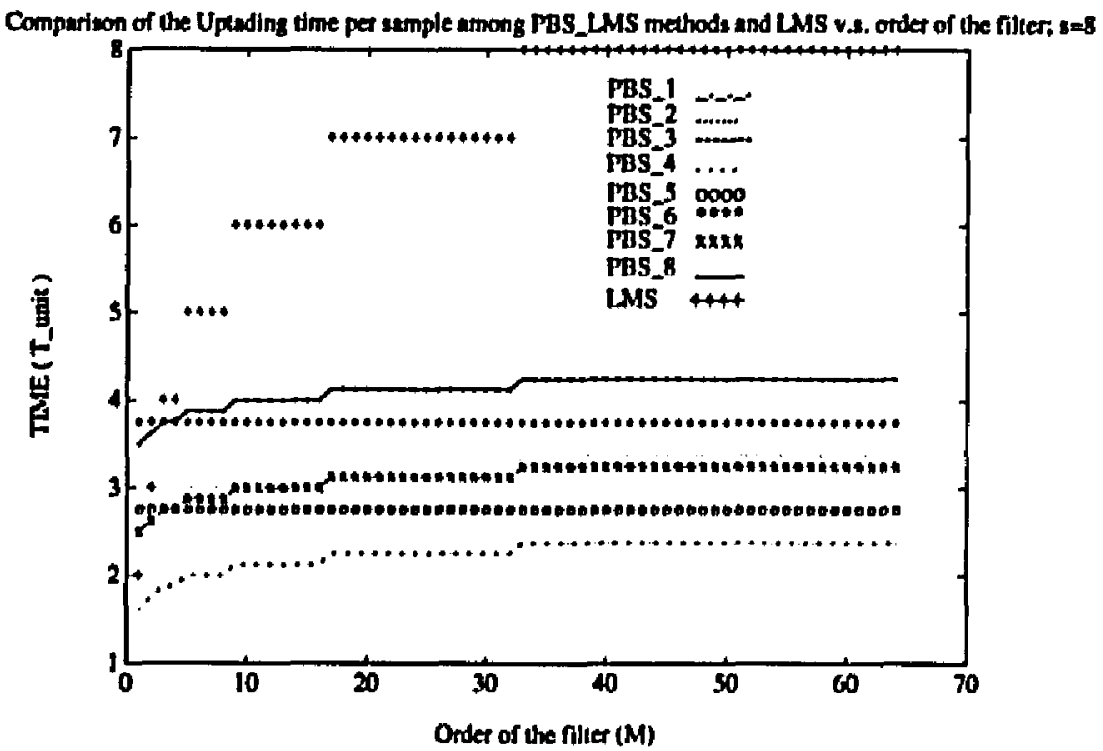

(a)

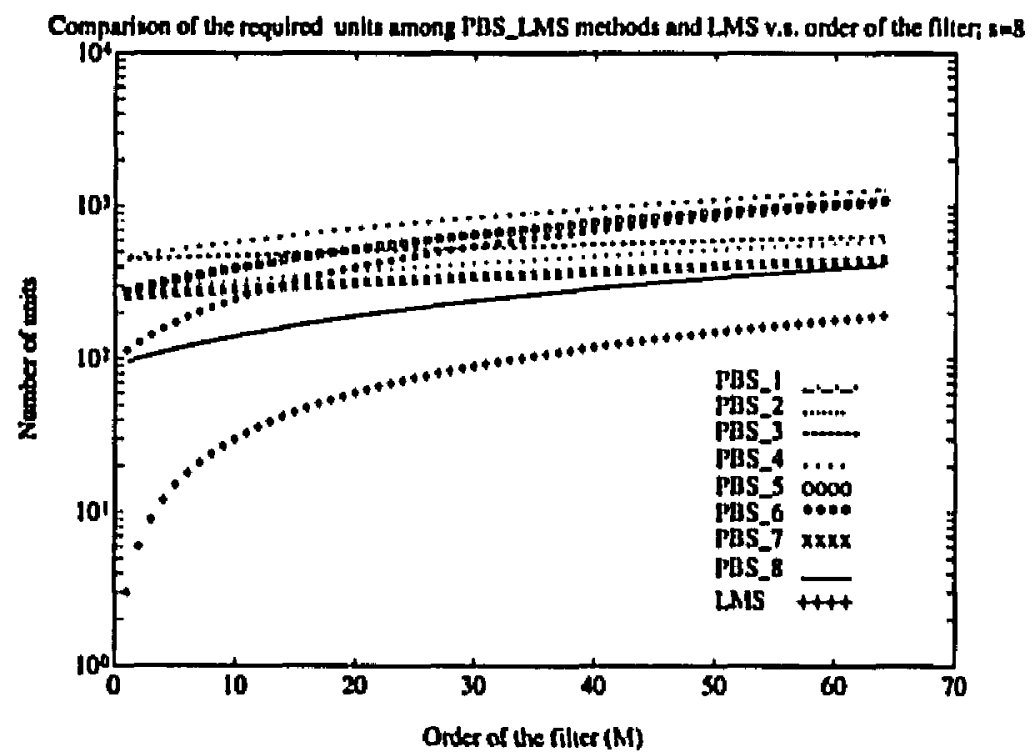

(b)

Figure 50: Comparison between the different methods of implementing the PBS.LMS and P.LMS versus order of the filter. (a) Total time delay per sample; (b) Total area required; (c) Time_area performance of different methods 
Fig. 50(continued)

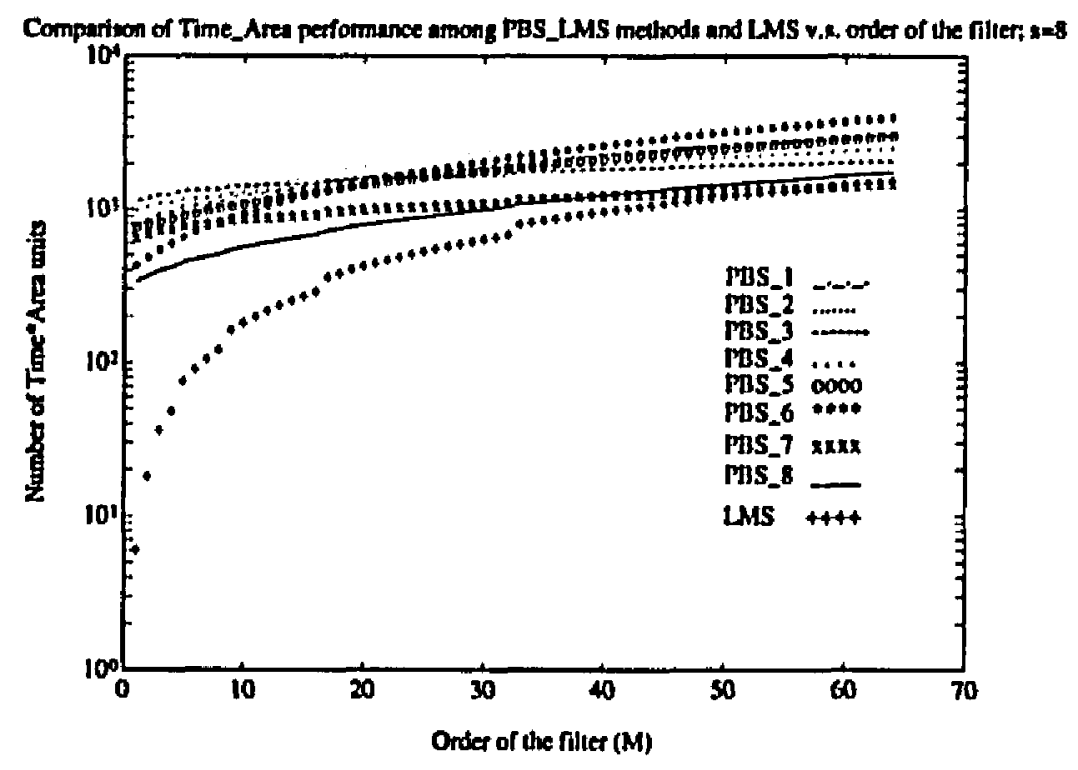

(c)

These figures show that PBS_8 architecture has a time_are performance compatible to the P_LMS architecture. Even for some values of the step size, $6<s<14$, the LMS_8 has smaller time_area product than the P.LMS. In the same range for the step size, the LMS_8 is two times faster than the P LMS.

The result of a computer simulation of two adaptive filters one based on PBS_8 and the other based on the P.LMS algorithms is shown in Fig. 56. In this simulation, the gradient step size and the eigenvalue spread of the random processes are constant, $\mu=0.005$, $\Lambda=50$. The simulation is run for $300 \mu s e c$ and the results were averaged over 50 trials. In this simulation, it is assumed that $T_{\text {mult }}=T_{\text {add }}=100$ nsec. Fig. 56 shows the average 


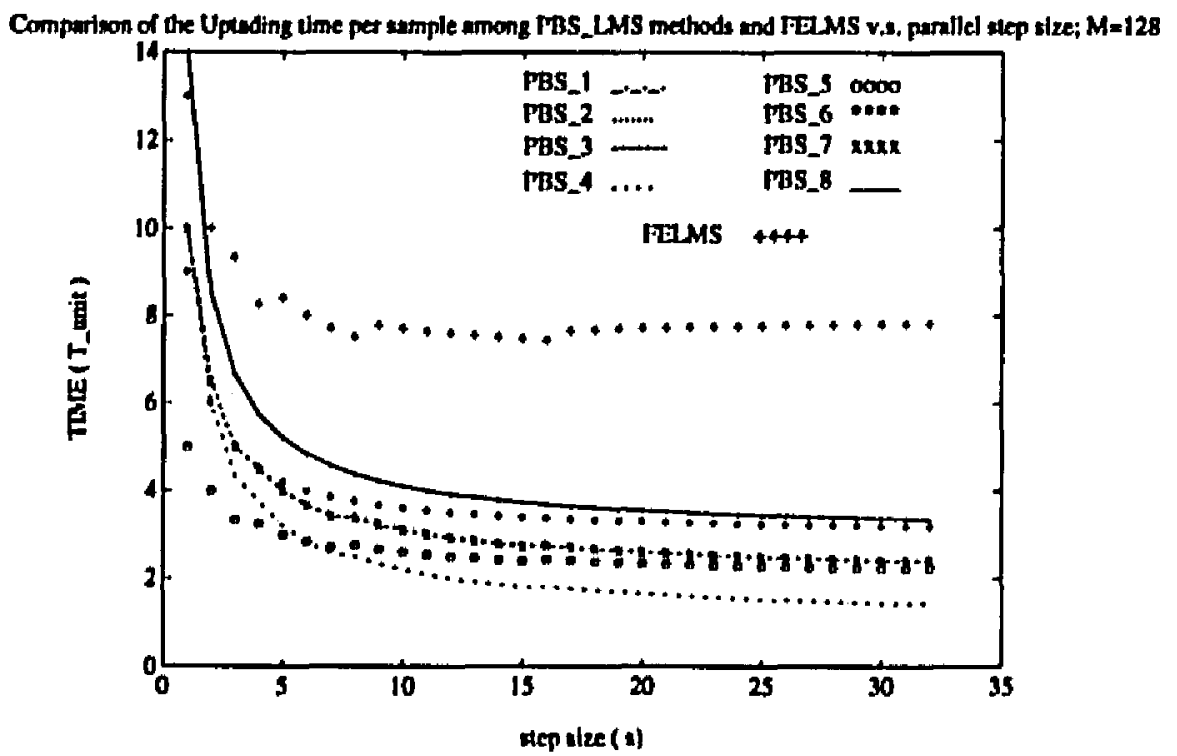

(a)

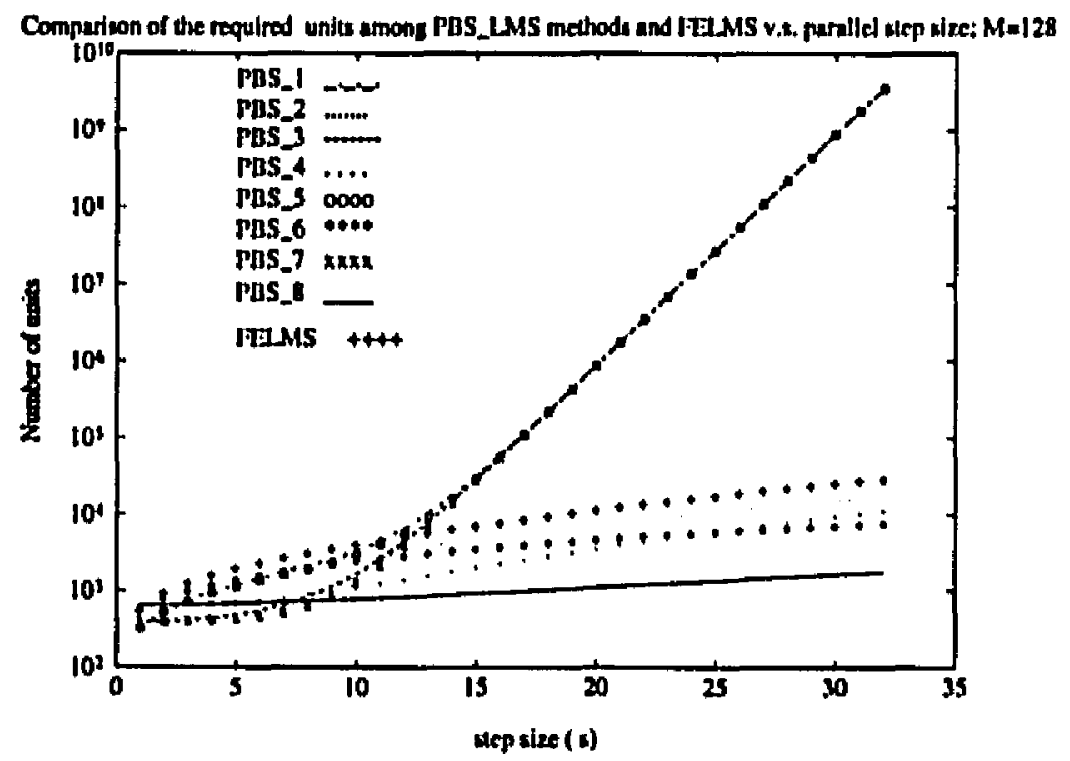

(b)

Figure 51: Comparison between the different methods of implementing the PBS LLMS and FELMS versus step size s. (a) Total time delay per sample; (b) Total area required; (c) Time_area performance of different methods 
Fig. 51(continued)

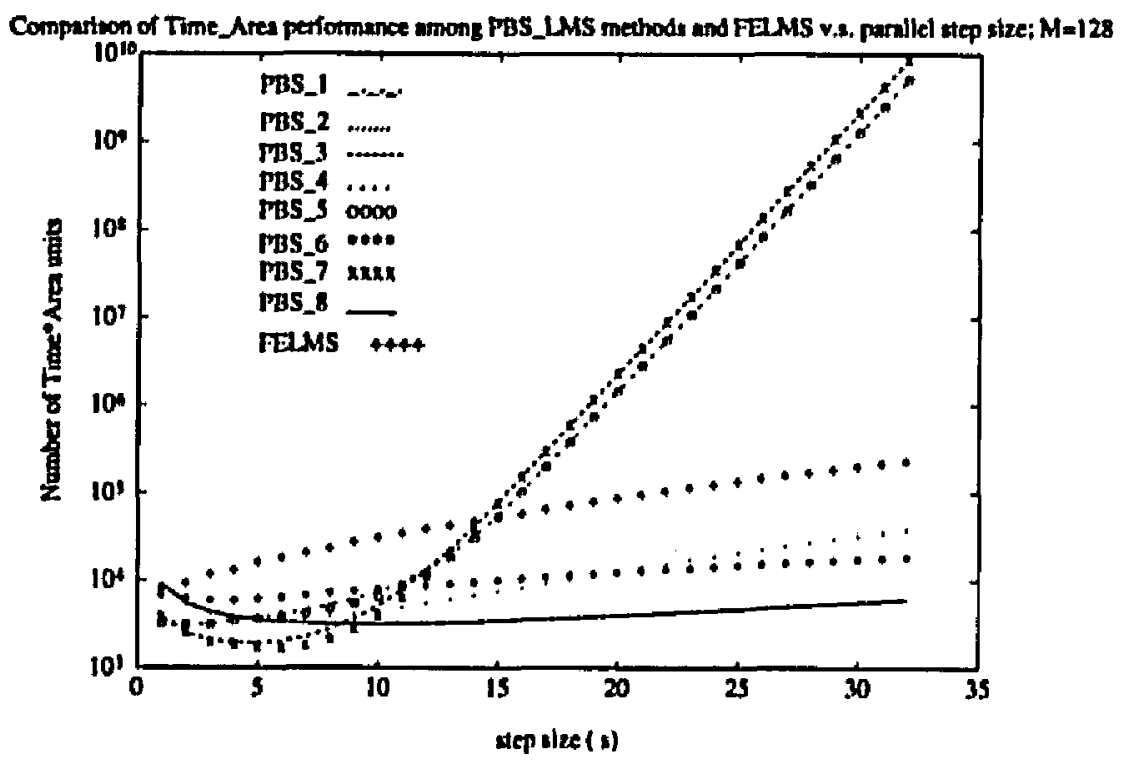

(c)

of the square of the errors of two adaptive filters versus time. This figure shows that the adaptive filter based on the PBS.8 Fig. 57 depiets the details of the same average of the square of the errors of two adaptive filters. 


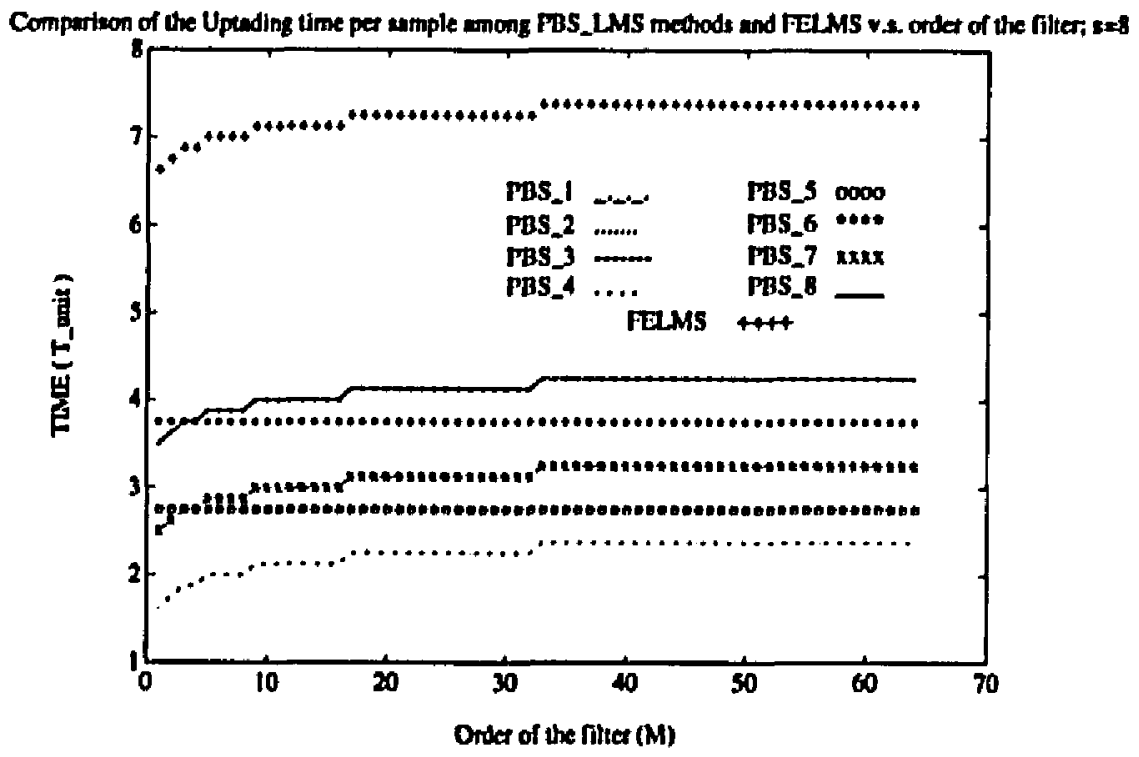

(a)

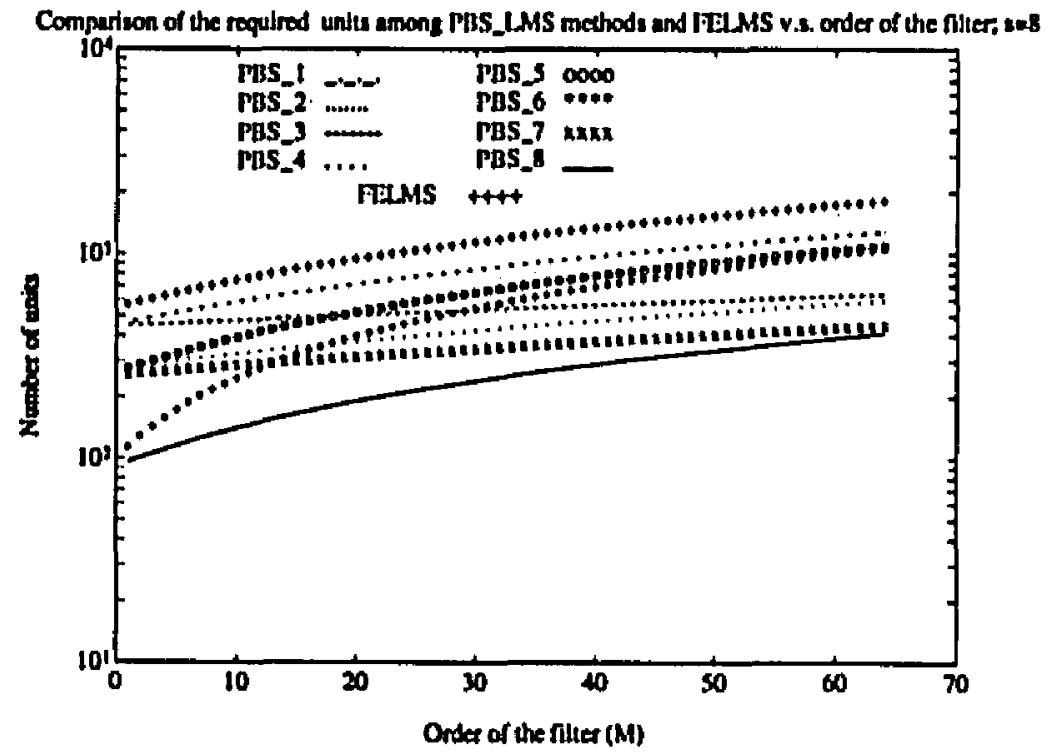

(b)

Figure 52: Comparison between the different methods of implementing the PBS_LMS and FELMS versus order of the filter; (a) Total time delay per sample; (b) Total area required; (c) Time area performance of different methods. 
Fig. 52(continued)

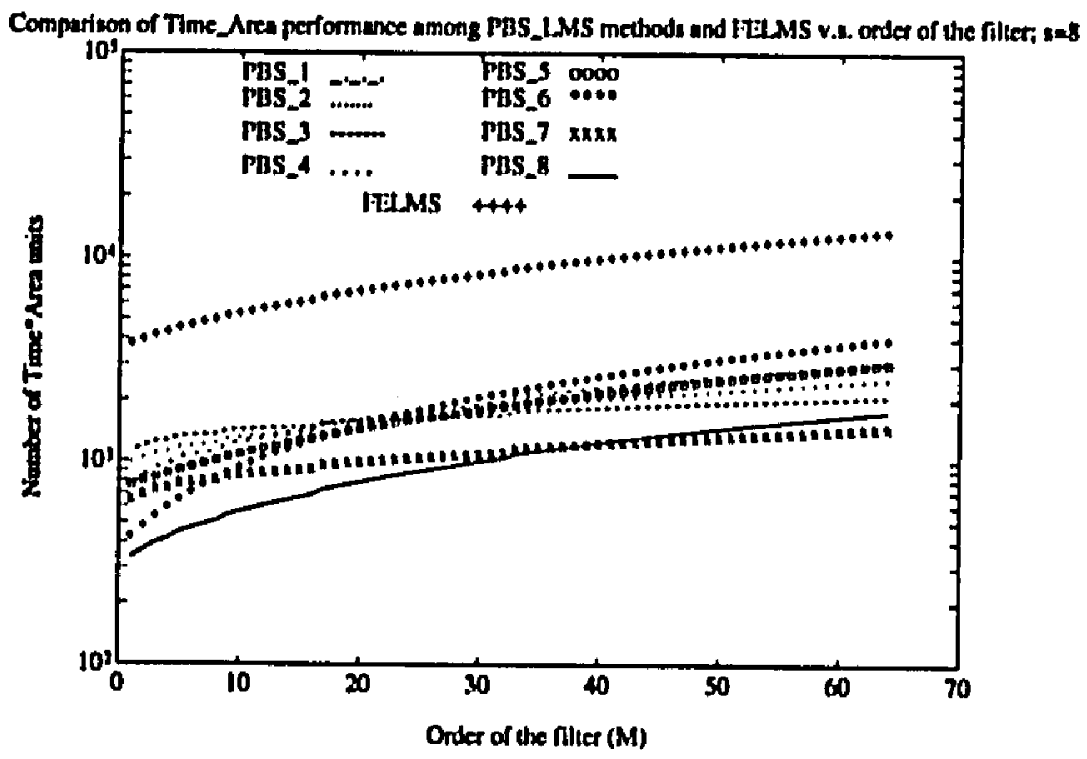

(c) 


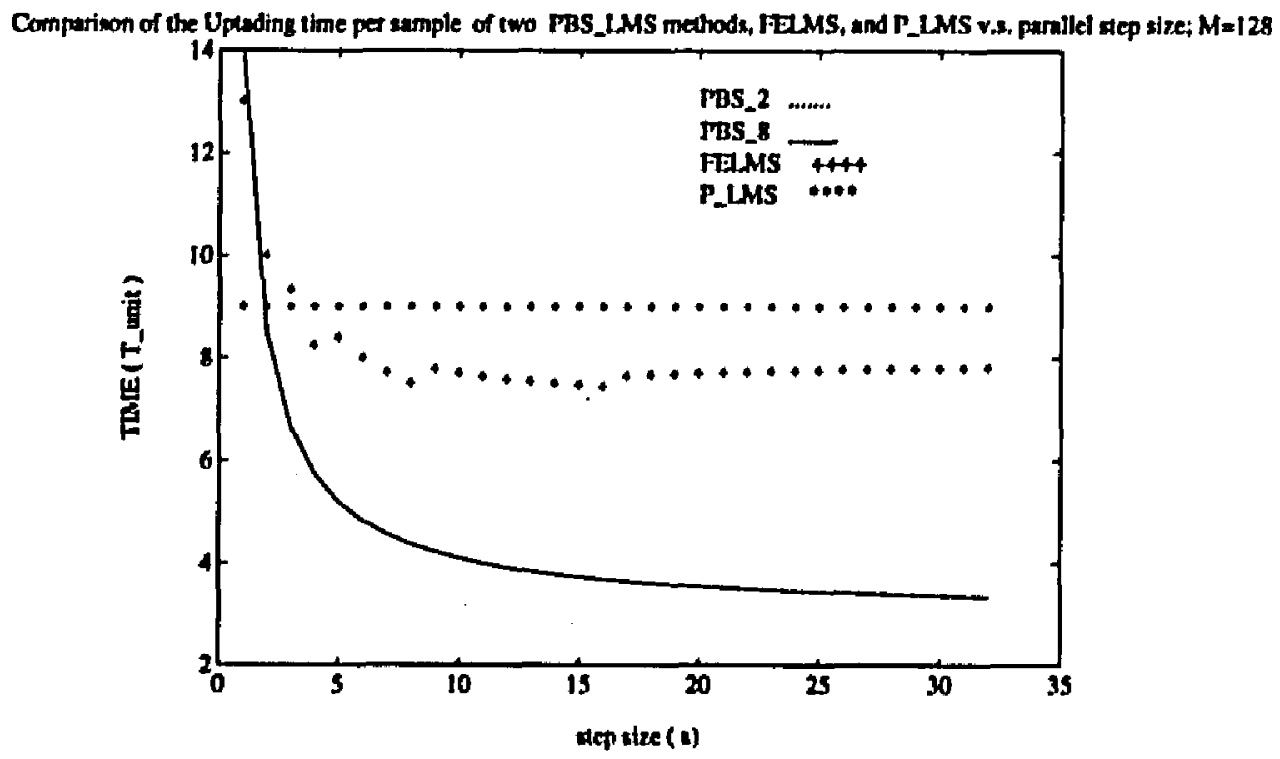

(a)

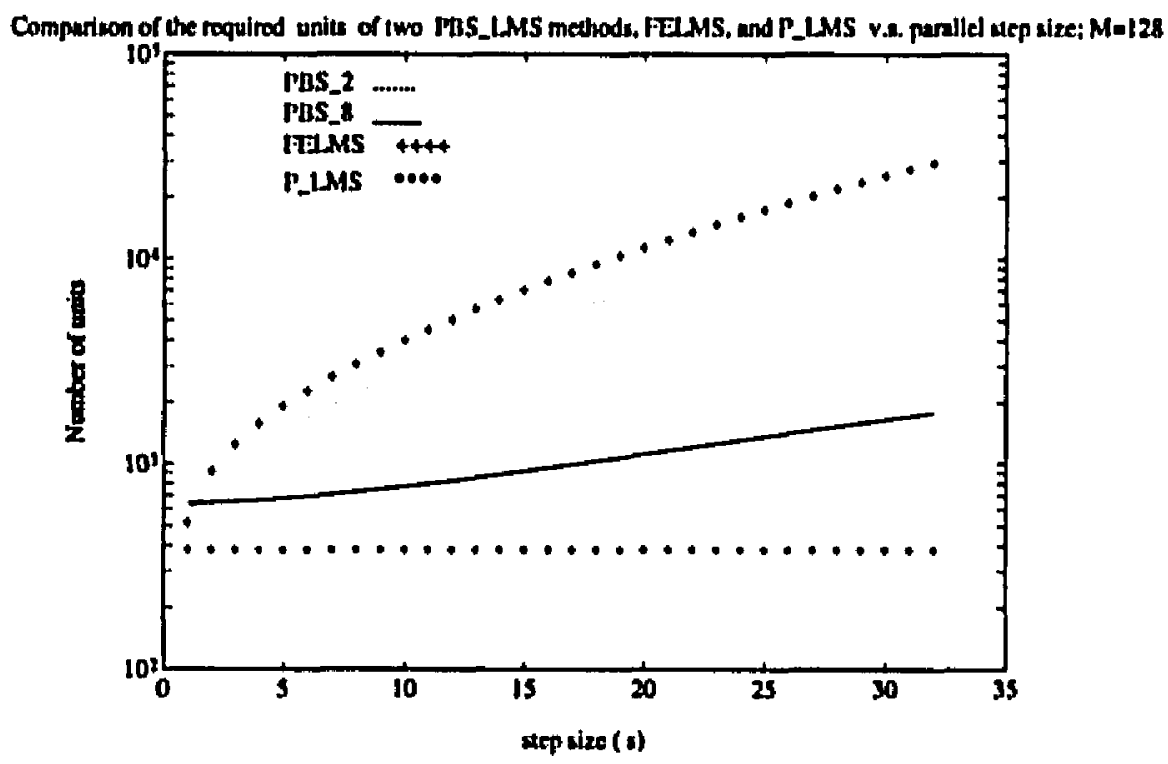

(b)

Figure 53: Comparison between the performance of the PBS_2, PBS_8, FELMS, and P.LMS versus step size $s$. (a) Total time delay per sample; (b) Total area required; (c) Time_area performance of different methods 
Fig. 53(continued)

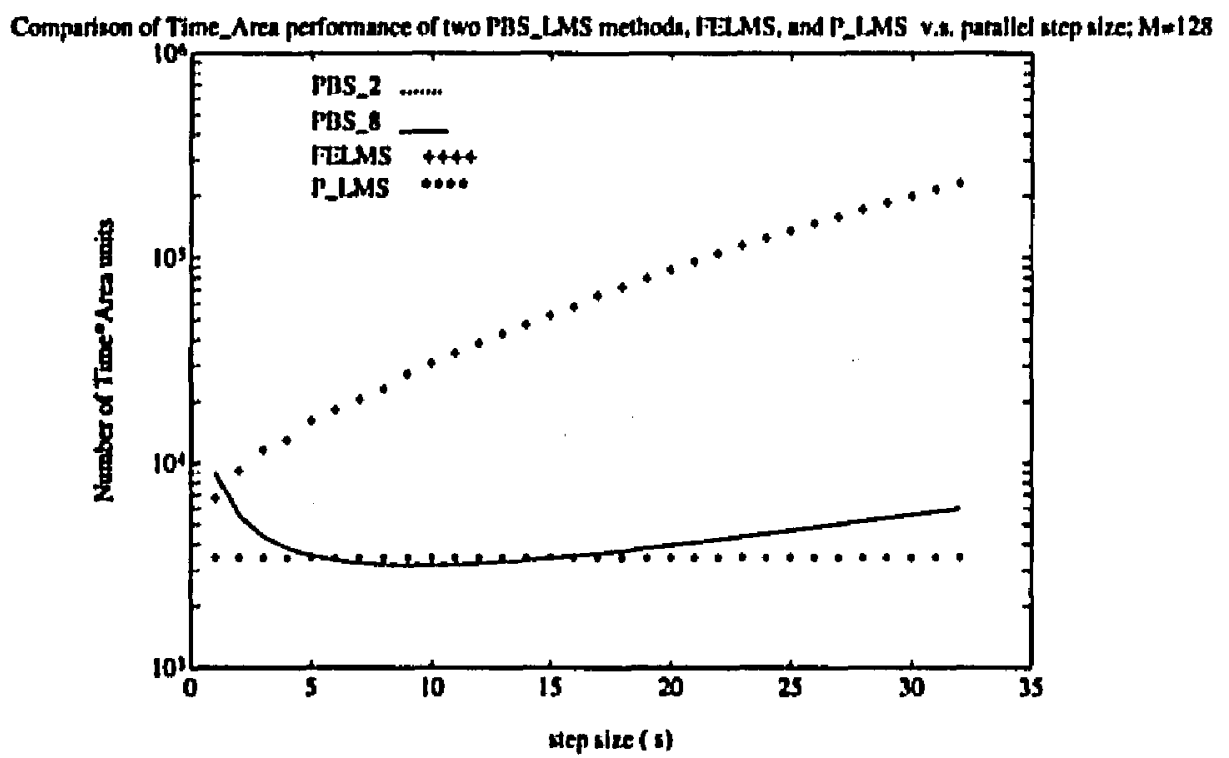

(c) 


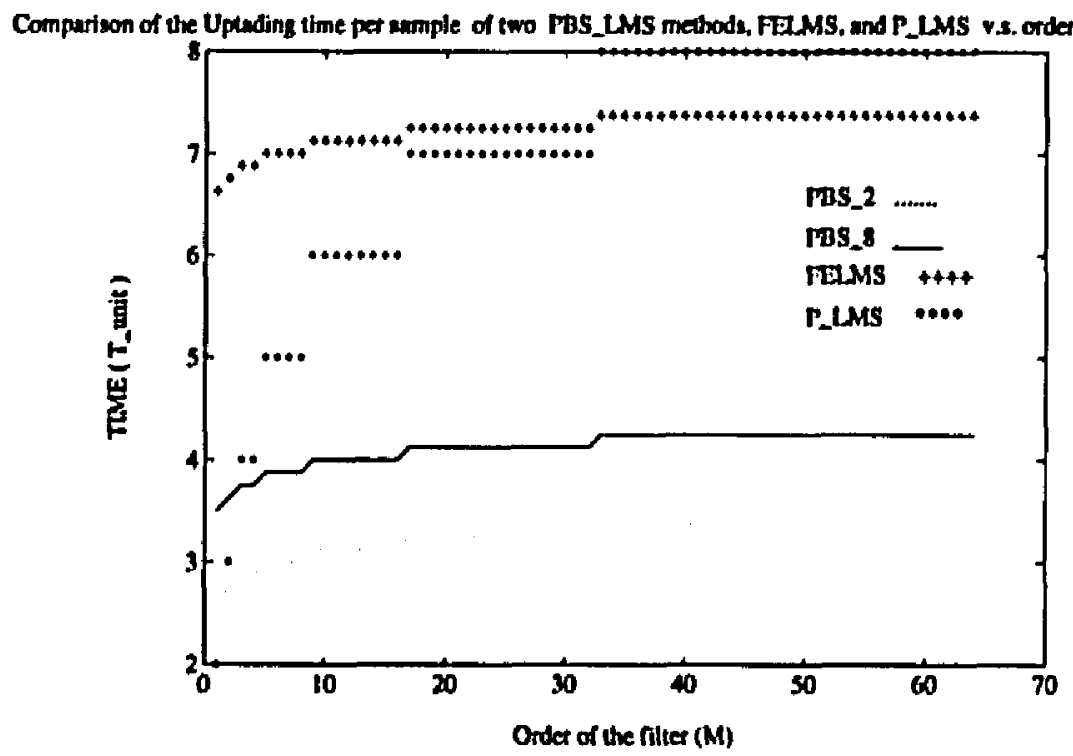

(a)

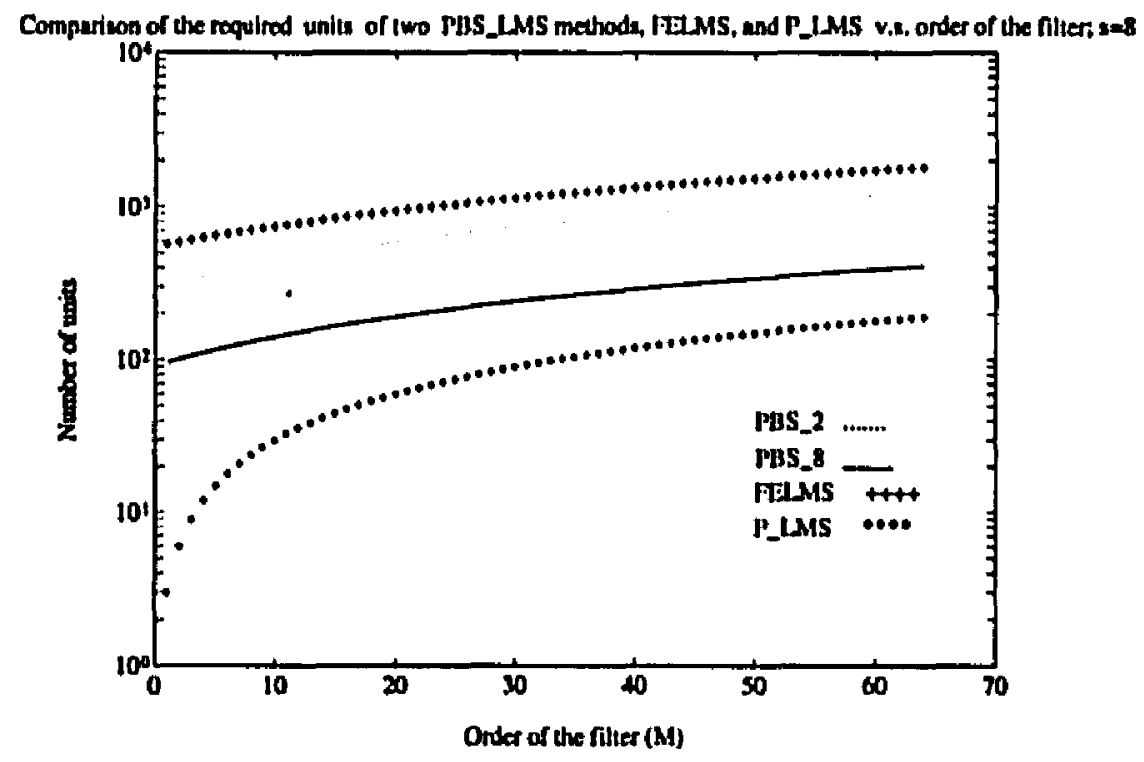

(b)

Figure 54: Comparison between the performance of the PBS_2, PBS_8, FELMS, and P LMS versus order of the filter. (a) Total time delay per sample; (b) Total area required; (c) Time_area performance of different methods 
Fig. 54(continued)

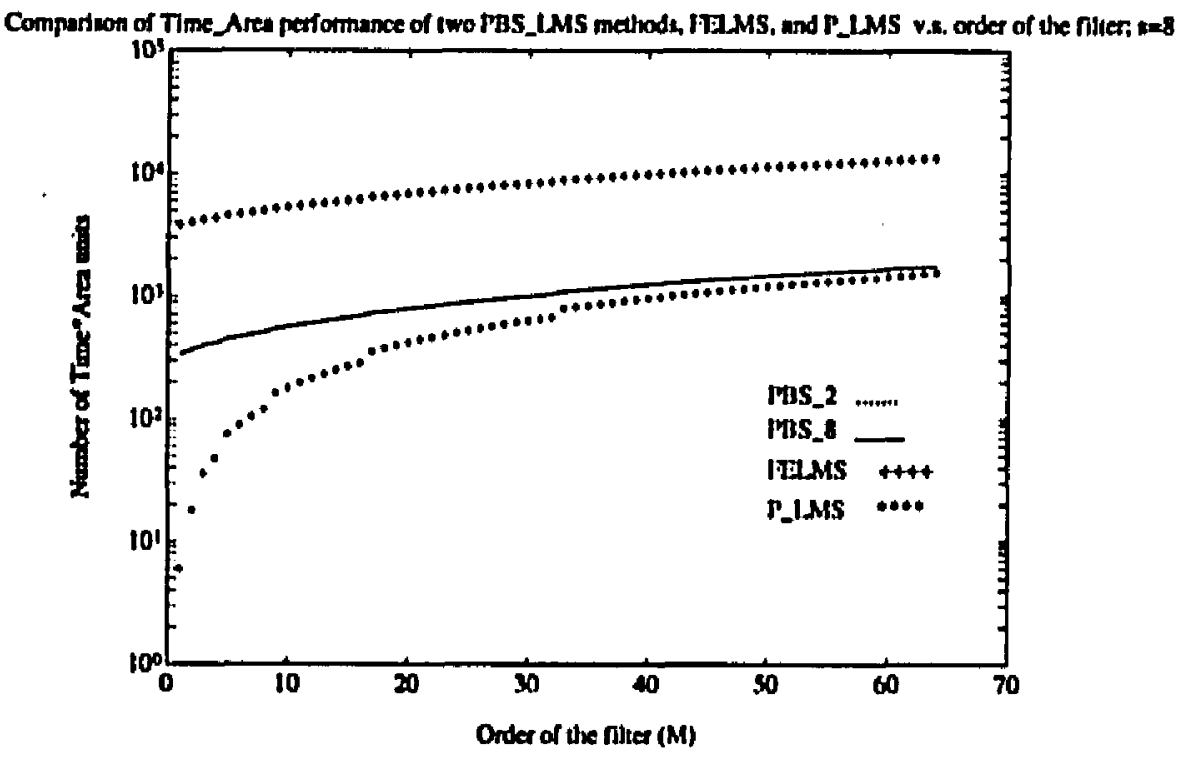

(c) 


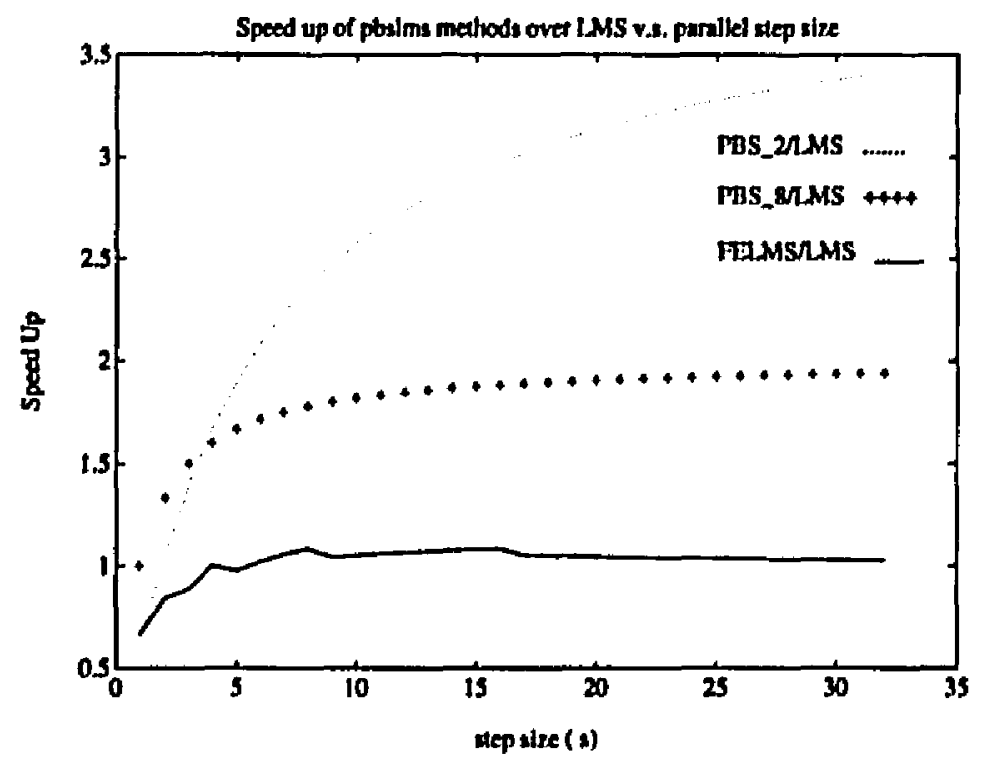

Fig. 55(a)

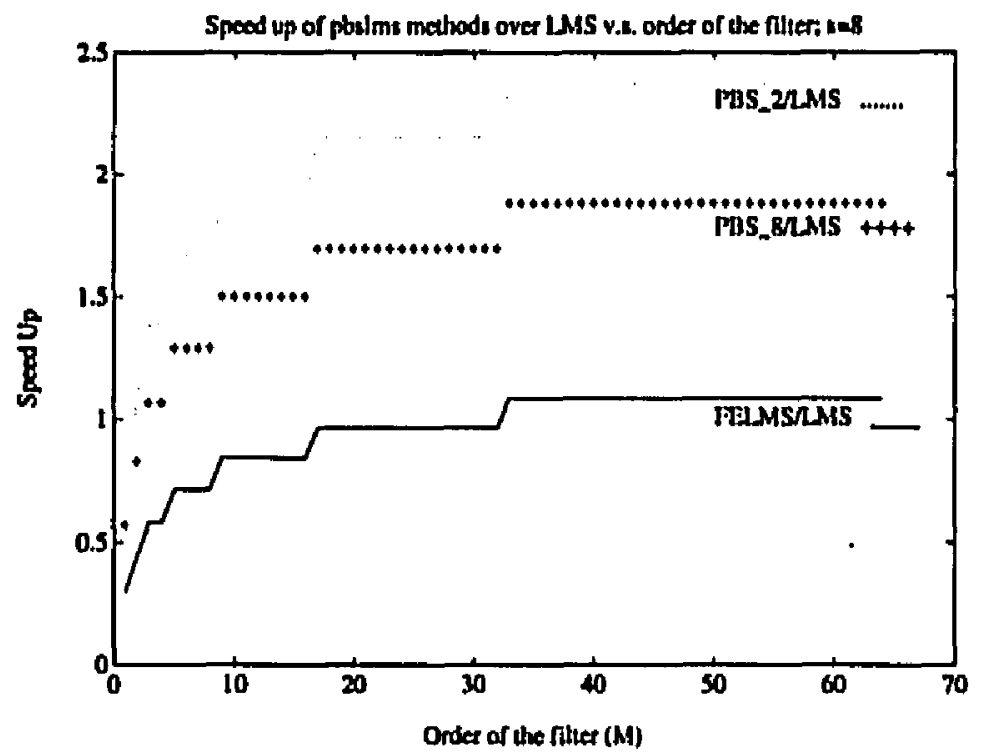

Fig. 55(b)

Figure 55: Comparison between the speed up of the PBS_2, PBS_8, and FELMS over P.LMS; (a) Speed ups versus step size $s$; (b) Speed ups versus order of the filter. 


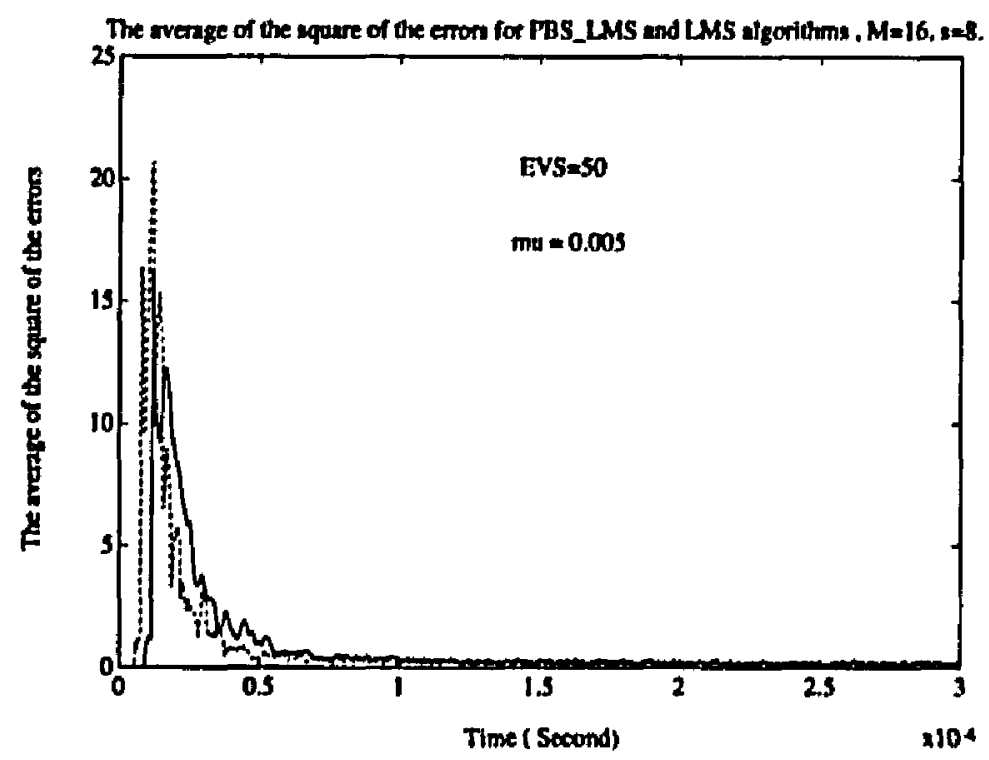

Figure 56: The average of the errors of two adaptive filters based on PBS.8 and PLMS algorithms

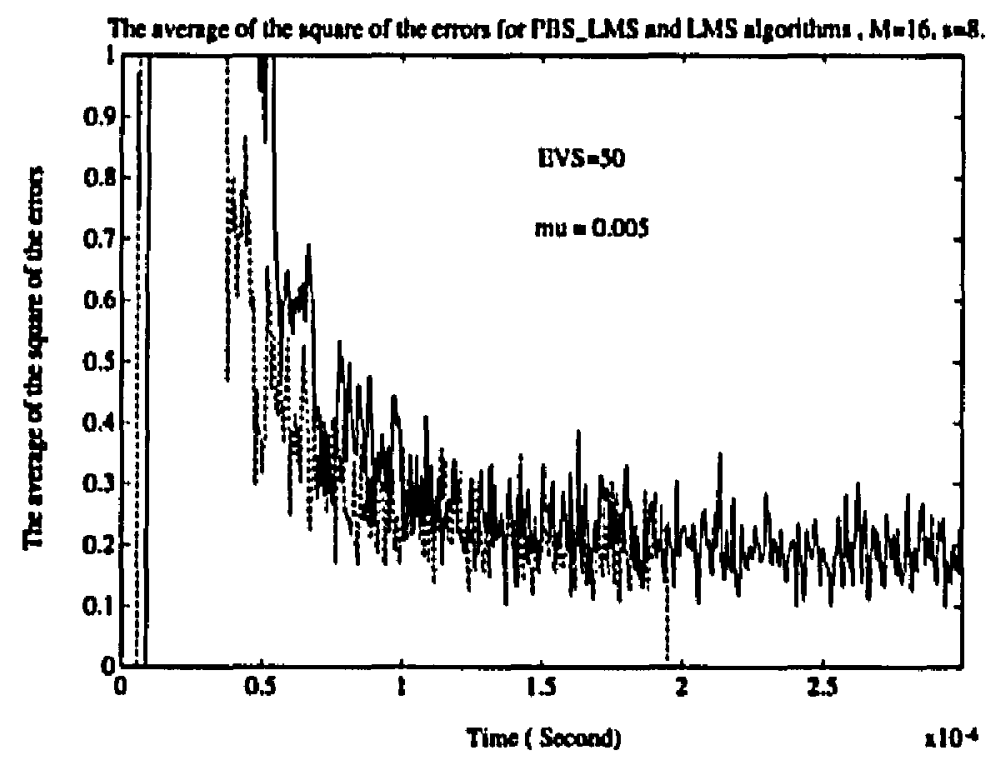

Figure 57: More details of the average of the errors of two adaptive filters based on PBS_.8 and P.LMS algorithms 


\section{CHAPTER VII}

\section{Architectural Implementation of PBS_LMS Algorithm Using VHDL}

In this chapter the results of the VHDL simulation of the arehitectures for the PBS.LMS algorithm are presented. The main aim of this simulation is to verify the functionality of the proposed algorithm for the adaptive filters, and also to show the regularity of this algorithm. The parallel algorithm, presented in the previous chapters can be implemented in VHDL for generic values of $s$, the parallel step size, and $M$, the order of the adaptive filter. Regularity is a very important factor for implementing this parallel algorithm in VLSI technplogy.

Using VHDL to evaluate the arehitecture is technology.independent and allows the designers to plan the circuit in the general form. Then, depending on the particular application and technology, the proper values for $s$ and $M$ can be chosen. That is, a paralle adaptive filter with larger order, $M$, or bigger parallel step size, $s_{1}$ can be obtained from the previous design. They use the same codes and change the generic values of the $s$ and $M$ and render another silicon layout for the desired adaptive filter.

The second goal of preparing the VHDL code for different PBS $\perp M S$ structures is to have comparisons between the theory, developed in the previous chapters, and the practice. These comparisons are in terms of time delay and unit count of the circuit.

The third intention of this VHDL simulation is to open the second phase of this 
research, physical implementation of a parallel adaptive filler. Starting from VHDL coding of PBS_LMS, one can use the present CAD tools to prepare the layout for such a parallel algorithm.

\subsection{General Description of the Parallel Adaptive Filer Chip}

Parallel Binary Structured LMS, PBS LMS, is used to simulate the behavior of an adaptive filter. We call this device Parallel Adaptive Filter Integrated Circuit and abbreviate it to PAFIC. Fig, 58 shows the overall layout of PAFIC and its inputs and outputs. Description of the input and output signals are summarized in Table 9.

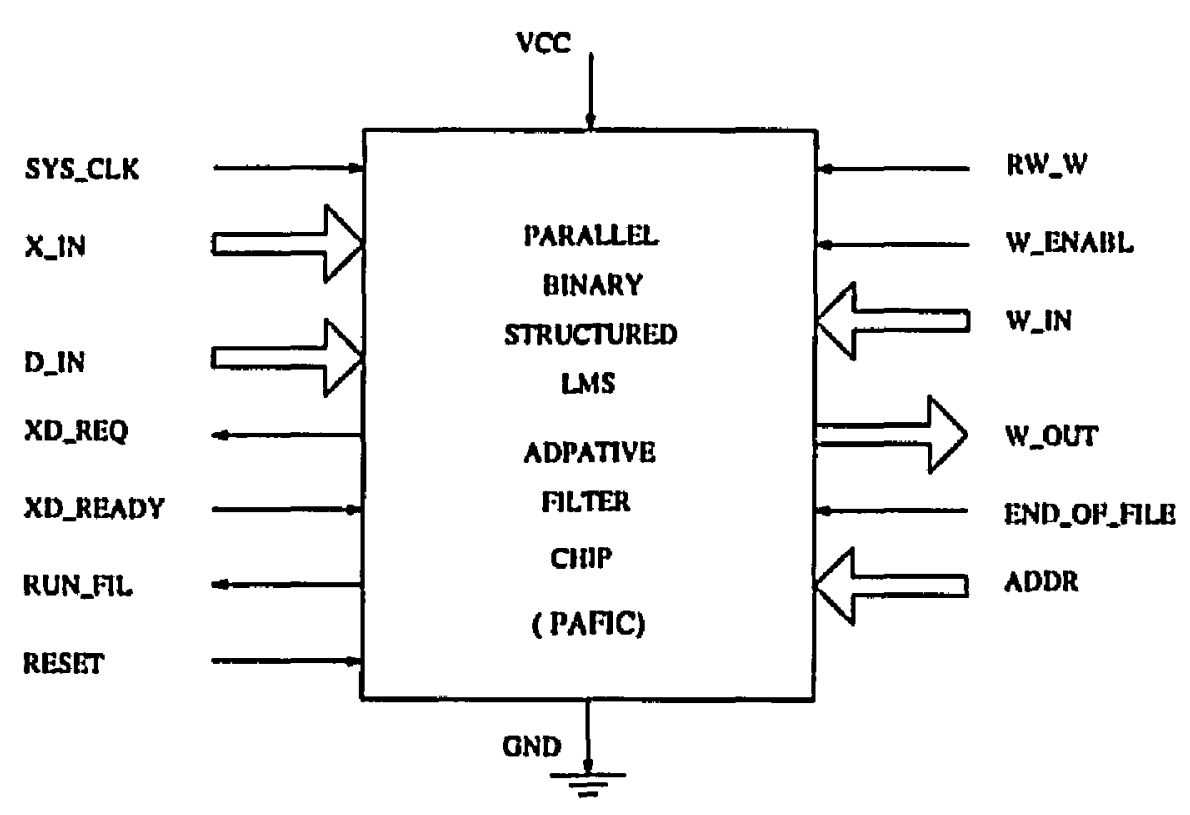

Figure 58: The over all layout of PAFIC

The block diagram and internal signals of PAFIC is depicted in Fig. 59. The internal structure of each block depends on which architecture of PBS.LMS is chosen. In the following sections, the detail structure of these blocks are shown. 
Table 9: Description of the input and output signals of PAFIC signal description

\begin{tabular}{|c|c|c|c|}
\hline Signal Name & IN/OUT & Type & Function \\
\hline sys_clk & IN & BIT & Clock of the system \\
\hline X.IN & IN & REAL & Input port to send $X$.values in \\
\hline D.IN & IN & REAL & Input port to send D.values in \\
\hline XD_REQ & OUT & BIT & $\begin{array}{l}0=\text { Send } X \text { 's and D's in, } l=\text { Halt } \\
\text { inputs }\end{array}$ \\
\hline XD.READY & IN & BIT & $\begin{array}{l}X \text { and } D \text { values are ready to be } \\
\text { read, active high }\end{array}$ \\
\hline RUN_FIL & OUT & BIT & Filter is running \\
\hline RESET & IN & BIT & Resel the chip, active low \\
\hline RW_W & IN & BIT & $\begin{array}{l}\text { I=read tap weights in, } 0=\text { writc } \\
\text { tap weights out }\end{array}$ \\
\hline W.ENABLE & IN & BIT & $\begin{array}{l}\text { Tape weights bus enable, ac- } \\
\text { tive high }\end{array}$ \\
\hline W.IN & IN & REAL & Tape wcights input bus \\
\hline W_OUT & OUT & REAL & Tape weights output bus \\
\hline end_of_file & IN & BIT & End of Test vector file \\
\hline ADDR & IN & INTEGER & Address Bus \\
\hline
\end{tabular}

The controller of the PAFIC produces the handshaking signals for the test bench. The controller also controls the flow of data to and from the device. The control signals on the controller bus are given below.

-- signals on the controller bus of the PAFIC chip

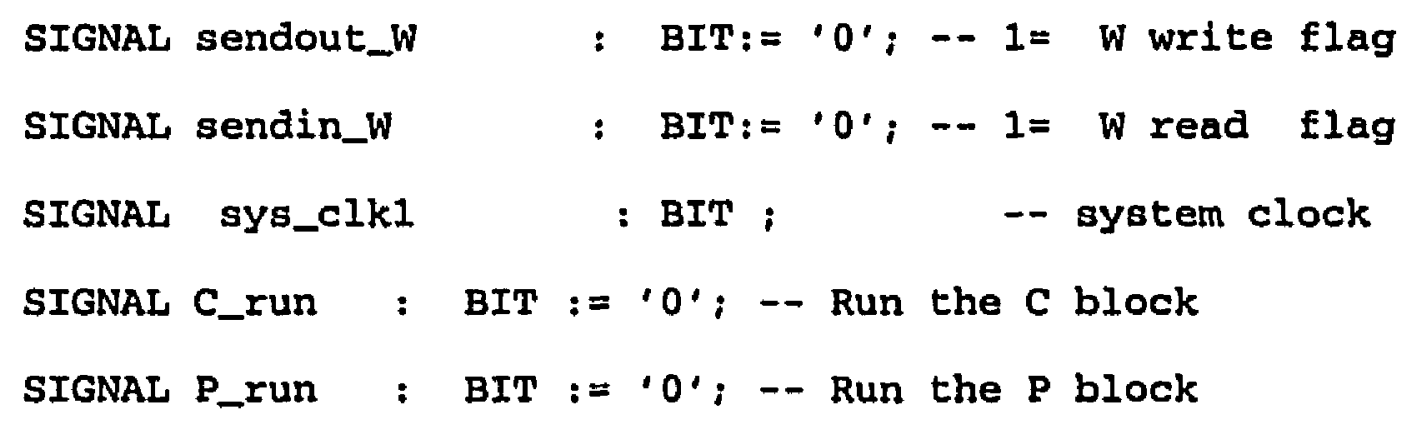




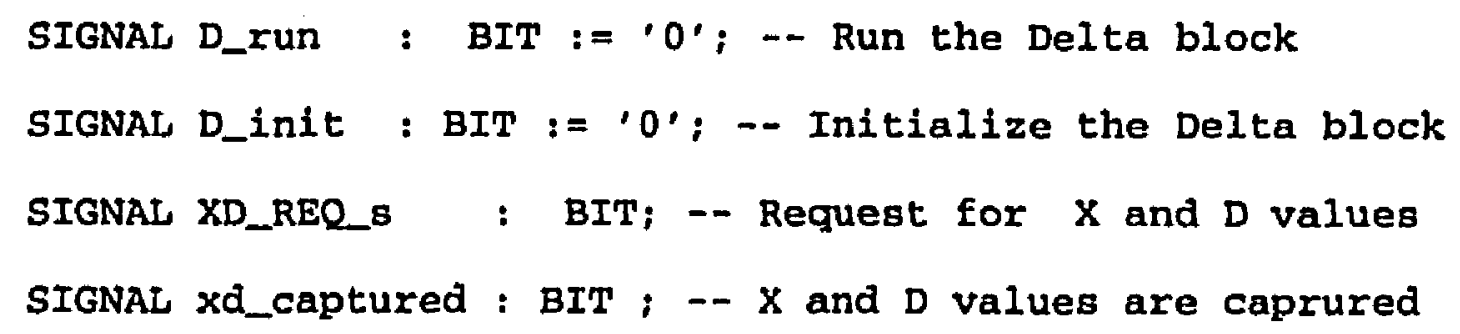

Using the standard cells in a design decreases the layout time. Standard cells are units whose VHDL codings, or their layouts, are designed and stored in the design library. Custom design units usually occupied a large portion of a design project. In order to avoid custom design units in our circuit, we tried to use the basic and standard cells. In our VHDL simulation four basic cells are used. These cells are adders, multipliers, multiplexors, and latches. The more complexed units are bulit using these four basic cells.

\subsection{Test Bench for the PAFIC}

The block diagram of a test bench to verify the funclionality of the PAFIC is shown in Fig. 60. As discussed in the previous chapters, in order to test an adaptive filter two random processes are required. One random process is the input to the adaptive filter. And the other random process is the desired output of the filter. Using the procedure described before, a sequence of random process is generated and stored in the X.value memory on the test bench. The corresponding random process produced by the unknown system using $X$.values as the input, is stored in the D_value memory on the test bench. $X_{\text {_values and }}$ D.values are the test vectors for the circuit.

The controller of the test bench produces the handshaking signals. It also controls the flow of data to and from the device under the test. System clocking is generated in the 


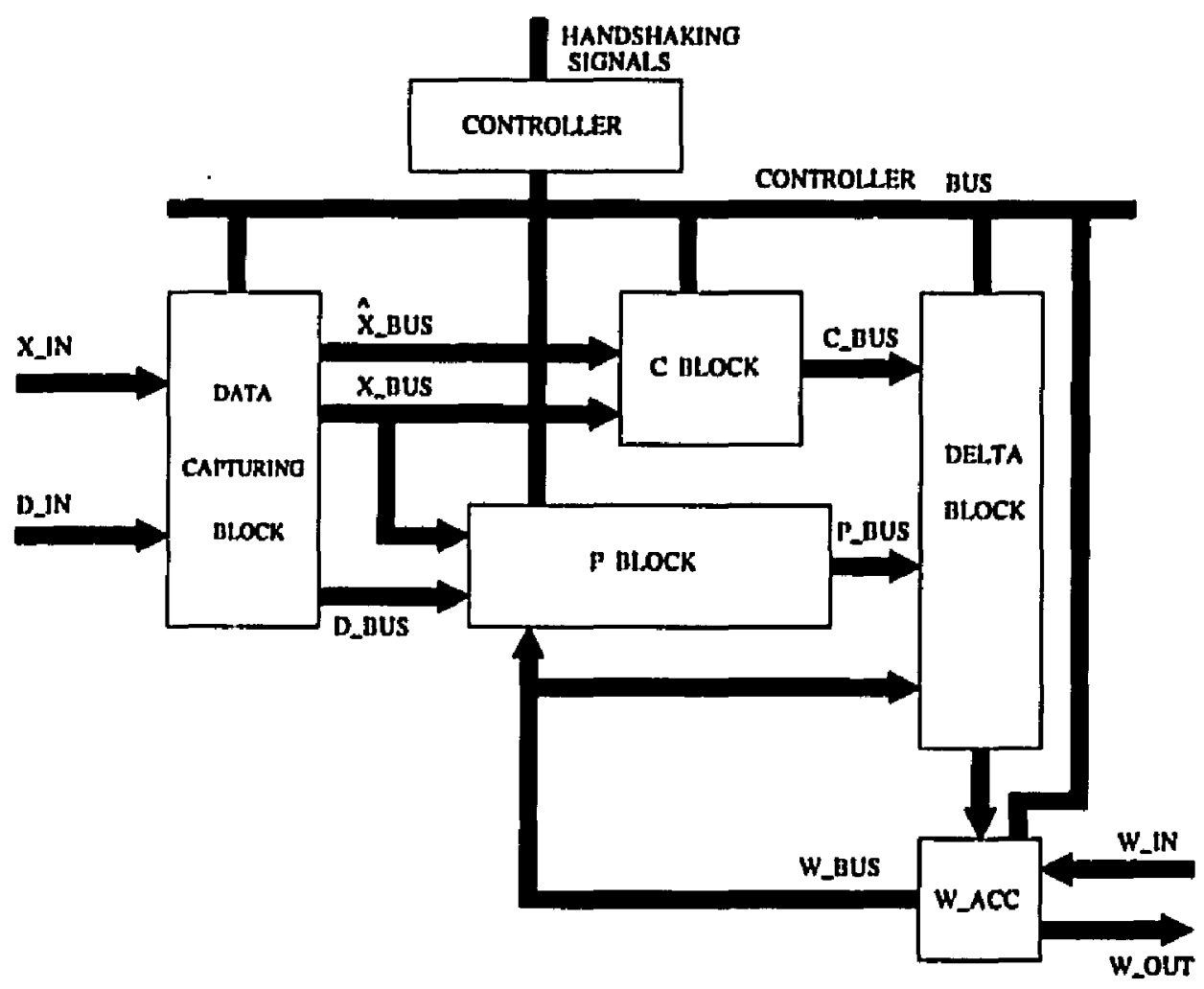

Figure 59: Blocks and Signals inside PAFIC

SYS_CLK block. It synchronizes the actions of the different parts of the test bench and the device under the test, PAFIC. The control bus of the test bench contains the following signals.

-- controller signals
SIGNAL Reset
: BIT :=10'; -- Active low,
SIGNAL W_ENABLE
: BIT := $0^{\prime} ;$-- Active high
SIGNAL RW_W
: BIT := 10 ' ; -- Active high
SIGNAL RUN_EIL
: BIT :='1', -- Active high 
SIGNAL XD_REQ :BIT;-- $0=$ Send $X^{\prime} s$ and $D^{\prime} s$ in, $1=$ Halt inputs SIGNAL XD_READY:BIT;-- $0=$ Send $X^{\prime} s$ and $D^{\prime} s$ in, $1=$ Halt inputs SIGNAL end_of_file:BIT :='0'; -- end of test vector files

In the test bench, generic values for the paralle step size, the order of the filter, the gradient step size, basic cells delay times, and the system clock period are defined.

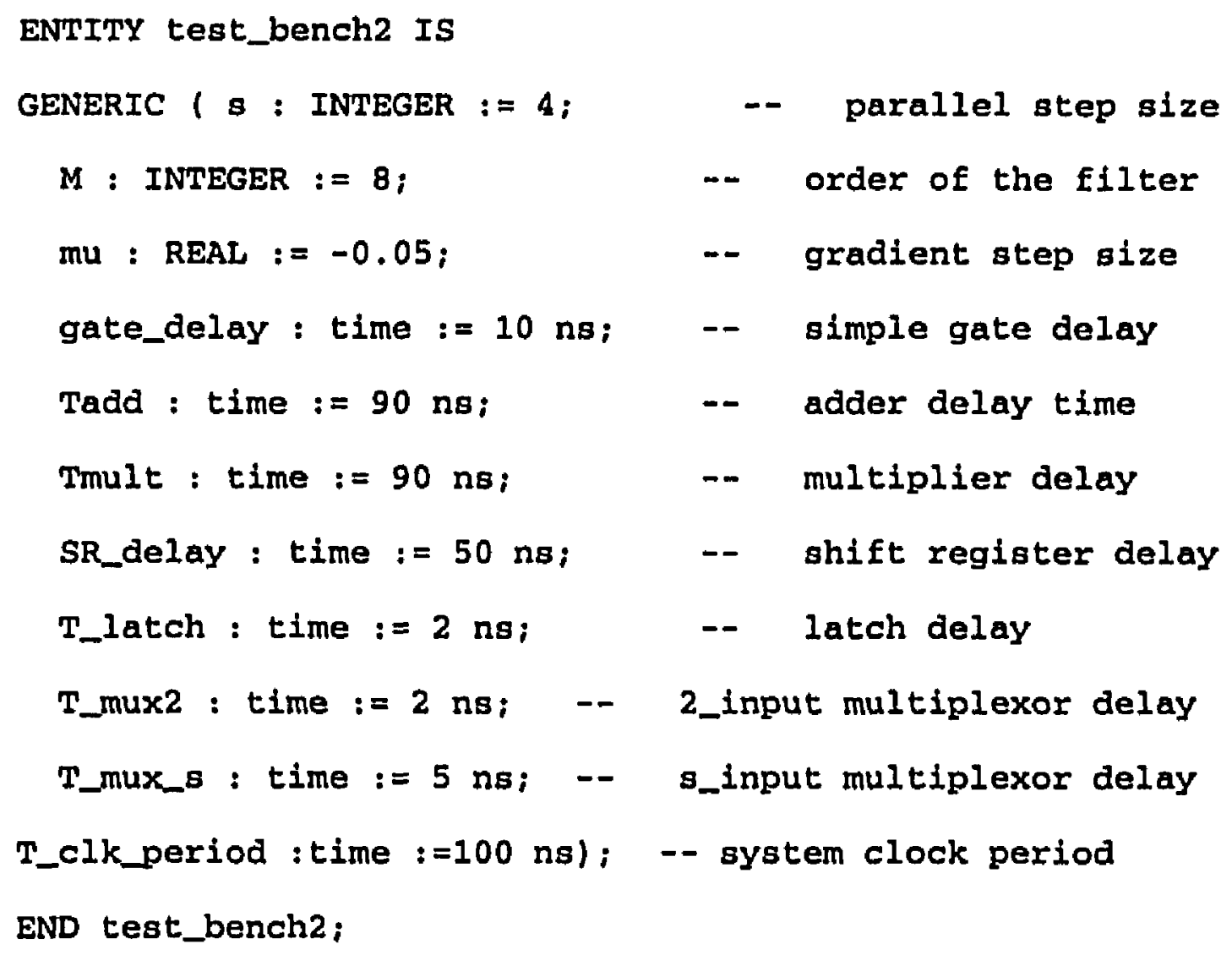

The flow of the data on the test bench are on the four data buses and the address buses. The address bus carries the value of the address. The other buses are for $X$ values, $D$ values, tap weights to the device, and tap weights out of device. The buses of the test bench circuit are defined as follows 
-- test bench buses

$\begin{array}{lll}\text { SIGNAL X_IN } & \text { : REAL } & :=0.0 ; \\ \text { SIGNAL D_IN } & : \text { REAL } & :=0.0 ; \\ \text { SIGNAL W_IN } & : \text { REAL } & :=0.0 ; \\ \text { SIGNAL W_OUT } & : \text { REAL } & :=0.0 ; \\ \text { SIGNAL ADDR } & : \text { INTEGER } & :=1 ;\end{array}$

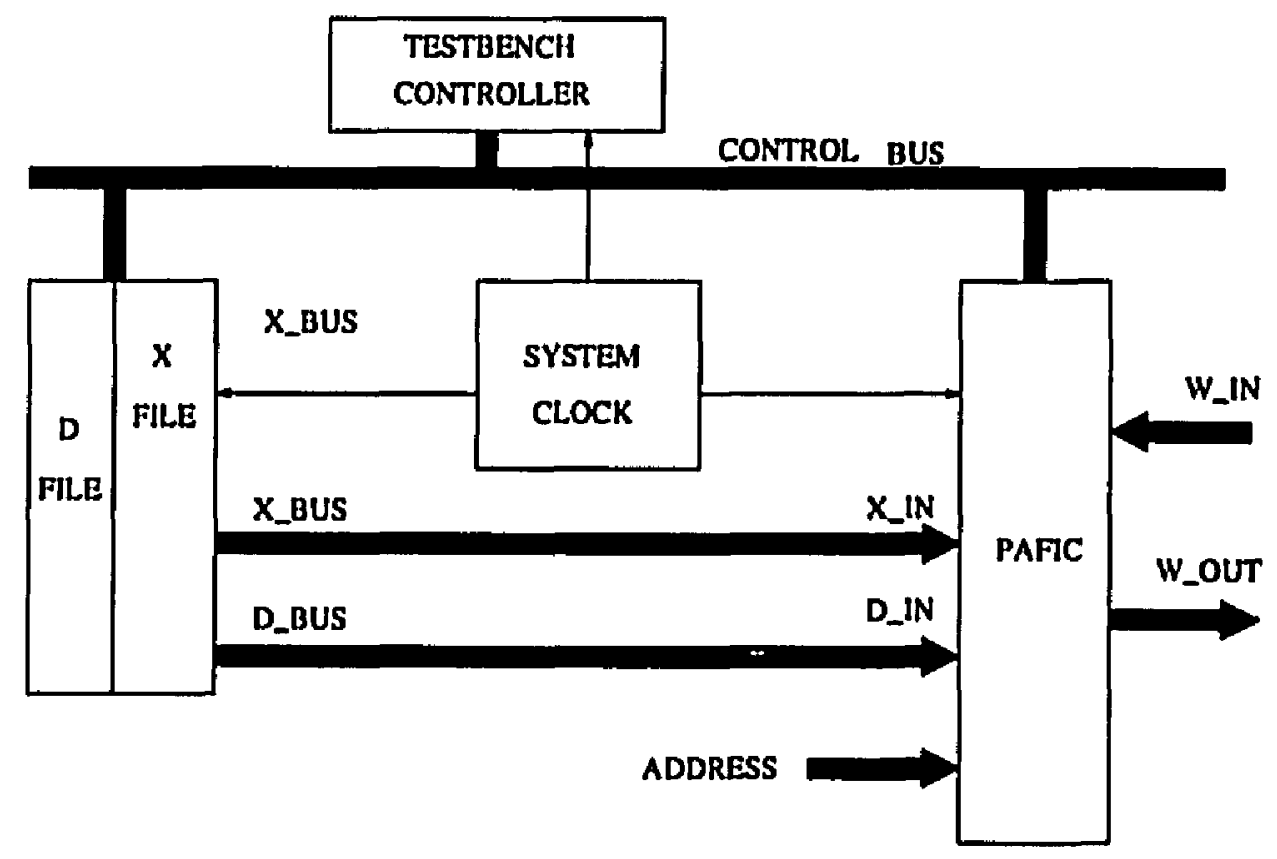

Figure 60: Blocks of the test bench of PAFIC

\subsection{PBS_2 implementation}

As investigated in the previous chapters, PBS_2 is one of the optimum structures for PBS_LMS algorithm. In this section the details of VHDL implementation of this architec- 
ture is presented. Refer to section 5.5, PBS_2 is the combination of C2, P2, and D5,

$$
\mathrm{PBS} 2=\{C 2, \mathrm{P} 2, \mathrm{D} 5 \mathrm{\}}
$$

The $\mathrm{C} 2$ architecture is implemented in the $\mathrm{C}$ block, $\mathrm{P} 2$ in the $\mathrm{P}$ block, and D5 in the $\Delta$ block.

\subsubsection{C2 for the $\mathrm{C}$ block}

In $\mathrm{C} 2$ configuration of the $\mathrm{C}$ block all $c$ 's are calculated in parallel, using the $c$ 's from the previous input block,

$$
\begin{aligned}
c_{n e w}(i, j)= & c_{\text {old }}(i, j)+\sum_{k=0}^{+-1} x^{*}(n+i-k) x(n+j-k)- \\
& \sum_{k=0}^{-1} x^{*}(n+i-k-M) x(n+j-k-M) \\
& \text { for } i=1, \ldots, s-1, \text { and } j=0,1, \cdots, i-1 .
\end{aligned}
$$

C2 requires $s(s-1)$ multipliers and $\frac{(1-1)(e+4)}{4}$ adders, when the results of the summations are saved. The minimum time for calculating the new $c$ 's is

$$
T_{C 2}=T_{m u l t}+\left(1+\left[\log _{2} s\right]\right) T_{\text {add }}
$$

To implement the above structure, the $\mathrm{C}$ block has a structure similar to one is shown in Fig. 16. The configuration of the array of multipliers and registers in the $\mathrm{C}$ block, for a parallel adaptive filter with step size $8, s=8$, is shown in Fig. 61. The elements of lower triangle in Fig. 61 (a) are registers, in which the results of previous multiplications are saved. Fig. 61(b) shows one of the elements used in the parallelogram array of multipliers of Fig. 61(a). 


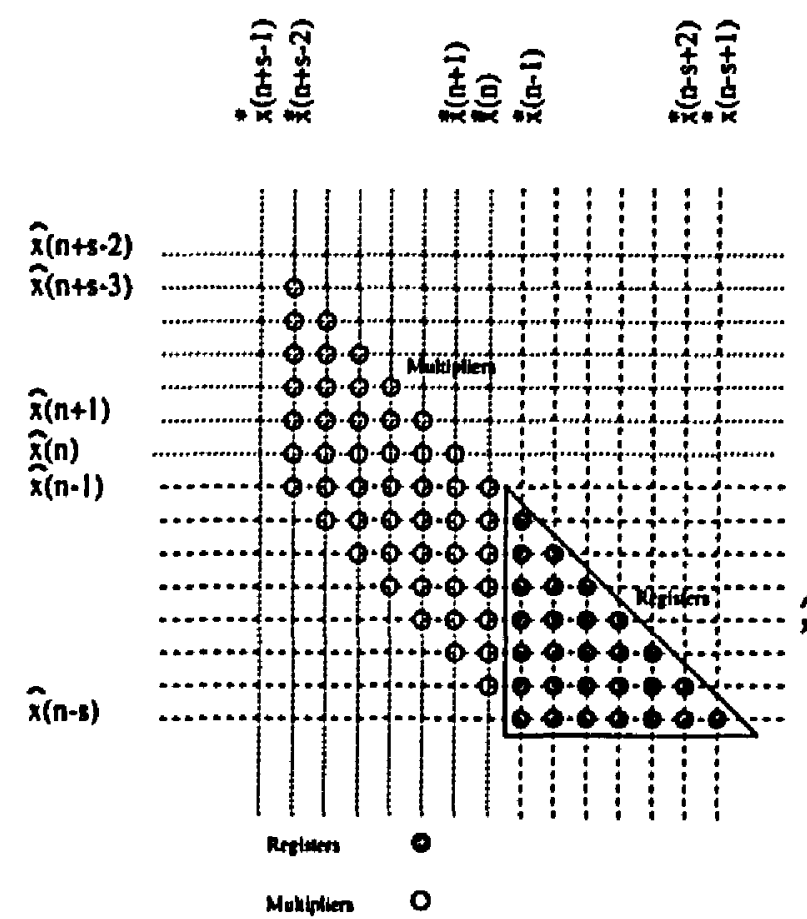

(a)

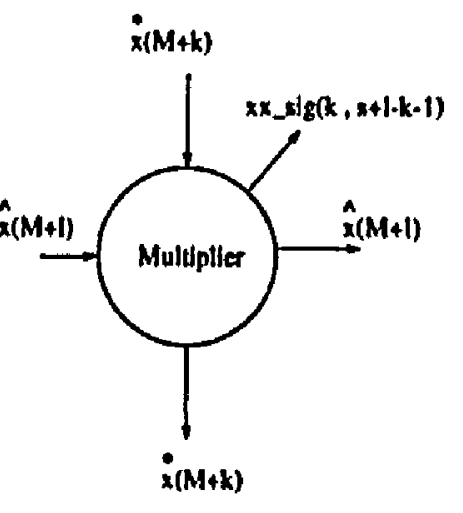

(b)

Figure 61: The Configuration of Multipliers in the $\mathrm{C}$ Block for circuit with parallel step size equal 8; (a) Multipliers and Registers configuration; (b) One Multiplier.

Figure 62 shows the detail structure of one of the s_number_adder and an extra subtractor and adder shown in Fig 16. The name of units and signals are the same as they used in the VHDL codes.

\subsubsection{P2 for the P block}

When $\mathrm{P} 2$ architecture is used to implement the $\mathrm{P}$ block, for each $p(s i), \frac{M I}{2}$ adders and $M$ multipliers are needed, provided multiplexors in the inputs of first half of adders. The $P$ block requires a total of $s\left(\frac{M}{2}+1\right)$ adders and $s M$ multipliers. Figure 63 depicts the circuit for one of $p(s i)$ 's for a filter of order of $M=16$. 


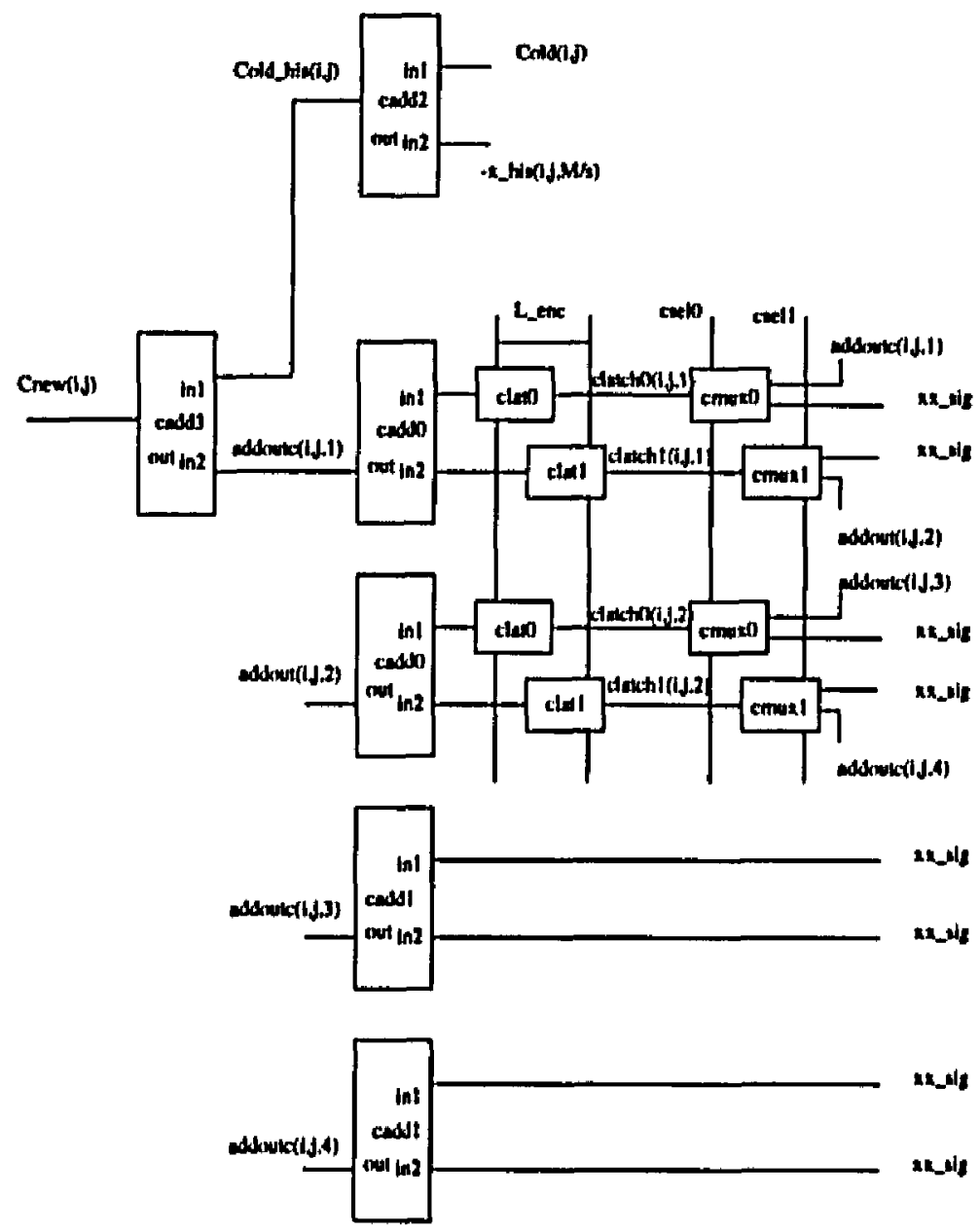

Figure 62: Adders, Latches, and Multiplexors required in the $s$ number adders of the $\mathrm{C}$ block for each $c$ coefficient, $s=8$.

\subsubsection{D5 for the $\Delta$ block}

In D5 method, $g$ 's are calculated recursively, Start from $g(0)=p(0)$, then calculate the other $g(s i)$ 's as

$$
g(s i)=p(s i)+\sum_{k=0}^{\operatorname{si}-1} c(s i, k) g(k) \quad \text { for } \quad s i=1, \ldots, s-1 .
$$




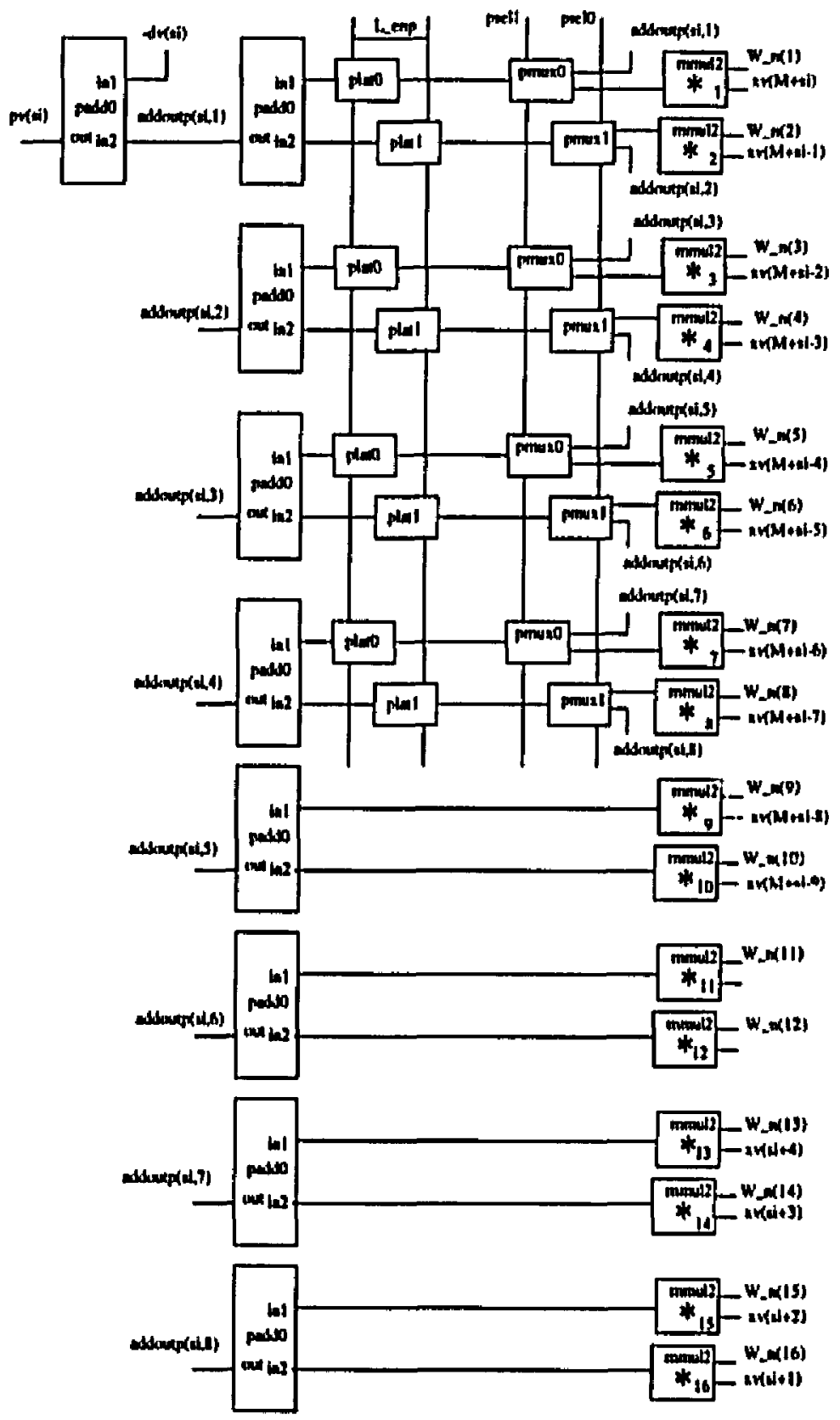

Figure 63: Circuit to calculate one of $p(s i)$ 's in the $\mathrm{P}$ block, using $\mathrm{P} 2$ architecture. 
Multiply $g(s i)$ to its corresponding vector, $\hat{\mathrm{x}}(n+s i)$, to get $\mathrm{dwv}(s i)$ vector

$$
\mathrm{dwv}(s i)=g(s i) \hat{\mathbf{x}}(n+s i)
$$

Then, sum up all dwv(si), si $=0,1, \ldots s-1$, vectors to obtain $\Delta \mathrm{w}(n+s)$

$$
\Delta \mathrm{w}(n+s)=\sum_{s i=0}^{s-1} \mathrm{~d} w \mathrm{v}(s i)
$$

Finally add $\Delta w(n+s)$ to $w(n)$ to produce $w(n+s)$,

$$
w(n+s)=w(n)+\Delta w(n+s) .
$$

The systolic structured which calculates the g's is shown in the lower part of Fig. 64 . The upper part of Fig. 64 depicts the circuit which calculates $d w v(s i), s i=0,1, \ldots s-1$, $\Delta w(n+s)$, and $w(n+s)$ for an adaptive filter of order 2 . The complete circuit for the upper part of Fig. 64 for an adaptive filter of order $M$ is depicted in Fig. 65.

\subsubsection{Timing Diagrams of the PBS 2 simulation}

The results of the VHDL simulation are shown in the four following figures. Timing diagram of the circuit for a ordinary filtering cycle is shown in Fig. 67. It shows the control and handshaking signals between the device and the test bench circuit. The effect of the RESET signal at the beginning of the process is shown in Fig. 66. The RESET signal sets the values of all registers in the device to zero.

The timing diagram for reading a set of tap weights values, by the device, is shown in Fig. 68. W.READ cycle is initiated by the test bench when tap weights of the adaptive filter are to be set to some predefined values. In the W.WRITE cycle, requested by the test bench, the calculated values of the tap weights are written on the W.OUT bus. Fig. 69 depicts the timing diagram of a W_WRITE cycle. 


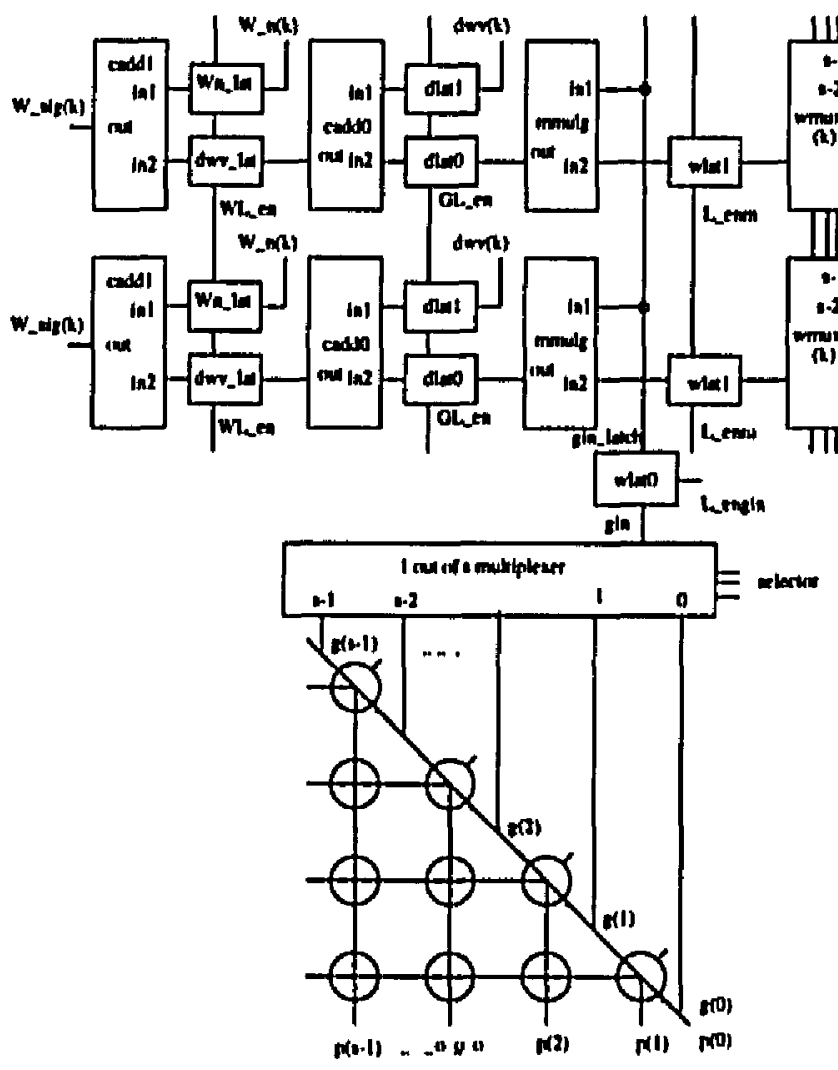

(c)

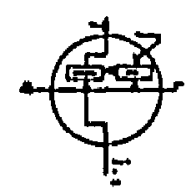

(a)

Nom_diegrad eluneter

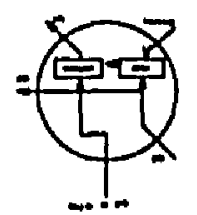

(b) Daspinal flenew

Figure 64: The structure of the $\triangle$ block usinf D5 architecture; (a) A Diagonal element of the lower part; (b) A Non_diagonal element of the lower part; (c) Over all recursive structure - of the $\Delta$ block, calculating $g(s i)$ 's, dwv(si)'s, and $w(n+s)$ 's. 

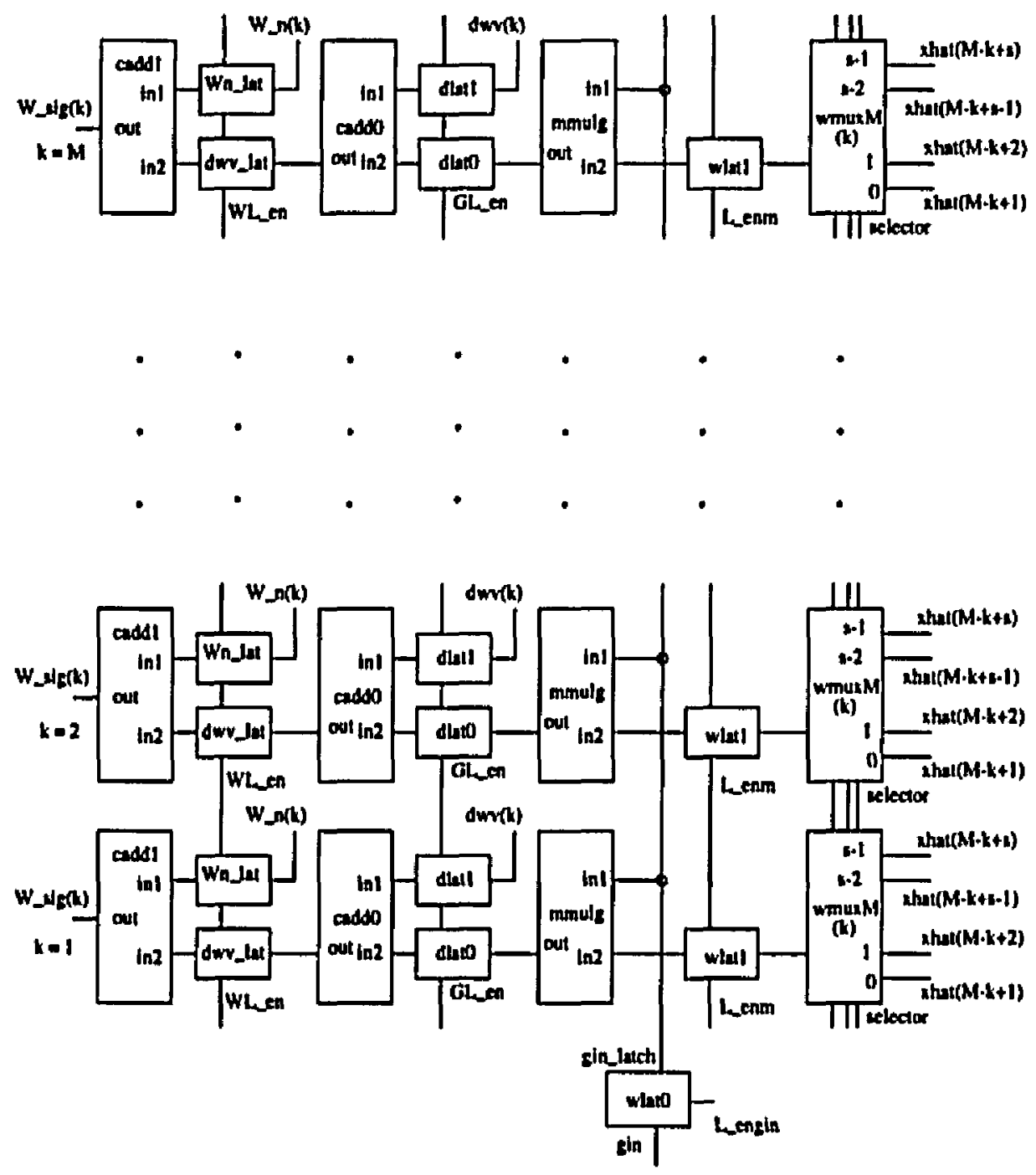

Figure 65: The circuit which calculates $\mathrm{dwv}(s i), s i=0,1, . . s-1$ and $w(n+s)$ in the $\Delta$ block for an adaptive filter of order $M$. 


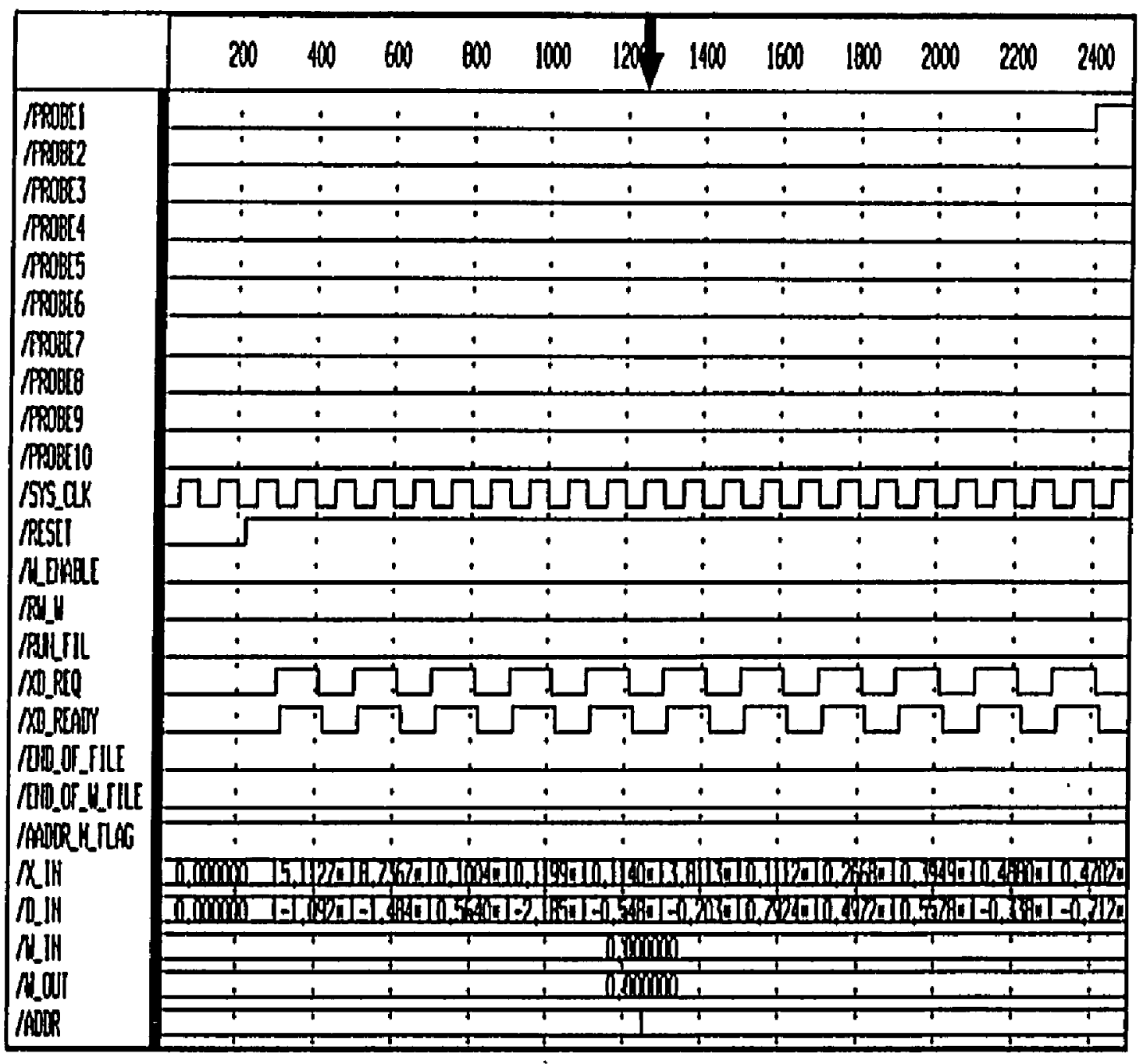

Figure 66: Timing dingram of a RESET cycle 


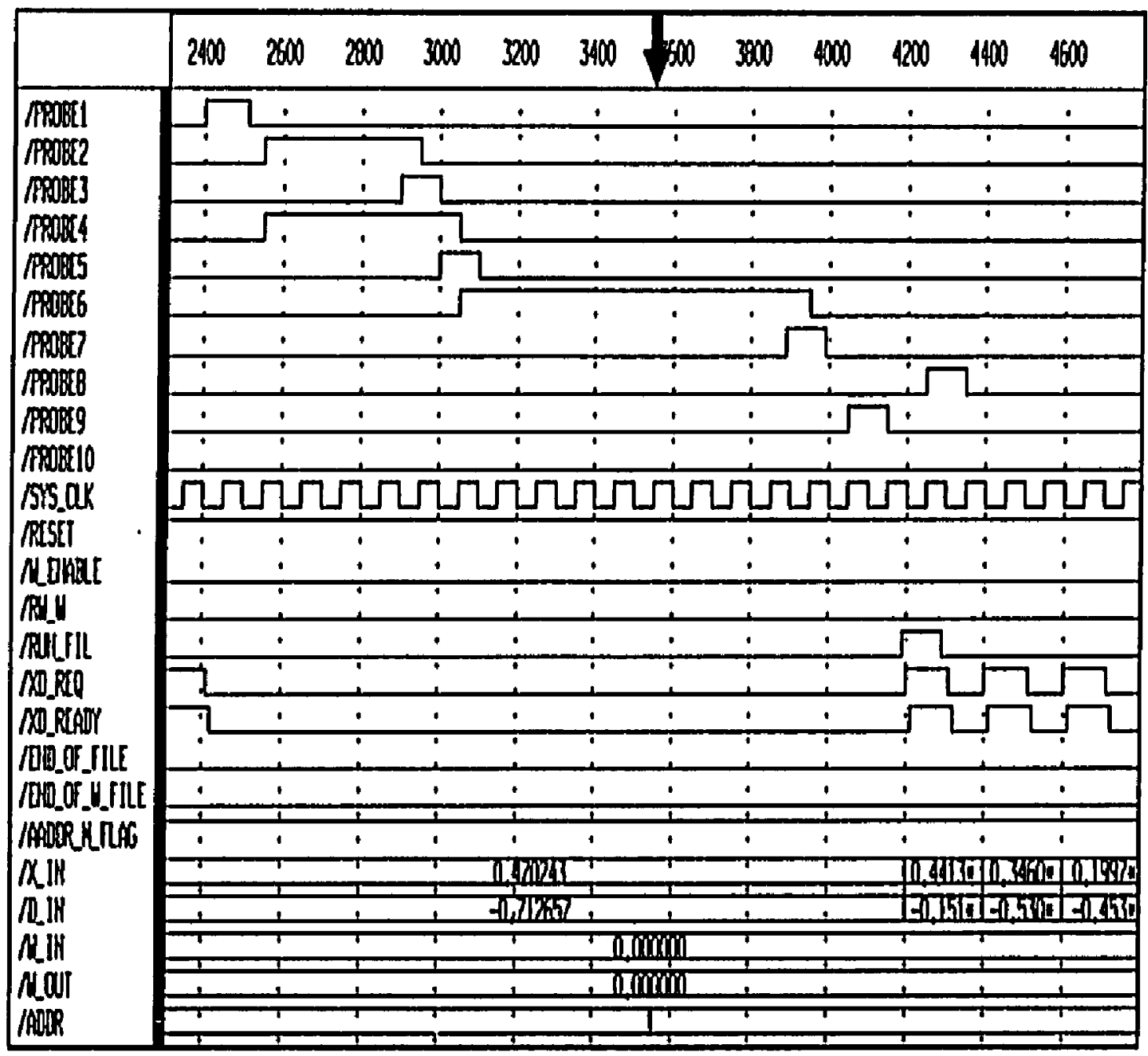

Figure 67: Timing diagram of a FILTERING cycle 


\begin{tabular}{|c|c|}
\hline & 45050005550550055505200555055005505555055005505500 \\
\hline $\sin$ & 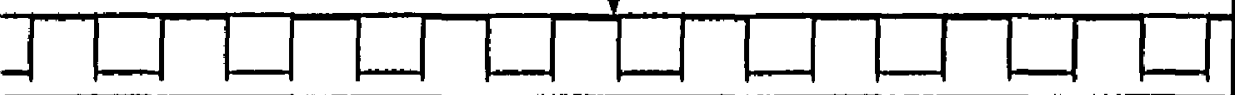 \\
\hline & \\
\hline & \\
\hline & \\
\hline & . \\
\hline & $\vdots$ \\
\hline IRAOY & $\therefore$ \\
\hline 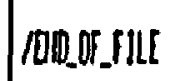 & $::$ \\
\hline Wult & 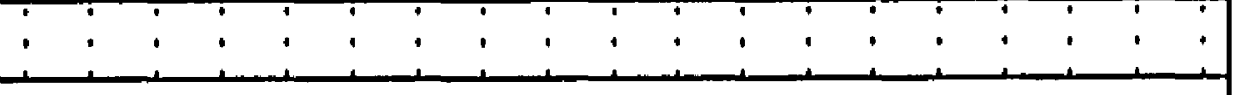 \\
\hline Rino & 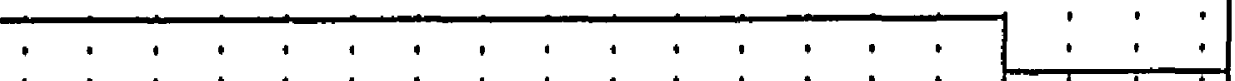 \\
\hline & $i: i_{i} i_{i}$ \\
\hline & $-0,0,14251 \cdot ;$ \\
\hline Ill & $\bar{i}: \vdots$ \\
\hline iar & 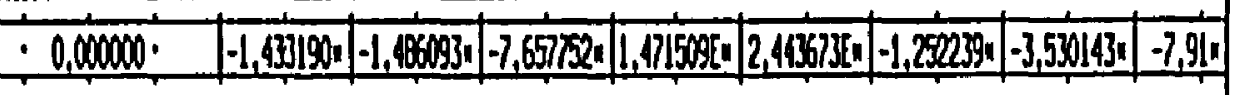 \\
\hline & 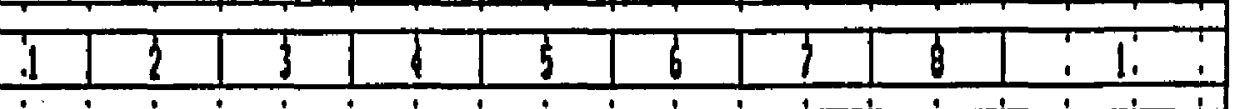 \\
\hline
\end{tabular}

Figure 68: Timing diagram of a W.WRITE cycle 


\begin{tabular}{|c|c|}
\hline & 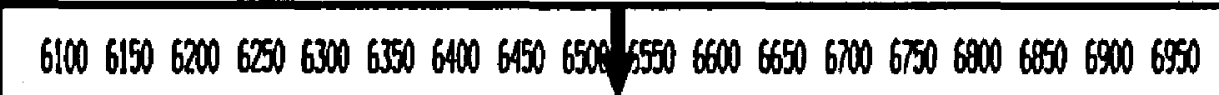 \\
\hline ts ak & 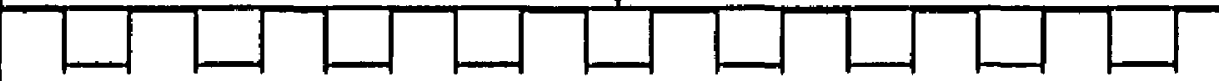 \\
\hline & $\%$ \\
\hline ald & _ \\
\hline & \\
\hline Hifl & \\
\hline 0850 & : : : \\
\hline StâM & 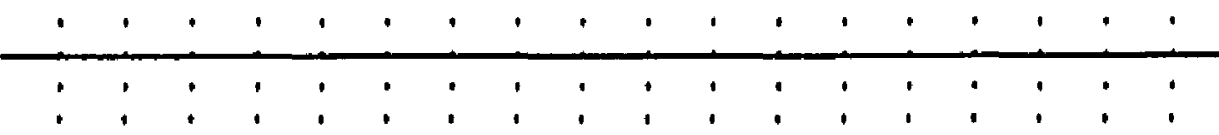 \\
\hline EIIIE & $\vdots: \vdots: \vdots \vdots: \vdots \vdots:$ \\
\hline MRRMar & - \\
\hline IIt & 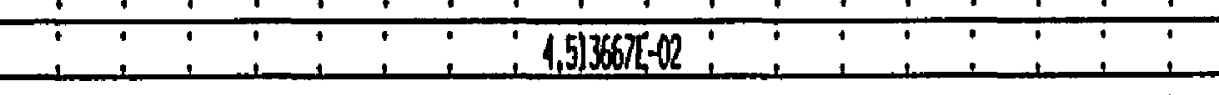 \\
\hline III & 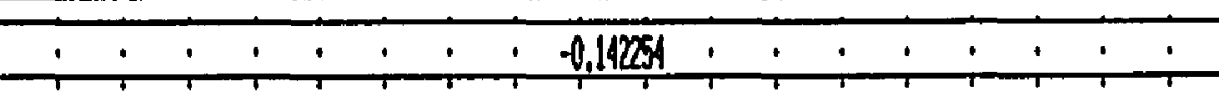 \\
\hline II & 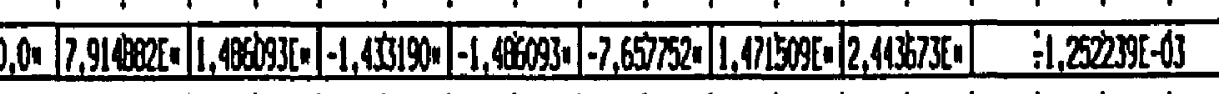 \\
\hline dir & 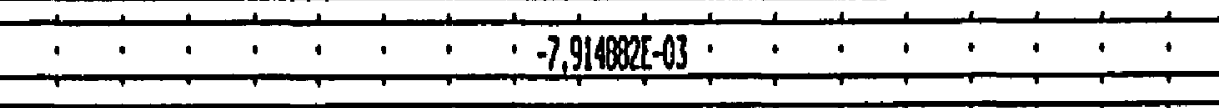 \\
\hline & \begin{tabular}{ll|l|l|l|l|l|l|l} 
& 1 & 3 & 1 & 5 & 6 & 1 & 6 & $\vdots$ \\
\end{tabular} \\
\hline
\end{tabular}

Figure 69: Timing diagram of a W.READ cycle 


\section{CHAPTER VIII}

\section{Conclusion and Future Works}

In this dissertation, a parallel algorithm for adaptive filters suitable for VLSI implementation is presented. Based on a direct relation between the binary presentation of numbers with $s$ digits and the terms needed to calculate the difference vector, a parallel structure for an adaptive filter is obtained which is an exact representation of the original LMS algorithm. Using this parallel algorithm, the tap weights of an adaptive fitter at time $n+s$ are calculated in terms of the tap weights at time $n$, inputs from time $n$ to time $n+s-1$, and desired outputs from time $n$ to time $n+s-1$.

This research is partitioned into three major steps. The first step is to develop a parallel algorithm for adaptive filters. The parallel binary structured LMS is derived from sequential LMS algorithm. The area, time, and the area_time product are used as measures of merit in evaluation of the algorithms. Component count is used as appropriate to estimate the area. Correspondingly, component delay is used to estimate the time. The algorithms are also evaluated in regards to the ability to use regular and modular structures in a VLSI implementation. The ability to partition the VLSI architecture into major sub_elements is also evaluated.

The second step of the research is to evaluate the impact of the different parallel and pipelined techniques on the time and area of each block of the PBS L.MS based adaptive 
filter. In order to achieve the maximum possible speed, with minimum number of units, the use of the pipelining in the implementation of the parallel structure is investigated. Since pipelining increases the design time, its effect on the design of each block and on the performance of the adaptive filter is evaluated.

The third step of our research is to evaluate the presented parallel binary structured algorithm for a VLSI impiementation. The most time and area-time efficient architectures are mapped to a corresponding VHDL architectures. VHDL is used to verify and validate the arehitectures at a high level of abstraction using a behavioral model. The VHDL description is modified to a structural description employing the regular and modular structures identified in the first step.

The proposed parallel algorithm is a simple and versatile algorithm for adaptive filters, suitable for implementation on a VLSI chip. The presented regular and modular algorithm is such that it is capable of enhancing the number of paraliel steps, as well as the order of the filter, without significantly increasing the complexity of its VLSI circuit. the performanec of the proposed binary structured LMS algorithm is compared to the performance of the parallel LMS and Fast Exact LMS algorithms.

A comparison between the performance of the proposed binary structured LMS algorithm and the performance of two other parallel LMS algorithms shows that the PBS $\perp M S$ converges two to five times faster than the others, without increasing the time_area criteria.

\section{Future Works}

Continuation of this research can lead to a parallel adaptive filter chips. It is desirable for the algorithm to map to a VLSI architecture that uses structures that are both regular and 
modular. The VLSI architecture should allow either modular expansion of the order of the filter or the degree of parallelism obtainable.

Pipelining in execution of each operation, like addition or multiplication, and its effect on the performance of the PBS $\perp M S$ can be evaluated.

A general purpose adaptive filter chip based on the PBS.LMS algorithm can be designed. Each chip contains a fixed_number_of_tap adaptive filter. It is desired that the parallel adaptive filter chips be cascadable in order to obtain a larger adaptive filter. It is also desired to design a cascadable chip to be able to extend the number of parallel steps. Extending the order of the filter seems more realizable than extending the parallel step size. Therefore, the work on cascadability of the adaptive filter chips can be focussed on increasing the order of the filter for a fixed parallel step size.

A set of chips for a general purpose adaptive filter capable of a fast performance can be designed. A significant issue in this step is the partitioning of the architecture into components that may be mapped to an optimum number of dies, keeping chip count and inter-chip communication requirements to a minimum. 


\section{Appendix A}

\section{Time and area estimation of the Fast Exact LMS algorithm}

Authors in [24] present an algorithm, called Fast Exact LMS or FELMS, which obtains the results of the LMS algorithm in a parallel manner. The total number of multiplications and additions per iteration in their proposed algorithm is less than the total number of multiplications and additions requires in the sequential LMS algorithm.

To execute FELMS with a block size of $N$, first calculate $\frac{N(N-1)}{2}$ elements of $s_{i}\left(n_{N}+\right.$ $i+1)$, for $i=1,2, \ldots, N-1$, using equation 26 and 27 of [24]. Then form $N-1$ lower triangular $N \times N$ matrices of $G_{i}(n)$, using $s_{i}(k)$ elements. Diagonal elements of all these matrices are one. The product of these $N-1$ matrices is another $N \times N$ lower triangular matrix, called $G(n)$ in equation 21 of [24]. Then $N$ elements of the error vector is calculated using equation 28a. And tap weights of the adaptive filter are updated through equation $28 b$.

The authors of [24] have concentrated their effort on complexity performance of their proposed algorithm. They compare the complexity of FELMS with sequential LMS and block LMS, described in [29] and [13], respectively. The performance of a parallel algorithm is not complete unless a time and area performance of that algorithm is investigated. Authors of [24] did not evaluate their proposed algorithm with time, area, and time-area criteria. Our estimation of these three performance indices for FELMS follows. 
In order to estimate the above indices, time, area, and time_area, for the Fast Exact LMS algorithm, following steps and assumptions are considered. FELMS requires calculation of all the terms of equations 26,27,21, and 28 in [24], for an adaptive filter of order $L$ and a block size of $N$.

$N(N-1)$ multipliers and $N^{2}+N$ adders are needed to calculate the $\frac{N(N-1)}{2}$ elements of equations 26 and 27 with a time delay of

$$
T_{(1) 26,27}=T_{\text {mult }}+\left(N-1+\left\lceil\log _{2} N\right\rceil\right) T_{\text {add }}
$$

However with the same number of multipliers, $N(N-1)$, the number of the adders can be reduced to $N$ with a time delay of

$$
T_{(2) 26,27}=T_{m u l t}+4(N-1) T_{\text {add }}
$$

To calculate $G(n), N-1$ lower triangular $N \times N$ matrices are to be multiplied. Using the parallel systolic array structure, described in [28], each matrix multiplication can be obtained with $4 N T_{\text {mult }}+4 N T_{\text {add }}$ time delay. In this systolic array, there are $N^{2}$ processor elements, PE's. Ench PE has one multiplier and one adder. The time required to calculate $G(n)$ is

$$
T_{\mathrm{lG}(n)}=4 N(N-1)\left(T_{m u l t}+T_{\text {add }}\right) .
$$

Employing a tree structure with $N^{3}$ processor elements, $G(n)$ can be obtained with a time delay of

$$
T_{2 G(n)}=4 N\left\lceil\log _{2}(N-1)\right]\left(T_{\text {mult }}+T_{\text {add }}\right) .
$$

Since $G(n)$ is the product of $N-1$ lower triangular matrices, it can be calculated using custom designed circuitry. In this circuitry, multiplications by zero and one, and additions 
by zero are eliminated. This design is a complicated one, especially for a large block size $N$.

Referring to equation 21 of [24], each $G_{i}(n)$ has $i$ non zero elements at its $i+1^{\text {st }}$ row, plus a one at the diagonal. For example $G_{1}(n)$ has one non_zero element and a one in the second row. And $G_{2}(n)$ has two non zero elements and a one in its third row. Therefor in the product of first two matrices, $G_{2-1}(n)=G_{2}(n) G_{1}(n)$ only one multiplication of two real numbers and one addition of two real numbers are performed. Multiplying numbers by one and adding numbers by zero are overlooked. $G_{2-1}(n)$ is a lower triangular matrix with all ones on its diagonal. It also has one non zero element on the second row and two non_zero elements on the third row.

Now consider the product of $G_{3}(n)$ and $G_{2-1}(n)$ matrices, $G_{32_{2-1}}(n)=G_{3}(n) G_{2-1}(n)$. The first element of the third row of $G_{32-1}(n)$ requires multiplication of two pair of numbers and additions of three numbers. The second element of the third row of $G_{3-2-1}(n)$ requires multiplication of the two numbers and addition of the two numbers.

Continuing the same procedure $G(n)$ is calculated. The calculation of $G(n)$ needs a total of $Z$ multipliers and $Z$ adders where

$$
Z=\sum_{k=1}^{N-2}\left[\log _{2}(k)\right]
$$

Calculating and having these multiplications and additions ready, $G(n)$ can be calculated with a time delay of

$$
T_{3 G(n)}=(N-2) T_{m u l l}+(Z) T_{\text {add }}
$$

In equation $28 \mathrm{a}$, three separate calculations must be performed. First, calculate the second term in the bracket, filtering part. Then, subtract the result of the filtering part 
from the desired outputs, $y$ 's, to produce the sub_error vector. Finally multiply $G(n)$ to the sub_error vector to calculate the crror vector, e.

In the filtcring part, $2(N-1)$ combinations of of $H_{k}$ 's are calculated. Each $H_{k}$ is a row vector with length of $\frac{L}{N}$. It requires $2(N-1) \frac{L}{N}$ adders to produce these $2(N-1)$ combinations in a time delay of $(N-1) T_{\text {add. }}$ For $N$ combinations of $A_{k}$ 's, $N \frac{L}{N}$ adders are required in order to calculate all those combinations in one $T_{a d d} . \frac{2(N-1) N}{2}$ inner vector products of $A H$ with length of $\frac{L}{N}$ requires $N(N-1) \frac{L}{N}$ multipliers and adders. It completes the inner products in a time delay of $T_{\text {mult }}+\left\lceil\log _{2}\left(\frac{L}{N}\right)\right\rceil T_{\text {add. }}$. The last stage of the filtering is to add all scalar values in each row to obtain the filtering vector. Each row of filtering vector, such as one that is illustrated in equation 30 of the [24], has at least $N$ elements. Thus, it needs $N^{2}$ adders for a parallel implementation of all the $N$ rows, in a time delay equal to $\left[\log _{2} N\right\rceil T_{\text {add. }}$.

The sub_error vector, with a length of $N$, is the result of the subtraction of the filtering vector from desired output vector. It needs $N$ adders to execute it in one $T_{\text {add }}$ time delay.

The last part of equation $28 \mathrm{a}$ is calculating the product of $G(n)$ and sub_error vector. $G(n)$ is a $N \times N$ lower triangular matrix with $\frac{N(N-1)}{2}$ non zero and non_diagonal elements. All the diagonal elements of $G(n)$ are one. The product of a matrix and a vector can be obtained using the general systolic array suggested in [28]. In this way it requires $N$ PE's and $3 N\left(T_{\text {mult }}+T_{\text {add }}\right)$ time. The multiplication of $G(n)$ matrix and sub_error vector can be performed using $N-1$ multipliers and adders with a time delay of $(N-1)\left(T_{m u l t}+T_{\text {add }}\right)$. Also this production can be executed with $\frac{N(N-1)}{2}$ multipliers and adders with a time delay of $T_{\text {mult }}+\left[\log _{2}(N)\right] T_{\text {add }}$. 
Execution of equation $28 \mathrm{~b}$ has three stages. First, calculate all $N$ combinations of the errors. The longest combination includes $N$ elements. This task needs $N$ adders to produce all the combinations in $\left\lceil\log _{2}(N)\right\rceil T_{\text {add }}$ time delay. Second, multiply $N$ combinations of crrors to $N$ combinations of $A$ 's. Assume there are $\frac{N^{2}}{2}$ combinations of $A$. It cither can use $\frac{N^{3}}{2}$ multipliers to perform this task in one $T_{\text {mult }}$ delay time, or use $N$ multipliers to complete calculation in a $\frac{N^{2}}{2} T_{m u l t}$ delay time. Now cach vector of $H_{k}$ of equation $28 \mathrm{~b}$ is correspond to $N$ vectors of the right hand side of equation $28 \mathrm{~b}$, as exemplified in equation 32 . For these $2 N$ additions of the vectors of length $\frac{L}{N}$ either one can use $\frac{L}{N}$ adders and complete the calculation of the right hand side of equation $28 \mathrm{~b}$ in $2 N T_{\text {add }}$ time delay, or uses $2 L$ adders with $\left\lceil\log _{2}(N)\right\rceil T_{\text {add }}$ time delay. The last part of equation $28 \mathrm{~b}$ needs $L$ adders and $T_{\text {add }}$ delay time to produce the new tap weights of the adaptive filter. 


\section{BIBLIOGRAPHY}

[1] S. Haykin, "Adaptive Filter Theory," Englewood Clifton, NJ: Prentice-Hall, 1986.

[2] S.T. Alexander, "Adaptive Signal Processing," New York, Springer-Verlag, 1986.

[3] L. H. Sibol, "Adaptive Signal Processing," IEEE Press, 1987.

[4] P. Denyer and D. Renshow, "VLSI Signal Processing: A Bit-Serial Approach," Addison-Weslcy, 1985.

[5] M. Ishikawa, T. Tsukahara, and T. Kimura, "VLSI Architecture for An Adaptive Equalizer," Proc. Inter, Conf. on Acoust., Speech, Signal Processing, Tokyo, Japan, 1986.

[6] K. H. Cheng, and S. Sahni, “A New VLSI System for Adaptive Recursive Filtering," Parallel Computing, vol. 10, no. 1,March 1989.

[7] M. Yaminysharif, and T.S. Durrani, "Adaptive Signal Processing Using a Modified Gradient Estimation Technique," Proc. Inter. Conf. on Acoust, Speech, Signal Processing, Tokyo, Japan, 1986.

[8] G. Carayannis, D. G. Manolakis, and N. Kalouptsidis, "A Fast Sequential Algorithm for Least- Squares Filtering and Prediction," IEEE Trans. Acoust., Speech, Signal Processing, vol. ASSP-31, no. 6, Dec. 1983.

[9] S. Karni and G. Zeng, "A New Convergence Factor for Adaptive Filters," IEEE Trans. on Circuits and Systems, Vol. 36, No. 7, July 1989.

[10] D. T. M. Slock, "On the Convergence Behavior of the LMS and the Normalized LMS Algorithms," IEEE Trans. on Signal Processing, Vol. 41, No. 9, September 1993.

[11] C. F. N. Cowan, S. G. Smith, and J. H. Elliott, "A Digital Adaptive Filter Using a Memory-Accumulator Architecture: Theory and Realization," IEEE Trans. on Acoustics, Speech, and Signal Processing, Vol. ASSP-31, No. 3, June 1983.

[12] K. H. Cheng and S. Sahni, "A New VLSI System for Adaptive Recursive Filtering," Parallel Computing, Vol. 10, pp. 109-115, 1989.

[13] G. A. Clark, S. K. Mitra, and S. R. Parker, "Block Implementation of Adaptive Digital Filters," IEEE Trans. Acoust., Speech, Signal Processing, vol. ASSP-29, no. 3, June 1981. 
[14] K. Kurosawa, and S. Tsujii, "A New Type Adaptive Algorithm of Parallel Type Structure," Proc. Inter, Conf. on Acoust., Speech, Signal Processing, Tokyo, Japan, 1986.

[15] K. K. Parhi, and D. G. Messerschmitt, "Concurrent Cellular VSLI Adaptive Filter Architecture," IEEE Trans. Circuits Syst. Oct. 1987.

[16] K. Takahashi, "Performance improvement of LMS algorithm," Electronics and Communication in Japan, Part 3, Vol. 75, No. 5, 1991.

[17] J. A. Starzyk, and M. Eshghi, "Highly Parallel Adaptive Filter," Proc. Inter. Symp. on Circuits and Systems, Portiand, Oregon, May 1989.

[18] M. Eshghi, "Highly Parallel Transversal Adaptive Filters," M.S. Thesis, Ohio University, March 1988.

[19] N. Kobayashi, H. Gambe, K. Aoki, M. Koshikawa, and T. Ikezawa, "An Adaptive Transversal Filter VLSI," Proc. Inter. Conf. on Acoust., Speech, Signal Processing, Tokyo, Japan, 1986.

[20] B. A. Cochran and J. A. Cadzow, "Linear Recursive Adaptive Equalization Utilizing a Modified Gauss-Newton Algorithm," IEEE Trans. on Circuits and Systems, Vol. CAS-34, No. 7, July 1987.

[21] K. Hashimoto and H. Kimura, "A Parallel Archilecture for Recursive Least Square Method," Electronics and Communications in Japan, Part 1, Vol. 70, No. 4, 1987.

[22] D. Mansour, "A Highly Paraliel Architecture for Adaptive Multichannel Algorithms," Proc. of Inter. Conf. on Acoust, Speech, and Signal Processing, Tokyo, Japan, 1989

[23] S. E. McQuillan, and J. V. McCanny, "A VLSI Architecture for Multiplication, Division and Square Root," Inter. Conf. on Acoustics, Speech, and Signal Processing, Toronto, Canada, May 1991.

[24] J. Benesty, and P. Duhaamel, "A Fast Exact Least Mean Square Adaptive Algorithm". IEEE Transaction on Signal Processing, vol. 40, No. 12, December 1992.

[25] S. Park, "A VLSI Architecture For Adaptive Signal Processing," IEEE Proceedings of the Southeastcon, 1991.

[26] R. Simar Jr., "The TMS320C40: A DSP for Parallel Processing," Inter. Conf, on Acoustics, Speech, and Signal Processing, Toronto, Canada, May 1991.

[27] D. E. Borth, I. A. Grson, J. R. Haug, C. D. Thompson, "Flexible Adaptive FIR filter VLSI IC", IEEE Journal on Selected Areas in Communications, April 1988.

[28] C. Mead and L. Conway, "Introduction to VLSI Systems," Addison-Wesley, 1980.

[29] B. Widrow and J. M. McCool, "A comparison of adaptive algorithms based on the method of steepest descent and random search," IEEE Trans. Antennas Propagat., vol. AP-24, pp. 616-637, Sept. 1976. 